\title{
Migrationsstress, Alter und Lernen - Betrachtungen der Zusammenhänge sowie Implikationen für die Didaktik \\ (am Beispiel in Deutschland lebender, \\ gealterter türkischstämmiger Menschen)
}

Dissertation

zur Erlangung des sozialwissenschaftlichen Doktorgrades der Sozialwissenschaftlichen Fakultät der Georg-August-Universität Göttingen

vorgelegt von

Ibrahim Özkan

aus Babadağ / Türkei

Göttingen 2011 
1. Gutachter

$\underline{\text { Prof. Dr. Hans- Dieter Haller }}$

2. Gutachter

Prof. Dr. Ulrich Sachsse

Tag der mündlichen Prüfung

$\underline{08.07 .2011}$ 


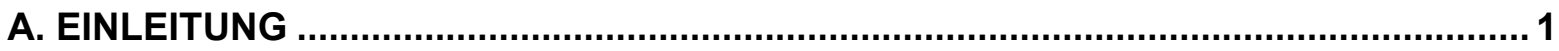

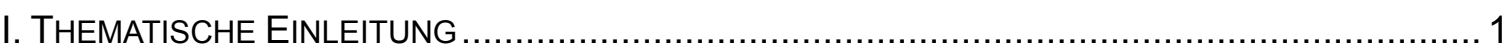

II. ERKENNTNISINTERESSE, ZIELSETZUNG UND VORGEHEN ......................................... 10

1. Erkenntnisinteresse und Zielsetzung: Durch Methodik kann in Deutschland gealterten Migranten zu einem „Wissens-“erwerb (ver-) helfen? .............................. 10

2. Vorgehen auf Basis der Grounded Theory ....................................................... 11

\section{B. BETRACHTUNG DER ZUSAMMENHÄNGE ZWISCHEN MIGRATIONSSTRESS,}

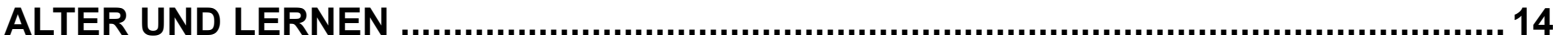

I. Bisheriger Forschungsstand aus den Bereichen MigRation, Alter, LERNEN UND

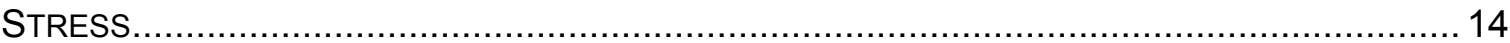

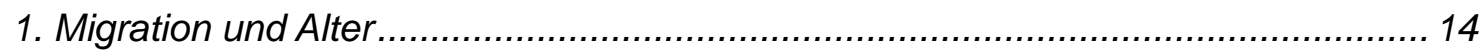

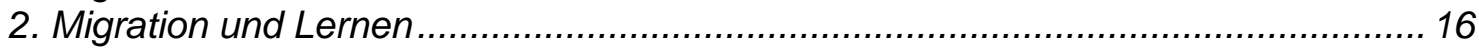

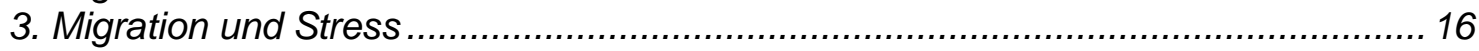

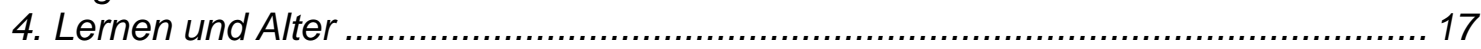

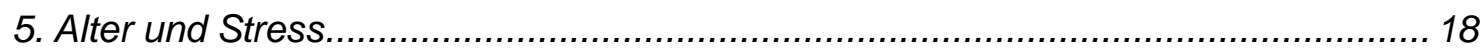

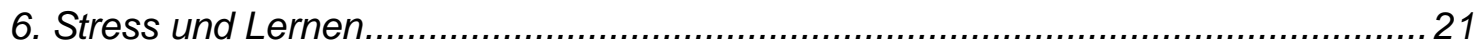

II. BETRACHTUNG DER ElEMENTE: MIGRATION - ALTER - LERNEN UND STRESS .................. 22

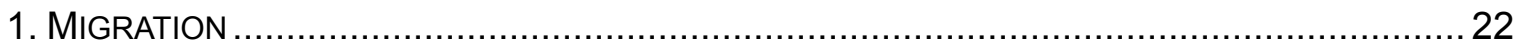

$1.1 \mathrm{Am}$ Anfang steht die Kultur - was ist das? ................................................. 22

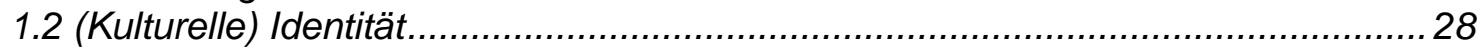

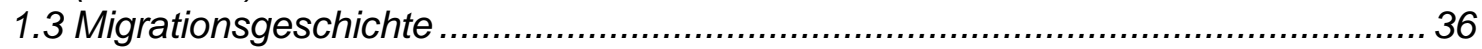

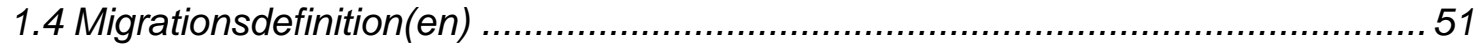

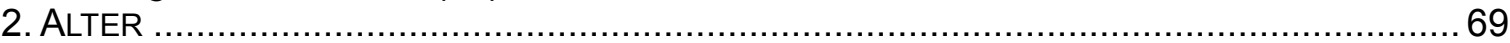

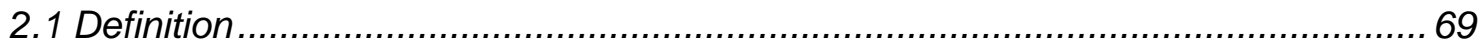

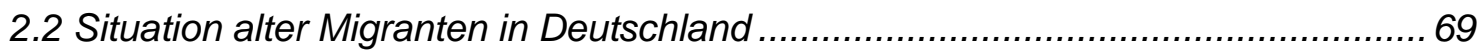

2.3 Perspektive der alten Migranten in Deutschland ........................................... 74

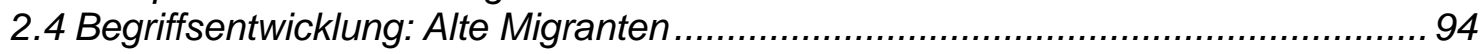

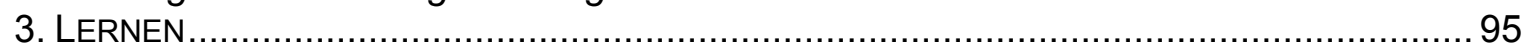

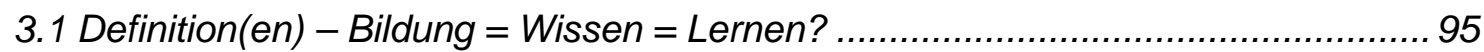

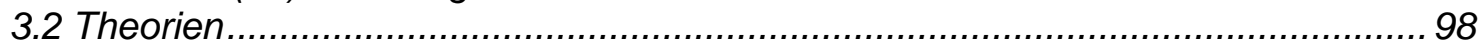

3.2.1 Lebenslanges Lernen und Lernen im Alter ............................................. 104

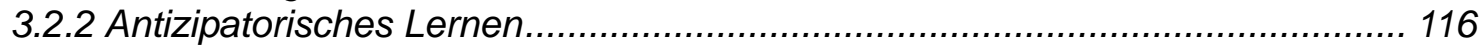

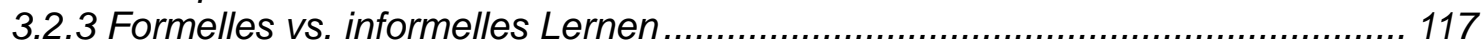

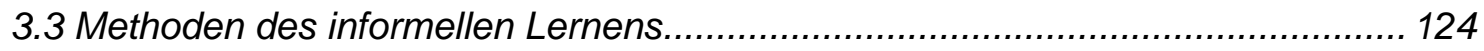

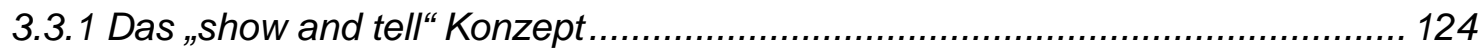

3.3.2 Exkurs: Was ist ein (sprachliches) Zeichen und wozu?.............................. 126

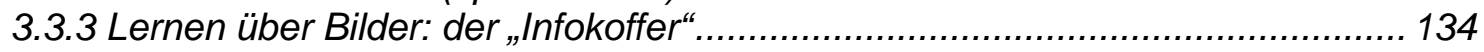

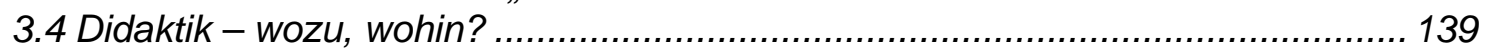

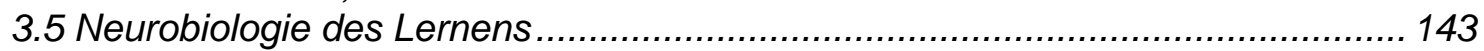

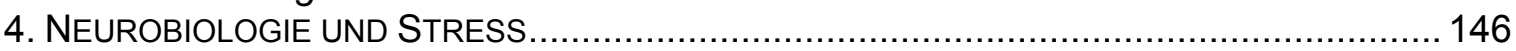

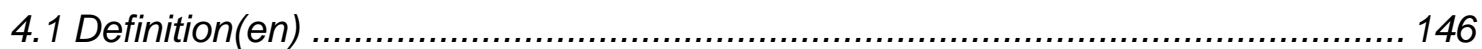

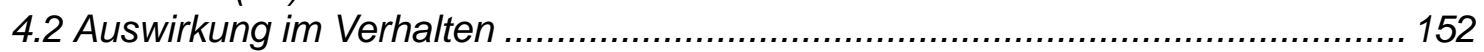

4.3 Soziale Auswirkungen von Stress (Gruppen- und „Herden“-Verhalten)................ 156

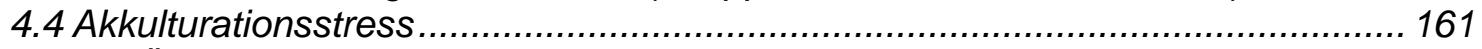

III. FINALE ÜBERLEGUNGEN UND BELEGE ........................................................... 174

1. Akkulturationsstress als mögliche Lernblockade ............................................. 174

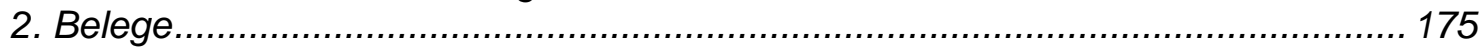

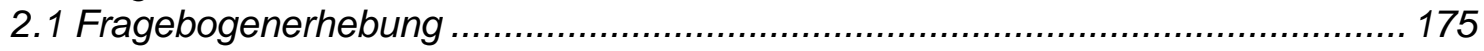

2.2 Falldarstellungen aus der psychotherapeutischen Praxis............................... 181

2.3 Übersicht über Abbruchstatistik von Integrationskursen .................................. 190 


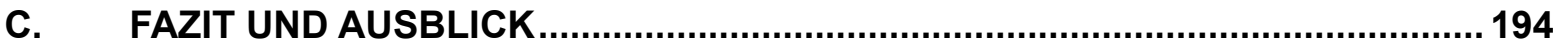

1. ZUSAMMENFASSUNG UND ABSCHLIEßENDE ÜBERLEGUNGEN ..................................... 194

2. 'INFOKOFFER' ALS MÖGLICHES DIDAKTISCHES WERKZEUG ........................................20

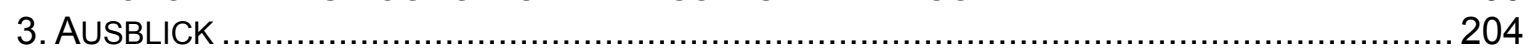

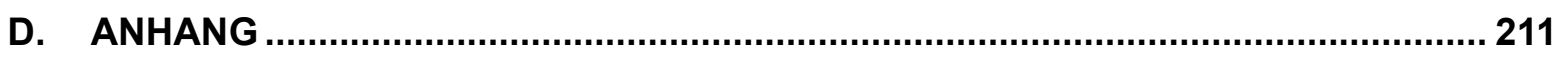

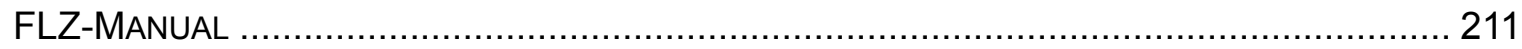

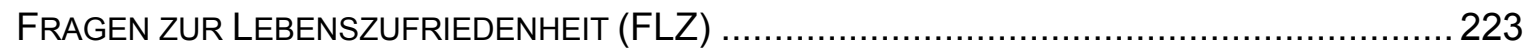

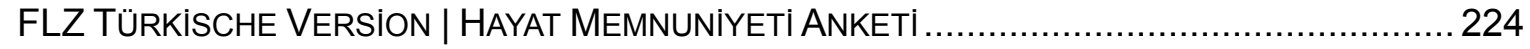

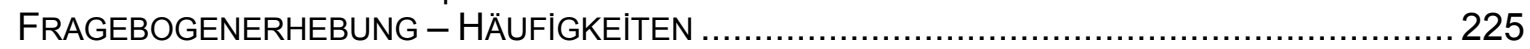

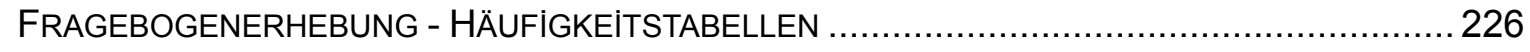

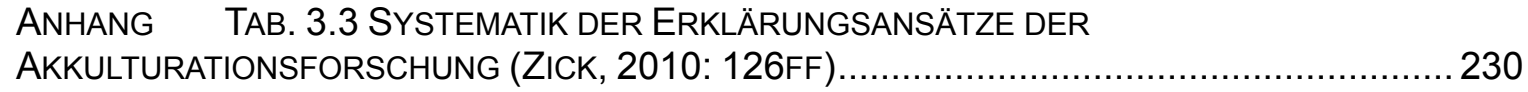

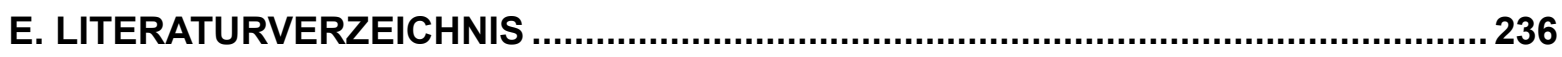


„Was ich nicht erlernt habe, das habe ich erwandert." Johann Wolfgang von Goethe

\section{A. Einleitung}

\section{Thematische Einleitung}

Bereits im 18. Jahrhundert hat Johann Wolfgang von Goethe mit nur einem Satz formuliert, was Gegenstand dieser Arbeit ist. Jenes, was Goethe (formal) nicht erlernt hat, hat er auf andere Art und Weise (informell) gelernt. Sein Weg war die Wanderung ${ }^{1}$. Da der Weg bei jedem Individuum jedoch unterschiedlich gestaltet sein kann, bedarf es verschiedenster Angebote und Konzepte, um einer jeden Lerner-Persönlichkeit zu entsprechen. „Je nach der individuellen Geschichte wird Migration für den Einzelnen zum 'Traum' oder zum 'Trauma'“' (SALMAN, 1995). Die Zielgruppe der vorliegenden Arbeit sind primär türkischstämmige, gealterte Migranten - ehemalige Wanderer sozusagen (Migration vom lat. migrare wandern; auswandern). Dabei handelt es sich um eine, trotz oftmals hohen Alters, junge Zielgruppe - in dem Sinne, dass das Thema der gealterten Migranten stetig an Aktualität gewinnt und 'neu' entdeckt werden musste. Man könnte sogar sagen, mit jedem Tag, den die Bundesrepublik Deutschland nach dem Zweiten Weltkrieg altert, altert auch die angeführte Zielgruppe. Zumal sie als solche in Deutschland erst seit dem 'Wiederaufbau' durch das Anwerbeabkommen der damals so genannten Gastarbeiter auftreten konnte, obgleich Migration ein weitaus älteres, globales Phänomen ist.

Eng verknüpft mit dem Begriff der Migration ist selbstverständlich auch die Integrationsfrage. Diese Frage gewinnt in den letzten Jahren zunehmend an politischer und auch sozio-kultureller Bedeutung - selbst der ehemalige Bundeskanzler Gerhard Schröder brachte das Thema Einwanderung in seiner Regierungserklärung vom 10.11.1998 zur Sprache und betonte, die einstigen Gäste seien inzwischen längst Mitbürgerinnen und Mitbürger und von daher keine Fremden mehr. Der Bedeutungsgewinn zeigt sich beispielsweise auch darin, dass sich sprachliche Begrifflichkeiten ausdehnen. So ist heute 'politisch korrekt' nicht mehr von 'Ausländern' die Rede, sondern vielmehr von 'Menschen mit Zuwanderungsgeschichte'. Diese Formulierungsveränderung setzt die Geschichte

$\mathrm{Zu}$ dieser Zeit war es im Adel noch üblich, die Jugend zu so genannten Bildungsreisen zu veranlassen. Ein Blick in Goethes Autobiografien jedoch zeigt, dass er lebenslang auf Bildungsreisen war und sich während und durch das Reisen gebildet hat. 
dieser Menschen durch ihre explizite Erwähnung mit in den Fokus. Dieser Fokus hat psychologisch, soziologisch, neurologisch, kulturanthropolgisch, didaktisch, ökonomisch und natürlich unter anderem politisch ${ }^{2}$ eine klare Funktion und Daseinsberechtigung. Der Sozialwissenschaftler Wolf-Dietrich BuKOW bezeichnet ein Phänomen aus diesem Kontext als „sinnfremden Reduktionismus“. Mit diesem Begriff beschreibt er eine „alltagsrassistische“ Praxis, in welcher bestimmte Menschen über einen Sammelbegriff wie „Ausländer“, „Asylant“ oder auch „Migrant“ einer Gruppe zugeordnet werden und dieser Gruppe wiederum eine soziale Komponente unterstellt wird. Des Weiteren wird den über diese Begriffe definierten sozialen Gruppen unterstellt, spezifische Eigenschaften aufzuweisen (vgl. Bukow 2010: 38). Dieser Reduktionismus, in welchem „[...] oftmals alle irgendwie namhaft machbaren Probleme, Risiken, Verwerfungen, angefangen bei geringeren Bildungschancen und endend bei Gewalt in der Familie, absolut einseitig auf einen allochtonen Hintergrund reduziert werden“ (BUKOW 2010: 38), bleibt im unreflektierten Alltagsverständnis meist unentdeckt. Ähnliches führt Bukow hinsichtlich des Begriffs der „Parallelgesellschaft“ zu Felde, denn auch hier wird ein sinnfremder Reduktionismus praktiziert, in welchem unterstellt wird, „[...] dass Menschen, wenn sie ein Milieu ausbilden, das keine westlichen sondern östliche Kulturen zitiert, sich automatisch abschotten und ein Eigenleben entwickeln." (BUKOW 2010: 39) Dass in Bezug auf diese postulierten Parallelgesellschaften des Öfteren noch eine fundamentalistische Prägung attestiert wird, und somit der Fundamentalismus auch als ein allochtones Element dargestellt wird, stellt für BuKOW, ähnlich wie das oben aufgeführte Beispiel, einen Reduktionismus dar, welcher unentdeckt bleibt: „Er arbeitet ja zumeist mit Verweisen auf den Islam. Und dem traut man ja ohnehin seit alters her alles zu.“ (Bukow 2010: 39).

Verantwortlich dafür ist nach Bukow der Umstand, dass ein gewisser geistiger Aufwand dazu nötig ist, die durch den Alltagsrassismus erzeugten sozialen, kulturellen und religiösen Repräsentationen zu dekonstruieren, was sich angesichts der tagtäglichen Bestärkung dieser durch den öffentlichen Diskurs als zusätzlich erschwert darstellt. Hinzu kommt an dieser Stelle, dass dieser sinnfremde Reduktionismus, sofern er an „[...] den offenbar bis heute nachhaltig

2 Diese Aufzählung deutet bereits an, was sich im Lauf der Arbeit zeigen wird - die Thematik des Gesamtkomplexes 'Migration' ist ein Thema, dass interdisziplinär relevant ist und gesamtgesellschaftlich Bedeutung hat. 
wirksamen klandestinen Rassismus [...]“ (Bukow 2010: 40) anknüpft, zum Selbstläufer werden kann, obwohl objektiv gesehen Parallelgesellschaften in einer modernen, arbeitsteiligen Gesellschaft kaum mehr denkbar sind. Dennoch sind sie - die Repräsentationen - noch vorhanden, und betreffen beispielsweise die größte ethnische Minorität in Deutschland: die türkischstämmigen Menschen mit Zuwanderungsgeschichte.

Diese Geschichte, welche die persönliche Biografie eines jeden Einzelnen geprägt hat, ist gerade bei dieser Zielgruppe ein Einflussfaktor auf ihr Vermögen zu lernen und für ihre Lebenswelt im 'Nicht-Heimatland', das dennoch ihr Zuhause ist, prägend. Diesen und auch andere Einflüsse auf die Lern-Fähigkeit der gealterten Migranten wird die vorliegende Arbeit unter anderem näher beleuchten. Ebenfalls ein Schwerpunkt der Dissertation ist der angeführte Aspekt des Alters der Migranten, sowie die Migrationsprozesse (intra- und interpersonell) selbst, vor allem in Hinblick auf einen weiteren Kernaspekt: Migrationsstress.

Das Alter der Migranten steht im Fokus der Arbeit, da alternde, in der Türkei geborene Menschen in Deutschland eine besondere Gruppe darstellen. Vor allem sind im Alter Unterschiede in den Lebenswelten alter Menschen mit Zuwanderungsgeschichte festzustellen, verglichen mit gealterten Deutschen in Deutschland. Die Migranten beziehen beispielsweise weniger finanzielle Mittel, obwohl sie Ansprüche auf mehr hätten, laufen stärker Gefahr in Armut abzudriften, da sie z.B. über keine Grundsicherung oder soziale Stütze verfügen, oder diese nicht wahrnehmen:

Zentrale Bausteine für Wohlbefinden im Alter und den Erhalt der selbstständigen Lebensführung sind Wohnen, Gesundheitsstatus und Alterseinkommen. Die vorhandenen Daten zeigen, trotz bestehender Wissenslücken, für die Mehrheit der älteren Menschen mit Zuwanderungsgeschichte ein im Vergleich zur Altersbevölkerung ohne Migrationshintergrund durchschnittlich niedriges Alterseinkommen und ein deutlich höheres Armutsrisiko (Grundsicherungsquote im Alter: $13 \%$ ) mit erheblichen Disparitäten innerhalb der unterschiedlichen Gruppen. Allein lebende Frauen haben in allen Gruppen das höchste Armutsrisiko, allerdings leben allein stehende Migrantinnen weniger oft in EinPersonenhaushalten und öfter im Familienverbund. (DEUTSCHER VEREIN FÜR ÖFFENTLICHE UND PRIVATE FÜRSORGE E.V. - 2010: 3f)

Einige der Migranten können beispielsweise die Grundsicherung auch gar nicht wahrnehmen, da es ihnen an Informationshintergrund und Bildung mangelt. Andere empfinden sich nicht in der Position, finanzielle Unterstützung von einem Staat zu erfahren, in dem sie nicht durchgängig leb(t)en - da viele der türkischstämmigen Migranten über die Sommermonate in die türkische Heimat zu Verwandten reisen. Diffiziel ist zudem die sichere Erhebung der Hintergründe, die 
Menschen mit Zuwanderungsgeschichte dazu bringt, beispielsweise die ihnen zustehende Grundsicherung nicht wahrzunehmen ${ }^{3}$. Einer der Gründe für die mangelnde Annahme der Grundsicherungsangebote ist beispielsweise auch die Sprachbarriere, die den Migranten das Verständnis ihrer Optionen erschwert. Um dies zu überwinden, können z.B. qualifizierte Dolmetscher eingesetzt werden. Konzepte hierfür existieren bereits (Telefondolmetscher etc.) - vor allem ist jedoch ein kultursensibler Umgang erforderlich, der über die reine sprach-übermittelnde Kompetenz hinausgeht und auch kulturelle Kenntnisse impliziert. Beispielsweise ist ein Bewusstsein für die Bedeutung von Krankheiten in spezifischen Kulturen hilfreich:

Die kultursensible Übermittlung von Gesundheitsinformationen erfordert über die sprachliche Ebene hinausgehende Kompetenzen im Sinne einer Sprach- und Kulturvermittlung. Die hier eingesetzten Dolmetscherinnen sind für diese Aufgabe eigens qualifizierte sprachkundige Migrantinnen. Ihre Qualifikation umfasst: kultursensibles Wissen (zum Beispiel Davos, tradiert das Krankheitsverständnis), kenntnisnotwendige Fachbegriffe, Neutralität (kommentarlos übersetzen) und Verpflichtung zur Verschwiegenheit. Flächendeckend stehen solche Dolmetscherinnen nicht zur Verfügung und die verfügbaren Angebote sind unzureichend bekannt. Inzwischen ist auch ein telefonischer Dolmetscherservice "SprachXPress“ entstanden, der in kürzester Zeit (zwei Minuten) bundesweit eine/n Dolmetscher/in zur Verfügung stellen kann. Dieses Modell ist in mehreren europäischen Ländern alltagserprobt und wird regelhaft im Gesundheits- und Sozialdienst eingesetzt. (DEUTSCHER VEREIN FÜR ÖFFENTLICHE UND PRIVATE FÜRSORGE E.V. 2010: 15-16)

Diese Forderung wird bestärkt und noch plausibler, führt man sich den Terminus des Kulturrelativismus in diesem Kontext vor Augen:

Kulturrelativismus, umgangssprachlich: Jede Kultur hat eben ihre eigenen Traditionen und Bräuche. Es macht keinen Sinn darüber zu streiten.

Wissenschaftlich: Kulturen sind je spezifische Systeme der Daseinsvorsorge und sozialen Kontrolle und haben ihre Eigenlogik. [Hervorhebung i.O.] (AUERNHEIMER 2007: 138)

Weiterhin ist auch der Entwicklung der Gesellschaft dahingehend Sorge zu tragen, dass generelle Veränderungen notwendig sind, wie z.B. die interkulturelle Öffnung der Altenhilfe oder auch die Versorgung alter Menschen mit Zuwanderungsgeschichte. Dies ist nicht zuletzt darin begründet, dass die Gesellschaft in Deutschland generell altert und gleichzeitig die Zahl der zugewanderten Alten steigt. So konstatiert auch der DEUTSCHE VEREIN FÜR ÖFFENTLICHE UND PRIVATE FÜRSORGE E.V.:

[V]orrangig ist die interkulturelle Öffnung der Regelversorgung anzustreben. Interkulturelle Öffnung bedeutet die gezielte Verbesserung der Bedingungen unter denen Rechte verwirklicht werden können.

Für mehr Gesundheitschancen sollen Programme zur Aufklärung, Gesundheitsförderung, Prävention, Behandlung und Rehabilitation von Sozialversicherungsträgern sowie Bund,

3 Vgl. Deutscher Verein für öffentliche und private Fürsorge e.V. - 2010: 3-4; Fußnote 7. 
Ländern und Kommunen spezifische, kultursensible Handlungsansätze für ältere Migrantinnen und Migranten enthalten, die unterschiedliche Bildungsniveaus berücksichtigen. Zum Abbau bestehender Zugangs- und Nutzungsbarrieren und gesundheitlicher Ungleichheit sind vorhandenes Wissen und praxiserprobte Handlungsansätze, wie sie z.B. das Ethno-Medizinische Zentrum in Hannover mit dem Projekt "Mit Migranten für Migranten“ oder die Stadt Hannover mit der Qualifizierung von „Integrationslotsen“ erarbeitet hat, in die Fläche zu bringen.

Die wachsende Zahl älterer Migrant/innen und ihre früher einsetzende Alterung und Pflegebedürftigkeit erfordert mehr Anstrengungen zur interkulturellen Öffnung der Altenhilfe in den Kommunen. Kultursensible, mehrsprachige, leicht verständliche Informationen zu Gesundheitsfragen im Alter (Lebensstil, Demenz usw.), Gesundheitswegweiser zu mehrsprachigen Gesundheitseinrichtungen und Informationen zu Strukturen und Angeboten der Altenhilfe und Pflege (offene, ambulante Dienste, Wohnformen) sollten vor Ort vorhanden sein. Zielgruppen zur Verbreitung sind nicht nur die älteren Menschen selbst, sondern auch Vertrauenspersonen wie Vorstände von Gemeinden, Missionen, Vereinen usw. Sinnvoll sind Mehrgenerationenansätze, so können über jüngere Angehörige z.B. in Integrationskursen auch die Älteren erreicht werden. (2010: 16-17)

Forderungen wie diese bilden einen der basalen Grundpfeiler des Ziels dieser Arbeit: eine Idee vorzustellen und die Hintergründe - auch neurobiologisch zu erörtern, die der gesellschaftlichen Entwicklung hinsichtlich der zunehmenden Zahl an alten Migranten in Deutschland Rechnung tragen können, und möglicherweise einen Ansatzpunkt für weitere Forschung in diesem Bereich bieten.

Ein Anliegen, das im Zentrum dieser Arbeit steht, ist, durch kultursensible Ansätze Prävention im Bereich der psychischen Störungen zu leisten. Die Serie 'Migrationspsychiatrie' beleuchtete die Notwendigkeit für die Zielgruppe der türkischen Gemeinschaft in Deutschland beispielsweise 2008 in einem Artikel, der eben diese Problematik bereits im Titel enthält: 'Kultur und Ethnizität - Ihre Rolle bei der Entstehung psychischer Störungen am Beispiel der türkischen Gemeinschaft in Deutschland'. Auch hierin werden mangelnde Sprachkenntnisse und Unwissenheit über das deutsche Gesundheitswesen als Gründe genannt, warum eine verminderte Inanspruchnahme der Möglichkeiten besteht. Diese führt letztlich jedoch zu psychischen Störungen, welche wiederum hohe und unnötige Kosten verursachen, da Präventionen möglich sind. (vgl. GÜL, ÖNER, UYAR 2008).

Das oben gesagte betrifft natürlich nicht nur die türkischstämmigen Migranten, sie bilden jedoch die größte Minorität, wie nachstehende Tabelle „In Deutschland lebende Hauptgruppen von Ausländern nach Alter“ zeigt: 
Tabelle 1: In Deutschland lebende Hauptgruppen von Ausländern nach Altersgruppen

\begin{tabular}{|l|r|r|r|r|}
\cline { 2 - 5 } \multicolumn{1}{c|}{} & \multicolumn{1}{c|}{ Türkisch } & Italienisch & Griechenland & Spanien \\
\hline Alter & & & & \\
\hline$<5$ & 13.135 & 4.395 & 2.146 & 991 \\
\hline $5-10$ & 27.044 & 9.758 & 4.803 & 1.327 \\
\hline $10-15$ & 127.295 & 23.450 & 12.904 & 2.323 \\
\hline $15-20$ & 146.970 & 27.866 & 15.294 & 2.866 \\
\hline $20-25$ & 134.543 & 35.423 & 15.795 & 6.361 \\
\hline $25-35$ & 303.752 & 84.313 & 43.356 & 20.610 \\
\hline $35-45$ & 339.447 & 10.3644 & 57.822 & 24.777 \\
\hline $45-55$ & 206.128 & 94.827 & 46.211 & 16.941 \\
\hline $55-65$ & 149.786 & 76.020 & 37.078 & 13.237 \\
\hline $65-75$ & 147.489 & 40.457 & 28.966 & 9.939 \\
\hline $75-85$ & 32.112 & 14.791 & 11.330 & 5.316 \\
\hline $85-95$ & 1.669 & 2.382 & 901 & 675 \\
\hline$>95$ & 110 & 220 & 79 & 38 \\
\hline Gesamt & 1.629 .480 & 517.546 & 276.685 & 105.401 \\
\hline
\end{tabular}

Quelle: Statistisches Bundesamt ${ }^{4}$

Im Verhältnis zu einer Gesamtbevölkerung, die sich in einem demografischen Wandel befindet und sich gegenwärtig reduziert, leben auffällig viele Menschen mit Zuwanderungsgeschichte in Deutschland, welche zudem einen deutschen Pass besitzen, jedoch daher in obiger Tabelle nicht erfasst sind.

Doch wer sind diese Menschen? Der durchschnittliche 'Migrant' in Deutschland ist 36,7 Jahre alt, stammt meist aus der Türkei und ist geprägt durch die Kultur der 'Alten'. Laut Definition der BUNDESREGIERUNG von 2001 umfasst „Migration [...] sowohl Zu- als auch Abwanderung und steht für die räumliche Bewegung zur Veränderung des Lebensmittelpunktes von Individuen oder Gruppen über eine sozial bedeutsame Entfernung“ (BUNDESREGIERUNG 2001: 3). Hierunter fallen auch die im Dezember 2000 erfassten 1.998.534 Millionen Menschen türkischer Herkunft in Deutschland, davon 712.880 mit einer befristeten Aufenthaltserlaubnis, alle weiteren mit entweder einer unbefristeten Aufenthaltserlaubnis, einer Aufenthaltsbewilligung, -befugnis oder Duldung (BundesRegIeRUNG 2001: 80f). Damit bildeten sie Ende des Jahres 2001 hierzulande mit einem Anteil von 26,6 \% die größte Gruppe der ausländischen Wohnbevölkerung (DEUTSCHER BUNDESTAG Berlin 2002: 142). Auch innerhalb der

\footnotetext{
${ }^{4}$ http://www.destatis.de/jetspeed/portal/cms/Sites/destatis/Internet/DE/Content/Publikationen/Fachv eroeffentlichungen/Bevoelkerung/MigrationIntegration/AuslaendBevoelkerung2010200107004,p roperty=file.pdf, aufgerufen am 26.05.2011.
} 
Gesamtheit der aktiv versicherten Ausländer in der Gesetzlichen Rentenversicherung stellen somit die Türken mit 27,0 \% (Jahr 2001) das größte Bevölkerungskontingent (HACKHAUSEN 2001: 20). Insgesamt bezogen in der Bundesrepublik Deutschland bereits im Jahr $1995^{5} 30.780$ Türken eine Altersrente, 46.100 Türken eine Rente wegen verminderter Erwerbsfähigkeit sowie 26.500 Türken eine Witwen- bzw. Witwerrente. Abzüglich der 30.970 Rentenempfänger, die ihre Rente im Heimatland beanspruchten, ließen sich für dieses Jahr also 72.710 türkische Rentenempfänger in der Bundesrepublik ermitteln (SEEBERGER 2001: 30).

Konkrete Angaben zur gerontologischen Demographie legt SEEBERGER z.B. für die Stadt Nürnberg im Jahr 1999 vor: Hier lebten insgesamt 83.303 nichtdeutsche Bewohner, 1.520 Personen davon waren zwischen 65 und 70 Jahre alt, 7.128 Personen zwischen 55 und 65 Jahren. Innerhalb der türkischen Volksgruppe stellen die mittlerweile alt gewordenen Türken der ersten Generation eine Minderheit dar (SEeberger 2001: 4f). In Frankfurt wiederum betrug laut dem Deutschen Roten KREUz (DRK) im Jahr 1998 der Migrantenanteil (inklusive der Aussiedler) gut $30 \%$, worunter sich der Anteil ausländischer Senioren über 60 Jahre mit 11,5\% im Vergleich zu den 22\% über 60-jährigen deutschen Stadtbewohnern vergleichsweise gering ausnahm. Allerdings sei mit einem raschen und kontinuierlichen Anstieg der alten ausländischen Bewohner zu rechnen, da bei den 50-59-jährigen Frankfurtern bereits jeder Vierte zu dieser Bevölkerungsgruppe gehörte (DRK 2002: 10). Nicht zuletzt inspiriert durch das diesjährige 50-jährige Jubiläum des Anwerbeabkommens lohnt es sich also, diese spezielle Gruppe und ihre Lebenswelt zu betrachten.

Neben dem sich verändernden Alter und den sich wandelnden Begrifflichkeiten (vom 'Ausländer' zum 'ausländischen Mitbürger' und schließlich zu 'Menschen mit Migrations-/Zuwanderungshintergrund') verändert sich jedoch auch intrapersonell etwas. Die innere Haltung und das Selbstverständnis der jahrelang als 'Ausländer' bezeichneten Menschen ist durch diese Begrifflichkeit selbstverständlich nicht unwesentlich geprägt. Auch 'türkische Mitbürger' impliziert genau wie 'Gastarbeiter' als Bezeichnung eine Identifizierung als 'Nicht-Deutsche', im weitesten Sinne oftmals nach wie vor als 'Fremde' und zieht damit

\footnotetext{
${ }^{5}$ Bedauerlicherweise läßt der DEUTSCHE BUNDESTAG in seinem Bericht von 2001 über die Lage der Ausländer in der BRD jegliche aktuelle Auskunft vermissen (vgl. DT. BUNDESTAG 2001).
} 
gesamtgesellschaftlich $^{6}$ eine Grenze, an welcher sich 'integrationswillige' Menschen stetig bewegen: zwischen der tradierten Kultur des Heimatlandes und der gelebten Kulturform des Aufenthaltslandes. Führt man sich zusätzlich vor Augen, dass einige ehemalige Gastarbeiter ihren Lebensabend, bevor sie nach Deutschland kamen oder auch während sie hier arbeiteten, in ihrem Heimatland imaginiert hatten, aufgrund ihrer Lebensumstände jedoch nicht zurückkehren konnten oder wollten, liegt die Metapher der 'Grenzgänger' nahe. Es tritt eine (unbewusste/unterbewusste) Kollision von Erwartungen, Hoffnungen und der Realität ihrer Lebenswelt auf, die zu Stress führen kann. Entgegen der statistischen Durchschnittszahlen finden sich problemfrei ältere, ehemalige Gastarbeiter, die schlicht nicht auf das Altern in Deutschland vorbereitet waren und mit der Versorgung überfordert sind. Dies liegt unter anderem an dem Bildungsgrad, mit welchem sie als Gastarbeiter nach Deutschland kamen. Der Bildungsgrad und die Lebensumstände erlaubten innen jedoch auch keine Bildungserweiterung und -vertiefung, da sie lediglich zum Arbeiten und Wiederaufbau des Landes angeworben worden waren. Sie sind daher an die neu existierenden Versorgungsstrukturen nicht angepasst und aufgrund von u.a. der Sprachbarriere fällt innen der Informationserwerb darüber zudem schwer. Neben diesen Faktoren addiert sich ein Aspekt, der unter anderem in der Psychologie, Soziologie und Kulturanthropologie verortet ist: Identitätsaufrechterhaltung. Um Identität aufrecht zu erhalten ist es nötig, zu wissenwer man ist - traditionell ist dafür als erste Reflexionsfläche die Familie oder eigene Kultur die Anlaufstelle. Damit dienen Familie und Kultur zwar als Sicherheit für die Individuen, aber gleichermaßen besteht die Gefahr des Rückzugs aus der Gesellschaft. Damit verbunden fällt der Informationserwerb zu Versorgungsstrukturen schwerer. Auch diese Faktoren können somit zu nicht unerheblichem Stress führen.

Der Ursprung für diese Art von Stress liegt basal in der Tatsache begründet, dass die ehemaligen Gastarbeiter ihr Heimatland und damit ihre ursprüngliche Kultur verlassen haben, und somit die gegenwärtige Situation erst entstehen

6 BuKow postuliert, dass sowohl der öffentliche Diskurs, als auch der wissenschaftliche Diskurs einem Kulturprogramm bzw. nationalen Erzählungen folgen. Er sieht dies darin belegt, dass die Disskussionsstränge des öffentlichen Diskurses im Wesentlichen parallel verlaufen. Die Wirkung dieses Kulturprogramms beschreibt BuKow als „[...] ein fest verankertes, unverwüstliches, kritikresistentes, mit der Aufteilung der Wirklichkeit in "Wir und die Anderen“ arbeitendes Wirklichkeitsmodell“ (BuKOW 2010: 37). Dieses Wirklichkeitsmodell zielt auf „[...] die Durchsetzung einer bestimmten Konstruktion von Wirklichkeit - einer Wirklichkeit allerdings, die wie alle solche Programme darunter leidet, dass sie so niemals Realität war." (BukOW 2010:38) 
konnte. Wären sie in ihrem Land geblieben, hätten sie vermutlich mit anderen Stressoren umgehen müssen, Migrationsstress ist aber durch die Zuwanderung ein (meist) unbewusstes Thema im Leben vieler Menschen (mit eigener Zuwanderungsgeschichte ${ }^{7}$ ) und ist somit lohnenswert, näher betrachtet zu werden.

Da viele ehemalige Gastarbeiter aufgrund der veränderten Situation im Ursprungsland (beispielsweise des immensen Verstädterungsprozesses) oder auch ihrer aktuellen Lebenssituation nicht mehr zurückkehren können oder wollen, bleiben sie in Deutschland. Um jedoch nicht ausgewiesen zu werden, müssen sie u.a. die deutsche Sprache lernen, einen Einbürgerungstest bestehen oder Integrationskurse besuchen. Diese Auflagen führen zwangsläufig zu einer Art von Druck.

Die emotionale Vulnerabilität ist genetisch, neurobiologisch und biographisch bedingt. Es gibt empirisch gesicherte Daten, die Hinweis auf die emotionale Dysregulation geben. [...] So haben limbische; paralimbische und neokortikale frontale Strukturen Bedeutung für emotionale, motivationale, kognitive und motorische Verarbeitungsprozesse. (SENDERA und SENDERA 2005: 70-71).

Somit kann Migrationsstress als emotionale Vulnerabilität gegeben sein, insbesondere bei einem möglichen invalidierenden Umfeld. Insbesondere der Aspekt der biografischen Bedingtheit kommt in diesem Kontext zum Tragen. Addiert mit obig angeführtem Stress ist offensichtlich, dass diese Menschen unter enormer psychischer Belastung leiden (können) und es drängt sich die Frage nach Möglichkeiten der Stressreduktion und damit der Erhöhung der Lebensqualität ${ }^{8}$ auf. Für diese Frage bietet die vorliegende Arbeit Anreize und im Fazit einen Vorschlag für ein didaktisches Modell, mit dem man beispielsweise das Lernen stressfreier gestalten und damit eine höhere Erfolgsquote erzielen werden könnte.

7 Die Nachkommen der ehemaligen Gastarbeiter haben auch mit der Zuwanderungsgeschichte der Eltern oder zumindest mit der Tatsache dass sie zwischen den Kulturen gefangen sein können, zu tun. Gleiches gilt natürlich für die bundesdeutsche Bevölkerung, wie man an der Integrationsdebatte der letzten Jahre nachvollziehen kann. Eingedenk der Tatsache, dass die Zahl der Migranten in den westeuropäischen Ländern auf 5-10 Millionen Menschen geschätzt wird (BRANDRUP-LUKANOW 2000: 13), darunter im Jahr 2000 7,319 Millionen Ausländerinnen und Ausländer in der Bundesrepublik Deutschland (DEUTSCHER BUNDESTAG 2001: 142), wird inzwischen auch im amtlichen Sprachgebrauch der Tatsache Rechnung getragen, dass die Bundesrepublik ein „Zuwanderungsland“ ist.

8 Eine Erhöhung der Lebensqualität fordert ebenfalls die Weltgesundheitsorganisation (WHO), indem sie eine Prämisse formulierte nach Abbau sozialer und wirtschaftlicher Ungleichheiten zur Minderung daraus resultierender gesundheitlicher Folgen, also „öffentliche, private und freiwillige Ressourcen zur Verfügung [zu stellen], um den sozialen und gesundheitlichen Erfordernissen der am meisten gefährdeten Gruppen zu entsprechen und um allen, die dies benötigen, Zugang zu bedarfsgerechter, akzeptabler und nachhaltiger Versorgung zu verschaffen“ (BRANDRUP-LUNKANOW 2000: 15). 
Dieses 'Handwerkszeug' geht zurück auf die ursprüngliche Thematik dieser Dissertation, die sich im Prozess der Jahre durch diverse Einflüsse vertieft hat. Zunächst geplant als Evaluation zu Effektivität der deutschen Version eines niederländischen Projekts - einem 'Infokoffer' ${ }^{9}$, der anhand von wenigen sprachlichen Elementen die Möglichkeit bietet, die gegenwärtigen Versorgungsstrukturen 'ausländischen' Mitbürgern näher zu bringen - wurde durch die Beschäftigung mit den Themen 'Lernen', 'Stress', 'Belastung' und 'Trauma' eine neue Perspektive auf den Themenkomplex Migration eröffnet, zu der es bisher keine verknüpfenden Arbeiten gibt. Schließlich formte sich durch den Kontakt mit dem umfangreichen bestehenden GötTINGER KATALOG DiDAKTISCHER MODELLE (GKDM) die Idee, einen möglichen Beitrag zu eben jenem Katalog zu leisten und dabei auch auf Psychologie, Neurobiologie und unter anderem Soziologie zurück zu greifen, um einen didaktischen Vorschlag zu adaptieren und dessen Effektivität wissenschaftlich zu begründen. Das Fazit aus der ursprünglichen DissertationsKonzeption lautete: Bildungsveränderungen bei analphabetisierten Menschen sind schwer messbar, dennoch ist der Erfolg des Projektes unstrittig. Mit dieser Erkenntnis war die Basis für folgende Fragestellung geboren: Wie kann man (meist analphabetisierte) alte Menschen mit Zuwanderungsgeschichte dennoch unterstützen, zum einen zu lernen, zum anderen lernen zu Lernen und sie schließlich zudem besser mit Informationen zu versorgen?

\section{Erkenntnisinteresse, Zielsetzung und Vorgehen}

Zunächst wird knapp das Erkenntnisinteresse der vorliegenden Dissertation dargelegt, bevor im Anschluss die Strukturierung der Arbeit in aller Kürze erläutert und das Forschungsvorgehen, welches zu dieser Arbeit führte, reflektiert wird.

1. Erkenntnisinteresse und Zielsetzung: Durch Methodik kann in Deutschland gealterten Migranten zu einem „Wissens-“erwerb (ver-) helfen?

Wie einleitend bereits angeführt, stehen vier zentrale Begriffe im Fokus

9 Vgl.http://www.aelter-werden-

in.de//index.php?option=com_content\&task=view\&id=54\&ltemid=181, Zugriff am 17.06.2011 
dieser Arbeit, deren Verknüpfung und Betrachtung eines der Ziele ist: Migration, Alter, Lernen und Stress. Diese Begriffe sind für das Verständnis der vorliegenden Dissertation untrennbar miteinander verkettet, wie schematisch in folgendem Schaubild dargestellt ist.

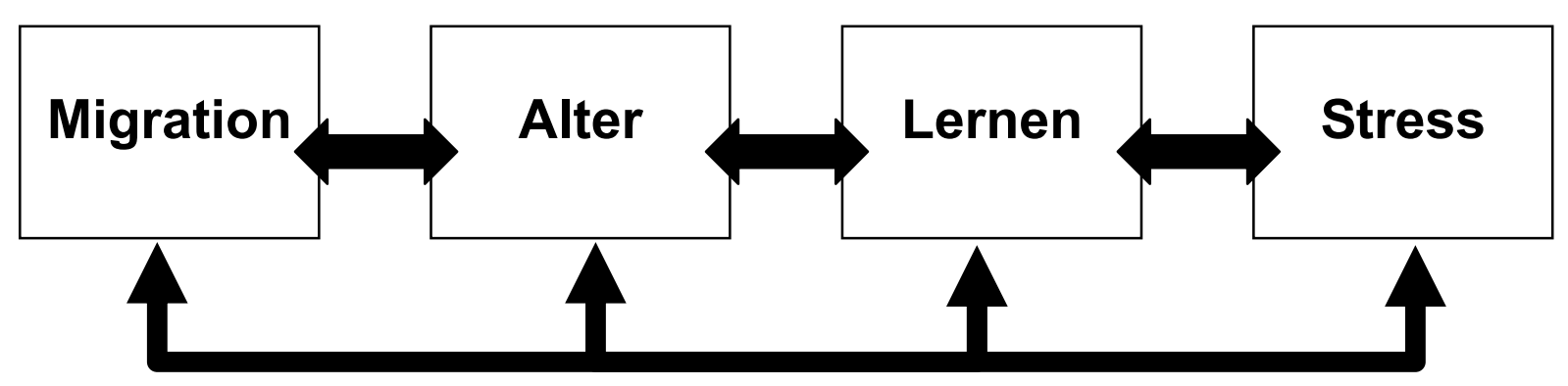

Diese Arbeit stellt erstmals eine Verknüpfung all dieser Aspekte dar, um sich der ganzheitlichen Betrachtung der Einzelthemen im Hinblick auf die anderen Einzelthemen nähern zu können. Gleichermaßen hat sie zum Ziel, im Fazit einen wissenschaftlichen Beitrag zum GötTinger KatAlog DidAKTISCHER MOdelLE zu leisten, um eine Lücke im Bereich der (gealterten) Erwachsenen(-bildung) zu schließen: den so genannten 'Infokoffer'. Dabei ist die Frage zentral: Wie kann man alten Migranten und Migrantinnen mit Sprachbarriere Wissen vermitteln und warum ist es wichtig, bei der Entwicklung eines solchen Konzeptes auf die Sprachbarriere, Lernkonzepte, das Alter oder den Migrationshintergrund und insbesondere Stressoren Rücksicht zu nehmen? Dabei werden primär Handlungsansätze für zukünftige Methoden und Forschungsbereiche hinsichtlich einer 'neu' erwachsenen Zielgruppe erschlossen.

\section{Vorgehen auf Basis der Grounded Theory}

Ausgehend von der Grounded Theory ${ }^{10}$ wird zunächst der aktuelle Forschungsstand angeführt. Die Grounded Theory ist eine inzwischen prominente, qualitative, hypothesengenerierende, sozialwissenschaftliche Forschungsmethode. Sie dient gleichermaßen als wissenschaftstheoretische

10 Vgl. insbesondere Jörg STRÜBING: Grounded Theory. Zur sozialtheoretischen und epistemologischen Dundierung des Verfahren der empirisch begründeten Theoriebilung. Wiesbaden 2008. 
Grundlage für die empirische Hypothesen- bzw. Konzeptentwicklung und ebenfalls auch als Fazit der Arbeit, da sich der Forschungsgegenstand mit zunehmendem Informationsgewinn in verschiedenen Gebieten (Neurobiologie, Kulturanthropologie etc.) stetig vertiefte und im Vergleich zum ursprünglichen Projekt prozessual gänzlich änderte. Da es bisher keine Arbeiten gibt, die sich thematisch mit allen Aspekten beschäftigen, die im Zentrum des Interesses dieser Arbeit stehen, wird zunächst der Forschungsstand aus den einzelnen Bereichen angeführt, insbesondere von Schnittstellenarbeiten, die sich wie folgt gliedern:

\begin{tabular}{|c|c|c|c|c|}
\hline & Migration & Alter & Lernen & Stress \\
\hline Migration & & 1 & 2 & 3 \\
\hline Alter & & & 4 & 5 \\
\hline Lernen & & & & 6 \\
\hline Stress & & & & \\
\hline
\end{tabular}

Nach dieser Literaturübersicht schließen sich in vier großen Kapiteln die Ausführungen zum jeweiligen Gegenstandsbereich an. Diese Ausführungen bauen jeweils auf dem vorherigen Kapitel auf und enden im Bereich der Neurobiologie. Dieser Zusammenhang wurde hinsichtlich Erklärungen von Migrationsstress und prozessen als Auswirkungen auf die Lernfähigkeit von Menschen mit Zuwanderungsgeschichte in diesem Sinne noch nicht veröffentlicht.

Am Anfang des Kernbereichs der Arbeit steht der Komplex 'Migration', welcher mit einer knappen Abhandlung über Kultur und die Auswirkungen auf kollektive und individuelle Identität eines Migranten eröffnet, bevor der Begriff Migration definiert wird und im Anschluss die Migrationsgeschichte sowie die Lebenssituation in Deutschland lebender Migranten dargestellt wird.

Da es sich bei dieser Arbeit hauptsächlich um in Deutschland gealterte Migranten handelt, schließt sich in logischer Konsequenz eine Definition des 'alten Menschen' an. Schließlich wird aus dem Bisherigen der Begriff des 'alten Migranten' entwickelt, der eine besondere Zielgruppe für das didaktische Modell des 'Infokoffers' darstellt.

Um pädagogische Mechanismen des Lernens verständlich zu machen, werden im nächsten großen Kapitel Lerntheorien erläutert, woraus sich dann später Besonderheiten für das Lernen im Alter unter Berücksichtigung aktueller Konzepte ableiten lassen werden. Um das Konzept des Arbeitens über Bilder und wenig sprachliche Mittel greifbar zu machen, erfolgt im Kapitel über das Lernen 
ein kleiner linguistischer Exkurs zum Thema 'Sprachliche Zeichen'. Im Anschluss wird die deutsche Adaption des niederländischen Infokoffer-Projekts erläutert und beschrieben, der als didaktisches Mittel für die erwähnte Zielgruppe im Fazit der Arbeit erneut Beachtung findet. Um diese Zusammenhänge schlüssig darzustellen, muss eine kurze generelle Abhandlung zur Didaktik folgen, um anschließend die Verbindung zum letzten Kapitel, der Neurobiologie und dem Themenkomplex 'Stress' herzustellen. Dieses letzte Kapitel stellt den Dreh- und Angelpunkt der vorliegenden Arbeit dar, da aus einer weiteren Disziplin heraus erklärt wird, wieso Menschen mit Zuwanderungsgeschichte in Deutschland Schwierigkeiten haben können sich zu integrieren und zu lernen.

Die Identitätsentwicklung der in Deutschland lebenden Migranten wird weiter unter dem Gesichtspunkt des 'Akkulturationsstresses' erörtert. Es werden hirnbiologische Zusammenhänge für die Lebenssituation der alten Migranten erarbeitet, um einerseits individuellen Stress dieser Menschen und andererseits Stress durch Migration als kollektive Erfahrung mit Folgen im 'Ankunftsland' in Einklang zu bringen.

Fallbeispiele aus der psychotherapeutischen Praxis als pathologisches Endstadium für die Unfähigkeit des 'Nicht-Lernen-könnens' werden als einer von zwei teilempirischen Aspekten folgen, um die vorangegangenen Erläuterungen exemplarisch zu belegen.

Mithilfe einer nicht-experimentellen bundesweiten Erhebung zur Lebenszufriedenheit (FLZ: Fragebogen zur Lebenszufriedenheit) bei ca. 60 Personen werden bisherige theoretische Überlegungen ebenfalls ergänzend belegt. Dazu werden statistische Daten über z.B. die Teilnahme an Deutsch- bzw. Integrationskursen und deren Abbruchraten bzw. erfolgreiche Abschlüsse herangezogen. Die Betrachtungen in den Kapiteln 'Migration', 'Alter', 'Lernen' und 'Neurobiologie'11 schließt im Kapitel 'Ergebnisse' mit den angeführten teilempirischen Erfahrungen und Erkenntnissen ab. Im Fazit werden die Zusammenhänge zwischen den Unterkapiteln nochmals erläutert und letztlich Elemente für zukünftige Entwicklungen von Methoden für die Arbeit mit in Deutschland lebenden älteren Migranten dargeboten. Als weitere Forschungsbereiche ergeben sich zusätzlich durch diese Arbeit Ansätze zur

11 Im Folgenden wird, wenn von Neurobiologie die Rede ist, gemeinhin Bezug genommen auf das Spezialthema Stress, da insbesondere die neuronalen Prozesse bei Stress für diese Arbeit von Relevanz sind. 
aktuell gesellschaftlich diskutierten Integration und dem Feld der biophysiologischen Untersuchungen an Migranten. Weiterhin können, auf Grundlage der in dieser Arbeit gewonnenen Erkenntnisse, mögliche politische Konsequenzen angedeutet werden. Im Ausblick werden weitere, sich aus der auf Basis der Grounded Theory erstellten Arbeit ergebende Überlegungen angeführt.

\section{B. Betrachtung der Zusammenhänge zwischen Migrationsstress, Alter und Lernen}

Im Folgenden werden auf Basis von Literatur aus den Bereichen Psychologie, Neurobiologie, Soziologie, Pädagogik, Kulturanthropologie und verwandten Wissenschaften Verknüpfungen zwischen den Themenbereichen Migration, Alter, Lernen und Stress aufgezeigt und schließlich auch neu geschaffen, um Hintergründe der eingangs dargestellten Problematik beleuchten und aus einem neuen Blickwinkel betrachten zu können.

\section{Bisheriger Forschungsstand aus den Bereichen Migration, Alter, Lernen und Stress}

Um den gegenwärtigen Forschungsstand als Basis für diese verknüpfende Arbeit nachvollziehen zu können, folgt nun eine Darstellung der (meist englischsprachigen) aktuellen Studien der jeweiligen thematischen Schnittstellen. Da es zum eigentlichen Thema der Dissertation noch keine ergiebige Fachliteratur gibt, folgt auf die knappe Vorstellung der aktuellen Literaturlage in den jeweiligen Kapiteln eine kurze Definition des Kapitelthemas, um diese Oberthemen im Fazit aufeinander beziehen zu können.

\section{Migration und Alter}

BRANDLEY/WILLIGEN (2010: 882-913) an, dass es zwar eine große Anzahl an Untersuchungen zur Migration älterer Erwachsener gibt, dass diese jedoch nur wenig Aufmerksamkeit auf die Auswirkungen der Migration auf die Migranten selbst richten. Ihre Untersuchung richtet ihr Augenmerk auf die Auswirkungen von Migration auf die depressive Symptomatologie bei älteren US-Amerikanern.

Sie kommen zu dem Ergebnis, dass die Auswirkungen von Migration von 
Person zu Person deutlich unterschiedlich verlaufen. Vor allem im Hinblick auf depressive Symptomatik legen die Ergebnisse der Untersuchung einen Zusammenhang nahe. Des Weiteren scheinen Wanderungsbewegungen im höheren Alter vor allem für Frauen und besonders mit zunehmendem Alter zu Stress zu führen.

ANGEL et al. (2010: 914-931) konnten in einer Untersuchung der Mortalität von mexikanisch-amerikanischen Immigranten im Alter von 65 Jahren und älter zeigen, dass diese in Zusammenhang mit dem Alter steht, in dem sich die Migration vollzogen hat. Dabei sind Migranten im reiferen Erwachsenenalter einem geringeren Sterblichkeitsrisiko ausgesetzt als solche, die in ihrer Kindheit oder im mittleren Lebensalter migrierten. Weder chronische Leiden, noch funktionelle Kapazitäten können diese Unterschiede erklären.

Etwas älter, aber nicht minder aktuell, ist SMITH CONWAYS und HOUTEVILLES Studie von 2003. Sie konnten in ihrer Untersuchung des Wanderungsverhaltens älterer Menschen aufzeigen, dass Unterschiede hinsichtlich der Entscheidungsmuster der 'Alten' und der 'jüngeren Alten' bestehen, was somit unterschiedliche Folgen für die Staaten hat, die sie bewohnen.

Dabei konnten SMith CONWAY und HoutENVILLE zeigen, dass alle älteren Migrantengruppen es vermeiden, in Staaten zu migrieren, welche hohe Grundbesitz-, Erbschafts- und Schenkungssteuern aufweisen, wobei dieser Effekt mit steigendem Alter abnimmt. Außerdem konnten sie darlegen, dass die jüngeren Alten eher Regionen aufsuchen, welche sich durch ein gemäßigtes Klima und eine vorteilhafte Politik in Hinblick auf Einkommenssteuer und soziale Fürsorge auszeichnet, wohingegen die älteren Alten eher durch hohe Lebenhaltungskosten und hohe Einkommens- und Grundstückssteuern aus ihren Herkunftsstaaten vertrieben werden.

Schließlich untersuchten WONG und GonZALEZ-GonZALEZ 2010 internationale Migration mit besonderem Augenmerk auf Behinderungen und Wohlstand aus der Perspektive des Herkunftslandes. Sie können dabei deutliche Unterschiede zwischen den Geschlechtern aufzeigen. So sind weibliche Rückkehrer nach Mexiko mit größerer Wahrscheinlichkeit behindert, wohingegen männliche Rückkehrer im Vergleich zu vergleichbaren älteren Erwachsenen vermögender sind. 


\section{Migration und Lernen}

Für einen analytisch-deduktiven Zugang ergibt sich aus der Matrix unter II. auch der Zusammenhang von Migration und Lernen. Trotz intensiver Recherche kann an dieser Stelle jedoch leider bisher keine Forschungsarbeit präsentiert werden. Die häufigsten Ergebnisse zum Thema Migration und Lernen belaufen sich lediglich auf die spezielle Thematik 'Lernen über Migration' oder auch Studien zur Migration von Software bzw. Computertechnologien. Von Interesse an dieser Stelle ist jedoch der Zusammenhang von Lernen und Migration, und somit sind gegenwärtig leider keine Arbeiten für das Erkenntnisinteresse dieser Arbeit nutzbar. Die vorliegende Dissertation kann künftig einen Baustein liefern, um diese Lücke zu schließen.

\section{Migration und Stress}

CHEKA et al. (2010: 109-116) konnten in ihrer empirischen Untersuchung von kurdischen Frauen mit (Zwangs-)Migrationserfahrung zeigen, dass posttraumatische Reaktionen in starkem Zusammenhang mit dem Zustand der mentalen Gesundheit stehen. Ebenso konnten sie einen signifikanten Zusammenhang zwischen dem Zustand der mentalen Gesundheit und der Erfahrung, vor Krieg und Unterdrückung geflohen zu sein, nachweisen, sowie den Zustand der inneren Vertriebenheit/Binnenvertriebenheit anführen. JÄKEL (2008: 15) untersuchte mittels des 'Everyday Stressors Index' nach Hall die unterschiedlichen Auswirkungen von täglichen Stressfaktoren auf die Lebenssituation von türkischstämmigen Müttern im Vergleich zu deutschen Müttern mit vergleichbarem Lebenshintergrund. Dabei zeigte sich, dass türkischstämmige Mütter mit einer 10-11-jährigen Schulbildung höhere Belastungswerte als deutsche Mütter mit ähnlichem Bildungsstand aufweisen. Ab einer Schulbildung von 12 und mehr Jahren waren diese Unterschiede nicht mehr zu finden. JÄKEL folgert daher, dass die Schulbildung als Indikator für den Zugang der türkischstämmigen Mütter zur Kultur und Sprache erkennt, eine dämpfende Wirkung auf die psychosoziale Belastung erwirkt. So steigt auch die Lebenszufriedenheit bei türkischstämmigen Müttern mit der Dauer des Schulbesuchs an.ebenso wirkt sich der Aufenthaltsstatus der türkischstämmigen Mütter positiv auf deren Lebenszufriedenheit aus. 


\section{Lernen und Alter}

An der Schnittstelle von Lernen und Alter finden sich gegenwärtig vier basale, für diese Arbeit relevante Studien. EPPINGER UND KRAY (2010: 41-52) untersuchten, ob ältere Erwachsene besser durch schlechte als durch gute Entscheidungen lernen, als dies bei jüngeren Erwachsenen der Fall ist und ob sich dies in der fehlerbezogenen Negativität (ERN = „Error-Related Negativity“) widerspiegelt. Die Auswertung des Verhaltens zeigte deutliche individuelle Unterschiede beim Lernen aus positiven und negativen Auswirkungen,welche durchweg stärker für ältere Erwachsene als für jüngere Erwachsene ausgeprägt sind. Ältere Negativ-Lerner zeigten eine stärkere Tendenz dahingehend, negative Folgen zu vermeiden, als jüngere Negativ-Lerner. Jedoch ist dieser Negativ-LernBias, im Gegensatz zum Fall von jüngeren Erwachsenen, nicht in Zusammenhang mit einer erhöhten fehlerbezogenen Negativität zu setzen. Nach EPPINGER und KRAY legt dies nahe, dass das Vermeidungslernen bei älteren Negativ-Lernern möglicherweise von der Fehlerverarbeitung entkoppelt ist. Des Weiteren zeigen ältere Erwachsene im Vergleich zu jüngeren Erwachsenen Lernschwächen. Die ERP Analyse deutet darauf hin, dass diese Beeinträchtigungen Defizite in der Fähigkeit zum Aufbau relationaler Darstellungen von mehrdeutigen Ergebnissen widerspiegeln. Im gleichen Jahr veröffentlichten HÄMMERER et al. (2010: 579-592) ihre Studie. Sie haben die Auswirkungen von ErgebnisüberwachungsMechanismen auf altersbezogene Unterschiede hinsichtlich probabilistischem Verstärkungslernen untersucht, indem sie die Feedback-bezogene Negativität (FRN= Feedback-Related Negativity) als Reaktion auf Gewinne und Verluste aufgezeichnet haben. Im Detail haben HÄMMERER et al. die Unterschiede der Beobachtungsreaktionen auf Gewinn und Verlust festgestellt, um die Beobachtung von Ergebnissen (monitoring of outcomes) gemäß aufgabenspezifischer Ziele über die Lebenszeit hinweg zu untersuchen. Die Amplitude der FRN nach Gewinnen und Verlusten nimmt von der Kindheit ins hohe Alter hinweg monoton ab. Des weiteren wiesen Kinder und Alte im Vergleich zu Adoleszenten und jüngeren Erwachsenen geringere Unterschiede zwischen der FRN nach Gewinnen und der FRN nach Verlusten auf, was HÄMMERER et al. als eine weniger differenzierte Ergebnisklassifikation auf Basis von aufgabenspezifischen Zielen interpretieren. Ebenso bedurften Kinder und Alte mehr Versuche, um aus den Ergebnissen von Entscheidungen zu lernen, insbesondere wenn die 
Belohnungswahrscheinlichkeit verschiedener Wahlmöglichkeiten nur gering voneinander abwich. Außerdem zeigte sich, dass Kinder und Alte durch Belohnungen weniger lernten als durch Verluste.

SCHULZ Und ROBNAGEL (2010: 383-399) untersuchten, ob Lernkompetenzvariablen den Erfolg von informellem Lernen vorhersagen können. Dabei stellte sich heraus, dass Lernkompetenz den Erfolg von informellem Lernen unabhängig vom Arbeiteralter vorhersagen kann, wobei eine teilweise mediierende Wirkung von Gedächtnis und Selbstwirksamkeit zum Tragen kommt, welche möglicherweise zweitrangige Alterseffekte unter bestimmten ungünstigen T\&D Bedingungen induzieren kann.

SKOLIMOWSKA et al. (2010: 87-93) haben altersbezogene Unterschiede hinsichtlich zweierlei Arten von räumlichem Erinnerungsvermögen anhand des PAL (paarweises verbundenes Lernen) Testverfahrens aus dem CANTABand und über den RIM-Test unter Anwendung von lebensechten Settings untersucht.

Ältere Testsubjekte zeigten schlechtere Leistungen als ihre jüngeren Pendants beim PAL-Test, was für alle getesteten Ergebnis-Maße nachgewiesen wurde. Im Gegensatz dazu wurde im RIM-Test kein klarer Alterseffekt beobachtet. Ein Einfluss auf das Ergebnis durch das Geschlecht konnte in keinem der Tests gezeigt werden. Eine Disassoziation der altersbezogenen Veränderungen bei diesen beiden Tests deutet darauf hin, dass das visuell-räumliche assoziative Lernen und die ideothetische Navigation möglicherweise unterschiedliche kognitive Kontrollen aufweisen, welche vermutlich in einem Zusammenspiel unterschiedlicher Gehirnstrukturen begründet liegen.

\section{Alter und Stress}

DIEHL und HAY (2010) untersuchten in ihrer Studie folgende drei Hypothesen:

- die Unabhängigkeit der Reaktivität auf täglichen Stress vom Alter,

- eine mögliche moderierende Wirkung des Alters auf den Zusammenhang zwischen Selbstbild- Inkohärenzen und der Reaktivität auf täglichen Stress sowie

- einen dämpfenden Effekt der wahrgenommenen Selbstkontrolle auf die Reaktivität auf täglichen Stress, welcher für junge Erwachsene deutlich 
stärker sein sollte als für Erwachsene mittleren und höheren Alters.

Als Ergebnis konnten die beiden Forscher aufzeigen, dass das tägliche Wohlbefinden durch verschiedene Risiko- und Spannungsfaktoren auf komplexe Weise beeinflusst wird. Dies gilt sowohl für das Wohlbefinden im Allgemeinen, als auch für das Wohlbefinden hinsichtlich der Stressreaktivität. Die Untersuchungsergebnisse von DIEHL und HAY legen nahe, dass jüngere Erwachsene und solche mit größeren Inkohärenzen im Selbstbild stärkere emotionale Instabilität während der Untersuchung erlebten und auch insgesamt einen größeren Durchschnitt hinsichtlich des negativen Affekts aufweisen.

Obwohl erwachsene Probanden einen größeren negativen Affekt an Tagenmit erhöhtem Stress vermerkten, wirkten sich Alter und SelbstbildInkohärenzen nicht auf die Stressreaktivität aus. Vielmehr wurde diese durch die wahrgenommene Selbstkontrolle abgeschwächt, vor allem bei jüngeren Erwachsenen.

Zusammenfassend legen DIEHLS und HAYS Studie nahe, dass persönlichkeitsbezogene Risiko- und Spannungsfaktoren das tägliche Wohlbefinden, die emotionale Stabilität von Tag zu Tag und die Stressreaktivität in unterschiedlichem Maße über das Erwachsenenalter hinweg beeinflussen.

NEUPERT et al. (2007) stellen die Hypothesen auf, dass zunehmendes Alter in Zusammenhang mit einem höheren Niveau täglichen emotionalen Wohlbefindens und einer reduzierten Reaktivität auf tägliche Stressoren steht. Des Weiteren postulieren sie, dass sich höhere Stufen der Persönlichkeitsentwicklung (genauer: der persönlichen Meisterschaft) und weniger Zwänge positiv auf das physische Wohlbefinden auswirken und die Reaktivität auf tägliche Stressoren senken. Dies gilt über alle Altersgruppen hinweg.

NEUPERT et al. legten außerdem die Hypothese nahe, dass sich die Ausprägung von Kontrollüberzeugungen bezüglich der Reaktivität auf Stressoren sich mit dem Alter unterscheidet. Diese Unterschiede stellen sie derart dar, dass Kontrollüberzeugungen vor allem auf die Altersgruppen für welche die Dominanz der Stressoren besonders salient ist große Auswirkungen haben,.

In Bereichen, in denen die Salienz nicht systematisch mit dem Alter in Zusammenhang steht, also beispielsweise im Bereich von Heim und Netzwerk, haben NEUPERT et al. Unterschiede bei Kontrollüberzeugungen bezüglich der 
Reaktivität untersucht.

Bei der Untersuchung dieser Hypothesen haben NEUPERT et al. entdeckt, dass Erwachsene mittleren und höheren Alters körperlich und emotional auf interpersonale Stressoren weniger stark reagieren. Auch zeigen Erwachsene mittleren Alters insgesamt weniger körperliche Reaktionen auf Arbeitsstressoren. Ebenfalls dokumentieren NEUPERT et al. den heilsamen Effekt von wenig wahrgenommenen Zwängen und einer großen persönlichen Meisterschaft ${ }^{12}$, durch die Abschwächung emotionaler und körperlicher Auswirkungen von täglichen, interpersonalen, netzwerkbezogenen und arbeitsbezogenen Stressoren.

Des Weiteren haben NEUPERT et al. belegen können, dass in dem Sinne die Auswirkungen von geringer wahrgenommener Kontrolle über das Alter hinweg in unterschiedlichem Ausmaß wirksam sind, sodass diese sich für jüngere Erwachsene weniger abträglich im Kontext zu interpersonalen Stressoren als sie es für Erwachsene mittleren und höheren Alters gestalten.

Bezüglich der Auswirkung von Arbeitsstressoren konnte gezeigt werden, dass geringe Meisterschaft über alle Altersgruppen hinweg abträglich ist, wobei für Erwachsene mittleren Alters auch gilt, dass sie trotz großer Meisterschaft körperliche Reaktionen auf Arbeitsstressoren zeigen. Zwänge können vor allem in Zusammenhang mit verstärkter körperlicher Reaktivität auf netzwerkbezogene Stressoren gebracht werden, wobei besonders die Reaktivität von jungen und alten Erwachsenen verschärft wird. Des Weiteren zeigen junge und ältere Erwachsene mit geringer Meisterschaft gesteigerte emotionale Reaktivität auf Netzwerkstressoren. NEUPERT et al. interpretieren diese Ergebnisse als Auswirkungen von Lebensumständen und Stressdominanz, welche sich im Laufe des Alterns verändern.

TROUILLET et al. (2009: 357-366) machten es sich zur Aufgabe, die Widersprüche bezüglich der Veränderung von Coping mit dem Alter zu überprüfen. Sie postulieren, dass die Veränderung von Coping mit dem Älterwerden über Coping-Ressourcen, wie Selbstwirksamkeit, wahrgenommenen Stress und die Zufriedenheit mit sozialer Unterstützung, mediiert wird.

Als Ergebnis ihrer Studie konnten sie ein modifiziertes Modell zu den Auswirkungen von Alter auf Copingstrategien bestätigen, in dessen Kern sich das

\footnotetext{
${ }^{12}$ Im englischen Originaltext als Fachbegriff der Autoren eingeführt und mit ,mastership' bezeichnet.
} 
Alter direkt auf Prozesse des Copings auswirkt. Das Alter selbst konnte weder ein problemzentriertes, noch ein emotionszentriertes Coping vorhersagen. Stattdessen stellten sich Selbstwirksamkeit und die Zufriedenheit mit sozialer Unterstützung als Prädiktor für ein problemzentriertes Coping und die Zufriedenheit mit sozialer Unterstützung, sowie wahrgenommener Stress als Prädiktor für emotionszentriertes Coping heraus.

\section{Stress und Lernen}

CALANDREAU et al. (2011: 104-110) untersuchten jüngst die Auswirkungen von kurzen Stressphasen auf das Lernverhalten von japanischen Wachteln und konnten dabei zeigen, dass kurze Phasen während denen die Wachteln unvorhersehbar und wiederholt über einen Zeitraum von 8 Tagen mit aversiven Ereignissen konfrontiert wurden, deren räumliches Lernverhalten gegenüber der Kontrollgruppe nicht verbessern_konnte. Dass diese Erfahrung von den Wachteln als Stress wahrgenommen wurde, schließen die Untersuchenden aus Gewichtsunterschieden zwischen den behandelten Wachteln und einer Kontrollgruppe, auch wenn sie keine Unterschiede hinsichtlich der Korticosteronwerte zwischen den Gruppen ausmachen konnten. Damit schließen sie auf einen Zusammenhang zwischen der Dauer von Stressbelastung und deren negativer Konsequenzen auf das Lernverhalten von Wachteln, wie es in einer vorangegangen Studie belegt wurde.

SHORES (2006: 55-85) konnte zeigen, dass Stresserfahrungen vor allem solches Lernen verbessern können, welches in den Bereich von Angst und Lidschlag- Konditionierung fällt, wie auch das Lernen über bedrohliche Stimuli. Stresserfahrungen im Lebensverlauf wirken sich auf die Gedächtnisleistung der Betroffenen aus. SHORES merkt an, dass bislang nur wenig Aussagen zu einem Trendverlauf zwischen Stress und Lernverhalten getroffen werden können, da vor allem erwachsene Männer untersucht wurden, obgleich die Auswirkungen von Stress auf das Lernverhalten durch Alter und Geschlecht beeinflusst werden. Bezüglich der Mechanismen und des anatomischen Substrats beschreibt SHORES die Auswirkungen von Stress auf das Lernen als abhängig von Stresshormonen, in Zusammenhang mit neuronaler Aktivität im Hippocampus, der Amygdala, des bed nucleus, der stria terminalis und des präfrontalen Cortex. 
Abschließend ist somit festzuhalten, dass die Studienlage der thematischen Schnittstellen hilfreich ist und somit als fundierte Basis für die vorliegende Arbeit nutzbar gemacht werden kann. Sie weist jedoch noch die Lücke der Verknüpfung der jeweiligen Aspekte auf, die diese Arbeit in Ansätzen zu schließen sucht. Im folgenden Kapitel werden auf dieser Basis die Themen Migration, Alter, Lernen und Stress vertieft behandelt.

\section{Betrachtung der Elemente: Migration - Alter - Lernen und Stress}

Die gesammelten Erkenntnisse des gegenwärtigen Forschungsstandes dienen im Folgenden als Fundament. Diese Grundlage wird durch vertiefende Aspekte ergänzt, die final zu einem Modellentwurf für einen didaktischen Ansatz führen können, der es (alten) Migranten möglich macht (wieder) zu lernen. Um die Thematik und auch die mit selbiger verbundene Problematik des gesamten Komplexes 'Migration' hinreichend nachvollziehen und verstehen zu können, ist es nötig sich zunächst vor Augen zu führen, was Migration meint.

\section{Migration}

Ein wichtiges Element, dass das Heimatland vom so genannten Ankunftsland unterscheidet, ist die Kultur aus der die Migranten kommen, und in welche sie hinein wandern. Um zu verstehen, wie es zu kulturellen Differenzen kommen kann und was „kulturangepasste Methoden“ sein können, um den Migranten das Lernen zu erleichtern, ist es zwingend nötig sich eingangs zu fragen: Kultur - was ist das?

\subsection{Am Anfang steht die Kultur - was ist das?}

Die Frage nach dem, was Kultur ist, sein kann oder sein soll stellen sich Kulturanthropologen regelmäßig und es fällt ad hoc nicht leicht, diese Frage zu beantworten. Dass Kultur jedoch elementar ist, scheint unstrittig: 
„Menschen nur auf ihre Kultur zu reduzieren, als auch, sie ohne ihre kulturelle Herkunft zu betrachten, würde bedeuten, einen Irrweg einzuschlagen“ (ATABAY 2010: 129).

Weit komplizierter ist es Kultur beispielsweise etymologisch zu erklären. Der deutsche Begriff ist dem Lateinischen 'cultura' entlehnt. Dieses Nomen geht auf das Verb 'colere' zurück, das zwei Bedeutungen inne hat: drehen, wenden, bebauen (des Ackers) - es bedeutet jedoch zudem auch anbeten. Parallel zur Pflege des Ackers ist dem lateinischen Dichterfürsten Cicero der Begriff der Seelenpflege, der „Cultura animi' zu verdanken. ${ }^{13 .}$ Seit der UNESCOKulturkonferenz in Mexiko 1982, wird international eine an anthropologischen und ethnologischen Begrifflichkeiten angelehnte Definition von Kultur benutzt, die dem Grundverständnis der vorliegenden Arbeit entspricht und daher fortlaufend als Kulturbegriff genutzt wird:

Die Kultur kann in ihrem weitesten Sinne als die Gesamtheit der einzigartigen geistigen, materiellen, intellektuellen und emotionalen Aspekte angesehen werden, die eine Gesellschaft oder eine soziale Gruppe kennzeichnen. Dies schließt nicht nur Kunst und Literatur ein, sondern auch Lebensformen, die Grundrechte des Menschen, Wertesysteme, Traditionen und Glaubensrichtungen. UNESCO 1982

Weit umfassender und dennoch dem obigen Inhalt weitestgehend entsprechend findet sich eine Definition von Kultur auch in folgendem längerem Glossar, das beispielsweise auch explizit auf den Islam hinweist, der als primär praktizierte Religion für türkischstämmige Migranten eine Rolle spielt:

'identity only becomes an issue when it is in crisis, when something assumed to be fixed, coherent and stable is displaced by the experience of doubt and uncertainty' (Mercer 1994: 259).

In broad terms, this uncertainty can be explained by two keys: major social and economic developments. First, the events in Europe of 1989 undermined received identities just as they undermined geographical and political borders within European states and between former communist and western social democratic regimes. Second, GLOBALIZATION has increased the experience of migrancy, altering relations between western and other cultures, and the sense of identity of individuals whose lives have taken them across the borders between First, Second, and Third Worlds, or across, in effect, pre-modern and postmodern societies.

BROOKER (2003) erläutert, dass der Zusammenbruch des ehemaligen kommunistischen Regimes und die Globalisierung zu Erlebnissen von Unsicherheit und Zweifeln führen. Beide Entwicklungen haben die Identitäten der Betroffenen herausgefordert oder verändert. Er betont jedoch auch, dass die Folgen für die Identitäten mehrschichtig und teilweise gegenläufig sind, insofern als sie die alten, etablierten Identitäten als Kommunisten oder Demokraten

13 Tusculanae disputationes Buch 2, 5, 13 
aufgeweicht haben, zugleich aber auch zu einem Wiederaufleben von traditionellen ethnischen Identitäten geführt haben (als Beispiel nennt er hier etwa die Entwicklungen im ehemaligen Jugoslawien):

At the same time these developments are themselves ambiguous and incomplete.

For while they have promoted a relaxation of fixed and coherent identities, this has been accompanied by contrary tendencies: the revival in eastern Europe of traditional ethnic identities (of Bosnian Serb and Croat in the former Yugoslavia, for example) and a more emphatic construction of individuals as consumers for the ever-expanding markets that characterize globalization. BROOKER (2003: 130-132)

Für die aktuelle Situation postuliert er entsprechend, dass zeitgenössische Identitäten wandelbar und bewusst begrenzt sein können, da die konstituierenden Faktoren, seien diese nun Religion, Nation, Sprache, politische Ideologie oder kulturelle Ausdrucksweise, allesamt verhandelbar sind:

Contemporary identities can therefore be fluid or consciously delimited. Any number of factors are likely to be under NEGOTIATION in either case; whether of religion, nation, language, political IDEOLOGY or cultural expression. Islam, for example, is a religious faith that shapes the social, economic and political character of entire regimes and can reach into the detailed social and sexual lives of its adherents. In Northern Ireland, religious faith combines with political belief in the continued enmity between Catholic and Protestant communities.

Often, too, such identities are reinforced in further relations beyond their immediate definition, as in the divided allegiances of Northern Ireland communities to the implications of Britishness - itself a source of ambivalent identity and a topic of dispute on the mainland (see Storry and Childs 1997). Identities of these and other kinds - of generation and sexual orientation, for example - are also expressed in symbolic cultural forms: in the adoption of national dress or musical styles (from rap to the marching bands of the Irish Apprentice Boys); in a certain diet (kosher, vegan, junk food); in the symbolic coding of hairstyles or footwear; in the exposure or concealment of body parts, and so on. BROOKER (2003: 130132)

BROOKER (2003) spricht hier auch die formende Kraft von Glaubensrichtungen, explizit die des Islams an: er weist darauf hin, dass der soziale, ökonomische und politische Charakter eines Regimes durch diese Glaubensrichtung geformt werden kann und weiter, dass sich ihr Einfluss bis auf die Ebene der individuellen Anhänger hinsichtlich ihres sozialen und sexuellen Lebens vollzieht.

Er unterstreicht, dass Identitäten, die von Religion, Generation oder sexueller Orientierung o.a. geprägt sind, auch Ausdruck in verschiedenen symbolischen Kulturformen, wie etwa einer bestimmten Kleidung oder Musik, einer besonderen Ernährungsweise usw. finden.

Developments in theory have accompanied the general social processes indicated above and have played their part in underlining, and providing a vocabulary for, a changing 
awareness of the many subtleties of identity. An early influential development within poststructuralist FEMINISM and latterly the study of RACE was the CRITIQUE of essentialist notions of identity (of being a 'woman' or a 'black man') and of the allied affirmation of a given identity in relation to its supposed binary opposite. As Henrietta Moore remarks, 'the assertion of the non-universal status of the category "woman" is by now almost a commonplace' (in Woodward [ed.] 1997: 60). This critique has been consolidated in a recognition of the overlapping and inconsistent alignment of additional factors of class, race, ethnicity, sexuality, age and so on. Being a black, lesbian, mother born in New York and living in Paris, for example, suggests a fluid personal, ethnic, linguistic and cultural identity, where no one factor predominates. Where one factor does predominate or is chosen as a description governing an individual or group's actions (i.e. being a woman, being Serbian, Palestinian, black or gay) this is termed 'identity polities' (see Hobsbawm 1996). There can be little doubt that contemporary cultural theory has sought to challenge or deconstruct essentialist, universalizing or fundamentalist identities.

This has given currency to concepts of DIFFERENCE, HYBRIDITY, migrancy and the DIASPORA (Gilroy 1993; Bhabha 1994; Morley and Chen [eds] 1996) and a preference for these terms over conceptions of settled ethnic, national or geographical identities. Obviously this is not simply a theoretical preference but an aspect of contemporary CULTURAL POLITICS, presented in the name of a better, because more open, pluralist and tolerant, world. BROOKER (2003: 130-132)

In seinen weiteren Ausführungen stellt BROOKER die Entwicklungen der wissenschafltichen Theorien unter Hervorhebung des Einflusses des poststrukturalistischen Feminismus und der in diesem enthaltenen Kritik am essentialistischen Identitätsbegriff, dar. Diese Theorien erachtet er dafür entscheidend, dass sich die zeitgenössische Kulturtheorie um die Dekonstruierung von essentialistischen, universalisierenden oder auch fundamentalistischen Identitäten bemüht.

Und weiter hat eben diese Entwicklung der wissenschaftlichen Theorien es erst ermöglicht, die Konzepte von Differenzen, Hybridität, Migration und Diaspora zu fassen und diese darüber hinaus gegenüber den Vorstellungen sesshafter ethnischer, nationaler oder geographischer Identitäten zu bevorzugen.

Diese Präferenz ist jedoch nicht nur in der wissenschafltichen Theorie, sondern auch in der zeitgenössischen Kulturpolitik zu finden.

A politics of this kind should be distinguished from the sectional exclusivity of 'identity polities' of a group or nation and from a traditional Left politics based on a universalizing, class-based identity. It must be said, all the same, that traditional or essentialist identities cannot be wished or written away. They similarly answer to real needs and often to fears of subjection and the loss of identity:

precisely, in their own terms, to the 'experience of doubt and uncertainty' Kobena Mercer describes above. It is this complexity of response that makes identity a topical and important issue. (Brooker 2003: 130-132)

BROOKERS Zitat schließt mit dem Aufruf, nicht dem Irrglauben zu verfallen, dass das Auftauchen dieser neuen Arten von Identität die traditionellen bzw. 
essentialistischen Identitäten hätte verschwinden lassen. Schließlich können sie nach wie vor realen Bedürfnissen und Ängsten vor Abhängigkeit oder Identitätsverlust begegnen, also eben den eingangs des Zitats beschriebenen Zweifels- und Unsicherheitserlebnissen.

Zusammenfassend wird aus dem oben genannten Zitat von BROOKER ersichtlich, dass die Identität in neueren theoretischen Ansätzen durch eine Vielzahl verschiedener Faktoren beeinflusst wird bzw. beeinflusst werden kann.

Daraus ergeben sich für die Betrachtung von Kultur einige Konsequenzen, unter anderem dergestalt, dass durch die Globalisierung bzw. durch den Aspekt der globalen Bewegungsflexibilität, der diese ja charakterisiert, 'klassische' ethnische, nationale oder auch geographische Identitäten nicht mehr im Zentrum der Kulturbetrachtung stehen. Vielmehr wird nun die Bedeutsamkeit der persönlichen Biographie- und Migrationsgeschichte, die sich auf die politische Haltung, die Kultur, die Herausbildung von Vorurteilen, das Verständnis und die individuelle Bedeutung von Religion, Ethnizität, Unterschiedlichkeit und Hybridität, somit die sich aus der Lebensgeschichte ergebende ambivalente Identität der Migranten deutlich. Diese ambivalente Identität dient letztlich als symbolhafte kulturelle Darstellung.

Näher eingehend auf den Migrations- und Biografieaspekt in Hinsicht auf Kultur, ist Kultur nach FRÖLICH (2003) als „bereits in ihrem Ursprung ein Ort des unentwegten Widerstreits zwischen Repräsentation von Welt, Subjekt und Geschichte“. (2003: 45)

Hier werden also Zusammenhäge von Bewusstheitsprozessen, Wahrnehmung, Subjektivität und der Prägung von Identität angedeutet. Kulturbetrachtungen im vergleichenenden Kontext weisen auch immer Schnittstellen mit globaler Bewegungsflexibilität auf. Gleichzeitig entsteht Vorurteilsbildung (aus welcher sich eine politische Haltung entwickeln kann gegenüber den vermeintlich offenkundigen kulturellen Unterschieden wie Religion, Ehtnizität, Unterscheidlichkeit, Hybridität, ambivalente Identität etc.), die 'Kultursymbole' entwickeln. Zum Beispiel wird dann, um sich seiner Kulturzugehörigkeit versichert zu sein, der kulturell dominanten Gesellschaft die religiöse Andersartigkeit deutlicher vor Augen geführt. 
Auch das folgende Zitat, das sich auf die Erzählung "Mutterzunge“ von ÖZDAMAR (1991) bezieht, zeigt, wie stark sich einzelne Aspekte der Identität (in diesem Fall die Muttersprache) auf die Kulturzugehörigkeit auswirken.

In dem unten geschilderten Fall, wird deutlich, dass für die Protagonistin der Verlust der früheren Identität bzw. die Wirren um die eigene Identität nach der Migration, durch den Verlust der Muttersprache, das Verlernen dieser, wahrnehmbar wird:

\begin{abstract}
Fern von Istanbul wird die in Berlin lebende und zwischen westlichem und östlichen Teil der deutschen Metropole hin und her pendelnde Ich - Erzählerin von der Frage umher getrieben, wann sie wohl ihre "Mutterzunge“ verloren haben mag. In ihrer Erinnerung klingen die Sätze der Mutter „wie eine (...) Gut gelernte Fremdsprachen“ (Mutterzunge, S.7). Zwar beinhaltet die Migration und das Leben in der Fremdsprache die Entfremdung von Herkunftsort und Muttersprache und stellt so für die Erzählerin den Auslöser dar, über Sprache, Identität und den Verlust der Muttersprache nachzudenken. Doch löst Özdamer Letzteren aus einem unmittelbaren kausalen Zusammenhang mit der Migration und führt inn auf ein ganz anderes, die zwar weniger in geographischer Hinsicht bedeutsames, der vernichtende einschneidendes kulturpolitisches Ereignis in der Geschichte der Türkei zurück. [...] Danach habe die Abschaffung der arabischen Schrift das kollektive vergessen der kulturellen Vergangenheit der Türkei eingeleitet, indem sie die Wurzeln des islamisch ottomanischen Erbes gekappt und dieses nachfolgenden Generation unzugänglich gemacht habe: „dieses Verbot ist so, wie wenn die Hälfte Von meinem Kopf abgeschnitten ist“, lässt ÖZDAMAR ihre Erzählerin sagen." (50-51)
\end{abstract}

Demnach entsteht Kultur auch aus der Muttersprache., Diese ist elementarer Bestandteil des Enkulturationsprozesses - also des Hineinwachsens und Eingeführtwerdens in eine Kultur.

Im Türkischen ist eines der primären Artikulations'organe', die für die Sprachübermittlung zuständig sind - also die Zunge (lat. lingua; auch lat. für Sprache) - Teil des Wortes, das im Deutschen mit Muttersprache übersetzt wird: 'Die Mutterzunge'. Die Identifikation, wie die der Protagonistin obigen Werks, mit der Muttersprache trägt also stark zur Identitätsbildung bei. Ein Verlust dieser 'Mutterzunge' jedoch führt zu Identitätsverwirrung, da sich die Kulturzugehörigkeit unter anderem durch die Sprache 'empfinden' lassen kann. Zusammenfassend kann man also sagen: Die Kultur entsteht hier aus der Muttersprache und wird ein aus der subjektiven Identität geschaffenes Objekt, hier genauer aus der nicht greifbaren Sprache und der Lingua, die gemeinsam zum Objekt „Mutterzunge“ werden, mit deren Verlust auch die Identität unsicher wird. Bedeutsam ist an diesem Zitat auch der Verweis auf das kollektive Vergessen der kulturellen Vergangenheit. Auf die besondere Bedeutung des kollektiven und des kulturellen Gedächtnisses wird im Folgenden noch eingegangen werden. 


\section{2 (Kulturelle) Identität}

In der populären Parodie 'Das Leben des Brian' ${ }^{14}$ der Comedy-Formation 'Monty Python' wird auf satirische Weise das Thema der Identität von Individuen dargestellt. Auf die Aussage des Protagonisten Brian 'Ihr seid doch alle Individuuen' und 'Und ihr seid alle völlig verschieden' antwortet das versammelte Volk chorisch 'Ja, wir sind doch alle Individuuen' und 'Ja, wir sind alle völlig verschieden' und parodieren somit die Individualitätsbehauptung. Bis ein Individuum der versammelten Anhängerschaft(-sherde) konstatiert: 'Ich nicht', woraufhin inn das Volk zum Schweigen verpflichtet. Die Parodie gipfelt an dieser Stelle. Hierbei handelt es sich um eine von beispielsweise drei Identitäsformen, die exemplarisch einleitend genannt werden sollen: Soziale Identität, Nationale Identität und Duale Identiät.

Unter 'Sozialer Identität' versteht man gemeinhin die Definition eines Individuums über sein soziales Umfeld. Dabei können Mitgliedschaften im Sportverein, Kohortenzugehörigkeit, subkulturelle Zugehörigkeitsgefühle wie z.B. zu bestimmten Musikszenen wie Punk-Rock gemeint sein, aber auch religiöse und spirituelle oder Interessens-Gemeinschaften. Die Theorie der 'Sozialen Identität' (TAJFEL / TURNER 1979, TAJfEL 1982) sowie die Theorie der Selbstkategorisierung (TURNER et al. 1982) bietet hierfür einen bekannten, belegten Hintergrund. Nach TURNER kann Soziale Identität somit als Gefühl der Zugehörigkeit eines Individuums zu einer sozialen Gruppe oder Kategorie jedweder Coleur gelten, sofern diese als bedeutsam von dem Individuum wahrgenommen (und auch empfunden) wird (vgl. TURNER 1982). Nach HOGg et. al (HOGg UND ABRAMS, 1988, zit. n. Hogg Und VAUGHAN, 2008; TAJFEL, 1981a; TAJfEL, 1982; TAJFEL UND TURNER, 1979; TURNER et al., 1987) tendieren Individuuen dazu, ihre Identität zum Teil aus dem Gruppenzugehörigkeitsgefühl abzuleiten, ebenso wie sich die Identifikation und Identiät von Gruppen entsprechend intragrupal vollzieht. Nach HogG et al. (vgl. Hogg / ABRAMS 1988; TAFEL / TURNER 1979) ist weiterhin zu beobachten, dass die Gruppenmitglieder im intergrupalen Identifikationskontext eine Tendenz zeigen, die eigene Gruppe als vorrangig positiv und distinkt wahrzunehmen, vor allem in Abgrenzung zu anderen Gruppen und damit das Identitätsgefühl potenzieren.

14 Monty Python: Das Leben des Brian. Orginaltitel 'Monty Python's Life of Brian'. Regie: Terry Jones. Großbritannien 1979. 
Final kann dieses Verhalten zu Überidentifikation führen, oder auch zu parteiischen Verhaltensweisen und Haltungen.

Bezieht sich dieses parteiische Verhalten nun beispielsweise auf eine Gruppe, deren verbindendens Element primär die Nationalität ist, nähert man sich dem Begriff der Nationalen Identität an. Die Selbstkategorisierungstheorie (TURNER, 1982, 1985, zit. n. HOgG UND VAUGHAN, 2008; TURNER et al., 1987; TURNER, OAKES, HASLAM UND MCGARTY, 1994) zeigt auf, dass sich Mitglieder einer Gruppe bei der nationalen Identiät - also die Identifikation mit einer Nation (eine Form der sozialen Identiät) - tendenziell selbst stereotypisieren und sich per Eigenschaften beschreiben, die typisch für die Identifikationsgruppe sind. Im Fokus steht für die Gruppenmitglieder hier auch, die eigene Nation als möglichst positiv von anderen Nationen abzugrenzen. Dies kann bei Überidentifikation zu Nationalismus führen. So wie sich bei der sozialen Identität Sportvereine als Beispiel anbieten würden, bietet sich für das Phänomen der Nationalen Identität beispielsweise Fußball als Sportart an, bei der nationale Identifikation stark beobachtet werden kann: 'Wir Bayern-München-Fans sind...Ihr HSV-Fans dagegen seid...'. Dass aus dieser inneren Haltung eine Veränderung der äußeren Verhaltensweisen im Sinne der Gruppenkultur und deren Werte und Normen erwächst, scheint logisch. Für die vorliegende Arbeit ist nun jedoch insbesondere die Frage interessant, was passiert bei Menschen mit mehr als einer oder dem Gefühl von keiner nationalen Zugehörigkeit: Wie verhält es sich mit der nationalen und sozialen Identiät bei Migranten?

Da Individuen generell über multiple Identitäten verfügen (SMITH 2011; VERKUYTEN 2005), weil sie sich grundsätzlich mit mehreren sozialen Gruppen identifizieren können, die aus den verschiedensten lebensweltlichen Bereichen stammen können, liegt die Frage nahe, ob eine Identifikation mit mehreren Nationen ebenfalls möglich ist. Selbst Menschen ohne Migrationshintergrund, die regelmäßig beispielsweise in ein spezifisches Urlaubsland fahren, können eine Identifikation mit der dortigen Nation haben. Dabei befinden sie sich nicht gemeinhin unter Stress, anders verhält es sich jedoch bei Menschen mit Zuwanderungsgeschichte. Hier spricht man, wenn eine Identifikation mit der Ursprungs- und Zielnation erfolgt, von Dualen Identitäten. Dabei handelt es sich 
jedoch nicht um ein gelegentliches und seltenes Phänomen, sondern um den lebensweltlichen Altag. Nach RUHS (2008) identifiziert sich die Zielgruppe dieser Arbeit sowohl mit dem Ursprungsland, der Türkei, als auch mit Deutschland.

$\mathrm{Da}$ das jeweilige Selbstkonzept von Individuuen allerdings komplex ist, wäre es nicht ausreichend, diese Menschen auf ihre Staatsbürgerschaft im Ankunftsland oder auf ihren türkischen Hintergrund zu beschränken oder jeweils nur einer Nation zu zuordnen (vgl. KEIM 2002). Die Kompexität der (Selbst)Verortung von Migranten zwischen den Nationen enthält positive und negative Aspekte. Obwohl die Migranten nach BOHNER et al. (2008) flexibler sind, sich variierenden sozialen Kontexten anzupassen und sich einzufügen, tritt bei Migranten mit multipler nationaler Identität eine Ambivalenz auf, die als stetiger Gradwandel zwischen der einen und der anderen Nation empfunden werden kann. Man könnte gemeinsprachlich tatsächlich von der Metapher des: 'Zwischen den Stühlen (der Nationen) Sitzens' sprechen. Dennoch haben diese Migranten, gerade wenn es sich um Minoritäten handelt, die Chance, ein Identifikationsangebot anzunehmen, das einer übergeordneten Identität entspringt und laufen dabei nicht Gefahr, ihre Distinktheit zu verlieren. Dennoch bleibt das Bild der zwischen den Stühlen sitzenden Migranten verhaftet, und sie können förmlich als Grenzgänger betrachtet werden, da sie weder voll zu der einen Nationalen Identität noch zu der anderen gehören.

HUDDY UND KHATIB (2007) konnten belegen, dass sich bei jungen, neuen Immigranten in den USA schnell eine nationale Identität herausbildet, und dass diese Identitätsbildung sich wiederum förderlich auf andere Formen der nationalen Bindung darstellt. Dem entgegen steht eine Untersuchung von VEDDER et al. (2007), der gemäß nur eine wenig ausgebildete bikulturelle Oreintierung bei Türkinnen und Türken in Deutschland nachgewiesen werden konnte.

Dieses Ergebnis erklären VEDDER et al. (2007) über die Wirkung des lang anhaltenden Rückkehr-Mythos, also der beharrlichen Erzählung, dass in unbestimmter Zukunft die Rückkehr in die alte Heimat erfolgen soll. Dieser Mythos ist nach VEDDER et al. dafür verantwortlich, dass ethnische Identität und Muttersprache erhalten bleiben und sich eine Separationshaltung einstellt. 
Weiterhin kommen der bislang schwierige Erwerb der deutschen Staatsbürgerschaft, sowie eine zunehmend ablehnende Haltung in den westlichen Gesellschaften gegenüber Immigranten und insbesondere gegenüber Muslimen zum Tragen und stärken ebenso das Rückbeziehen auf die Kultur des Herkunftslandes.

Diese negative Haltung konnte empirische in einer Untersuchung deutscher Probanden durch WESTLE (1999) bestätigt werden, in der sich gerade die ethnisch-kulturelle Konvergenz als bedeutsamer Erklärungsfaktor in der Vorhersage der Haltung der Probanden gegenüber Immigranten herausstellte.

VERKUYTEN (2005) hält fest, dass sich diese Diskriminierung, wahrgenommen als Bedrohung der Identität der Migranten, in einer erhöhten Identifizierung mit der Ingroup und einer Distanzierung zur gesellschaftlichen Mehrheit manifestiert, einem Ergebnis, welches mit den Vorhersagen der Social Identity Theory (SIT, nach TAJFEL UNDTURNER 1979) konform ist.

Auch die Forschung von Mummendey UND WALDZUS (2004) kommt zu ähnlichen Erkenntnissen, insofern als sie feststellt, dass ein niedriger sozialer Status in der deutschen Bevölkerung die Identifikation mit dieser für Individuen mit türkischstämmiger Migrationsgeschichte erschwert, wohingegen die Identifikation als Deutsch-Türken oder Türken eine distanzierende oder evasive Strategie darstellen kann.

Dass eine solche Abgrenzung von der Bevölkerung des Aufnahmelandes zu einer Disidentifikation mit diesem führen kann und sich diese nicht nur als Fehlen einer Identifikation mit der Aufnahmegesellschaft, sondern sogar in der Form einer reaktiven oder oppositionellen Identität niederschlagen kann, haben VERKUYTEN UND YILDIZ (2007) an Hand einer türkisch-niederländischen Stichprobe empirisch nachweisen können.

Man könnte daher ableiten, dass sich eine wenig ausgebildete nationale Identität in Verbindung mit einer stark augeprägten Disidentifikation nicht nur negativ auf die Wahrscheinlichkeit der gesellschaftlichen Partizipation auswirken, 
sondern dass eine solche Kombination sogar eine Gefährdung für die gesellschaftliche Stabilität und Kohäsion darstellen kann. Gestützt wird diese Schlußfolgerung von OGBU (2000), sowie im Hinblick auf den Zusammenhang zwischen nationaler Identität und politischer Partizipation auch von HUDDY UND KHATIB (2007).

Mittels des Modells zur Akkulturation nach BERRY $(1974,1980)$ können Zusammenhänge zwischen der Persönlichkeitsausgestaltung und der Anpassung von Migranten und deren Nachkommen erklärt werden; mittels der Erweiterung dieses Modells, wie sie das interaktive Akkulturationsmodell nach BOURHIS, MoISE, PERREAUlt Und SENÉCAL (1997) darstellt, lassen sich zusätzlich noch Einflüsse vonseiten der Aufnahmegesellschaft auf diesen Zusammenhang einbringen.

Aus eben diesem Grund sollen diese beiden Modelle im Folgenden beschrieben werden. Doch bevor der Begriff der Akkulturation im Verlauf dieser Arbeit thematisiert wird, soll zunächst ein weiterer Aspekt der kulturellen Identität betrachtet werden, nämlich die Komponente der 'Fremde':

\footnotetext{
Wer auf solche Unterschiede das Augenmerk richtet, folgert häufig: Die Migranten haben, man sieht es ja deutlich, die Sitten und Normen der Heimatländer bewahrt. Doch ein solches Urteil ist vorschnell, ist irreführend, ja falsch. Denn was dabei allein ins Blickfeld gerät, sind die für den deutschen Betrachter auffälligen Momente, die die Kontinuität des 'Fremden' und 'Anderen' repräsentieren. Systematisch ausgeblendet bleibt aber die andere Seite, eben jene, welche den deutschen Normalbetrachter nicht irritiert, nicht stört, nicht aufmerken läßt. Unsichtbar bleibt, mit anderen Worten, was die Migranten an Anpassungsleistungen tagtäglich erbringen und wie viele sich bemühen, die Anforderungen der neuen Umwelt mit ihren Gewohnheiten und Erwartungen zusammenzubringen; wie sie oft Elemente der einen mit Elementen des anderen zu verknüpfen versuchen, dabei ihre eigenen Formen des 'Kulturmix' entwerfen, vielerlei Zwischenformen, Mischformen, Übergangsformen. (BECK-GERNHEIM 2004: 41)
}

Die Betrachtung der Divergenzen beinhaltet die Gefahr, die Wahrnehmung der Ressourcen dieser Menschen und auch die Anstrengung, die sie zur Aktivierung dieser brauchen, völlig auszublenden. Die 'fremde' Kultur liefert somit auch Ressourcen, die Veränderungen bewirken können und die aus einer ethnozentrischen Perspektive nicht immer wahrgenommen werden können. Kulturelle Differenzen hindern nicht die Persönlichkeitsbildung, und auch die Annahme, dass kulturelle Unterschiede und widersprechende Persönlichkeitsentwicklung die Identitätsbildung behindern, ist nicht haltbar. So erklärt AUERNHEIMER: 
Es ist ziemlich sicher, dass normative Konflikte nicht notwendig zu einer 'Identitätdiffusion' führen müssen. Das Erlebnis der Diskrepanz von Werten, Normen etc. mag sogar unter günstigen sozialen Bedingungen der Persönlichkeitsentwicklung förderlich sein, weil es zur Reflexion anregt und zur Stellungnahme herausfordert. (AUERNHEIMER 1988: 124)

Obwohl eine "extreme“, kulturgeprägte Identität die

Persönlichkeitsentwicklung/-bildung ungünstig beeinflussen kann, beinhaltet sie auch ausreichend Potentiale und Ressourcen, woraus sich Kulturen entwickeln können. Kulturelle Identität kann jedoch nur entstehen, wenn Identitfikationsangebote vorhanden sind, und nicht im Lauf der Zeit - da beispielsweise nur mündlich tradiert - in Vergessenheit geraten. Um Kultur zu erinnern haben sich bereits frühe antike Kulturen ein kulturelles Gedächtnis geschaffen, dessen Konzeption im 21. Jahrhundert weiterhin relevant ist:

Gegen die Relativierung der Erinnerung, gegen die These, Erinnerungen bestünden unabhängig von der Vergangenheit, wie sie die Neurowissenschaften favorisiert, sprechen allein schon die psychischen Verletzungen, die sich oft erst nach Jahren Ausdruck verschaffen können. Selbst wenn eine tiefe Kluft besteht zwischen dem Ursprung einer Erfahrung und ihrer erinnerten Repräsentation, kommen Gemeinschaften nicht umhin, Fakten vorauszusetzen. Das schließt freilich die legitime Auseinandersetzung über den Status der Erinnerungen und ihren Gegenwartsbezug nicht aus. Die Probleme, die sich aus der gemeinschaftlichen Arbeit an der Vergangenheit ergeben, sind Gegenstand verschiedener Bemühungen um das kulturelle Gedächtnis. [...]

Solche Strukturen des [gesellschaftlichen] Zusammenhalts, von Assmann "konnektiv“ genannt, erfordern eine Engführung des Verhältnisses von Gedächtnis und Geschichte. Hier hatte Halbwachs nur einen Teil des Problems behandelt, nämlich das, was de Gruppen an Erinnerungen in den Einzelnen hineintragen. Diesen Aspekt der Abhängigkeit von den Milieus bezeichnet Assmann jetzt als das kommunikative Gedächtnis (J. Assmann 1992, 50). Wie bei Halbwachs sind die regelmäßige Interaktion, gemeinsame Lebensformen, lebendige Vergangenheit und ein aktiver Erfahrungsschatz einer Generation gemeint. Lebende, verkörperte Erinnerung, wie sie die „oral history“, die aus Zeugenaussagen hergestellte Geschichtsschreibung erkundet, umfasst nicht mehr als eine Periode von zwei bis drei Generationen, auf die sich diese Erinnerungen beziehen können. Selbst in Schriftkulturen haben die Menschen keine authentischen Erinnerungen, die weiter zurückreichen. Hinter ihren Wert- und Erfahrungshorizont kann keine Generation zurück. Er wandert mit dem fortschreitenden Blick der Gegenwart und verschwindet mit seiner Generation, mit der auch ihre alltagsnahen Erfahrungen schließlich untergehen.

(FAUSER 2008: 125f.)

Lebendige Vergangenheit zu praktizieren, spürbare Geschichte zu erinnern und somit die kulturelle Geschichte zu verkörpern, schafft bei den Erinnerungsträgern eine Bindung zueinander, zu der Kultur, zu der Erinnerung und führt somit zu einer kulturellen Identität und zu einem kollektiven Gedächtnis, welches das Vergessen verhindert und damit die Identität aufrecht erhält:

Die zahllosen symbolischen Inszenierungen des Kollektivgedächtnisses dienen der stetig wiederholten Bekräftigung von Vereinbarungen und schaffen so eine Struktur der Bindung, die mittels vergangener und nicht authentischer Erfahrungen die Zusammengehörigkeit beschwört [Hervorhebung I.Ö.]. Verlorene oder verloren geglaubte Beziehungen möchte 
man wiederherstellen. Was keine Gegenwart mehr hat, was nicht mehr weiterwirkt, wird entgegen dieser fehlenden Gegenwart verstärkt erinnert und dem Alltag auf symbolischem Wege wieder hinzugefügt. Dieser „kontrapräsentische“ (ASSMANN 1992, 78ff.) Zug verleiht den kollektiven Anstrengungen die orientierende Kraft. Allerdings wirkt hier eine Transformation: „Durch Erinnerung wird Geschichte zum Mythos.“ (ASSMANN1992, 52). $[\ldots]$

Das kulturelle Gedächtnis ist eine mediale Steigerungsform des kollektiven Gedächtnisses. Aber auch diese Modifikation bewirkt eine Transformation. Sie geht nicht nur in einer temporalen Verlängerung auf, sondern schafft einen qualitativ anderen Bezug zur Vergangenheit. Indem sich das kulturelle Gedächtnis ganz auf eine mediale Überlieferung stützt, deren Weitergabe auf Bildungsinstitutionen angewiesen ist, führt es alleine aufgrund seiner materialen Beschaffenheit eine Art Eigenleben. Zwar erlaubt es „eine freie Verfügung des Einzelnen über die Erinnerungsbestände" (ASSMANN 2000, 34) und scheint die Chance der selbstverantworteten Orientierung zu gewähren. Aber auf der anderen Seite entspricht das kulturelle Gedächtnis genau dem typisch modernen Verlust an Verbindlichkeit und befördert Vergessen. Anders gesagt: gerade weil sich das kulturelle Gedächtnis auf die Archive stützt, ist das, was es produziert, nur noch eine Sache der Bildung:

Das kulturelle Gedächtnis umfasst im Gegensatz zum kommunikativen Gedächtnis das Uralte, Abgelegene, Ausgelagerte und im Gegensatz zum kollektiven und Bindungsgedächtnis das Nichtinstumentalisierbare, Häretische, Subversive, Abgespaltene. Mit dem Begriff des kulturellen Gedächtnisses ist die äußerste Entfernung von dem erreicht, was unseren Ausgangspunkt gebildet hat: das individuelle Gedächtsnis in seinen neuronalen und sozialen Bedingungen. (ASSMANN 2000, 41) [...]

Besonders wichtig ist ihr [der Gedächtnisgeschichte - Anm. I.Ö.] der dynamische Austausch zwischen Speicherformen und gelebter Kommunikation, also der Prozess der Wiedereinführung ausgeschiedenen Wissens ins kollektive Gedächtnis. Indem sie nun auch noch den modernen Interpreten in das Geschehen mit einbezieht, erreicht die Theorie die Ebene der Kulturbedeutung von Traditionen, womit sie sämtlichen symbolischen Formen des Rückbezugs ein methodisches Gerüst anbieten kann. Die Theorie betont den hohen Anteil des Imaginären an der Geschichte der Zivilisation und sie nimmt die Realität schaffenden Komponenten der mentalen Umdeutungen des Geschichtlichen ernst (Harth 1998, 105). Das ist vielleicht ihr größter Gewinn. (FAUSER 2008: 125-129)

Die kollektive Identität entwickelt somit durch eine Rückzugstendenz in das kulturelle Gedächtnis bestimmte Bindungen, welche wiederum auch das kollektive Gedächtnis steigern:

Die kollektive Erinnerung oder das offizielle Gedächtnis, in dem sich auch Vorurteile ohne Rücksicht auf ihre wissenschaftliche Widerlegung wirksam bleiben, schafft an der gemeinsamen Identität, mit der sie breit akzeptierte Gründungsereignisse entwirft.

[...]

Gegen die vorschnelle Kritik am Begriff der kollektiven Identität, die eine Selbstreflexion der Terminologie fordert (L. NIETHAMMER 2000) kann man daran erinnern, dass der Ort des Handelns innerhalb der diskursiven Formationen bestimmbar sein sollte und auch Formen der gemeinsamen „agency“ bestehen, die der Begriff erfassen will. Außerdem sind dem wissenschaftlichen Zugriff sowieso nur Teilidentitäten zugänglich, die in Wechselwirkung mit anderen stehen. Hier muss man die Partialität des Themas unterstreichen. So wie man "das" kollektive Gedächtnis als Ganzes nie umgreifen kann, werden auch nicht alle Seiten des Themas de sozialen Verankerung von Identitäten zugänglich sein. Bestes Beispiel: die Identitätskonstruktion in Deutschland nach dem zweiten Weltkrieg. Sie bestand wesentlich im öffentlichen Eingeständnis der Schuld, im Bekenntnis der Nation zur Verantwortung für die jüngste Vergangenheit. Eine solche „universalistische Codierung von kollektiver Identität“ (GIESEN 1999, 67f.) kann sich nur auf ein funktionierendes kollektives Gedächtnis als Instrument der Gemeinschaftsbildung 
gründen. Gleichzeitig deckt es aber nur wiedereinen Aspekt ab, wie der Wandel des Umgangs mit der Vergangenheit und die Annäherung der Erinnerungskulturen in Ost und West zeigten.

Der umstrittene Begriff benennt also eine Form sozialer Beziehungen, die symbolisches Handeln mit einem expliziten Selbstbezug verknüpft:

Wie eine individuelle Biographie bestimmte Phasen der Selbstfindung und Identitätsformation kennt, so finden sich auch in der Geschichte sozialer Beziehungsnetze besondere Perioden und Räume, in denen die Kommunikation sich auf die Bestimmung und Neubestimmung der Außengrenzen und des kollektiven Selbst richtet. (GIESEN 1999, 23)

Hinzufügen muss man nur die Bedeutung des kollektiven, aber wohl auch des kulturellen Gedächtnisses, die dann ebenfalls thematisiert werden müssen und aus dem Prozess selber verändert hervorgehen. (FAUSER 2008:133f)

Die individuelle kulturelle Identität ist somit nicht erfassbar, weil sie Anteile von kollektivem und kulturellem Gedächtnis enthält. Dies wird im individuellen Handeln auf symbolischer Ebene dargestellt. Ein weiterer Aspekt der kulturellen Identiät, und mit diesem soll dieser Kurzabriss schließen, sind weitere Werte und Beziehungen, z.B. besonders in türkischstämmigen Familie die Themen Autorität und die Selbstverständlichkeit, ältere Familienangehörige auf verschiedenen Wegen zu unterstützen:

So ist zum Beispiel das Familienleben der Deutschen vor allem an der Kernfamilie ausgerichtet, die Beziehungen zwischen Mann und Frau, Eltern und Kindern stehen im Zentrum; und in diesem Beziehungsgefüge sind persönliches Glück, Partnerschaft, Selbstbestimmung und Selbstständigkeit vorrangige Werte. Bei den Migranten dagegen hat der weitere Familienverband mehr Gewicht, Autorität und Seniorität spielen eine größere Rolle, auch die Erwartung bzw. Verpflichtung zu wechselseitiger Unterstützung ist stärker ausgeprägt. (BECK-GERNHEIM 2004: 40)

Im Vergleich zu den im bundesdeutschen Durchschnitt eher vertretenen Kleinfamilien spielt die Wertigkeit der Großfamilie für Menschen mit Zuwanderungsgeschichte eine große, Identitätsstiftende Rolle - und das schon seit Beginn der Einwanderungsgeschichte türkischer Migranten in Deutschland. 


\subsection{Migrationsgeschichte}

Einwanderer definiert WoLLENSCHLÄGER (1994) als „Personen, die - im Gegensatz zu Flüchtlingen bzw. Asylsuchenden - aus freien Stücken in die Bundesrepublik einreisen, um hier auf Dauer ihren Lebensmittelpunkt zu finden“. Dabei merkt er im gleichen Zug an, dass diese Definition auf den Großteil der 1955-1973 angeworbenen Gastarbeiter anfangs bezüglich der dauerhaften Bleibeabsicht nicht zutraf. Diese Einschränkung kann als durch die politischen Bedingungen der aktiven Anwerbezeit in Aufnahme- und Entsendeland bedingt angesehen werden. Die seit Beginn der Anwerbepolitik Mitte der 1950er Jahre bis in die heutige Zeit vollzogene Entwicklung von Migration und Integration in Deutschland soll im Folgenden beleuchtet werden.

Seit Ende des Zweiten Weltkrieges ist die Anzahl der in der Bundesrepublik Deutschland lebenden Ausländer stetig gestiegen. Ende des Jahres 2008 lebten rund 6,7 Millionen Ausländer (StATISTISCHES BundesAMT, 2009), davon 1,7 Millionen Personen türkischer Abstammung (BAMF, 2010), in Deutschland. Sie lassen sich in vier große Zuwanderungsgruppen aufteilen: 1. Flüchtlinge und Vertriebene als Folge von Kriegen, 2. angeworbene „Gastarbeiter" und deren Familien, 3. Flüchtlinge und Asylsuchende, 4. „Aussiedler“ bzw. „Spätaussiedler“ (BADE, 1997; BommES, 1997). Dieses Kapitel soll sich v.a. der Geschichte von Migration im Sinne der zweiten Gruppe, der „Gastarbeiter“, widmen.

In der Bundesrepublik wurden zwischen 1955 und 1973 von Unternehmen und Behörden Millionen ausländischer Arbeitskräfte (so genannte "Gastarbeiter") aus verschiedenen Mittelmeerländern angeworben (BOMMES, 1997; BUNDESAMT FÜR MigRATION UND FLÜCHTLINGE, 2010). Sie und ihre Familien bilden bis heute die größte Gruppe der in Deutschland lebenden Menschen mit Migrationshintergrund (BUNDESZENTRALE FÜR POLITISCHE BILDUNG, 2005).

Der wirtschaftliche Aufschwung der 50er Jahre brachte einen erhöhten Bedarf an Arbeitskräften mit sich (BAMF, 2005; BOMMES, 1997). Die zahlreichen Arbeitsplätze konnten jedoch durch die im Krieg reduzierte Anzahl erwerbsfähiger Männer nicht gedeckt werden; eine Erwerbstätigkeit der Frau galt als unüblich (MATTES, 2005; YANO, 1998). Besonders die Landwirtschaft und das Hotel- und Gaststättengewerbe beklagten einen Kräftemangel und den Unwillen deutscher Arbeitssuchenden, in diesem Gewerbe zu arbeiten: „Freiwillig war damals offensichtlich kaum ein Arbeitsloser bereit, zu den kargen Lohnbedingungen in der 
Landwirtschaft zu arbeiten. Unregelmäßige Arbeitszeiten, Arbeit bei Wind und Wetter und oft an Sonn- und Feiertagen schreckten die meisten ab." (MEIERBRAUN, 1995: 18). Auf Druck besonders betroffener Wirtschaftsbereiche ging die deutsche Regierung auf vorherige Anfragen der italienischen Regierung ein und unterzeichnete nach einer Verhandlungsphase am 20. Dezember 1955 das deutsch-italienische Anwerbeabkommen (BOMmES. 1997; D'AMATO, 2005; SONNENBERGER, 2003). Es folgten weitere „Anwerbeverträge“ mit Spanien und Griechenland (1960), mit der Türkei (1961), Portugal (1964), Tunesien, Marokko (1965) und Jugoslawien (1968) (BADE, 1994; BAMF, 2010; KURTOĞLU, 2008, STEINERT, 1995). Dabei ging die Initiative jeweils stets von den Anwerbeländern aus. $^{15}$

Die Bewerber mussten sowohl für die jeweilige Beschäftigung („Arbeitsverwendungsfähigkeit“), als auch für den Aufenthalt in der BRD („aus seuchenhygienischen Gründen“) ${ }^{16}$ gesundheitlich geeignet sein. Dies wurde im Rahmen einer ärztlichen Untersuchung, die in Gruppen mehrerer Bewerber absolviert wurde, festgestellt (SCHIRRMACHER, 2006; YANO, 1998). Zudem durften die Bewerber nicht die Altersgrenzen überschreiten, vorbestraft, unordentlich gekleidet, Analphabet oder drogenabhängig sein (ERYILMAZ, 1998). Qualifikationen durch Schul- oder Berufsausbildung waren zweitrangig (SCHIRRMACHER, 2006).

Viele der angeworbenen Arbeitskräfte stammten aus strukturschwachen Gegenden ihres Ursprungslandes und verfügten größtenteils über einen niedrigen Bildungsstand (KURTOĞLU, 2008). Sie arbeiteten in Deutschland meist als un- oder angelernte Arbeiter, im weiteren Verlauf der Anwerbephase überwiegend im produzierenden Gewerbe und im Baubereich (BOMMES, 1997; Boos-NüNNING, 1998). Trotz der Tendenz der jüngeren Generationen, einen höheren Schulabschluss zu erreichen, hat sich dieses Bildungsdefizit im Vergleich zu anderen Bevölkerungsgruppen erhalten (BAMF, 2010; HUNGER UND THRÄNHARDT, 2004; Gogolin, 2000).

Langfristige Konzepte einer "Gastarbeiterpolitik“ existierten zu der Zeit nicht. Der umgangssprachliche, nie amtlich bestätigte Begriff des „Gastarbeiters“ spiegelt ebenfalls die öffentliche Meinung wider, dass die Beschäftigung von

15 Für die türkische Regierung standen dabei drei Nutzen im Vordergrund: die Entlastung des eigenen Arbeitsmarktes, der Transfer von Devisen in das eigene Land und die Gewinnung von qualifizierten, zurückgekehrten Arbeitnehmern (YANO, 1998).

16 Diese Bedingung war nur Teil des Anwerbeabkommens mit der Türkei und Tunesien, nicht aber mit den anderen, innereuropäischen Abkommen (YANO, 1998). 
ausländischen Arbeitsnehmern keine Dauererscheinung darstelle (BADE, 1994). Es wurde angenommen, dass die angeworbenen Arbeitskräfte kurz- und mittelfristig wieder in ihre Heimatländer zurückkehren würden (BADE, 1997). Aus diesem Grund wurden die sozialen Folgen von längeren Arbeitsaufenthalten wenig berücksichtigt. Die sozialen und kulturellen Bedürfnisse der Angeworbenen wurden kaum beachtet (YANO, 1998). „Ausländerpolitik war [...] kaum mehr als Arbeitsmarktpolitik, angewendet auf Ausländer." (BADE, 1994: 53). Das zunächst angewandte Rotationsprinzip (Aufenthaltsbeschränkung auf zwei Jahre), welches 1964 aufgrund der immer wieder neuen und kostspieligen Einarbeitungszeiten in den Betrieben aufgegeben wurde, sollte eine langfristige Niederlassung verhindern (BADE, 1997; JAMIN, 1998; KURTOĞLU, 2008; YANO, 1998). Die Unterbringung in Barackenunterkünften (BADE, 1997) spiegelt das damalige Leben in einem Provisorium wider. ${ }^{17}$

Zwar waren die Arbeitgeber verpflichtet, für eine angemessene Unterkunft zu sorgen, aber in der Praxis schienen die Arbeitsämter den Zustand der Sammelunterkünfte kaum kontrolliert zu haben (YANO, 1998; ERYILMAZ, 1998a). Eine gemischte Unterbringung von deutschen und ausländischen Arbeitnehmern wurde von der Bundesanstalt für Arbeitsvermittlung und Arbeitslosenversicherung abgelehnt (YANO, 1998). Auch ein Familiennachzug wurde von der deutschen Politik nicht angestrebt: „Das Nachziehen von Familienangehörigen türkischer Arbeitnehmer - insbesondere von Frauen mit mehren Kindern - liegt zweifellos nicht im deutschen Interesse." (Regierung von Oberbayern an das Bayrische Staatsministerium des Innern vom 26.11.1964, zit. nach MATTES, 2005: 153). Ein Fehlen an nötiger Infrastruktur (z.B. Wohnraum, Bildung) für Zuwandererfamilien erscheint vor diesem Hintergrund kaum verwunderlich (Boos-NüNNING, 1994).

Bis in die Mitte der 1970er hinein gestaltete sich die Politik des Wohlfahrtsstaates Deutschland unterscheidend gegenüber In- und Ausländern: Zugunsten einer leichten Regulation des Arbeitsmarktes wurden ausländische Arbeitnehmer den inländischen gegenüber benachteiligt. Zwar galt für sie gleiches

17 KURTOĞLU (2008) beschreibt diese Wohnsituation: „Viele lebten in einem Zimmer mindestens zu zweit oder mit mehreren zusammen. Kochen mussten sie entweder im gleichen Wohn- bzw. Schlafraum oder in einer kleinen Gemeinschaftsküche, die in der Regel relativ dürftig ausgestattet war. Ein Ausruhen war kaum möglich. Zu diesen Wohnverhältnissen kamen die Störungen, die durch die Schichtarbeit entstanden. Bei solchen primitiven Zuständen war an gemütliche Stunden oder an den Empfang privaten Besuches kaum zu denken." [...] PAZARKAYA (1989) schreibt „In manchen Heimen hingen sogar Regeln zur Hausordnung an der Wand: Wo der Aschenbecher stehen soll, wann man Hausschuhe tragen und wie man sich auf das Klo setzen soll." (2008: 104). 
Tarif- und Arbeitsrecht, jedoch wurden sie durch das Inländerprimat bei der Vergabe von Arbeitsplätzen, sowie durch einen großen Spielraum der Arbeitsämter bei der Vergabe bzw. dem Entzug der Arbeitserlaubnis mit der Folge eingeschränkter Arbeitslosengeldzahlungen systematisch benachteiligt. Dieser Willkürvorbehalt wurde erst 1977 aufgegeben (BOMMES, 1997).

Eine Rückkehr in das Heimatland lag anfangs auch im Interesse der angeworbenen Arbeitskräfte (SCHIRRMACHER, 2006). Zumeist war ihr Aufenthalt mit dem Zweck verbunden, im Aufnahmeland Geld zu verdienen, welches sie zu ihren Familien in die Heimat transferierten. Ihr Leben in Deutschland, außerhalb des Familienverbandes, zeichnete sich durch Konsumverzicht und die durch spärliche Gemeinschaftsunterkünfte geprägte Wohnkultur aus (BADE, 1994). Auch nach Zuzug der Familien blieb der „Rückkehr-Mythos“ noch lange erhalten (KESKIN, 1998), welcher sich im Erhalt einer starken ethnischen Identität, einer Separationseinstellung und guter türkischer Sprachkenntnisse der folgenden Generation äußerte (VEDDER, SAM UND LIEBKIND, 2007).

Anfang der 1970er wurde dann eine zunehmende Verlagerung des Lebensmittelpunktes nach Deutschland durch ausländische Arbeitnehmer und die öffentliche Diskussion der „Gastarbeiterfrage“ immer deutlicher. Die Lebensweisen und -umstände bzgl. Konsumverhalten, Bevölkerungsstruktur, Siedlungsweise, Aufenthaltsdauer und Bleibeabsicht der ausländischen Arbeitnehmer hatten sich von denen eines alleinlebenden „Gastarbeiters“ zu denen einer in Deutschland niedergelassenen Familie gewandelt (BADE, 1994; BOMMES, 1997, KESKIN, 1998).

Entstandene „Einwandererkolonien“, vielfach als Abkapselung gewertet, sind als natürliches Phänomen des Einwanderungsprozesses und „Kulturschleuse zwischen alter und neuer Welt“ (BADE, 1997) zu betrachten. Sogenannte Parallelgesellschaften, von der inländischen Bevölkerung als Bedrohung oder Scheitern der Integration wahrgenommen, dienen den Migranten nach ihrer Ankunft als „Halt in der Fremde“ (FUHRKEN, 2008). Unter der Prämisse der Freiwilligkeit und Offenheit nach Außen erachtet sie OBERNDÖRFER (2001) als legitim. Zudem zeichneten sich die angeworbenen Arbeitsnehmer bei ihrer Ankunft eigentlich durch den Wunsch nach Kontakt zur deutschen Bevölkerung aus. Vor allem die isolierte Unterbringung begünstigte eine „Notgemeinschaft in Subkultur“ und einen intensivierenden Diaspora-Effekt auf die Religiosität (ERYILMAZ, 1998a; YANO, 1998). 
Doch nicht nur zwischen den Gastarbeiterfamilien und der deutschen Gesellschaft, sondern auch innerhalb der Familien entstanden Spannungen: Viele Familien waren mit einem Kulturkonflikt konfrontiert zwischen den selbst immigrierten Eltern und den in Deutschland aufgewachsenen und geprägten Kindern der zweiten Einwanderungsgeneration (BADE, 1994).

Im Zeichen des steigenden Ölpreises und der Wirtschaftsrezession endete die Anwerbephase mit dem Anwerbestopp im November 1973. Zu diesem Zeitpunkt lebten von den zwischen 1955 und 1973 eingewanderten 14 Mio. Ausländern noch ca. 3 Mio. im Bundesgebiet, davon ca. 15\% Italiener und 30\% Türken (BADE, 1994, 1997; BAMF, 2005; BOMMES, 1997; KURTOĞLU, 2008). Der natürliche Niederlassungsprozess wurde durch die jetzt entfallende Wiederkehroption nach einer Rückkehr in das Heimatland noch verstärkt. Besonders die Rückkehr in die Türkei erschien im Gegensatz zu z.B. Griechenland und Spanien wegen der schwierigen politischen und ökonomischen Situation wenig attraktiv (BOMMES, 1997). Für türkische Bürger waren nun die Familienzusammenführung, d.h. der Nachzug der Ehefrauen und Kindern von Gastarbeitern der ersten Einwanderungsgeneration oder die Eheschließung mit einem in Deutschland lebenden Türken ${ }^{18}$ die einzigen Möglichkeiten, eine Aufenthalts- und Arbeitserlaubnis für Deutschland zu bekommen (KURTOĞLU, 2008).

Trotz Versuchen der Förderung der Rückkehrbereitschaft sank die Anzahl der in Deutschland lebenden Ausländer nach Beendigung des aktiven Anwerbens nicht, sondern stieg weiter an, während die Erwerbsquote durch den Nachzug von Familienmitgliedern sank (BADE, 1994, 1997; BOMMES, 1997; JAMIN, 1998; KURTOĞLU, 2008). Der Frauenanteil, sowie der von Kindern und Jugendlichen in der Migrantenbevölkerung stiegen kontinuierlich an (BOMMES, 1997; KALTER UND GRANATO, 2004). Durch Anwerbestopp und Familiennachzug setzte ein demographischer Wandel ein. Die Notwendigkeit einer angemessenen Integration der Kinder und Jugendlichen in Schule und Ausbildung führte letztendlich zur Aufgabe des Willkürvorbehaltes und somit zum Ende der rechtlichen Benachteiligung von Gastarbeiterfamilien gegenüber Deutschen. Dies sollte den ausländischen Familien eine Absicherung ihres Aufenthaltes und eine Lebensplanung in Deutschland ermöglichen. Ebenso wurden spezifische

18 Im Schnitt kann man davon sprechen, dass ca. 16.000 „Import“-Bräute und -Bräutigame pro Jahr aus der Türkei nach Deutschland kamen. 
Bildungsangebote für nachwandernde ausländische Jugendliche geschaffen, welche innen den Einstieg in Bildung und Arbeitsmarkt erleichtern sollten (BOMMES, 1997).

Anfang der 1990er stellten türkischstämmige Menschen mit Migrationshintergrund die am stärksten angewachsene Gruppe: knapp ein Drittel aller in Deutschland lebender Ausländer, davon viele mit langen Aufenthaltszeiten (Bade, 1994). 35\% der seit 1961 eingewanderten Türken waren zu diesem Zeitpunkt in ihr Heimatland zurückgekehrt (MARSCHALCK, 1997). Ende der 1980er besaß nur noch ein geringer Anteil (11\%) der in Deutschland Verbliebenden konkrete Rückkehrpläne (BADE, 1994). Schon lange war „in Deutschland lebende Türken" keine ausreichende Beschreibung mehr: Ihr Lebensmittelpunkt war in Deutschland, die Kinder der damals selbst Eingewanderten wuchsen in der deutschen Gesellschaft auf und in der Türkei galten sie nicht mehr als „Türken“, sondern wurden als „Deutschländer“ bezeichnet (BADE, 1994). Sie waren „längst nicht mehr Fremde mit deutscher Aufenthaltsgenehmigung, sondern Deutsche mit einem fremden Pass" (BADE, 1994, S.50) und ein Großteil der jungen Türken zeigte die Absicht, in Deutschland zu bleiben (BAMF, 2010) sowie Interesse an der deutschen Staatsbürgerschaft (BADE, 1994). ${ }^{19}$

Dieser Trend zur endgültigen Niederlassung schlug sich allerdings wenig in den Beschäftigungsverhältnissen wieder. So gelang zwar einigen der soziale Aufstieg - es bildete sich eine differenzierte Sozialstruktur mit einer schmalen Oberschicht, einem wachsenden Mittelstand und einer breiten Arbeiterbevölkerung-, die Mehrheit jedoch arbeitete weiterhin unter harten und gefährlichen Arbeitsbedingungen bei geringem Lohn, in Jobs, die unter deutschen Arbeitnehmern wenig beliebt waren (BADE, 1994; BAMF, 2010; BOMMES, 1997; Boos-NüNNING, 1998). Die Anzahl der Kinder aus Migrantenfamilien mit Abitur oder Hochschulabschluss war zwar gestiegen (von 1.8\% 1970 auf 5.5\% 1996), lag dennoch immer noch weit unter dem Niveau deutscher Kinder. Türkische Migranten wiesen zu diesem Zeitpunkt hinsichtlich ihrer Bildungsverteilung die größte Abweichung zur Vergleichsgruppe der Deutschen auf (KALTER UND GRANATO, 2004). Diese Unterrepräsentation türkischer Jugendlichen bei höheren

19 Allerdings fühlte sich diese Gruppe zunehmend mit Ausländerfeindlichkeit konfrontiert und favorisierte u.a. dadurch das Modell der Doppelten Staatsbürgerschaft - Ein Prozess der Stärkung einer Minoritätsidentität durch Ablehnung der wahrgenommenen Majorität, der durch sozialpsychologische Erkenntnisse (TAJFEL \& TURNER, 1979; SCHMITT \& BRANSCOMBE, 2002, zit. n. VERKUYTEN, 2005) gut erklärbar ist. 
Bildungsabschlüssen hat sich bis dato nicht wesentlich gebessert (KURTOĞLU, 2008; SCHIRRMACHER, 2006). Auch die Wohnverhältnisse spiegelten die geringe Integration der ausländischen Bevölkerung in deutsche Städte wider: eine Konzentration ausländischer Einwohner auf bestimmte Stadtteile überstieg die Anfang der 1970er angestrebten 12\% (BADE, 1994).

Eine andere Entwicklung zeigt die zunehmende, endgültige Niederlassung in Deutschland. Über vermehrte Betriebsgründungen schuf der türkischstämmige Mittelstand Arbeitsplätze (ein Drittel von Deutschen besetzt) und erwirtschaftete Kapital, welches wiederum nicht mehr in das Heimatland transferiert, sondern in Immobilien, Bausparverträge etc. in Deutschland investiert wurde (BADE, 1994; BOMmES, 1997; BOOS-NüNNING, 1998; KURTOĞLU, 2008; ŞEN, 1998).

Deutschland war somit faktisch ein „echtes“ Einwanderungsland geworden, was jedoch von der damaligen Regierung bis in die 1990er Jahre verneint wurde und sich in einer weiterhin arbeitsmarktorientierten Ausländerpolitik und fehlenden aktiv betriebenen Einwanderungspolitik widerspiegelte (BADE, 1994, 1997; BOMMES, 1997; WolLENSCHLÄGER, 1994). BADE (1996: 424) bezeichnet die 1980er Jahre diesbezüglich als "verlorenes Jahrzehnt“. Die (Nicht-) Integration von ausländischen Mitbürgern fand immer wieder Einzug in Wahlkampfthemen, wobei Parteien die wachsende Beunruhigung der Bevölkerung über soziale Folgen der Einwanderung für ihre Zwecke nutzten (BADE, 1994; WolLENSCHLÄGER, 1994). Eine konzeptlose, wenig langfristige Ausländerpolitik Anfang der 1980er verstärkte diese Beunruhigung zunehmend und zeigte sich Anfang der 1990er als teilweise verantwortlich für die beginnende „Politikverdrossenheit" sowie für vereinzelte, in der Öffentlichkeit aber stark wahrgenommene gewalttätige Übergriffe gegenüber Ausländern (BADE, 1994; WolLENSCHLÄGER, 1994). Ein neues Konzept der Ausländerpolitik war längst überfällig.

1990 verabschiedete die damalige Bundesregierung nach langer Auseinandersetzung das neue Ausländergesetz, welches zwar die Einbürgerung junger Ausländer vereinfachte, eine Assimilation jedoch zum Nachteil einer echten Integration förderte (BADE, 1994). So wurde (außer in wenigen Ausnahmen) eine doppelte Staatsbürgerschaft durch das Gesetz nicht ermöglicht und die ursprüngliche Staatsbürgerschaft musste bei Einbürgerung abgegeben werden. ${ }^{20}$

20 Die nun mögliche Einwanderung wurde nur zurückhaltend genutzt. Eine Aufgabe der ursprünglichen Staatsbürgerschaft erschien vor dem Hintergrund der bisherigen "Gastarbeiterpolitik“ (mit der Botschaft, nur kurz-, aber nicht langfristig in Deutschland 
Auch das kommunale Wahlrecht für schon lange in Deutschland Ansässige wurde Migrantinnen und Migranten weiterhin verwehrt (Bade, 1994; Wollenschläger, 1994). Die politische Teilhabe von Mitbürgern mit ausländischem Pass wurde durch das neue Ausländergesetz somit nicht weiter gefördert. Gegenteilig trug es zu einer defensiven, im Extremfall sich abgrenzenden Haltung bei (Bade, 1994). Besonders restriktiv gestaltete sich das Niederlassungsrecht gegenüber Mitbürgern aus Nicht-EG-Ländern (Wollenschläger, 1994), z.B. der Türkei.

Mit der Zeit wurde eine zunehmendes Problem immer deutlicher: Jungen Menschen der zweiten und dritten Einwanderungsgeneration war das Herkunftsland der Eltern vermehrt keine „Heimat“ und somit kein fester Bestandteil der eigenen Identität mehr. Wachsender Fremdenhass, geringer Schutz davor durch den deutschen Staat und weiterhin nicht ausreichende Integrationsansätze förderten ebenfalls nicht, Deutschland als die eigene Heimat anzunehmen. Ein Identitätskonflikt der fehlenden Zugehörigkeit gestaltete sich als prägend für die Generationen der nicht selbst zugewanderten Menschen mit Migrationshintergrund (BADE, 1994).

Im Zeichen der zunehmenden fremdenfeindlichen Übergriffe in den Jahren 1992 und 1993 wurde das ausländerpolitische Vorgehen erneut und diesmal umfassender in Gesellschaft und v.a. in der Politik diskutiert. Die Regierungspartei CDU/CSU beharrte allerdings hierbei im Gegensatz zu ihrem Koalitionspartner FDP auf einem konservativen, auf Einwanderungsbeschränkung fixierten Kurs. Einbürgerungserleichterung und doppelte Staatsbürgerschaft wurden weiterhin verwehrt (BADE, 1994) und damit auch die faktische Einwanderungssituation (BAMF, 2005; BPB, 2005a). Wissenschaftler mahnten die fehlende Gestaltung von Migration und deren Folgen an (BADE, 1994a). Bis auf kleinere Veränderungen im Rahmen der Novellierung des Ausländergesetzes 1996 (weitere Erleichterung von Einbürgerung und Familiennachzug) wurden die zentralen Probleme der Integration jedoch nicht gelöst (BPB, 2005a). Die Arbeitslosenquote in der ausländischen Bevölkerung blieb im Vergleich zur Gesamtbevölkerung deutlich erhöht und zeigte einen Zusammenhang $\mathrm{zu}$ fehlender Schul- und

willkommen zu sein) und des gegenwärtigen Fremdenhasses wenig attraktiv (BADE, 1994, 1997). Trotz hoher Identifikation mit Deutschland (65,2\% fühlen sich mit Deutschland verbunden), würden aktuell nur knapp 30\% zugunsten einer deutschen Staatsbürgerschaft ihre ursprüngliche aufgeben (BAMF, 2010). Besonders frustrierend für Ausländer mit langem Aufenthalt in Deutschland gestaltete sich das als inkonsequente erlebte Verfahren mit Aussiedlern aus Osteuropa, die problemlos beide Staatsbürgerschaften erhalten durften (BADE, 1994; ZAGEFKA \& BROWN, 2002; WOLLENSCHLÄGER, 1994). 
Berufsausbildung (Boos-NüNNING, 1998).

Erst Ende der 1990er gab es eine Wende in der deutschen Migrationspolitik (ANGenendt Und KRUSE, 2003; MEIER-Braun, 2002). Die Staatsangehörigkeitsrechtsreform wurde 1999 unter der Federführung von Bundesinnenminister Schily verabschiedet und trat am 01.01.2000 in Kraft. Die Vergabe der deutschen Staatsangehörigkeit folgte nun nicht mehr dem ethnonationalistisch geprägtem Abstammungsprinzip („ius sanguinis“), sondern dem Territorialprinzip („ius soli“). Die Geburt in der Bundesrepublik ermöglichte nun (eingeschränkt) den Erwerb der deutschen Staatsbürgerschaft. Ein Kind erhielt somit bei Geburt die deutsche Staatsbürgerschaft, wenn dessen Eltern bereits 8 Jahre in Deutschland lebten. Bei doppelter Staatsbürgerschaft musste sich das Kind vor dem 23. Lebensjahr für eine entscheiden (BPB, 2005a; BAMF, 2005, HAILBRONNER, 2001). Auch die Einbürgerung von selbst Zugewanderten war jetzt bereits nach 8 Jahren (vorher 15) möglich (HAILBRONNER, 2001) und wurde vermehrt genutzt. Die Anzahl der Deutschen mit türkischer Herkunft steigt seitdem stetig an. Der Begriff der „Bindestrichdeutschen“ (z.B. Deutsch-Türken) fand durch die neue Regelung der Staatsbürgerschaft verstärkt Einzug in den deutschen Sprachgebrauch (KURTOĞLU, 2008).

Im Zeichen der demographischen Entwicklungen der deutschen Gesellschaft wurden Motive der vergangenen Anwerbezeit wieder aktuell: der Bedarf an ausländischen Arbeitskräften. Die Initiative des damaligen Bundeskanzlers Schröder, hochqualifizierte Computerspezialisten mit Hilfe der "Green Card“ nach Deutschland zu holen (BAMF, 2005; BPB, 2005a), weist sowohl Gemeinsamkeiten, als auch Unterschiede zur Anwerbung von Gastarbeitern in den 1950ern und 1960ern. Der Bedarf an Arbeitskräften sollte durch angeworbene Arbeitnehmer gedeckt werden. Ein längerer Aufenthalt war erneut nicht vorgesehen. Jedoch fand diese Anwerbung nicht in den gleichen Ausmaßen wie in den Jahren zuvor statt. Zudem handelte es sich bei den GreenCard-Besitzern lediglich um hochqualifiziertes Personal. Benötigt wurde ihre Expertise und ihr Know-how, nicht wie damals ihre reine Arbeitskraft. KOLB (2004) bezeichnet die Green-Card-Initiative als „Elitemigration“. Trotz nur vorübergehender Arbeitserlaubnis im Rahmen der Green Card sollte durch umfassende Hilfen die Integration dieser Arbeitsmigranten gefördert werden.

Im weiteren Verlauf prägte die 2001 einberufene Kommission 
„Einwanderung“ unter dem Vorsitz von Rita Süßmuth die fachliche und politische Diskussion über die zukünftige Migrations- und Integrationspolitik. Die dort erarbeiteten Empfehlungen bildeten die Grundlage für den Entwurf des neuen Zuwanderungsgesetzes, welches zum 01.06.2002 in Kraft trat, wenige Monate später jedoch vom Bundesverfassungsgericht für ungültig erklärt wurde (BAMF, 2005; BPB, 2005a). Nach einem neuen Gesetzgebungsprozess wurde im Sommer 2004 eine überarbeitete Version des Zuwanderungsgesetzes verabschiedet, welches am 01.01.2005 in Kraft trat. Einreise- und Aufenthaltsbedingungen für hoch qualifizierte Zuwanderer wurden durch das neue Gesetz erleichtert, während der allgemeine Anwerbestopp weiterhin bestehen blieb (BAMF, 2005). Sprach- und Integrationskurse wurden verpflichtend vorgeschrieben (KURTOĞLU, 2008).

Trotz der beachtlichen Fortschritte auf integrationspolitischer Ebene blieben die sozialen Folgen der Migrationspolitik der letzten Jahrzehnte auch nach Beginn des 21. Jahrhunderts teilweise bestehen. Besonders türkische Migranten scheinen weiterhin eine der benachteiligsten Migrantengruppe zu sein. Sie arbeiten nach wie vor überwiegend im Niedriglohnsektor und türkische Frauen zeigen eine geringe Partizipation am Arbeitsmarkt (BAMF, 2010). Das Bildungsdefizit bleibt ebenso ungelöst. Hinsichtlich der weiterhin bestehenden Benachteiligungssituation ist es nicht verwunderlich, dass 60-70\% der Befragten angeben, sich als Ausländer diskriminiert zu fühlen (WILAMOWITZ-MOELLENDORFF, 2001, 2002). Hinsichtlich dem Zusammenhang von Sprache und Integration ${ }^{21}$ berichtet WILAMOWITZ-MOELLENDORFF: „Ein besonders wichtiger Indikator für den Integrationsgrad der Türken in Deutschland ist die sprachliche Integration. Im Arbeitsleben ist dabei in der Regel [...] Deutsch, in der Familie Türkisch die Umgangssprache. In der Freizeit besteht eher eine Möglichkeit der Wahl der Sprache [...]. Dass dort überwiegend vor allem von den Befragten türkischer Staatsbürgerschaft türkisch gesprochen wird, zeigt ein Integrationsdefizit. Solche Defizite deuten sich auch im Freundeskreis oder bei Freizeitaktivitäten an, wo Kontakte mit Deutschen seltener stattfinden als mit Türken." (WILAMOWITZMOELLENDORFF, 2002, S.1).

${ }^{21}$ Integration meint nach BERRY $(1974,1980)$ die Akkulturationsstrategie eines Individuums, das bestrebt ist, sowohl die eigene Kultur aufrecht zu erhalten, als auch den Kontakt zur dominanten Kultur des Ankunftslandes aufrecht zu erhalten, zu suchen und zu pflegen. Akkulturation meint nach (REDFIELD, LINTON \& HERSKOVITS, (1936) die Veränderung kultureller Muster von einer oder zwei aufeinander treffenden Kulturen, als Folge des Aufeinandertreffens (zum Beispiel durch Migrantion). 
Die Anschläge vom 11. September 2001, welche zu negativen Einstellungen der westlichen Gesellschaften gegenüber Muslimen führten (KURTOĞLU, 2008; VEDDER et al., 2007) und mediale Berichterstattung über sogenannte Brennpunktviertel mit hohem Ausländeranteil (z.B. die Rütli-Schule in Berlin-Neukölln; Der Spiegel, 31.03.2006), den Kopftuchstreit (KURTOĞLU, 2008), etc. rückte die (fehlende) Integration erneut in das öffentliche Interesse.

Doch die größtenteils negative Wahrnehmung in der deutschen Öffentlichkeit ist selektiv und erfasst die Situation nicht ganzheitlich. So gibt es auch positive Befunde zu berichten. WILAMOWITZ-MOELLENDORFF (2001) stellte in einer türkischstämmigen Population z.B. eine hohe Verbundenheit mit Deutschland (45-55\%), Gerechtigkeitsempfinden mit der Gesellschaftsordnung (90\%) und Zufriedenheit mit der hiesigen Demokratie (80\%) fest. Die Befragungswerte liegen teilweise über den Umfragewerten bei deutschen Teilnehmern. Ebenso beurteilen türkischstämmige Befragte ihre persönliche Lebenssituation, Zukunft und Vergangenheit überraschend positiv und zeigen hierbei kaum Unterschiede zur deutschen Gesamtbevölkerung (WILAMOWITZMoellendorfF, 2002). Die politische Partizipation von Mitbürgern türkischer Abstammung wächst: sie werden vermehrt Mitglieder in deutschen politischen Parteien, organisieren sich politisch selbst, lassen sich auf kommunaler Ebene in Ausländerbeiräte wählen. Dabei ist seit den 1980ern der Fokus politischer Aktivitäten immer weiter weg vom Geschehen im Heimatland, hin zur Situation der deutschen Gesellschaft und der Situation von Migranten in dieser gerückt (KURTOĞLU, 2008). Eine Mitgliedschaft in islamistischen Organisationen trifft hingegen nur auf einen verschwindend kleinen Teil der Migranten zu (KESIN, 1997; KURTOĞLU, 2008).

Der Integrationsdiskurs der letzten Jahre ist inhaltlich beeinflusst durch die Auseinandersetzung mit in Deutschland lebenden Muslimen. Besonders prägend gestaltete sich die Deutsche Islamkonferenz, die auf Initiative des damaligen Innenministers Schäuble (CDU) und die Weiterführung durch seinen Nachfolger de Maizière seit 2006 jährlich stattfindet. Sie soll dem Dialog zwischen Staat und Muslimen dienen und langfristig zu einem Gesellschaftsvertrag zwischen ihnen führen (siehe hierzu www.deutsche-islam-konferenz.de).

Ein besonderer Erfolg ließ sich Mitte dieses Jahrzehnts feiern: Islamunterricht in deutscher Sprache wurde in das Fächerangebot einiger Schulen 
und in die universitäre Ausbildung aufgenommen (KURTOĞLU, 2008).

\section{EXKURS: Was bedeutet Integration?}

Durch Wanderungsbewegungen kommt es zwangsweise zum Kontakt zwischen Menschen unterschiedlichen kulturellen Hintergrundes. Das Phänomen, dass bei diesen Kontakten Veränderungen der beteiligten kulturellen Muster zu beobachten sind, wird nach REDFIELD et al (1936) als Akkulturation bezeichnet und findet in Abhängigkeit zu den Einstellungen der Migranten und den Mitgliedern der Aufnahmegesellschaft in unterschiedlicher Weise statt.

Die Auswirkungen der Einstellungen der Migranten zur Akkulturation führen dazu, dass verschiedene Strategien in der Auseinandersetzung mit der interkulturellen Situation zum Einsatz kommen.

In diesem Zusammenhang hat BERRY $(1974,1980)$ den Versuch einer Klassifikation unternommen, wobei er betont, dass zwei Einstellungsdimensionen entscheidend sind:

Zum einen ist der Wunsch nach Erhalt der eigenen Kultur, zum anderen der Wunsch nach Interaktion mit der Aufnahmegesellschaft von Bedeutung.

Anhand dieser beiden Einstellungsdimensionen wird ein Feld aufgespannt, welches aus vier verschiedenen Umgangsstrategien mit dem Aufeinandertreffen der unterschiedlichen Kulturen besteht.

Bei der Auswahl einer dieser Strategien zeigen nationale und ethnische Identität einen gewissen Einfluss bzw. eine gewisse Bedeutung, insofern sie in Zusammenhang mit dem Wunsch nach Erhalt der eigenen Kultur oder eben der Veränderung dieser in Richtung der Kultur der aufnehmenden Gesellschaft wirken.

Anders betrachtet könnte man auch formulieren, dass ein Bestreben die eigene Identität zu erhalten oder eben zu verändern besteht, denn zu einem gewissen Grad fußt diese ja auf der Kultur.

In Abhängigkeit davon, wie weit die Bereitschaft ist, die eigene Identität einem Wandel zu unterziehen und wie das Bestreben, den Kontakt mit der Gastkultur zu pflegen, beschaffen ist, lassen sich, wie eben erwähnt, vier verschiedene Strategien fassen.

Ist das Bestreben des Individuums dergestalt ausgeprägt, dass der Erhalt der eigenen Kultur gepaart ist mit dem Wunsch nach Kontakt mit der 
einheimischen Bevölkerung, so ist gemäß BERRY die Strategie der Integration das Resultat.

Ist der Wunsch nach Kontakt hingegen nicht gegeben und die eigene Kultur soll erhalten bleiben, so wird die resultierende Strategie als Separation bezeichnet.

Sollte der Wunsch des Migranten sich hingegen als Distanzierung in beiden Dimensionen darstellen, also weder der Erhalt der Kultur, noch der Wille zur Kontaktaufnahme bestehen, wäre das Resultat ein kompletter Rückzug, auch als Marginalisation bezeichnet.

In der vierten und letzten Konstellation, der Assimilation, wird die eigene Kultur aufgegeben und der Kontakt zur ursprünglichen Gesellschaft zu Gunsten des Kontakts mit Mitgliedern der Aufnahmegesellschaft abgebrochen.

Ein schematischer Überblick der verschiedenen Akkulturationsstrategien ${ }^{22}$ nach BERRY findet sich in der folgenden Tabelle:

Tabelle 2 Akkulturationsstrategien nach $\operatorname{BERRY}(1974,1980)$.

\begin{tabular}{|c|c|c|c|}
\hline & \multicolumn{2}{|c|}{ Eigene Kultur erhalten } \\
\hline & & JA & NEIN \\
\hline Kontakt & $\mathrm{JA}$ & Integration & Assimilation \\
\hline Gastkultur & NEIN & Separation & Marginalisierung \\
\hline
\end{tabular}

Weiter ist interessant, dass ESSER in Hinblick auf die von ihm beschriebene Inklusion ein überaus ähnliches Schema beschreibt, obgleich in seiner Variante sehr viel deutlicher wird, dass Marginalität kaum attraktiv für die Migranten sein kann, weswegen bei ihm auch nicht der Begriff der Strategie fällt:

Als möglichen Bezugsrahmen für die Inklusion gibt ESSER vereinfacht die Aufnahmegesellschaft (bzw. ein Segment dieser) oder die ethnische Gruppe (als die Herkunftsgesellschaft, eine ethnische Kolonie oder als transnationales Netzwerk) an. Angesichts einer binären Unterscheidung nach Integration / keine

\footnotetext{
${ }^{22}$ Der Begriff Strategie wirkt hier umfassend, schließlich klingt es damit so, als würde es sich um ein absichtliches, gezieltes Verhalten, handeln. Das ist aber insbesondere bei der Marginalisierung, also einem Zustand des Ausschlusses, bzw. der „Kontaktlosigkeit“ wohl kaum zu vermuten. Der Eindruck, dass eine bewusste Entscheidung dazu führt, dass man marginalisiert ist, könnte entstehen, was wiederum implizieren könnte, dass man an diesem Zustand auch nichts verändern kann und er entsprechend etwa politisch belanglos ist.
} 
Integration ergeben sich damit diese möglichen Konstellationen einer SozialIntegration:

- Marginalität, als das Fehlen von (jeder) sozialen Integration. Als extremes Beispiel führt ESSER hierzu Marginalität in allen vier Bereichen der sozialen Integration an, wenn also „keine Sprache richtig beherrscht wird, nirgendwo eine akzeptable Position besetzt wird, keine sozialen Beziehungen unterhalten werden und man sich mit keiner Gesellschaft identifiziert." (ESSER, 2000: 287)

- multiple Inklusion, als soziale Integration in beide (bzw. allgemein mehrere) Bezugssysteme

- individuelle Segmentation, in der die soziale Integration auf die ethnische Gruppe bzw. Gemeinde beschränkt bleibt und

- individuelle Assimilation, in der nur die Aufnahmegesellschaft als Bezugsrahmen dient.

Gemäß den Untersuchungen von BERRY et al. (2006) erweisen sich diese verschiedenen Strategien als unterschiedlich erfolgversprechend bezüglich der Adaption, wobei eine erfolgreiche Adaption sich durch eine positive psychologische Adaption und eine positive soziokulturelle Anpassung auszeichnet.

Die Strategie der Integration ist dabei die hinsichtlich der Adaption wirksamste Alternative. Dabei sind die Dimensionen der Akkulturationsstrategien nicht in gleicher Weise förderlich für die Adaption:

Für die psychologische Adaption ist laut CEYLAN (2006) vor allem die Annäherung an die ethnische Gruppe verantwortlich (also der Erhalt der Kultur), wohingegen OPPEDAL et al (2004) zeigen konnten, dass die soziokulturelle Anpassung eher durch die Interaktion mit der nationalen Kultur bestimmt wird.

Es fällt auf, dass sich bei der Integration die Ausprägungen beider gleichermaßen positiv auf die Adaption auswirken. Es muss dabei beachtet werden, dass die Begriffe Assimilation und Integration im öffentlichen Sprachgebrauch häufig synonym verwandt werden, oder zumindest keine saubere Trennung erfolgt und der Erhalt der eigenen Kultur als Eigenschaft der Integration verloren geht. Verschiedene Untersuchungen weisen darauf hin, dass die Präferenz der ethnischen Kultur weder bedeuten muss, dass nur geringe oder keine Identifizierung mit dem Aufnahmeland vorhanden ist (VERKUYTEN UND YILDIZ, 2007), noch dass gar eine ablehnende Haltung gegenüber nationalen oder 
anderen fremdethnischen Gruppen zu Tage tritt.

Anders formuliert, ließe sich behaupten dass der Wunsch die ethnische Kultur zu erhalten weder mit Disidentifikation noch mit Separation gleich zu setzen ist.

Es muss jedoch davon ausgegangen werden, dass fehlende Integration dazu führen kann, dass nur eine geringe nationale Identität entsteht (Befunde hierzu bei PHINNEY et al. 2001) und es weiter zu negativen Einstellungen gegenüber den Mitgliedern der Aufnahmegesellschaft (BOHNER et al. 2008) sowie schließlich zu einer Orientierung, die nicht bikulturell geprägt ist, kommen kann (VEDDER et al. 2007).

Letztlich könnte man sagen, dass die oben aufgeführten Beobachtungen wiederum auf eine fehlende Integration hinweisen können.

In der öffentlichen Sicht, die häufig verzerrt wird durch übertriebene Berichterstattung über derartig auffälliges, aber eben an sich auch eher seltenes Verhalten ist entsprechend häufig die Meinung vertreten, dass die fehlende Integration Schuld der Migranten sei (YILDIZ, 2006).

In dieser Betrachtungsweise wird jedoch die Beteiligung der Aufnahmegesellschaft an der Integration vernachlässigt.

Mangelt es den Einwanderern jedoch an Unterstützung dahingehend, den Erhalt ihrer Kultur zusammen mit der Annäherung an die Aufnahmegesellschaft erlangen zu können, so werden die realisierbaren Strategien in ihrer Wahrnehmung auf die Auswahl zwischen Separation und Assimilation eingeschränkt.

ZICK (2010) hat festgehalten, dass die bevorzugte Strategie der Migranten (zumindest anfänglich) die der Integration ist.

Um die Bedeutung der Rolle der aufnehmenden Gesellschaft erfassen zu können wird an dieser Stellt das interaktive Akkulturationsmodell von BouRHIS et al. (1997) herangezogen, in welchem die Vorhersage von Adaptionsergebnissen nicht allein auf der Grundlage der Präferenzen der Migranten hinsichtlich einer der vier verfügbaren Strategien getroffen wird. Vielmehr wird bei BouRHIS et al. der Versuch unternommen den Adaptionserfolg aus der relativen Kompatibilität eben dieser Strategiepräferenz der Migranten und jener der Gastgesellschaft heraus abzuleiten.

Bedeutsam ist in diesem Zusammenhang, dass nicht nur die Vorliebe einer 
bestimmten Strategie, sondern auch die subjektive Wahrnehmung der bevorzugten Strategie der zweiten involvierten Gruppe wirksam ist.

Mit anderen Worten: Wenn es der Wille der Migranten, ist Integration zu erlangen, die Aufnahmegesellschaft jedoch das Ziel hat, dass Migranten sich assimilieren, so wird dieser Konflikt sich hinderlich auf die Adaption auswirken. Dies gilt auch, wenn der Wunsch der Aufnahmegesellschaft sich medial als Wunsch nach Assimilation darstellt und daher ähnlich wirksam sein, wie das tatsächliche, objektive vorhanden sein einer derartigen Präferenz.

Insgesamt sind also Einstellung und Verhalten der Migranten und der Aufnahmegesellschaft für eine erfolgreiche Adaption und Integration elementar.

\subsection{Migrationsdefinition(en)}

Migrationsdefintionen sind weitreichend inter- und intradisziplinär vorhanden. Relevante Definitionen verschiedener Disziplinen werden im Folgenden angeführt.

\section{Die individualistische Migrationstheorie von HARTMUT ESSER}

Die individualistische Migrationstheorie von Hartmut Esser, welche wohl als eine der bedeutenden Theorien im deutschsprachigen Raum in der Migrationssoziologie gelten kann, basiert auf der Tatsache, dass soziale Zusammenhänge über Aussagen auf der Ebene der Individuen erklärt werden können, einer Idee, die ESSER aus dem methodischen Individualismus, bzw. die diesen umfassenden kognitiven Theorie des Lernens entnommen hat.

Im Falle der Migration führt ESSER also alle sozialen Prozesse im Umfeld der Migration auf das Lernen und Handeln der individuellen Migranten zurück. Migration fasst ESSER (1980: 14ff) somit als einen Prozess der Eingliederung der Migranten in eine Aufnahmegesellschaft auf, wobei dieser Prozess die Gesamtheit der Teilprozesse der Herauslösung der Migranten aus ihren alten Rollenverbindungen (in ihrer Herkunftsgesellschaft) und auch der Knüpfung und Neuordnung neuer sozialer Beziehungen mit der Aufnahmegesellschaft umfasst. Esser bezeichnet diesen Prozess als Eingliederung.

Ebenso von Bedeutung sind in ESSERS Theorie die Begriffe der 
Akkulturation (als Prozess der Angleichung insbesondere im Hinblick auf kulturelle Eigenschaften der Migranten an die Aufnahmegesellschaft), der Integration (hier als regelmäßige Interaktionsbezüge zwischen Migranten und Einheimischen, wobei Integration erreicht ist, wenn ein Gleichgewichtszustand bei den Beziehungen zu den Einheimischen erreicht ist und keine Spannungen bestehen) und schließlich der Begriff der Assimilation (den man als einen mit der Akkulturation vergleichbaren Zustand im Hinblick auf Handlungen und interaktive Verbindungen mit dem Aufnahmesystem beschreiben kann).

Über diese drei Begriffe ist ESSER in der Lage, die Beziehung der Migranten zum gesellschaftlichen System der Aufnahmegesellschaft zu untersuchen, wobei er weiter ausführt, dass diese Prozesse sich zeitlich in der oben aufgeführten Reihenfolge ereignen.

Im Falle der Assimilation sind nach ESSER nochmals vier verschiede Dimensionen zu differenzieren, welche wiederum zwei verschiedenen Bezugsrahmen (zum einem einem relationalen Bezugsrahmen, zum anderen einem absoluten) zuzurechnen sind.

Die Dimensionen der Assimilation im relationalen Bezugsrahmen beschreibt ESSER als die soziale und die strukturelle Assimilation (u.a. die Einnahme einer sozialen Rolle oder einer gesellschaftlichen Position). Im absoluten Bezugsrahmen hingegen nennt ESSER die kognitive Assimilation (als die Übernahme/Akkumulation von Wissen) und die identifikative Assimilation (als die Übernahme von Werten und Bräuchen).

ESSER führt weiter aus, dass das Verhalten des Migranten in Abhängigkeit von Eigenschaften der Person und Eigenschaften der Umgebung unterschiedlich ausfällt.

In Bezug auf die Person, so ESSERS Hypothese, sind die (assimilative) Zielsetzung des Migranten, bzw. deren Anreiz (Motivation), die subjektive Hoffnung, durch bestimmte (assimilative) Handlungen dieses Ziel zu erreichen (Kognition), das Vertrauen in die eigene Handlung und die Kontrolle der Umgebung (Handlungsattribuierung), sowie die Kosten dieser Handlung entscheidend für das (assimilative) Verhalten (vgl. Personhypothese ESSER 1980: 211).

Im Hinblick auf die Umgebung lassen sich ebenso bestimmte Eigenschaften ausmachen, die sich (positiv oder negativ) auf das Verhalten des Migranten 
auswirken. Diese beschreibt ESSER in seiner Umgebungshypothese, welche besagt, dass der Migrant entsprechend des Verhältnisses von Opportunitäten (Möglichkeitenstrukturen, die sich begünstigend auf assimilative Handlungen auswirken, bzw. der Mangel an Alternativen zu assimilativen Handlungen) und Barrieren (solchen Begebenheiten, welche assimilativen Handlungen im Weg stehen) eher dazu neigt, assimilative Handlungen zu vollziehen, oder eben nicht (ebd.).

Aus den Handlungen des Migranten ergeben sich wiederum Konsequenzen für die Umgebung, so dass bestimmte Zusammenhänge zwischen Umgebung und Handlung festzustellen sind.

Auch hinsichtlich der Integration spricht sich ESSER dafür aus, in seiner Theorie eine Differenzierung vorzunehmen. Zunächst gilt es, zwischen Sozialintegration und Systemintegration zu unterscheiden, wobei die erstere die Beziehung von einzelnen Individuen zueinander über Einstellungen zum gesellschaftlichen System vermittelt, während die Systemintegration wiederum den Zusammenhalt des gesamten Systems (also sinngemäß eine gewisse gesellschaftliche Stabilität) betrifft. Wichtig ist dabei, dass diese Differenzierung nicht getroffen wird, weil diese beiden Formen der Integration voneinander unabhängig wären, sondern vielmehr eine Notwendigkeit darstellt, um Klarheit bei der Kommunikation bzw. der Untersuchung eines gesellschaftlichen Systems und der in ihm wirksamen Zusammenhänge schaffen zu können.

Die Sozialintegration verläuft nach Esser über die Mechanismen von Markt, Organisation und Medien.

Im Falle der Sozialintegration lassen sich erneut vier Dimensionen ausdifferenzieren, nämlich die Kulturation, Plazierung, Interaktion und die Identifikation.

Diese Dimensionen beschreiben

1. den Erwerb von Wissen (Humankapital, in einer der wichtigsten Formen etwa als Spracherwerb) als Teil der kognitiven Sozialisation,

2. die Plazierung in der gesellschaftlichen Ordnung (etwa die Einnahme eines Berufs und damit die Möglichkeit zur Akkumulation von ökonomischem Kapital sowie der Erwerb bestimmter Rechte und Pflichten, so etwa auch die Staatsbürgerschaft),

3. bestimmte Formen des sozialen Handelns wie sie etwa durch das Nutzen 
des erworbenen Wissens in Beziehungen zwischen den einzelnen Akteuren statt findet (bsp. in Form von Kommunikation) und

4. schließlich die Identifikation mit dem sozialen System (Gefühl der Heimat, aber auch Zustimmung zu bestimmten politischen Instanzen oder auch gesellschaftlichen Werten).

Da der Bezugspunkt der Sozialintegration sich in Form verschiedener Optionen für die Migranten darstellt, nämlich in der Form der Aufnahmegesellschaft, der Herkunftsgesellschaft und der ethnischen Gemeinde im Aufnahmeland (ESSER 2001: 19), können verschiedene Formen der Sozialintegration bestimmt werden:

Die Assimilation, welche die Integration in die Aufnahmegesellschaft bezeichnet und daher die Herkunftsgesellschaft vernachlässigt, die Segmentation, welche die Integration in die Herkunftsgesellschaft oder ethnische Gemeinde meint, die Marginalität, welche einen Zustand beschreibt, in dem weder eine Integration in die Aufnahme- noch in die Herkunftsgesellschaft bzw. ethnische Gemeinde statt findet, und schließlich der von ESSER als äußerst unwahrscheinliche und theoretische nur schwer bzw. nicht vollständig imaginierbare Fall der Mehrfachintegration.

Die Mehrfachintegration wird dabei von ESSER als äußerst unwahrscheinlich bewertet, weil sie zum einen sehr hohe Ansprüche mit sich bringt (Mehrsprachigkeit, doppelte Identität) und zum anderen mit sehr hohen „Aufwandskosten“ verbunden ist.

Da sowohl Marginalität als auch Segmentation aus normativer Sicht wohl kaum zu empfehlen sind (auf Grund ihrer potentiellen, destabilisierenden Wirkung für die Gesellschaft bzw. der desolaten Situation in welcher sich die gesellschaftlich ausgeschlossenen Wanderer befänden) liegt für ESSER somit die verbleibende Möglichkeit beinahe zwangsweise in der Assimilation, also der Angleichung der Migranten (in bestimmten, nicht notwendigerweise allen Eigenschaften) an die Aufnahmegesellschaft und ihrer Integration in die Aufnahmegesellschaft.

Die Assimilation kann ebenfalls in den Dimensionen kulturell, strukturell, sozial und emotional differenziert betrachtet werden (im Wesentlichen analog zu der bei der Integration getroffenen Unterscheidung). Dabei kann eine gewisse sequentielle Reihenfolge dieser Formen der Assimilation festgelegt werden: 
zunächst erfolgen die kulturelle und strukturelle Assimilation (also der Spracherwerb und die gesellschaftlichen Plazierung). Bei Erfolg können später die soziale und emotionale Assimlation stattfinden (also die Übernahme bestimmter Lebensformen der Aufnahmegesellschaft, wie z.B. das Verhalten bei Eheschließungen, und eine emotionalen Identifikation mit der Aufnahmegesellschaft.

Die besondere Bedeutung der strukturellen Assimilation (also dem Zugang zu (zentralen) Positionen in der Gesellschaft) hebt ESSER hervor.

In einem Punkt jedoch kann ESSER widersprochen werden. Betrachtet man beispielsweise die jungen Nachkommen der ehemaligen Gastarbeiter, mag die Begrifflichkeit von Assimilation im Sinne von ESSER hinsichtlich der Identitätsversicherung nicht so recht greifen. Hier wäre weniger von einer Assimilation denn von einer Neuentwicklung eines Gefühls, gar einer Neubildung von Identität die Rede. Dies begründet sich vor allem darin, dass eine Assimilation als ersten Schritt immer eine Destabilisierung fordert, um eine folgende Assimilation überhaupt erst möglich zu machen. Die Tendenz, die eigene Herkunft dabei zu idealisieren und damit interkulturelle Grenzen zu verfestigen, ist gegeben. Um dieses Selbst- und Fremdbild aufrecht, und damit stabil zu erhalten, ist jedoch viel Energie nötig, die, auch wenn sie un- oder unterbewusst erbracht werden kann, zu Stress führt. Die jungen, sogenannten „Deutsch-Türken“ verstehen sich jedoch meist nicht als assimilierte Deutsche oder als vollständige Türken, sondern bilden ein neues Identitätsgefühl heraus und damit keine Assimilation im Sinne ESSERS.

\begin{abstract}
Yet, as the essays by ØSteRgaARD-Nielsen and ElLis and KHAN in this volume demonstrate, the distinction that is commonly made between participation in a host state polity and practices directed towards transforming the home state is an artificial one. This is one of the many reasons why the concept of 'transnational communities', which has been coined largely by anthropologists and sociologists, should also be of interest to political scientists. Without the transnational perspective afforded by conceptual categories such as 'transnational community' or 'diaspora' it is difficult to theorize the ways in which political conditions and political activities in migration-receiving states are connected to dynamics of domestic political change and transformation in migration-sending states. (ADAMSON 2002: 155)
\end{abstract}

angesichts dessen plädiert ADAMSON dafür, das Konzept der "transational communities" in die Politikwissenschaften zu übernehmen, welches die (künstliche) Trennung zwischen der politischen Partizipation in Aufnahmeländern 
und Praktiken, die darauf abzielen, ein Herkunftsland zu transformieren, überwinden kann. Dies schafft wiederum die Möglichkeit Theorien aufzustellen, welche die politischen Zustände und Aktivitäten in Aufnahmeländern in Zusammenhang mit den Dynamiken des innenpolitischen Wandels und der Transformation in Sendeländern bringen.

One of the ways of modelling the relationship which exists between the home state, host state and transnational community is through a 'triadic model' which has been put forth by authors such as WEINER (1970) and SHEFFER (1986). The triadic model portrays the relationship between transnational communities, home states and host states as a threeway interaction that produces a variety of feedback and interaction effects. What this triadic model fails to capture, however, and what is clearly demonstrated by many of the case studies in this volume, is the degree of internal differentiation and complexity found in most transnational communities, and the resulting difficulty of making any concrete generalizations about their political activities (or lack of political activities, as most members of a community may well be largely apolitical). (ADAMSON 2002:158)

ADAMSON kritisiert am triadischen Modell des Verhältnisses zwischen transnationaler Community, Herkunfts- und Aufnahmeland, dass es nicht in der Lage ist, den Grad der inneren Differenzierung und Komplexität in transnationalen Communities zu erfassen, und somit auch die sich aus der Differenzierung und Komplexität ergebenden Schwierigkeiten die politischen Aktivitäten dieser Communities zu verallgemeinern.

The political activities of transnational communities take place within networks and structures that stretch across two or more states. As political actors situated at the intersections of states, members of transnational communities are able to reframe and rearticulate their political identities in ways that can either challenge or strategically reinforce hegemonic conceptions of national identity in both the home and the host states. The 'home', in a sense, becomes the transnational space' which is constructed and inhabited by the transnational community. Several of the case studies in this volume point to examples of strong political or religious identities within transnational communities which run counter to official constructions of national identity in those communities' home states. (ADAMSON 2002: 160)

Zudem hebt sie hervor, dass die Mitglieder von transnationalen Communities in einem gewissen Sinne im transnationalen Raum beheimatet sind, der durch die transnationale Community geformt wird, so dass die Mitglieder dieser Communities in der Lage sind, hegemoniale Konzeptionen von nationaler Identität strategisch zu stärken oder herauszufordern und zwar sowohl die des Aufnahme als auch die des Herkunftslandes. Dies ist zumindest in der Theorie möglich, wobei er jedoch nicht für jede transnationale Community gelten muss.

A second way that actors in migration-based transnational communities can organize to effect political transformation in their home state is by mobilizing to raise international awareness of the domestic political or economic conditions which they seek to change. The importance of ethnic lobbying has long been studied as a feature of US politics, but fewer studies have examined this process in other political contexts. (ADAMSON 2002: 162) 
ADAMSON merkt des Weiteren an, dass die Akteure in transnationalen Communities sich organisieren können, um die politische Transformation in ihren Herkunftsländern zu beeinflussen. Somit können sie etwa durch das Schaffen eines internationalen Bewusstseins für die innenpolitischen und ökonomischen Zustände, welche sie verändern möchten, in Aktion treten. ADAMSON schlägt deshalb vor, den Prozess des ethnischen Lobbyismus auch in anderen politischen Zusammenhängen (wie etwa einem transnationalen) zu untersuchen.

Transnational communities and the mobilization and transfer of material resources "A growing body of literature has begun to highlight the role that members of transnational communities play in providing material support for armed conflicts within the so-called homeland." (ADAMSON 2002: 165)

Das Wirken von transnationalen Communities kann laut ADAMSON auch in Form der materiellen Versorgung zur Unterstützung von bewaffneten Auseinandersetzungen im so genannten Heimatland in Erscheinung treten.

Some of the research on return migration demonstrates that those who do return often remigrate, leading MARGOLIS (1995), based on her research among Brazilian immigrants in New York City, to formulate the concept of "yo-yo migration" as yet another type. She contrasts this type of migration with cultural commuters or shuttle migrants "who regularly migrate back and forth between home and host country with no particular intention of staying in either place for good" (MARGOLIS 1995: 32). (BRETTEL 2000: 101)

BRETTEL et al. weisen darauf hin, dass verschiedene Autoren, darunter insbesondere MARGOLIS, bei der Untersuchung von Rückwanderern häufig beobachten, dass diese des öfteren erneut migrieren. MARGOLIS geht deswegen soweit, den Typus der "Yo-Yo-Migration" auszuweisen, eine Wanderungsart, die sich dadurch von anderen Formen der Mehrfachmigration abhebt, dass die Migranten regelmäßig zwischen Heimat- und Gastgeberland hin und her wandern, ohne dass eine bestimmte dauerhafte Aufenthaltsintention zu beobachten wäre.

Ruth MANDEL (1990) describes the pain and disorientation characteristic of adolescent Turkish returnees, and in another essay she alludes to the creation of a new ethnic category for Turks who have repatriated - Alamanyali, the "Germanlike" (MANDEL 1989). As such, rather than being accepted and respected, they are mocked. Similar categories exist for returned Portuguese migrants, be they the brasileiros of the nineteenth and early twentieth centuries, or the franceses of more contemporary times (BRETTEL 1979, 1986). (BRETTEL 2000: 101)

Weiterhin zeigt ${ }^{23}$ BRETTEL aber auch auf, dass die Rückkehrer in einigen

${ }^{23}$ Die Rückkehr in die Heimat schafft eine neue ethnische Kategorie mit Reaktionen wie Schmerzen und Desorientierung sowie das Gefühl des Nicht-Akzeptieret-Seins. Die Rede ist hier von einem invalidierenden Umfeld bei innerer Ambivalenz. Diese Begrifflichkeiten finden 
Fällen mit erheblichen Problemen konfrontiert werden, da sie in ihrem ehemaligen Heimatland nicht mehr als Angehörige des Staates anerkannt werden. BRETTEL verweist auf MANDEL, die im Falle adoleszenter türkischstämmiger Rückkehrer eine charakteristische Orientierungslosigkeit und schmerzhafte Erfahrungen schildert, welche den nunmehr als Alamanyali kategorisierten widerfährt. Nicht Akzeptanz und Respekt sondern Spott wird diesen Rückkehrern entgegengebracht. Analoge Beispiele lassen sich auch für portugiesischstämmige Migranten anführen.

Weiterhin sind mehrere Systematiken zur Einteilung verschiedener Migrationstypen bekannt ${ }^{24}$. Nachfolgend werden einige der meist genutzen Beispiele exemplarisch gebenannt:

\section{- Transmigranten (PRIES 2001)}

Eine nationale Migration ist weder einmalig noch unidirektional (vgl. PRIES 1997: 29). deshalb gelten die Migrantinnen und Migranten nicht mehr als in ihrem Heimatland Entwurzelte, die sich leidvoll Allmählich Im Aufnahmeland Assimilieren, Sondern auch als „Trans Migranten“, die über vielfältige Beziehungen sowohl im Aufnahmeland als auch in ihrem Herkunftsland verfügen (vgl. GLICK-SCHILLER/BASCH/SZANTON-BLANC 1997:121). Da sie sich sowohl mit Orten in ihrem Herkunftsland, als auch mit Orten in ihrem Aufnahmeland verbunden fühlen, konstruieren sie einen sozialen Raum, der sich zwischen diesen Orten Aufspannt (vgl. PRIES 1997:16). Wie „Trans Migranten“ begründen durch die Konstitution eines transnationalen Raumes eine transnationale Community (vgl. GoLDRING 180-182).

Davon ist auch ihre Identitätsbildung ${ }^{25}$ beeinflusst: Sie bilden multiple Identitäten aus, die sowohl in Herkunfts- als auch im Aufnahmeland Gründen (vgl. GoLDRING 1997:180-182). Paul Mecheril bezeichnet sie als „Trans kulturelle hybride“ (vgl. MECHERIL 1997: 294) damit muss sich auch der klassische Assimilation -/ Integrationsfokus erweitern.Trans Migranten wollen und können sich nicht assimilieren: „migrants have found full incorporation in the countries within wich they resettle either not possible or not desirable“" (GLICK-SCHILLER/ BASCH/ SZANTON-BLANC 1997: 126).

Eine völlige Assimilierung ist nicht wünschenswert, da die Migrantinnen und Migranten ihre Verbindung zum Herkunftsland aufrechterhalten wollen. Häufig ist es dringend notwendig, dass sie mit ihrem Herkunftsland in Verbindung bleiben, da sie im Aufnahmeland, eine äußerst unsichere Position haben.

Dabei nennen SCHILLER, BARSCH / SZANTON-BLANC zum einen die ökonomische und politische Unsicherheit, zum anderen auch den Rassismus und die allägliche Diskriminierung in den Aufnahmeländern. (GLICK-SCHILLER/ BASCH/ SZANTON-BLANC 1997: 123f.)

sich in der Symptombeschreibung z.B. von Persönlichkeitsstörungen in Folge von Stressverarbeitungsstörung wieder, worauf später im Verlauf dieser Arbeit näher eingegangen wird.

24 Je nach Disziplin und Schule variieren die Kategorien und Zuschreibungen. Hier werden nur exemplarisch einige Typen dargestellt. Eine umfassende Darstellung der aktuellen Literatur findet sich bei ZICK (2010: 126ff.)

25 Siehe Kulturelle Identitäten im Kapitel C - Fazit und Ausblick 


\section{- Globale Nomaden (LEIPRECHT und VOGEL 2007)}

Ein weiterer Typ [neben den Transmigranten, I.Ö. 2011], der 'globale Nomaden' genannt wird, ist prinzipiell offen für eine Vielzahl von Lebensorten. Die Netzwerke der 'Nomaden' sind eher internation und weniger lokal und national orientiert. (LEIPRECHT und VOGEL 2007: 27)

\section{-Lifestyle Migranten}

The ongoing quest for a better way of life explains the ambivalence that many of the migrants feel, while at the same time indicating that the initial destination may not be the final destination. While Korpela's Westerners admit to refining their image of the ideal place to live, other migrants are not so explicit about future migration. Instead, they stress that they are keeping their options for further migration open, emphasizing that if the place they had originally chosen becomes spoiled by extensive in-migration of other lifestyle migrants, they could move on to another place which offers the promise of the elusive good life. Lifestyle migration is a complex and nuanced phenomenon, varying from one migrant to another, from one location to the next. It holds at its core social transformation and wider processes; it is at once an individualized pursuit and structurally reliant; and it is a response to practical, moral and emotional imperatives. The chapters in this contribution shed some light on its diverse and complex nature, but even as we write, more locations are becoming involved, privileged actors are placing their own unique signature on migration and everyday lives within destinations are changing. (KAREN O'REILLY UND BENSON 2009: 1f.)

\section{-Raumpioniere (NOKIELSKI 2005)}

Bei der transnationalen Ruhestandsmigration handelt es sich im Unterschied zu den innerstaatlichen oder auch kleinräumigen Wanderungen älterer Menschen um einen relativ neuartigen, erst in der Spätphase der gesellschaftlichen Modernisierung an quantitativer und qualitativer Bedeutung gewinnenden Migrationstypus. [...] Er beschreibt anschließend das Ausmaß und die Gründe für die "Wohlstandsmigration" älterer Menschen, welche ihren Wohnsitz im Ruhestand dauerhaft oder auf Zeit in weit entfernte, klimatisch begünstigte Regionen verlegen. Diese Ruhestandsmigranten stellen aber keine Auswanderer im engeren Sinne dar, da sie auch weiterhin in ihre Herkunftsgesellschaft eingebunden bleiben und sich oftmals eine Rückkehroption offen halten. Der Autor bezeichnet sie daher als "Raumpioniere", die vielfach zwischen den Räumen ihrer alten und neuen Heimat pendeln, und somit auch den Begriff "transnational" begründen. Er diskutiert in diesem Zusammenhang die von L. Pries vorgetragene These, wonach sich mit den neuen Formen der Migration transnationale soziale Räume herausbilden, und bezieht sie auf das Phänomen der Ruhestandsmigration. (NOKIELSKI 2005:58)

Exkurs: Urbanes Zusammenleben

aus sozialwissenschaftlicher Sicht - WOLF- DIETRICH BUKOW

Unter Einbezug seiner These, dass allgemeine bzw. migrationsspezifische Mobilität einen prägenden Einfluss auf die Entwicklung der europäischen Stadt hatte, stellt Bukow eine Reihe von Punkten dar, die sich vor allem als Probleme in der öffentlichen Debatte präsentieren und sich des Weiteren durch eine recht beharrliche Reproduktion auszeichnen (vgl. Bukow, 2010: 38). Diese Punkte werden im Folgenden kurz beleuchtet. 
Familistische Fehlinterpretationen

Unter dem Problem der familistischen Fehlinterpretation versteht BUKOW den Umstand, dass das im 19. Jahrhundert propagierte Bild der Familie noch heute in der Politik Gang und Gäbe ist und somit auch heute im Übergang zur Postmoderne ein patriarchalisch und asymmetrisch geprägtes Modell weiterhin als adäquat und relevant dargestellt wird (vgl. BuKow, 2010: 40f). Ebenfalls Teil dieses Modells ist ein bestimmtes Set an Werten und Normen, welche in der idealisierten Familie des Familismus-Modells letztlich als Quelle des gesellschaftlichen Zusammenhalts fungiert.

BuKow weist an dieser Stelle darauf hin, dass dieses Modell von Familie wohl nie in der Realität angewendet wurde und des Weiteren, dass es angesichts der globalisierten Gesellschaft heute noch weniger zutreffen kann.

Während langsam Abstand davon genommen wird, dass die Familie Modell der Gesellschaft sein kann und zivilgesellschaftliche Konzepte in der Familie Anwendung finden, bleibt die Betrachtung von Mobilität davon unberührt.

Dass das veraltete Modell dennoch und entgegen objektiv beobachtbarer Sachverhalte Anwendung findet, führt Bukow einerseits darauf zurück, dass es stark in Traditionen verankert ist, etwa auch die der christlichen Kirche und andererseits darauf, dass „[...] es hier nicht um eine Modellierung von Wirklichkeit geht, nicht um deren Gestaltung, sondern um deren Definition." (BuKOW, 2010, S. 42)

Methodologischer Nationalismus

Bukow spricht hier die kaum überwindbare Gleichsetzung von Staaten bzw. deren Gebiet und einer Nationalgesellschaft an, einer Verknüpfung, welche gerade im Kontext von Mobilität bzw. migrationsspezifischer Mobilität zu einigen grotesken Wendungen in der öffentlichen Debatte führt.

Insbesondere zeigt BuKOW auf, dass die Askription von Fremdheit auf Menschen mit Migrationsgeschichte auch über mehrere Generationen hinweg wirksam bleibt. Ähnliches gilt für die Abwertung von Bildung bei Menschen mit migrativem Hintergrund, insofern dieser von der Bildung bzw. von bestimmten religiösen Mustern im Vergleich zur postulierten heterogenen Nationalgesellschaft 
abweicht (vgl. BuKow, 2010, S. 42ff). Das Fortbestehen von methodologischem Nationalismus sieht BuKow auch im wissenschaftlichen Diskurs. Da sich dies auch auf das Verständnis von interkulturellen Kompetenzen (im Wesentlichen wie Fremdsprachenkenntnisse ein Mittel zur besseren Kommunikation) auswirkt, kommt es dazu, dass Menschen erst einmal zu „echten Fremden“ abgestempelt werden, bevor sie nach der Überquerung der Staatsgrenze die Chance erhalten, "echte Deutsche" zu werden (vgl. Bukow, 2010, S.44).

Die Folge des methodologischen Nationalismus liegt für Bukow darin, dass mit dem Postulat der internen Homogenität der Nationalgesellschaft als auch dem Postulat der Homogenität „der Fremden“ ein Erkennen von globalgesellschaftlichen Verflechtungen verhindert wird. Dies gilt auch für die Sichtweise, dass die Multikulturelle Gesellschaft gescheitert wäre. Eine Auseinandersetzung mit einer diversifizierten Gesellschaft bleibt laut Bukow in der öffentlichen Debatte aus (vgl. ebd.: 45).

Kulturalistischer Fehlschluss

„Die Bedeutung expliziter kultureller und religiöser Deutungsmuster werden in ihrer Funktion für die Steuerung einer Gesellschaft immer wieder überschätzt." (Bukow 2010: 45)

Mit dem kulturalistischen Fehlschluss beschreibt BuKow den Umstand, dass Kultur und Religion bzw. kulturelle und religiöse Deutungsmuster in ihrer Funktion zur Steuerung und in ihrer Bedeutung für den Aufbau der Gesellschaft stetig überbewertet wurden und trotz des Übergangs in die Postmoderne, der mit einer Rückbildung von Nationalstaaten und einem Rückzug dieser aus sozial- und kulturnahen Bereichen einhergeht, auch weiterhin überbewertet werden.

Selbst das zu beobachtenden Phänomen, dass mehr und mehr religiöse und kulturelle Institutionen unter einem Rückgang an Interesse und Mitgliedern leiden, was wiederum dazu führt, dass die Institutionen ihre Programme „verweltlichen“, kann an diesem Phänomen nichts ändern.

Dabei wird jedoch zu Gunsten einer postulierten Staats-Gesellschaftskonstituierenden Eigenschaft von Kultur und Religion deren Bedeutung für die Konstitution der Subjekte einer Gesellschaft gemindert.

Der Grund dafür, dass Religion und Kultur in einer derartigen Weise in der 
öffentlichen Debatte in Erscheinung treten, liegt nach Bukow darin, dass Kulturen und Religionen als geschlossene Systeme behandelt werden, die eng mit Prozessen der gesellschaftlichen Entwicklung verknüpft sind, wobei der Kulturbegriff in einen Zivilisationsbegriff überleitet. Damit können Religion und Kultur letztlich zur Unterscheidung von eigener, fortschrittlicher Kultur und fremder, vorsäkulärer Kultur instrumentalisiert werden (BuKOW, 2010: 48f).

Da durch das somit entstehende Muster sowohl die Überlegenheit der eigenen Kultur und damit die Erwartung nach Angleichung an diese durch den Allochtonen bestärkt werden kann, als auch etwaige negative Askriptionen gegenüber den Fremden gerechtfertigt werden können, zieht BuKow das Fazit, dass „[...] der Fehlschluss keine irgendwie geartete Fehlleistung darstellt, sondern tatsächlich eine ethnozentrische Logik besitzt [...]" (BuKow, 2010: 50).

Der kulturalistische Fehlschluss kann entsprechend dazu beitragen, dass bestehende Machtstrukturen gefestigt werden und ist damit nicht nur Bestandteil der nationalen Erzählungen, sondern auch bestimmendes Element des modernen Kulturrassismus.

Der Sesshaftigkeitsmythos

Bukow ist der Ansicht, dass Sesshaftigkeit in der heutigen Zeit nicht adäquat verstanden wird, zum einen, da sie häufig als Normalzustand angesehen wird und zum anderen eher als emotional denn als rational wahrgenommen wird. Bukow hält fest, dass Sesshaftigkeit ein Konzept ist, das vor allem mit der zunehmenden Bedeutung von immobilen Produktionsmitteln an Bedeutung gewonnen hat, einem Umstand, der in modernen Gesellschaften zusehends weniger zutreffend für einen Großteil der Bevölkerung ist. Dazu kommt, dass Bukows Meinung nach die Begriffe Sesshaftigkeit und Nomadentum als komplementäre Begriffe eines bipolaren Schemas verwendet werden, obgleich man wohl eher von einem kontinuierlichen Übergang zwischen diesen beiden Zuständen auszugehen hat (vgl. Bukow 2010: 50f).

BUKOW stellt daher folgende exemplarische Formen der Mobilität als Teil des oben erwähnten Spektrums dar (vgl. ebd.: 51f): 
- generationenübergreifende Standorttreue (großindustrielle Familien)

- subjektiv empfundene Standorttreue (mehrfaches „endgültiges“

Umziehen, touristische Mobilität)

- sequenzielle Mobilität (saisonales Pendeln)

- der „klassische Migrant“ (Gastarbeiter, der im Aufnahmeland sesshaft geblieben ist)

- serielle Mobilität (sinngemäß jeder Lebensabschnitt in einem anderen Land)

- generationenübergreifende Mobilität (mit den Eltern aus $A$ nach $B$, zum Studium nach C, zwecks Arbeit nach D usf.)

Als außerhalb dieser Spannbreite zu verorten betrachtet BuKOW den tatsächlichen Nomadismus in klassischer (Naturbedingungen folgend) und modernerer Form (Verfolgung und Kriegen entfliehend), sowie die zirkuläre Mobilität (illegale Migration, um „zu Hause“- gebliebene Familien zu versorgen).

Gemäß der Argumentation, dass Mobilität in einer gewissen Form Teil der Lebenswelt eines Großteils der Weltbevölkerung ist (besonders bedeutsam nach Bukow sind dabei die Formen der sequenziellen und der seriellen Mobilität), ergibt sich, dass eigentlich auch Abstand von den Überlegungen genommen werden müsste, dass Sesshaftigkeit (fast zwangsweise) zu einer stabilen Lebenslage und der Herausbildung bürgerlicher Tugenden führt sowie am anderen Ende des Spektrums, dass eine mobile Lebensweise zu Verirrungen und Orientierungslosigkeit führt.

Stattdessen sollte man nach Bukow davon ausgehen, dass auch mobile Menschen durchaus in der Lage sind, eine stabile Identität und kulturelle Zugehörigkeit zu generieren.

Wird der Sesshaftigkeitsmythos überwunden, so wird der Blick frei gegeben auf ein Kontinuum, das vom Nomadentum bis zur Sesshaftigkeit reicht und das verschiedenste Formen von Mobilität und mobiler Existenz umfasst, die jeweils assoziiert sind mit einem Satz an Handlungsanweisungen für die unterschiedlichsten Situationen.

Den Umstand, dass bestimmte Formen der Mobilität zu bestimmten Zeiten normativ aufgewertet wurden führt Bukow darauf zurück, dass diese Formen unter gewissen gesellschaftlichen Bedingungen vorteilhaft waren. 
Überwindet man das Kulturprogramm, kann man erkennen, dass Mobilität und Migration eigentlich die Norm darstellen und nicht, wie dies aus deutscher Sicht die längste Zeit betrachtet wurde, eine besondere ausnahmesituation.

Im gesellschaftlichen Umgang mit Mobilität, vor allem hinsichtlich gesellschaftlicher Alltagspraxis, kommt es zu paradox erscheinenden Widersprüchen: einerseits ist Mobilität ein bekanntes Phänomen, mit dessen Umgang genügend Erfahrungen vorliegen, andererseits ist Mobilität gerade wenn sie von weniger Privilegierten ausgeht ein Problem, dem Einhalt geboten werden muss. Die Folge davon ist dass manche Formen der Mobilität ignoriert und ausgeblendet, andere hingegen eher beachtet werden. Der Grund dabei ist, dass traditionelle Vorstellungen von gesellschaftlichem Zusammenleben und Ordnung explizit durch Mobilität bedroht wirken; der sich eigentlich vollziehende Wandel hin zur Postmoderne bleibt dabei verkannt.

Bukow führt aus, dass spätestens in der Postmoderne nicht mehr die Familie, sondern der Diskurs zur Orientierung dient und Informations-, Orientierungs-, Sozialisations- und Deutungskapazitäten bietet.

Standorttreue als Maß ist nicht länger angebracht, Mobilität einer gewissen Intensität muss zum Maßstab werden.

Diversität und Mobilität werden von verschiedenen gesellschaftlichen Institutionen unterschiedlich schnell und effektiv angenommen: bestimmte Infrastrukturen sind im Geiste gesellschaftlicher Homogenität errichtet worden und teils nur schwer umzustellen (Quartiere und Bürokratie etwa sind besonders unveränderlich).

Dort, wo solche formalen Systeme lebende, agierende Systeme sind vollzieht sich der Wandel deutlich schneller und leichter. An diesen Orten kann Diversität als Ressource erkannt und genutzt werden.

Im Gegensatz dazu scheint sich der öffentliche Sektor kaum verändern zu lassen, da er ohne effektive, situationsadäquate Steuerung Bestand hat. Ziel der Verständigung ist es dann, formale Bereiche auf interne und externe Heterogenität auszurichten, was gleichbedeutend damit ist, das Andere extern und den Anderen intern anzuerkennen und somit Unterschiedlichkeit fair zu behandeln. Die nationalen Erzählungen und öffentlichen Debatten stehen dem allerdings entgegen. 
Zunehmende Mobilität und Fortschritte in der Kommunikation führen dazu, dass die Individualisierung einen deutlichen Schub erfahren hat. Dieser Individualisierung muss durch Neuerörterung von gesellschaftlichen Maßstäben Rechnung getragen werden.

Frühere Maßstäbe der Orientierung schrumpfen zu statistischen Werten (Schicht, Klasse, Berufsgruppe), so dass Mobilität und Migration eigentlich die Norm darstellen und nicht, wie dies aus deutscher Sicht die längste Zeit betrachtet wurde, einen (problematischen) Sonderfall. Der langsam voranschreitende Perspektivwechsel wird durch die beobachtbaren Globalisierungsprozesse beschleunigt. Insgesamt wird ersichtlich, dass Mobilität zunimmt: sie findet auf höherem Niveau, an mehr Orten und über mehr soziale Gesellschaftsebenen hinweg statt.

Bukow postuliert, dass politische Diskurse dem öffentlichen Alltagsleben hinterher hängen, insofern als im Altag der Umgang mit Diversität bereits natürlich geworden ist. Das Versagen der nationalen Erzählung im Kontext der Diversität der Gesellschaft sowie die Unfähigkeit der öffentlichen Debatten und insbesondere der politischen Debatten den Schritt in die Postmoderne zu vollziehen, hat häufige Ausuferungen in rechtspolitische Gefilde zur Folge, da die politischen Experten beim Versuch, den Übergang in die Postmoderne zu vollziehen an ihre Grenzen stoßen und schließlich diskursiv ausweichen.

Erfolgt dieses Ausweichen in die politische Rechte, so wird durch diese politischen Experten unreflektiertes rechtes Gedankengut in die gesellschaftliche Selbstinterpretation hineingetragen.

Daraus ergeben sich jedoch deutlich kontraproduktive Konsequenzen, wenn etwa die den Allochtonen auferlegten Bilder zu Selbstbildern (Bukow, 2010: 97) werden und es damit tatsächlich zu Zerwürfnissen in der Gesellschaft kommt.

Bukow nennt als Gründe für die wenig vorhandene reflexive Vernunft der Politik:

Freilich muss man in Rechnung stellen, dass diese „retrospektive“ bzw. nostalgische Ausrichtung [der Politik] keineswegs bloß aus Überforderung geschehen ist, sondern mitunter auch absichtsvoll und gezielt gemeint ist, dient sie doch auch dazu, die Zuwanderung bevölkerungspolitisch zu kontrollieren und in Richtung Unterschichtung weiterhin dienstbar zu machen. (BuKow 2010: 96) 
BuKOW ruft daher zu politischer Vernunft auf in der Hoffnung, dass diese es ermöglichen kann, den Übergang in die Transmoderne zu vollziehen.

Eine „Ausländer“-fixierte Integrationsdebatte [...] geht [...] zwangsläufig in die falsche Richtung, weil sie erstens ein wirklich Fremdes im Gegensatz zu einem wirklich Eigenen unterstellt und weil sie damit zweitens das Eigene über das Fremde erhebt. Es kommt statt dessen darauf an, die sich ausbreitende (hybride) Diversität und die damit verbundenen neuen (virtuellen) Repräsentationsformen von gesellschaftlicher Wirklichkeit zu realisieren und in ihrer Relevanz für das Zusammenleben neu zu durchdenken.(ebd.: 97)

Eine Anerkennung von Diversität würde in einer solchen Debatte nicht stattfinden können, denn sie wäre komplett von der ihr innewohnenden nationalistischen Fundierung überdeckt.

Als wünschenswert hingegen stellt sich nach Bukow im Gegensatz dazu eine Zivilgesellschaft nach dem Vorbild der Stadtgesellschaften dar,

[...] in der die Spielregeln formaler Zusammenarbeit nicht von wem auch immer, erlassen, werden, sondern unter breiter Beteiligung ausgehandelt werden. Gefragt sind erweiterte individuelle Spielräume zur Ausarbeitung persönlicher Lebensstile und Beziehungen sowie einer neu sensibilisierten und respektorientierten politischen Kultur. (ebd.: 97f)

Vorbildlich könnten dafür die Stadtgesellschaften insofern sein, als sie formale Systeme zur Ordnung des Zusammenlebens herausgebildet haben, die ohne die Notwendigkeit von persönlicher Bekanntheit oder von Verstehen auskommen.

Auch auf individueller Ebene muss aber nicht nur die eigene Individualisierung, sondern auch die des Anderen zu akzeptieren und zu respektieren sein, denn erst dann würde sich formale Gleichheit einstellen. Diese formale Gleichheit wird aber durchaus noch von vielen gefürchtet: der Schritt in die Transmoderne steht noch bevor.

Das Ziel, Integration nicht länger als Annehmen westlicher Werte, sondern als das Schaffen „einer globalen Zivilgesellschaft und [...] globale[r] Gerechtigkeit als Fairness“(Bukow 2010: 100 Herv. i. Ö.) zu verstehen und zu verbreiten soll damit nicht nur Aufgabe moderner Politik, sondern auch Forschungsinteresse der Wissenschaft sein, so BuKow's Aufruf.

Entscheidend für die Zählebigkeit der nationalen Erzählungen ist nach Bukow die Entwicklung der Moderne bzw. das Projekt der Moderne (ebd.: 209). 
Die Entstehung der nationalen Erzählungen ging zeitlich mit der Aufklärung einher, nach Bukow nicht zufällig. Besonders ausgeprägt ist dieser Nationalismus in jungen Staaten (eben Deutschland, aber auch nach Deutschland, den GUSNationen und der Türkei, aus denen die Haupteinwanderer stammen).

Ziel der Aufklärung war die (endgültige) rationale Durchdringung der Welt. Notwendig für dieses Projekt war eine entsprechende Macht zur Durchsetzung (in/über Politik, Wissenschaft, Bildung). Besonders folgenreich für das Projekt der Moderne ist die nationale Erzählung zum Zweck der Konzentration und Verdichtung (in diesem Sinne Reduktion) der Anderen. Denn mit der Kategorisierung in nationale Inklusion/Exklusion erfolgt stets eine Positionierung/Wertung. Diese Eigenschaft der nationalen Erzählung behält laut BukOW bis heute eine gewisse Attraktivität bei, denn: „Sie [die nationalen Erzählungen] bieten sich für eine starke Selbstinszenierung geradezu an."(ebd.: 211)

Die ursprünglich eher positive Wirkung der nationalen Erzählungen, die Leere zu füllen, welche in der Emanzipation von feudaler staatlicher bzw. christlich-kirchlicher Bevormundung entstanden war, führte durch die Überheblichkeit der Moderne

[...] faktisch nur zur Polarisierung zwischen überwölbenden kulturellen Erzählungen und den sie tradierenden Experten einerseits und dem Rest der Welt. Aber in kritischen Augenblicken wurde daraus alsbald ein ubiquitärer Deutungsschirm, der insbesondere in den Kriegen die gesamte einheimische Bevölkerung überspannte. (ebd.: 212)

Aus Bukows Sicht stellt die nationale Erzählung ein Reservoir an sozialen Mythen und nationalen Sprüchen dar, das in verschiedenen Krisen genutzt werden kann, um zu intervenieren.

Es sind die zitierenden Wiederholungen, welche die nationalen Erzählungen immer wieder verfestigen, aufs Neue möglich werden lassen, da die öffentlichen Debatten nicht am Maß der fortgeschrittenen Alltagspraxis gemessen werden (Vgl. Bukow 2010: 215). 
In der Öffentlichkeit werden immer wieder rassistische Kontextualisierungen vorgenommen, obwohl die Bedingungen für ein erfolgreiches Miteinander eigentlich günstiger geworden sind. Millieurassistische Interventionen gefährden das Miteinander, obwohl ihnen massiv widersprochen wird. Nationale Erzählungen erscheinen unendlich zählebig, obwohl sie erkennbar unzeitgemäß sind, ja schon von Beginn an nicht gehalten haben, was sie versprachen. Die in der Öffentlichkeit gepflegten nationalen Erzählungen wirken also nicht nur verdrängend, sondern verführen auch zu Selbstgerechtigkeit (wie dies die amerikanische Zivilreligion belegt), mach[en] erfahrungsblind (wie das die Ignoranz gegenüber indigenen Völkern zeigt), polemisch (wie das der von Samuel Huntington angezettelte Kampf der Kulturen illustriert \{Huntington 2005\}) und aggressiv (was mit der weltweit zunehmenden Zahl von Konflikten, Kriegen und Flüchtlingen zu belegen ist). (Bukow 2010: 215)

Experten können in öffentlich geführten Debatten angesichts der Unsichtbarkeit der nationalen Erzählung mit Ideen, wie der Entstehung von Parallelgesellschaften, erheblichen Schaden anrichten. BuKOW kritisiert weiter, dass die Inszenierung solcher Debatten häufig zur Verschleierung von Alltagspraxen führt (Vgl. BuKow 2010:218f).

Diese Verschleierung ermöglicht es dann auch, dass die erheblichen Widersprüche zwischen der gelebten Praxis auf der einen und dem Migrationsdiskurs, der diese beschreibt, auf der anderen Seite bestehen bleiben können.

Politische und öffentliche Praxis halten nicht mit der Entwicklung der Praxis des Alltags Schritt. Die Normalität, die der Wandel und das mehr oder minder distanzierte Neben- und Miteinander der verschiedenen Mitglieder der Gesellschaft darstellen, also die Alltagspraxis, wird nicht wirklich im öffentlichen Diskurs, wie ihn gesellschaftliche Akteure und Experten führen, repräsentiert, wo nach wie vor die jüngste Gruppe an Zuwanderern zum Problem stilisiert wird, indem man sie zur heterogenen Masse degradiert.

BukOW räumt ein, dass dies auch zusehends einfacher geschehen kann, da größere religiöse und sprachliche Distanz zwischen Zuwanderern und Autochtonen die kollektive Gleichmachung dieser Zuwanderer erleichtert.

Somit schließt BuKOW:

Es ist im politischen Diskurs ausgemacht, dass in Deutschland ein massives Integrationsproblem besteht und dass dieses Problem durch die fremde Ethnizität und letztlich durch die fremde Religion, nämlich den Islam, provoziert wird. (ebd. 2010: 219)

Somit wird der Islam zum Sinnbild der Fremde. (vgl. Bukow 2010: 219) 


\section{Alter}

\subsection{Definition}

Die Weltgesundheitsorganisation (WHO) definitiert als Alt, wer das 65. Lebensjahr vollendet hat. Im medizinischen und psycho-sozialen Kontext wird in Deutschland und Amerika von einem „geriatrischen Patienten“ erst ab dem Alter von 70 Jahren gesprochen. Auch kann unterschieden werden zwischen den Begrifflichkeiten 'biologisches Alter' und 'biografisches Alter'. Da das Altern ein sehr individueller Vorgang ist, lässt sich keine Festlegung finden, die eine klare Altersgrenze für 'das Alter' angibt. In der vorliegenden Arbeit werden Menschen mit Zuwanderungsgeschichte ab dem 55. Lebensjahr als 'Alte Migranten' betrachtet, da sie zumeist bedingt durch ihre Biographie sehr jung das Arbeitsleben begonnen haben, gleichzeitig wenig oder kaum Wert auf Gesundheitsfürsorge gelegt haben und dadurch in den meisten Fällen 'vorgealtert' sind. Dies wird im weiteren Verlauf dieses Kapitels ausführlich beschrieben und belegt mit dem Ziel einen Begriff für den 'alten Migranten' herauszuarbeiten.

\subsection{Situation alter Migranten in Deutschland}

Obwohl in einem der vorherigen Kapitel angeführt wurde, dass es zum Selbstverständnis der türkischstämmigen Menschen in Deutschland gehört, sich um die 'Alten' zu kümmern, verändern sich deren lebensweltliche Netzwerke stark in der Art, dass sie im Alter immer weniger stützend funktionieren. Bereits durch die Migration selbst haben sich manche Strukturen, Rollen- und Aufgabenverteilungen in den traditionell orientierten Familien verändert:

$\mathrm{Zu}$ den klassischen Kennzeichen von Familie gehören räumliche Nähe und direkte Gemeinschaft, also 'face-to-face'-Beziehungen im wörtlichen Sinn. Aber unter Bedingungen der transnationalen Migration, der institutionell gelenkten und beschränkten Wanderungsströme treffen wir auf weite Entfernungen und häufige Trennungen - Männer von ihren Frauen, Eltern von ihren Kindern, Geschwister von den Geschwistern getrennt -, oft über viele Jahre sich hinziehend, nicht selten auch wechselnde Konfigurationen annehmend. Damit werden die Gestalt und Bedeutung dessen, was weiterhin 'Familie' genannt wird, unter der Hand neu definiert und interpretiert. Galt früher als Liebesbeweis, daß man zusammenbleibt, was immer auch kommt, so finden wir in der globalisierten Welt zunehmend das Gegenteil als Gebot: Wer seine Familie liebt, der verläßt sie oder teilt sie auf in wechselnden Formen, um anderswo die Grundlage für eine bessere Zukunft zu schaffen. (BECK-GERNHEIM 2004: 46) 
In numerous social contexts migrants from Turkey were treated by the general public as a problem, a burden or as being deficient. In the 1980s a prominent German politician addressed the issue of migrants in Germany as a "Turkish problem" (TEPECIK 2002: 36).

[...]

The majority of migrant workers of Turkish descent came to Germany for economic reasons. Most of them stemmed from the lower levels of the social strata and from the rural areas of Turkey. Because the receiving country was only interested in physical laborers at the time and not in academic or occupational qualifications, many of those recruited to Germany stemmed from Turkey's lower socio-economic strata. In spite of these families' low socio-economic resources and this generation's frequently quoted disinterest in education, a number of their children have successfully obtained a German education and achieved upper level educational degrees.

(TEPECIK 2009: 372-374)

Hinzu kommt das Gefühl bei den alten Migranten, für lange Zeit als ein 'Problem' verstanden worden zu sein. Dieses Gefühl verstärkt den Eindruck 'nicht gewollt' zu sein und kann zu ungemeiner emotionaler Belastung führen.

\section{Konzepte älterer Migranten}

„Altwerden in der Fremde ist eine Metapher für die Beschreibung der Lebenssituation altgewordenen [sic] Migranten, die in der Regel schon seit dreißig und mehr Jahren in der Bundesrepublik leben." Für diese Menschen ist also das Migrationsland fremd geblieben (ERTL 2001: 124).

Meine Frau sagt mir

Kehren wir zurück

Du hast Sehnsucht

nach deiner Kindheit

Ich denke an meinen Sohn

und möchte nicht

daß er Sehnsucht hat

nach seiner Kindheit

Und sage: Diesen Winter

bleiben wir noch

KUNDEYT ŞURDUM

(zit. in: MÜLLER-WILLE 2001: 18) 
Die meisten türkischen Migranten wollten ursprünglich nicht dauerhaft in Deutschland bleiben, sondern planten ihren hiesigen Aufenthalt lediglich vorübergehend zur finanziellen Besserstellung als Arbeitsemigranten bzw. Gastarbeiter ein. Dementsprechend gering war das Bedürfnis, sich dem Gastland anzupassen und darin zu integrieren (KOPTAGEL-ILAL 1999: 323). Mit dem Anwerbestopp von $1973^{26}$, nach welchem eine neuerliche Einreise in die BRD nach zwischenzeitlicher Rückkehr in das Herkunftsland nicht mehr möglich war, änderten jedoch etliche Migranten die ursprünglichen Heimkehrpläne. Sie holten nun ihre Familien aus den Heimatländern nach und steckten sich als ein neues Lebensziel, den Kindern in Deutschland eine möglichst gute Ausbildung zu ermöglichen, die ihnen später in den Herkunftsländern eine gesicherte Existenz sichern sollte (MüLLER-WILLE 2001: 23). Dennoch ist bis ins Alter der Wunsch nach einer Rückkehr in die Heimat durch erinnerungsbedingte emotionale Bindungen vielfach lebendig geblieben. Etliche türkische Migranten haben sich dort Häuser als Altersruhesitz errichtet. Zudem wünschen auch viele ältere Türken, in der Heimat beerdigt zu werden (MüLLER-WILLE 2001: 23).

Auffällig ist vielfach die Diskrepanz zwischen den Vorstellungen älterer Migranten vom Heimatland mit dem heutigen Ist-Zustand in der Türkei: Türken im Alter von 50 bis 60 Jahren fühlen sich in der Regel noch sehr stark traditionellen Werten verhaftet, die sie in der, einem rapiden Wertewandel unterliegenden alten Heimat so nicht mehr vorfinden - wie in Deutschland stehen auch dort nicht mehr der unbedingte Zusammenhalt der Familie und die Orientierung am Gemeinwohl an oberster Stelle der Wertorientierung, sondern zunehmend der Individualismus und die Selbstverwirklichung des Einzelnen (s. z.B. AWO 1996: 5; AL-BARGHOUTI 2001: 44f). Ähnliches erleben ältere türkische Migranten mit dem einstigen Freundeskreis. Im Gegensatz zu der in der Ferne glorifizierten, warmen Atmosphäre müssen sie bei Besuchen vielfach feststellen, dass die Kontakte zu Verwandten und Freunden aufgrund der seltenen Kontakte zwischenzeitlich merklich abgekühlt sind und sie überdies den sozialpolitischen Wandel in der Heimat samt damit zusammenhängenden Änderungen des Lebensstils in den vergangenen Jahren nicht miterlebt haben (KOPTAGEL-ILAL 1999: 326). Landflucht und Verstädterung haben in der Türkei mittlerweile zu starken Veränderungen in

26 MÜLLER-WiLLE nennt hier im Widerspruch zu anderen Quellen das Jahr 1974 (2001: 23). 
den Herkunftsgemeinden geführt, die in ihrer ursprünglichen Form heute nicht mehr existieren. Damit sind die Migranten in ihren Heimatgemeinden mittlerweile zu Fremden geworden, wie der für sie dort häufig verwendete Ausdruck "Almanyalı" (= Deutschländer) unterstreicht - sie erleben also eine doppelte Entwurzelung (HAMBURger BeHÖRDE FÜr ARBEIT, GesundheIT UND SOZIALES 1998: 17f).

Allerdings gibt es auch Hinweise, dass sich diese einstigen Konzepte älterer Migranten allmählich wandeln: Laut BÜKRÜCÜ (2001) wollen inzwischen ca. 90\% ihren Lebensabend in Deutschland verbringen. Gründe sind ihre inzwischen hier verwurzelten Kinder sowie der gesundheitliche Aspekt, da die medizinische Versorgung der in der Türkei weit voraus ist (BÜKRÜCÜ 2001: 26). Diese Einschätzung bestätigte auch die Befragung der an der Erprobung des im folgenden der Arbeit noch näher erläuterten DRK-Medienkoffers ('Infokoffer') durch die Kreisverbände Reutlingen, Stuttgart und Tübingen zwischen Juni 2000 und Mai 2001 (BRAUN ET AL 2001: 80).

Im Vergleich zu der bundesdeutschen Bevölkerung finden sich statistisch deutlich weniger Ausländer im Alter von über 65 Jahren in Deutschland, obwohl sich deren Zahl in den nächsten Jahren deutlich erhöhen wird. Diese Bevölkerungsgruppe ist bisher kaum untersucht und bietet damit eine spannende Zielgruppe:

Die Lebenssituation von Migranten nach ihrer Erwerbsphase ist bislang unzureichend und in ihrer großen Heterogenität untersucht worden. [...] Sieben Prozent der ausländischen Bevölkerung sind über 65 Jahre alt - gegenüber $22 \%$ bei den Deutschen. (VON LOEFFELHOLZ 2010: Folie 3 - Vortrag vom 07.07.2010) ${ }^{27}$

Es muss davon ausgegangen werden, dass sich die Zahl in den nächsten Jahren mit steigendem Alter der ersten und zweiten Einwaderergeneration deutlich erhöht.

Deckung des zunehmenden Bedarfs durch privaten und Staatliche Träger/Akteure. (ebd. Folie 9)

Wie bereits angedeutet scheint sich bereits darzustellen, dass die intrafamiliäre Unterstützung und Versorgung in Zukunft nicht mehr gewährleistet sein kann, obwohl sie gegenwärtig meist noch intakt zu sein scheint:

Leistungen der Kinder an die Eltern werden in einem größeren Umfang erwartet als bei der deutschen Vergleichsgruppe (u.a. finanzielle Unterstuützung nach Eintritt in das Erwerbsleben; finanzielle Hilfe in Notfällen und im Alter; Hilfe im Haushalt usw.).[...]

${ }^{27}$ Eigene Quelle: Vortrag: Zur sozio-ökonomischen Situation von Migraten nach ihrer Erwerbsphase von H.D. VON LOEFFELHOLZ, BAMF: http://www.sektionaltern.de/de/praes_st_2010.htm am 07.07.2010. 
Es zeigt sich, dass ca. $6,5 \%$ der ausländischen Personen ihre Eltern/Schwiegereltern finanziell unterstützen. Bei den Deutschen liegt dieser Anteil hingegen nur bei 2\%. (ebd. Folie 11)

Leistungen der Pflegeversichung werden von Ausländern unterproportional in Anspruch genommen, es muss jedoch mit einem steigenen Bedarf in den nächsten Jahren gerechnet werden. Befriedigung des Bedarfs privat und öffentlich, wenn die jüngeren Generation nicht überfordert werden soll.

Das familiäre Verhältnis unter Migranten ist stärker ökonomisch-utilitaristisch geprägt: Integrativen Transfers kommt unter Migranten eine höhere Bedeutung zu als in deutschen Familien. (ebd. Folie 14).

Die Arbeitsmigranten sahen sich zudem oft mit dem Vorwurf konfrontiert, wegen ihreres familiär orientierten Ursprungs und selbiger Haltung, ihre Kindern nicht ausreichend genug zu fördern und dementsprechend durch ausbleibende Veränderung des sozioökonomischen Status keine Integration vornehmen würden:

In the political and academic discourses on migrant families, the intense family bonds that characterize families of Turkish origin are frequently emphasized, notions which are often viewed as familialism. Palticularly migrant families of Islamic, oriental descent were perceived as obstructing integration and participation among the social majority of their decendents. This represented a culturally tainted perspective, promoting the viewpoint that these families were often not in a position to encourage and foster the scholastic education of their children due to their low socio-economic status. In addition, one argued that traditional orientations within the families further limited their descendents' participation in and integration with the host society (RENNER 1975; MERTENS I98I).

(TEPECIK 2009: 375f)

Die Motivation, die soziale Situation zu verbessern, wurde von den Arbeitsmigranten jedoch an die Folgegenerationen weitergegeben, so dass diese sich erfolgreich zeigen konnten und damit zudem bewirkten, dass die Erstgeneration damit als erfolgreich betrachtet wurde - dieses Phänomen lässt sich ebenfalls auf die familiaren Zusammenhänge zurück führen:

The migrational project of many families who emigrated from Turkey was intended to promote their upward social mobility, ${ }^{14}$ which was not satisfactorily realized during the parent generation so that this aspiration was passed along to the next generation. In the event that second generation migrants are able to successfully establish themselves educationally, thereby achieving social progress, the parental migrational project will have qualified as a success (CIL 2000; HUMMRICH 2002).

(TEPECIK 2009: 377)

$[\ldots]$

This excerpt demonstrates how the internalization of family expectations to excel correlates with the parents' unfulfilled educational goals. The pronounced orientation alongside family needs and expectations favors the fulfillment of the family's educational aspirations.

(TEPECIK 2009: 379)

Die angeführte Familienbindung der gegenwärtig gealterten Migranten ist somit kein Hinderungsgrund, sondern fördernd für die gesellschaftliche 
Entwicklung der Migranten. Innerhalb dieser Systeme kommt es durch Rollenverschiebungen zu (psychischen) Kompensationsleitungen im Leben in der Fremde. Die zusätzliche Belastung innerhalb dieser über die Generationen weitergegebenen Ziele und Motive - an Familienmitglieder der Folgegenerationen (z.B. frühe Parentifizierung durch Übernahme von Elternrollen) - summieren sich und treten als Teilaspekt des Akkulturationsstress - dieser wird im Verlauf näher erläutert - auf:

In all cases, positive attitudes towards education are present in the families so that the children do not experience any hindrance or restriction with regard to their own education at home. Despite the fact that parents are frequently unable to fulfill scholastic placement tasks or help their children with schoolwork, they nevertheless are supportive and committed to everything relating to their children's education. In some cases, this expresses itself by the parents' exerting control and putting their children under pressure, in other cases they regularly try to motivate their children and encourage them to concentrate on school assignments. In a number of cases the older siblings stand in for their parents and help out with schoolwork and learning difficulties. All biographers demonstrate different forms of internalizing the parent's educational aspirations. Parents of Turkish origin in particular set ambitious career goals and often expect their children to pursue academic careers. They idealize specific role models from their own circle of friends or acquaintances and/or emphasize their high regard for academic occupations. As such, the children witness and experience the orientation towards and preference for certain educational or vocational goals within the family already at an early age.

(TEPECIK 2009: 380f)

\subsection{Perspektive der alten Migranten in Deutschland}

\section{Gerontologie allgemein}

„Die gerontologische Forschung beschreibt Alter(n) nicht nur als einen biologischen Prozeß, sondern als einen Prozeß, der wesentlich von sozialen, ökonomischen, ökologischen und psychischen Faktoren bestimmt ist. Das heißt, ob Alter als positive Lebensphase erlebt werden kann, ist wesentlich davon abhängig, welche materiellen, sozialen und psychischen Ressourcen den älteren Menschen zur Verfügung stehen. Alter kann in eine Sinn- und Orientierungskrise führen, in der die Frage nach „dem Gebrauchtwerden“, wenn man nicht entlohnt wird, auftaucht, und in der alte Familien- und Ehekonflikte mehr Raum bekommen." Ehepaare sind im Ruhestand plötzlich dauerhaft miteinander konfrontiert, feste Arbeitsabläufe, also Lebensinhalte, entfallen (MüLLER-WILLE 2001: 19). 
Generell gestaltet sich in vielen Fällen das Leben im Alter durch eine grundlegend geänderte Familiensituation: Die Kinder sind in der Regel selbständig, der Ehepartner ist krank oder gestorben. Dabei nimmt der Kontakt zur Familie, insbesondere der zu den Kindern, zumeist einen zentralen Stellenwert für ältere Menschen ein, der nur unzureichend durch den Kontakt zu anderen Verwandten, Freunden oder Bekannten ersetzt werden kann (DRK o.D.: 12f). Entsprechend betrachtet auch das DRK das Alter weniger als primär biologischen Prozess mit einhergehendem Verlust gewisser physischer Fähigkeiten, sondern als „in erster Linie ein soziales Schicksal“. Damit gemeint ist unsere heutige leistungsorientierte Gesellschaft, in der ältere Menschen Anerkennung verlieren, weil sie die geforderten Leistungen nicht mehr erbringen können und somit den gesellschaftlichen Bedürfnissen nicht mehr entsprechen. Insgesamt schlägt sich diese soziologische Komponente in einem von Passivität, Leistungsunfähigkeit, Krankheit und Rückzug von der Außenwelt geprägten Altersstereotyp nieder (DRK o.D.: 11).

Für Senioren gestaltet es sich also grundsätzlich problematisch, nach dem Verlust familiärer oder bekannter Bezugspersonen in einer von wirtschaftlichen Werten und dem Ideal der Jugend dominierten Welt ein Selbstbewußtsein im Alter zu entwickeln und entfalten (AWO 1996: 14).

\section{Gerontologie türkischer Migranten}

Zur Zeit ihrer Anwerbung als Arbeitskräfte in Deutschland verfügten die damals noch jungen türkischen Arbeitsmigranten - nicht zuletzt wegen der nach entsprechenden Kriterien getroffenen Auswahl - über eine vergleichsweise bessere Gesundheit als die gleichaltrige deutsche Bevölkerung. Dieser Effekt hat sich bei innen mit zunehmendem Alter jedoch deutlich abgeschwächt (DEUTSCHER BUNDESTAG 2002: 151f).

Für die hier beabsichtigten Ansätze, die Pflegedienstleistungen für ältere türkische Migranten zu verbessern, ist es unerlässlich, zunächst einen Blick auf soziale und soziologische Komponenten innerhalb dieser Bevölkerungsgruppe zu wenden.

Vielfach wird die geringe Nachfrage nach bestehenden Angeboten der Pflegedienstleister auf ein vermeintliches familiäres Netzwerk älterer türkischer 
Migranten zurückgeführt und angenommen, dass Familienangehörige die häusliche Versorgung übernehmen. Zwar entspricht es weitestgehend der derzeitigen Forschungslage, dass insbesondere türkische Familien von ihren Kindern, insbesondere Söhnen, aber auch von Verwandten derselben Generation, besonders den Geschwistern, Hilfeleistungen im Alter erwarten und tatsächlich die intragenerationellen Verwandtschaftsbeziehungen über den gesamten Lebenslauf hinweg sehr stabil sind (DEUTSCHER BUNDESTAG 2002: 152).

In der Realität zeigt sich dieses familiäre Potential aber erheblich geringer ausgeprägt als angenommen: Wenngleich der Anteil der Verheirateten unter den älteren Migranten deutlich höher und der Anteil der Verwitweten bislang deutlich niedriger ist als bei Deutschen der gleichen Altersgruppe, springt doch der hohe Männeranteil von 55\% (gegenüber $40 \%$ in der deutschen Bevölkerung) ins Auge (GRIEGER 2001: 12). Selbst bei weiterer familiärer Anbindung entfällt für allein lebende ältere türkische Migranten eine kontinuierliche Hilfe häufig, wenn die Familienangehörigen sich auf 'Pendeltouren' in der Türkei befinden (SEEBERGER 2001: 2).

Zwar leben mit $44,2 \%$ der ausländischen Senioren weniger als die vergleichbare deutsche Altersgruppe (ca. 50\%) in Ein-Generationen- und statt dessen dreimal so häufig in Mehr-Generationen-Haushalten, zu berücksichtigen bleibt aber, dass aufgrund der Anwerbesituation die Zahl ausländischer männlicher Senioren diejenige der weiblichen deutlich übersteigt (DEUTSCHER BUNDESTAG 2002: 150f). Und: „Der traditionelle Mehrgenerationenhaushalt [...] stellt sich mittlerweile auch bei den türkischen MigrantInnen als Auslaufmodell dar." (CAlicoglu et Al 2001: 93).

Dementsprechend zeigen die Erfahrungen der AWO MANNHEIM, dass ein beachtlicher Teil der türkischen Migranten der ersten Generation alleinstehend und zunehmend isoliert lebt. Sie geraten zunehmend in Einsamkeit, da sie zwischen dem Aufnahme- und dem Heimatland pendeln und sich über die Jahre von ihren Familien häufig entfremdet haben (AWO 1996: 3).

Gegenüber der gleichaltrigen deutschen Bevölkerung leiden viele ältere türkische Migranten unter erheblichen finanziellen Schwierigkeiten, da sie ursprünglich meist erst im Alter von durchschnittlich 30 Jahren nach Deutschland einreisten, wo sie zumeist als Ungelernte in den unteren Lohngruppen arbeiteten. Aus diesem Grund müssen Türken mit einer deutlich niedrigeren 
Durchschnittsrente auskommen, wobei Alleinlebende vielfach hiervon noch Familienangehörige in der Türkei mit unterhalten müssen. Sozialhilfe wird in der Regel aus Angst vor Ausweisung nicht in Anspruch genommen (AWO 1996: 4).

BÜKRÜCÜ weist in diesem Zusammenhang auf die spezifische Lage im Ruhrgebiet hin, wo etliche türkische Migranten als Bergleute gearbeitet haben und aufgrund der starken Arbeitsbelastung statt mit 60 bis 65 Jahren bereits mit 50 bis 55 Jahren arbeitsunfähig geworden und vorzeitig in Rente gegangen sind. Somit stehen innen auch weniger finanzielle Mittel zur Verfügung als bei Berufstätigkeit bis zum Erreichen des Rentenalters (BÜKRÜCÜ 2001: 30).

Nicht unerwähnt bleiben sollten an dieser Stelle die Erfahrungen MuSTAFA CALICOglu ET AL's im Rahmen des Modellprojektes „Multikulturelle Senioren- und Familienarbeit" im Bochumer Stadtteilzentrum Bochum Dahlhausen. Der Verein betreut ältere türkische Migranten u.a. durch Hilfestellungen im Umgang mit den jeweiligen Rentenversicherungsträgern, wobei sich wiederholt schwerwiegende Probleme im Rentenversicherungsverlauf aufgrund der vorhandenen Lücken auftun. Als ein Grund muss sicherlich erachtet werden, dass viele Migranten amtliche Dokumente wegen der Sprachschwierigkeiten und einem niedrigen Bildungsstand in ihrer Wichtigkeit nicht erkannt und darum Schreiben von Versicherungsanstalten, Arbeitsverträge, Lohnabrechnungen, Urlaubsscheine etc. nicht aufgehoben haben. Vielfach sind ihnen aus Unkenntnis auch Anrechnungszeiten verloren gegangen, wenn sie z.B. für „Sonderurlaube“ in der Heimat statt der vermeintlichen Urlaubsscheine tatsächlich wiederholt zwischenzeitliche Kündigungen unterschrieben ohne festzustellen, dass es sich bei der anschließenden Wiederaufnahme der Arbeit tatsächlich um eine Wiedereinstellung handelte. Schließlich mussten die Mitglieder des Vereins bedauernd feststellen, dass auch das Arbeitsamt vielfach mitnichten seiner Informationspflicht Genüge getan hatte: Vielmehr unterbreitete es arbeitslosen türkischen Migranten zum 60. Lebensjahr ein vermeintlich verlockendes Angebot, dem viele der ortsansässigen Betroffenen zustimmten: Die Migranten könnten bis zu 17 statt den bisher 3 Wochen Urlaub erhalten und anschließend in Frührente gehen. Nicht aufgeklärt wurden die Migranten allerdings über die erheblichen finanziellen Folgen dieses Schritts, der erhebliche Rentenkürzungen nach sich zog (CAlicoglu ET AL 2001: 97f). So verwundert die Beobachtung kaum, dass in diesem Stadtteil etliche türkische Senioren mit einer Rente unterhalb des 
Sozialhilfesatzes auskommen müssen (EBD.: 101).

Als mit ursächlich für den schlechteren Gesundheitszustand älterer türkischer Migranten gegenüber der gleichaltrigen deutschen Bevölkerung gilt im übrigen vielfach der Heimkehrwunsch mit einhergehenden Einschränkungen an sich: Überall sparten sie für die Rückkehr, sei es an der Wohnung, der Ernährung oder Bekleidung (BÜKRÜCÜ 2001: 25f). So ergab beispielsweise eine im Jahr 1993 vom Statistischen Bundesamt durchgeführte 1-\%-Stichprobe der Wohnraumsituation, dass Deutschen im Schnitt pro Kopf 39qm Wohnraum zur Verfügung standen, Türken dagegen nur 17,9qm (vgl. HAMBURGER BEHÖRDE FÜR Arbeit, Gesundheit und SozIALES 1998: 14). Mehrarbeit und -belastung leisteten hierzu ein Übriges. Zusätzlich litten die Türken unter psychischen Problemen, insbesondere der kontinuierlichen Angst, abgeschoben zu werden. Für ältere türkische Frauen kommt als weiterer Aspekt die Vereinsamung hinzu - anders als ihren Männern steht ihnen aufgrund der Geschlechterrollen beispielsweise kein Café-Häuschen für Kontakte zur Verfügung, häufig fühlen sie sich auch durch die Erziehung der Enkelkinder überfordert. Ärztlichen Angaben zufolge hat jede dritte ältere türkische Frau Psychopharmaka im Haus (BüKRÜcÜ 2001: 25f).

Der deutsche Bundestag nennt in seinem Bericht zu Ausländerfragen als weiteren gesundheitsbeeinträchtigenden Faktor den durch den Migrantenstatus ausgelösten Stress, der sich im Alter in Krankheiten manifestieren könne (DT. BUNDESTAG 2002: 152) ${ }^{28}$.

Darunter fallen unter anderem Rollenkonflikte innerhalb der Ehe oder Familie zwischen der traditionellen Geschlechterrolle als Mann oder Frau in der Türkei und dem Rollenverständnis innerhalb der deutschen Gesellschaft (SCHMELING-KLUDAS ET AL 2002: 201).

Einem anhand einer Befragung älterer türkischer Migranten erstellten Forschungsbericht des Bundesministeriums für Arbeit und Sozialordnung vom August 1992 war zu entnehmen, dass zu diesem Zeitpunkt 54\% von ihnen unter körperlichen oder seelischen Beschwerden litten. Herausragend waren darunter Verschleißerscheinungen des Stütz- und Bewegungsapparates (48\% mit Rückenoder Gliederschmerzen), 31\% von innen wiesen Gehbeschwerden, schwere

28 Hinweise auf die gesundheitsbelastenden Auswirkungen negativer, plötzlich auftretender und einschneidender Lebensereignisse oder Verluste, die sich „im Alter als Krankheiten manifestieren können" bietet die Stressforschung bereits seit geraumer Zeit (vgl. GRIEGER 2001: 16). 
chronische Erkrankungen, Herz- und Kreislaufbeschwerden sowie psychosomatische Krankheitsbilder auf. Auffällig war auch das - im Vergleich zur deutschen Bevölkerung - besonders häufige Auftreten dieser Erkrankungen bei älteren, nichtberufstätigen türkischen Frauen (TUFAN 1999: 51).

Bestätigt und ergänzt werden diese Angaben durch CALICOGLU ET AL für den Stadtteil Bochum/Dahlhausen. Sie führt zusätzlich Erkrankungen der Atmungsorgane, Stoffwechselerkrankungen, Magen- und Darmgeschwüre sowie Depressionen auf ebenso wie Hintergründe migrationsbedingter besonderer psycho-sozialer Belastungen, als da sind: Heimweh, viele Überstunden bei körperlicher Schwerstarbeit, kulturell bedingte soziale Isolation und sprachliche Schwierigkeiten (CALICOGLU ET AL 2001: 98).

Unter den $91 \%$ der türkischen Klientel der ersten Migrantengeneration einer monokulturellen psychosomatischen Therapiestation in Gießen lag die Geschlechterverteilung beispielsweise bei 65\% Frauen und 35\% Männern (RODEWIG 2002: 208f).

SLUZKI liefert als Grund für diese stärkere Belastung die gerade aus ländlichen Regionen stammenden Migranten betreffende Rollenverteilung der Geschlechter, in der klassisch der Mann zu Arbeit geht und durch die Außenorientierung eine individuelle Autonomie und gewisse Selbstsicherheit erlangt. Der innenorientierte Partner erfährt dagegen zunehmende Isolation - teils auch bedingt durch die Autonomisierung des anderen - „was krisenhaft eskalieren kann." (SLUZKI 2001: 109).

Aus einer Analyse von 184 aus der stationären Rehabilitation entlassenen türkischen Migranten der Segeberger Kliniken $\mathrm{GmbH}$ ging hervor, dass $62 \%$ von ihnen von somatoformen Störungen betroffen waren. Mehr als ein Viertel der Patienten litt unter anhaltender somatoformer Schmerzstörung, an zweiter Stelle folgten depressive Störungen einschließlich der Dysthymie mit 37\% (ScHMELINGKLUDAS ET AL 2002: 196).

Diese gesundheitliche Einschätzung bekräftigt TACKE-BORCHERT mit ihren Schlussfolgerungen zu einer Analyse der Arbeitsunfähigkeitsgutachten in Frankfurt/Main im Jahr 2000. Obwohl sich aus den Akten nur anhand der Namen der Versicherten rekonstruieren ließ, ob es sich bei innen um Ausländer oder Deutsche handelte, ergab sich dennoch ein beachtlicher Anteil von insgesamt $45,8 \%$ nichtdeutschen Versicherten. Jedoch hatten Ausländer in der Stadt 
Frankfurt allgemein an der Gesamtbevölkerung der 50- bis unter 65-Jährigen nur einen Anteil von 24,9\%. Trotz dieser vagen Datenlage schloss die Autorin daraus, die überproportional häufige Begutachtung nichtdeutscher Versicherter könne auf ihren höheren Krankenstand hinweisen (TACKE-BORCHERT 2001: 41f).

Zwar sind bei türkischen Migranten im fünften Lebensjahrzehnt - ebenso wie bei Deutschen - die Rehabilitations-Intensitäten am höchsten, werden aber im Vergleich zu den deutschen Versicherten deutlich seltener beansprucht ${ }^{29}$. Auffällig hinsichtlich der türkischen Rehabilitanden ist zudem, dass sie insbesondere bei den psychischen Erkrankungen die höchste Nutzungsintensität zeigen, wohingegen sie in der klassischen Diagnosegruppe „Skelett, Muskeln, Bindegewebe“ die Rehabilitationsmöglichkeiten deutlich und auffällig seltener in Anspruch nehmen als andere ausländische Bevölkerungsgruppen (HACKHAUSEN 2001: 20).

Studien aus Mannheim haben ergeben, dass die überwiegende Mehrheit der Türkinnen und Türken während ihres Arbeitslebens an keinen Rehabilitationsmaßnahmen teilnahmen, da sie befürchteten, im Falle einer Rückkehr in die Türkei ihre Ansprüche auf die geleisteten Versicherungsbeiträge zu verlieren (AWO 1996: 3).

Zusammenfassend läßt sich also geradezu zwangsläufig schlußfolgern, dass pflegebedürftige Senioren aus der ersten Migrationsphase noch deutlich jünger als heute pflegebedürftige deutsche Senioren sind - unter innen befinden sich noch keine Hochbetagten. Verantwortlich hierfür sind die vergleichsweise hohen Belastungsfaktoren, denen sie in ihrem Alltag in Deutschland unterlagen und meist weiterhin unterliegen (MÜLLER-WILLE 2001: 21).

MüLLER-WILLE vermutet als weitere Ursache für die fehlende Nachfrage älterer türkischer Migranten nach bestehenden Angeboten der Altenhilfe ihre Wahrnehmung von alten Menschen in Deutschland, die den eigenen Vorstellungen diametral entgegenstünden: Ein verbreitetes Bild für ältere türkische Migranten seien von ihren Kindern allein gelassene alte deutsche Menschen, die bestenfalls von innen finanziell unterstützt würden und ansonsten nahezu ausnahmslos ihren letzten Lebensabschnitt verlassen in einem Altenpflegeheim

29 Seit Beginn der 90er Jahre deuten höhere Nutzerzahlen allerdings auf eine steigende Inanspruchnahme dieser Leistungen hin, die dennoch weit unter denjenigen ihrer deutschen Altersgenossen liegen (HACKHAUSEN 2001: 20). 
verbrächten. Die Vorstellung, selbst die letzten Jahre isoliert in einer solchen Einrichtung zubringen zu müssen, die dortige Sprache weder zu verstehen noch selbst verstanden zu werden, ohne den eigenen Lebensvorstellungen und religiösen Lebensbezügen nachgehen zu können, stelle sich für viele ältere Türken als beängstigend dar (MüLLER-WILLE 2001: 25).

Identische Erfahrungen berichten CALICOGLU ET AL aus Bochum/Dahlhausen: „Die schlimmste Befürchtung betrifft die Vorstellung, in einem deutschen Altersheim untergebracht zu werden und dort ihrer kulturellen und religiösen Gewohnheiten beraubt zu werden." (CALICOGLU ET AL 2001: 100).

Nicht bestätigt findet sich diese Einschätzung in einem Erfahrungsbericht BRAUN ET AL's anlässlich der Informationsveranstaltung zur Erprobung des DRKMedienkoffers in Stuttgart im Jahr 2001. Dort war die Unterbringung in einem Alten- bzw. Altenpflegeheim zwar zentrales Anliegen der türkischen Teilnehmer, Besorgnis äußerten sie aber vor allem hinsichtlich der Finanzierbarkeit einer solchen Unterbringung. Ansonsten wurden Altenpflegeheimen durchaus positive Aspekte abgewonnen, nämlich in solchen Einrichtungen auch als alter und kranker Mensch mit anderen Menschen zusammen zu sein und gemeinsam essen zu können. Vielfach wurde dort sogar der Wunsch nach entsprechenden Wohnformen speziell für ältere türkische Menschen geäußert (BRAUN ET AL 2001: 81).

Bemerkenswert in diesem Zusammenhang ist die Erfahrung AL-BARGHOUTIS während der Erprobung des DRK-Medienkoffers in Reutlingen (mit einer türkischen Frauen- sowie einer Männergruppe) und Tübingen (mit einer gemischtgeschlechtlichen, zumeist aus Ehepaaren zusammengesetzten Gruppe) älterer türkischer Migranten. Entgegen der fast generell vermuteten hohen Erwartungshaltung, die Familienmitglieder hätten für die Pflege zu sorgen, erfuhr sie sowohl aus der Frauen- als auch der gemischtgeschlechtlichen Gruppe den großen Wunsch, „auch im hohen Alter ihr Leben unabhängig von den Kindern meistern zu wollen.“ (AL-BARGHOUTI 2001: 71).

Berücksichtigt werden sollte an dieser Stelle allerdings auch MüLLER-WILLES Hinweis, dass bei der Auseinandersetzung mit dem Älterwerden vor dem Hintergrund der Migration nicht nur die informative, sondern auch die emotionale Ebene nicht vernachlässigt werden dürfe. Sie bezieht sich hier vor allem auf das 
aufgegebene ursprüngliche Lebensziel, in die Heimat zurückzukehren und die damit verbundenen Mühen, Benachteiligungen und Belastungen der Migration mit ihren physischen und psychischen Auswirkungen in Kauf zu nehmen. Mit der Aufgabe der einstigen Pläne geraten etliche ältere Migranten somit im Lebensrückblick in ein Dilemma (MÜLLER-WILLE 2001: 22f).

\section{NIZW und Übertragung auf Deutschland}

Die unverändert fehlenden Mitspracherechte (älterer) türkischer Migranten selbst auf lokalpolitischer Ebene zeigen sich unter anderem an der Tatsache, dass die deutsche Sozialplanung und -politik prinzipiell noch immer unzureichend auf die Bedürfnisse älterer und alter Migranten eingestellt ist (FREWER/JÄGER 2001: 39).

Schon 1998 stellte HINZ-RommEL bereits bedauernd fest, dass zwar die Zahl der Migranten in Deutschland kontinuierlich ansteige, jedoch keineswegs den Eindruck vermittelt werde, die Einrichtungen der Altenhilfe reagierten auf diese neue Herausforderung (HINZ-ROMmEL 1998: 36).

Obwohl also die deutsche Gesellschaft zweifelsohne multikulturell geprägt ist, gestaltet sich das hiesige Gesundheitssystem bislang fast ausschließlich monokulturell (HILL/RÖHL 2002: 115) - WESSEL-NEB spricht gar von einer "strukturellen und institutionellen Diskriminierung der Migrantinnen und Migranten“ im Öffentlichen Gesundheitsdienst (WESSEL-NEB 2002: 125).

Unterstrichen werden diese Einschätzungen durch einhellige Berichte nach denen, die Nachfrage älterer Migranten nach bestehenden Angeboten im Öffentlichen Gesundheitswesen fehlten nahezu gänzlich.

Zwar gibt es keine übergreifende statistische Erfassung, wie viele bzw. wenige ältere Migranten die Dienste der Altenhilfe in Anspruch nehmen, punktuellen Studien zufolge liegt aber der Anteil ausländischer Bewohner und Bewohnerinnen in der stationären Altenhilfe in den letzten Jahren zwischen 1,5 und 1,7 \% (FREWER/JÄGER 2001: 40).

Von deutscher Seite her wird hieraus fälschlicherweise vielfach ein geringer Bedarf an Unterstützungsleistungen gefolgert, ohne die spezifischen Belastungen und Bedürfnisse der Migranten wahrzunehmen (FREWER/JäGER 2001: 40).

So ist in diesem Zusammenhang nach wie vor die oben bereits erwähnte 
und anhand statistischer Daten widerlegte These nicht ausgestorben, Migranten fänden bei Familie, Freunden und Bekannten, meist derselben Nationalität, die benötigte Hilfe und Unterstützung bei Krankheit und/oder Pflegebedürftigkeit, verfügten also über ein soziales Netzwerk, dass die Inanspruchnahme stationärer Pflegeeinrichtungen überflüssig mache (s. Z.B. STANJEK 2002: 271).

Symptomatisch ist auch GRIEGERS vorwurfsvoll anmutender Hinweis, Migranten nutzten ,jenseits von freiwilliger oder erzwungener Begutachtung [...] das öffentliche Gesundheitswesen [...] zu wenig von sich aus". Allerdings hebt sie an gleicher Stelle hervor, dass das Öffentliche Gesundheitswesen seinerseits in der Regel eine große Distanz zu Migranten aufweise und diese bis in die Gegenwart häufig noch nicht einmal als Klienten im Blick habe (GRIEGER [a] 2001: 18).

Von pflegebedürftigen Migranten wird erwartet, sich selbständig an die entsprechenden Einrichtungen zu wenden, die schließlich allen offen stünden (FREWER/JÄGER 2001: 40).

Eine entsprechende Erfahrung schildert HINZ-ROMMEL nach einem persönlichen Besuch der Sozialstation in Berlin, damals einem Quartier mit fast $40 \%$ Ausländeranteil, der seinerzeit zu weniger als 10\% von Migranten aufgesucht wurde. Auf verwunderte Nachfrage an die Mitarbeiter nach dieser geringen Nutzung der Einrichtung erhielt er lediglich die Antwort, die Tür sei schließlich nicht verschlossen (HINZ-ROMMEL, 1998: 36).

Verantwortlich hierfür sind die in Deutschland ausgeprägten KommStrukturen zum Erhalt von Informationen, welche älteren türkischen Migranten als eine der etlichen Zugangsbarrieren die Beanspruchung von Altenhilfe erheblich erschweren. Die bestehenden Sprach- und Verständigungsprobleme behindern sie darin, selbständig Informationen über die bestehenden Angebote, Dienstleistungen und Einrichtungen der Altenhilfe zu erwerben (MüLLER-WILLE 2001, S. 28).

Etliche der älteren türkischen Migranten aus den frühen Anwerberstaaten sprechen auch nach jahrzehntelangem Aufenthalt in der Bundesrepublik nur ein vergleichsweise schlechtes Deutsch - oft haben die Männer gerade die notwendigsten Deutschkenntnisse für den Arbeitsplatz erworben. Dies trifft in besonderem Maße auf Frauen zu. Noch schlechter wiederum stellt sich die Lage für beide Geschlechter im Umgang mit der Schriftsprache dar (HAMBURGER 
BEHÖRDE FÜR ARBEIT, GESUNDHEIT UND SOZIALES 1998: 15).

Beispielhaft sind hierfür die Erfahrungen RODEWIGs zur mangelnden deutschen Sprach- und Schriftsprachenkompetenz für die monokulturelle türkischsprachige Therapiestation in Gießen, auf welcher $80 \%$ der türkischen Patienten (zum weitaus größten Teil aus der ersten Migrantengeneration) die Schule entweder gar nicht oder weniger als fünf Jahre besucht hatten. Nach Therapeuteneinschätzungen waren bei 2/3 der Männer und Frauen die deutschen Sprachkenntnisse nur ungenügend oder mäßig vorhanden, deutlich schlechter stellte sich die Situation bei der schriftlichen Sprachkompetenz dar. Frauen verfügten überdies über eine deutlich geringere Sprachkompetenz als Männer (RODEWIG 2002: 208f) $^{30 .}$

Ausgerechnet die erste Migrantengeneration, also die Seniorengruppe, die der Inanspruchnahme vorhandener Pflegedienstleistungen akut bedürfte, weist eine alarmierend hohe Quote an Analphabetismus auf. So berichtet BÜKRÜCÜ, 70 bis $80 \%$ der PatientInnen seines ambulanten Pflegedienstes in Gelsenkirchen seien AnalphabetInnen (BÜKRÜCÜ 2001, S. 35) ${ }^{31}$.

Da ältere türkische Migranten über kaum oder keine Kontakte zur deutschen Wohnbevölkerung verfügen, fehlen ihnen auch eventuelle Hilfskräfte, welche als Multiplikatoren für weitere Hilfsangebote dienen könnten (SEEBERGER 2001: 3).

So verwundert es kaum, dass das unter Senioren allgemein hohe Informationsdefizit über das Leben im Alter im besonderen Maße für die älteren türkischen Migranten zutrifft. Beispielhaft steht hierfür die Gründung eines

30 Dass ein grosser Bedarf nach Sprach- und Alphabetisierungskursen besteht, belegt das Beispiel aus Bochum/ Dahlhausen, wo der Verein IFAK e.V. im Rahmen der multikulturellen Seniorenarbeit überraschend hohe Resonanz vor allem älterer türkischer Migrantinnen auf die angebotenen Sprachkurse erfuhr (CALICOGLU et al 2001: 103f).

31 Das Sprach- respektive Kommunikationsproblem läßt sich hierzulande wohl auch nicht ad hoc lösen - anders als in den Niederlanden, wo es (Staatssekretär JORDAN durchaus bekannt) „umfassende staatlich finanzierte Lösungen des Sprachenproblems gibt“ (JORDAN 2000: 22), sind in der Bundesrepublik offenbar keine Maßnahmen zur Behebung der grundlegendsten Kommunikationshemmnisse angedacht. Blumig vermerkt der Dt. Bundestag in seinem jüngsten Bericht lediglich, daß „eine Reihe von Zuwanderergruppen mit dauerhaftem Aufenthalt [...] nicht in die Sprachförderung einbezogen werden (können), da sich die derzeitigen Förderansprüche nicht an dem jeweiligen Integrationsbedarf orientieren, sondern sich aus dem rechtlichen Status bzw. den Vorbeschäftigungs-zeiten ableiten...“. Und behauptet darüber hinaus, daß „obwohl der begrenzte Mittelrahmen für das Gesamtmodell im Verhältnis zu den bestehenden Angeboten quantitative Reduzierungen erforderlich macht, ..., das Konzept [...] nicht automatisch auch zu einem qualitativen Niveauverlust und damit reduzierter Sprachkom-petenz führen muß..." (DEUTSCHER BUNDESTAG 2001: 111). Vergegenwärtigt man sich, dass ausreichende deutsche Sprach- und Schriftkenntnisse eine der Voraussetzungen zum Erwerb der deutschen Staatsangehörigkeit sind (vgl. SANTEL/WEBER 2000: 126), muten diese Sätze geradezu zynisch an. 
Seniorenbüro International in der Stadt Essen, wo Migranten praktisch keine Kenntnisse über die Pflegeversicherung aufwiesen:

- etliche Migranten wussten nicht, dass sie als Arbeitnehmer Pflichtmitglied der Pflegeversicherung sind,

- die Möglichkeiten von Hilfsmitteln waren vielfach unbekannt,

- für Migranten standen keine muttersprachlichen Informationsschriften seitens der Kranken- und Pflegekassen zur Verfügung (YILMAZ 2001: 11ff)

BÜKRÜCÜ hält das Fehlen der Informationsmittel zur Pflegeversicherung seitens der Bundesregierung für durchaus gewollt: „Ihr macht Broschüren, Ihr klärt die Leute auf über Renten usw. Wieso habt Ihr die schönen bunten Broschüren nicht auch für die Pflegeversicherung? Aus einem einfachen Grund: Je mehr sie aufklären würden, desto mehr müssen sie ja bezahlen. [...] Wenn wir einen ganz normalen deutschen Patienten aufnehmen, dann sind die Patienten bereits wunderbar aufgeklärt. [...] Wenn wir türkische Patienten aufnehmen müssen wir sie wirklich von Null aufnehmen [...] [und] ihnen erklären, was Pflegeversicherung überhaupt bedeutet." (BÜKRÜCÜ 2001: 29f).

$\mathrm{Zu}$ den sprachlichen Barrieren gesellen sich des Weiteren die kulturellen in der Weise, dass die bestehenden Angebote der Altenhilfe nicht nur auf die türkischen Senioren nicht eingestellt, sondern in der Regel für sie auch ungeeignet sind (dabei handelt es sich zumeist um Angebote von Vereinen, Wohlfahrtsverbänden, Kirchenkreisen etc.) (CALICOGLU ET AL 2001: 95).

Infolgedessen konzentrieren sich die auf Gesundheitsversorgung bezogenen Kontakte der Migranten in der Regel einerseits auf Einrichtungen der eigenen Nationalität und andererseits internationale Zentren. Weit verbreiten sind die aufgrund negativer Erfahrungen oder Erwartungen zusätzlichen Hemmungen, deutsche Institutionen aufzusuchen, wozu sich die Angst vor ausländerrechtlichen Konsequenzen bei Inanspruchnahme von Sozialleistungen gesellt (MüLLER-WILLE 2001, S. 28).

Die Defizite deutscher Institutionen, wie sie YILMAZ für Essen auflistet, können demnach als symptomatisch betrachtet werden:

- unter den etwa 1500 Kunden für Inkontinenzmittel eines großen ansässigen Sanitätshauses befand sich kein einziger Migrant, 
- die Angebote der städtischen "Offenen Altenhilfe" konzentrieren sich ausschließlich auf deutsche Senioren,

- deutsche Institutionen halten kaum Informationsangebote in den jeweiligen Landessprachen bereit,

- der Anteil ausländischer Mieter bei Trägern von Seniorenwohnungen und des betreuten Wohnens bewegt sich im Promillebereich,

- für die altersrelevanten Bereiche, insbesondere die Pflegeversicherung, steht keinerlei muttersprachliches Informationsmaterial zur Verfügung, sieht man von zwei türkisch-sprachigen Broschüren der Bundesknappschaft $a b^{32}$,

- Mitarbeiter von Kranken- und Pflegekassen, Gesundheitsämtern, Wohnungsgesellschaften, Pflegediensten und Krankenhäusern sind mit den spezifischen kulturellen Besonderheiten der Migranten unterschiedlicher Herkunft nicht vertraut,

- Kranken- und Pflegeschulen berücksichtigen in der Ausbildung nicht die Besonderheiten der Pflege bedürftiger Migranten,

- muttersprachliche Pflegeberatung fehlt im Angebot nahezu vollständig (YILMAZ 2001: 15)

Beispielhaft für den letztgenannten Punkt steht die länderübergreifenden Akademie für öffentliches Gesundheitswesen in Düsseldorf ${ }^{33}$, die Beschäftigten im öffentlichen Gesundheitswesen Möglichkeiten zur qualifizierten Aus- und Weiterbildung anbietet, da weder in Fachreferaten, Fortbildungsveranstaltungen oder Lehrgängen das Thema der älteren Migranten behandelt wird (DIE AKADEMIE FÜR ÖFFENTLICHES GESUNDHEITSWESEN IN DÜSSELDORF 2001: 75f).

Ältere türkische Migranten haben also ohne muttersprachliche Beratung oder Informationsmaterialien in türkischer Sprache praktisch keinen Zugang zu den notwendigen Informationen für die Inanspruchnahme der innen zustehenden Leistungen.

Wesentlich fortschrittlicher erscheint demgegenüber die Wahrnehmung älterer Migranten und ihrer Bedürfnisse in anderen europäischen Ländern. Vorbildlich präsentieren sich hier beispielsweise die Niederlande, wo schon länger eine fundierte Auseinandersetzung mit der Problematik der älteren Migranten stattfindet. So plädiert die dortige Stiftung für interkulturelle Gesundheitsfürsorge,

32 BÜKRÜCÜ widerspricht dieser Aussage, nachdem er die Bundesknappschaft persönlich um die bestehenden Materialien gebeten hat. Ihm zufolge gibt die Institution zwar „schöne bunte Kataloge oder Prospekte" zur Rente und Krankenkassen auch in türkischer Sprache heraus. Es fehlen aber vergleichbare Materialien für die Pflegeversicherung. Er vermutet als Grund die Befürchtung von Mehrkosten bei verbesserter Aufklärung (BÜKRÜCÜ 2001, S. 29).

33 Träger sind die Länder Bremen, Hamburg, Hessen, Niedersachsen, Nordrhein-Westfalen und Schleswig-Holstein (DiE AKADEMIE FüR ÖFFENTLICHES GESUNDHEITSWESEN IN DÜSSELDORF 2001:75). 
die sich schwerpunktmäßig mit psychiatrischen Belangen auseinandersetzt, für eine „Interkulturalisierung“ bei der Betreuung von Migranten und Flüchtlingen. Die Stiftung fordert hiermit einen Kurswechsel dahingehend, dass die geistige Gesundheitsfürsorge sich einer multikulturellen Umgebung anzupassen habe, anstatt Migranten und Flüchtlinge in das bestehende Gesundheitssystem einzugliedern. Aus einer kulturkritischen Sichtweise heraus gehen die Stiftungsmitglieder davon aus, die Gesundheitsfürsorge sei von den vorherrschenden kulturellen niederländischen Werten durchdrungen und agiere gegenüber den Migranten paternalistisch (VAN DIJK ET AL O.D.).

Dass derartige Thesen keineswegs als Theorie in wissenschaftlichen Publikationen verstauben, demonstriert das Informationsprogramm „Ouder worden in Nederland“ (Älter werden in den Niederlanden) des Niederländischen Instituts für Gesundheitsfürsorge und Gemeinwohl (Nederlands Instituut voor Zorg en Welzijn/NIZW), welches für ältere und behinderte Menschen, Migranten und Obdachlose eine Palette verschiedener Aufgaben im Bereich der Gesundheitsfürund -vorsorge bearbeitet.

Dazu gehört auch eine Informationsreihe für ältere Migranten über Struktur und Fürsorgemöglichkeiten an ihrem Wohnort, die darauf abzielt

- ausländischen Senioren Informationen über Angebote der Altenhilfe an ihrem Wohnort zu vermitteln,

- durch Gespräche und Informationen die Bereitschaft der ausländischen Senioren zu wecken, bei Bedarf auch institutionelle Hilfen anzunehmen,

- zu erfragen, welche spezifischen Besonderheiten Institutionen der Altenhilfe berücksichtigen müssten, um von ausländischen Senioren in Anspruch genommen zu werden. (FACH 2001: 33f).

Die sehr positiven Erfahrungen im Umgang mit diesem Informationsmodul seit 1994, an dem sich mehr als 100 Gruppen beteiligt haben, bewogen das DRK, welches sich bereits seit 1989 kontinuierlich mit der Thematik auseinandersetzt, Mitte der 90er Jahre (DRK 2002: 12f) das Informationsmodul auf Bundesverbandsebene in Zusammenarbeit mit dem NIZW auf Deutschland zu übertragen. In diesem Sinne sollte in enger Kooperation der Fachgebiete Migrationsarbeit und Altenhilfe zielgruppengerechtes Informationsmaterial erarbeitet, deutschen Pflegekräften interkulturelle Kompetenz als Schlüsselqualifikation vermittelt und Altenhilfeangebote für alte Migranten geöffnet 
und angepasst werden (FACH 2001: 34ff). Für den Transfer des Moduls auf die deutschen Gegebenheiten beriefen die Organisationen bewusst eine interdisziplinäre und trägerübergreifende Projektgruppe ein ${ }^{34}$. Die dahinter stehende Absicht, durch Zusammenfügung möglichst unterschiedlicher Arbeitsebenen und -bereiche sowie Nutzung von Synergieeffekten (FACH 2001: 36) trägt bislang insofern Früchte, als nicht nur die hier dargestellte Problematik älterer ausländischer Migranten, sondern auch die aus obigen Ansätzen entstandene Informationsreihe „Älter werden in Deutschland“ vergleichsweise ausführliche Berücksichtigung im Bericht der Ausländerbeauftragten gefunden haben. Dort findet sich darüber hinaus die Forderung nach einer kultursensiblen Altenpflege, wofür sich die Ausländerbeauftrage als Mitglied einer bundesweiten Arbeitsgruppe, welche im Mai 2002 ein „Memorandum für kultursensible Altenhilfe“ fertiggestellt hat, ebenfalls engagierte. Entsprechend fließen die Konzepte der Informationsreihe auch in die abschließenden Empfehlungen ein (DEUTSCHER BUNDESTAG 2002: 150ff).

Damit scheint der durch die Konzeption der Projektgruppe bezweckte wechselseitige Prozess einzusetzen, nämlich daß auch deutsche Institutionen von den Erfahrungen hinsichtlich „[...] praktische[r] Durchführung, Auswertung und Erweiterung entscheidende Rückschlüsse über den konkreten Bedarf der älteren Migranten ziehen können“ (FACH 2001: 39).

Das Informationsmaterial unter dem Motto „Älter werden in Deutschland“ wurde eingangs nur für Senioren türkischer Herkunft vorbereitet, da sie die größte Gruppe ausländischer Bevölkerung in Deutschland stellen, und zunächst in einzelnen Arbeitsexemplaren an den Probestandorten DRK/Berlin, AWO/Bremen und später der Kommune Bonn erprobt (FACH 2001: 37ff).

Das Ziel war, einen Perspektivwechsel von der bisherigen Komm-Struktur zu einer Geh-Struktur zu verwirklichen, indem die Bedürfnisse der Migranten direkt und praktikabel anvisiert und aktiv auf die Senioren zugegangen wurde, ohne sie intellektuell zu überfordern. Gleichzeitig bemühten sich die Teilnehmer des Projektes um Vermeidung paternalistischer Tendenzen, indem „Migranten nicht als passive Opfer gesehen [wurden], sondern als Pioniere, die den Mut hatten, ihr

34 Dem Projekt gehören mittlerweile folgende Institutionen fest an: NIZW, Netherlands Institute for Care and Welfare/Utrecht, DRK, AWO Bundesverband und Kreisverband Osnabrück, Amt für soziale Angelegenheiten und Altenarbeit/Heidelberg, Altenhilfe/Bonn, Beauftragte der Bundesregierung für Ausländerfragen, Gesundheitsamt des Landratsamtes Rhein-Neckar-Kreis, Referat für Multikulturelles/Bonn (FACH 2001:36f). 
eigenes Land zu verlassen und für ein besseres Leben für sich und ihre Kinder zu kämpfen." In diesem Sinne sollten die praktischen Informationen auch lediglich zur Erweiterung des Verhaltensrepertoires der türkischen Senioren dienen, dass ihnen gleichzeitig weiterhin „ethnische Nischen“ einräumte - angestrebt wurde nicht etwa eine „Zwangsintegration“, sondern das Aufzeigen von Wegen für ergänzende Hilfe im Alter (FACH 2001: 41).

Die Übertragung des niederländischen Modells auf Deutschland sollte bei türkischen Senioren einen dreistufigen Prozeß anstoßen, nämlich erstens, die emotionalen Barrieren zu überwinden, die durch Erwartungshaltung gegenüber Familienangehörigen bislang einer Inanspruchnahme bestehender Angebote im Wege standen. Anschließend sollten die Senioren mittels Gesprächen, dem Einsatz von Bildern und Filmen sowie Exkursionen ${ }^{35}$ Informationen über Hilfsangebote am eigenen Wohnort erhalten. Schließlich waren die eigenen Wünsche der älteren Migranten hinsichtlich der Aktivitäten oder Dienstleistungen von Interesse, insbesondere, was in ihren Augen gegebenenfalls am bestehenden Angebot verändert bzw. welche Elemente zusätzlich mit aufgenommen werden müßten (AL-BARGHOUTI 2001: 44f).

Ein ähnliches Modell verfolgte auch die ARBEITERWOHLFAHRT (AWO) zwischen 1993 und 1996 mit dem Projekt zum „Aufbau einer Altentagesstätte für türkische Seniorinnen und Senioren“ in Mannheim. Zielgruppe waren türkische Langzeitarbeitslose und Rentner ab 50 Jahre (vgl. AWO 1996: 3). Positiv erscheinen die Dokumentationen etlicher weiterer, ähnlich gelagerter Projekte wie den o.g. Seniorenbüro International in Essen oder die Bemühungen um eine „Offene multikulturelle Seniorenarbeit“ in Bochum/Dahlhausen ${ }^{36}$.

Ebenso muss die Gründung der „Expertengruppe Transkulturelle Pflege“ 1998 in Berlin, einer Arbeitsgemeinschaft aus Pflegekräften, LehrerInnen für Pflegeberufe, Pflegedienstleistungen, HochschullehrerInnen und StudentInnen, als Resultat der bundesweit einsetzenden Auseinandersetzung mit den Problematiken der älter werdenden Migranten betrachtet werden. Generelles Ziel dieser Arbeitsgemeinschaft ist die Integration der „Transkulturellen Pflege“ in das Sozial-

35 Mit dem Einsatz vieler Medien sollte gewährleistet werden, dass Analphabeten gleichermaßen von den Veranstaltungen profitierten (FACH 2001: 41).

36 Für das Bochumer Projekt bleibt besonders hervorzuheben, dass der multikulturelle Ansatz, nämlich gemeinsame ökumenische (zwischen Moschee und evangelischer Kirche) und soziale Angebote für sowohl türkische wie auch deutsche Senioren anzubieten, auf positive Resonanz zu stoßen scheint (vgl. Calicoglu et al 2001: 108ff). 
und Gesundheitswesen sowie eine interdisziplinäre Vernetzung von Praxis und Forschung anhand der Schwerpunktthemen „Aus-, Fort- und Weiterbildung“ und „Pflegemanagement“. Angelehnt an den Orientierungspunkten des Kultursministeriums aus dem Jahre 1996 gehörten zu den angestrebten Kriterien für interkulturelle Kompetenz:

- sich der jeweiligen kulturellen Sozialisation und Lebenszusammenhänge bewusst werden,

- Neugier, Offenheit und Verständnis für andere kulturelle Prägungen zu entwickeln,

- anderen kulturellen Lebensformen und -orientierungen zu begegnen, sich mit innen auseinanderzusetzen, dabei Ängste einzugestehen und Spannungen auszuhalten,

- das Anderssein des Anderen zu respektieren,

- den eigenen Standpunkt zu reflektieren, kritisch zu prüfen und Verständnis für andere Standpunkte zu entwickeln,

- einen Konsens über gemeinsame Grundlagen für das Zusammenleben in einer Gesellschaft bzw. einem Staat zu finden (STANJEK 2002: 273).

Wenn auch davon ausgegangen werden kann, daß die grundsätzliche Lebenssituation älterer Migranten von denselben physischen, faktischen und wirtschaftlichen Faktoren beeinflusst wird wie diejenige gleichaltriger Bundesbürger, muss doch berücksichtigt werden, dass sie in ihren Heimatländern individuell nach dem entsprechendem Normen- und Wertsystem sozialisiert wurden und ihre ethnischen und kulturellen Hintergründe nicht zuletzt in der hiesigen Altenhilfe anpassen müssen (DRK o.D.: 16).

Beispielsweise ist für die türkische Begrifflichkeit das Wort „Freizeit“ nicht existent und entsprechend unpassend. So betreiben nur die wenigsten türkischen Senioren ein Hobby, insbesondere gilt dies für diejenigen, die einem ländlichen Milieu entstammen. Die bäuerliche Tradition kennt keine Trennung zwischen Arbeit und Freizeit: „,'Boş zaman“ heißt wörtlich übersetzt „leere Zeit“. Dem traditionellen islamisch Denken entsprechend wäre „leere Zeit“ eine Art Sünde. [...] Zeit, in der man nicht wirtschaftlich tätig ist, wird mit sozialen und religiösen Tätigkeiten verbracht [...] d.h. [...] als Mitglied in einer Gemeinschaft [...] und nicht etwa [...], in der man individuellen Beschäftigungen und Interessen nachgeht" (CALICOGLU ET AL 2001: 93). 
Allerdings darf sich hierbei nicht das Stereotyp einer vermeintlich homogenen religiösen, politischen und soziokulturellen Prägung der älteren Türken durchsetzen, welches durch die im Projekt der AWO-Mannheim gewonnenen Erkenntnisse gründlich widerlegt wurde (TRIPPMACHER 1996:1).

Vielmehr bewahren Migranten individuell „in der neuen Aufnahmegesellschaft einige traditionelle Orientierungsmuster und übernehmen zugleich kulturelle Verhaltensweisen, die innen im neuen Land passend erscheinen. Dieser Prozess ist stark abhängig von positiven und negativen Erfahrungen in den verschiedenen Migrationsphasen (PAVKOVIC 2001: 37).

Eigentlich erübrigen sollte sich die Forderung, dass derartige kulturelle Hintergründe in der Pflege älterer türkischer Migranten dringend Berücksichtigung finden müssen.

Somit wachsen mit zunehmender Einbindung älterer Migranten in die Altenhilfe zugleich auch die Anforderungen an das Pflegepersonal: „[...] interkulturelles Arbeiten verlangt ein Mehr an Reflexion, an professionellem Können und ein Mehr an Bereitschaft, die eigene Praxis immer wieder in Frage zu stellen und gegebenenfalls zu korrigieren." (HINZ-ROMMEL 1998: 38).

STANJEK fasst die Rolle der Mitarbeiter einer interkulturellen, „lebensweltorientierten Pflege“ wie folgt zusammen: „Die Pflegekraft ist Gast in der Lebenswelt des Patienten oder Bewohners. Sie gehört für eine bestimmte Zeit oder auf Dauer zu seiner Lebenswirklichkeit. Deshalb muß sie die Lebensgeschichte (Biographie) der Pflegebedürftigen kennen und im Pflegeprozeß berücksichtigen (Biographischer Ansatz). Und sie muß die Identität des Pflegebedürftigen respektieren. Dazu gehören die persönliche Identität (z.B. Geschlecht, Alter, Körper), die soziale Identität (z.B. Sprache, Einstellungen, Denkmuster).“ (STANJEK 2002: 272).

Dass unter Mitarbeitern aus der Altenhilfe die Sensibilisierung für die spezifische Problematik älterer Migranten durchaus zugenommen hat, zeigt sich an ihrer grundsätzlich großen Motivation, sich mit der Thematik auseinanderzusetzen (MüLLER-WILLE 2001: 29).

EBNER weist aus schweizerischer Perspektive darauf hin, dass in Diagnostik, Beurteilung therapeutischer Möglichkeiten sowie Prognose kulturelle Kriterien bei Migranten einen großen Einfluß haben, wie sich nicht zuletzt anhand von Veröffentlichungen über Verfälschungen von Diagnosen und Problemen in der 
Therapie bei Migranten zeigt: soziokulturelle Probleme treffen - neben sprachlichen Schwierigkeiten -Migranten in besonderem Ausmaß (EBNER 2001: 34).

Migrationsspezifische Gutachter (...) müssen in jedem Einzelfall eruieren, in welchem Maße kulturspezifische Erlebnisweisen und Bewältigungsstile die zu diagnostizierenden Krankheitsbilder prägen. Sie benötigen Methoden zur Erfassung von fremden Kulturmustern, was wiederum die Reflexion der eigenen psychodiagnostischen Klassifikationssysteme als kulturelle Phänomene voraussetzt. Außerdem müssen sie auf Verzerrungseffekte im diagnostischen Prozess achten, die sich neben sprachlichen Verständigungsproblemen vor allem aus der Verschiedenheit in der Begegnung von Gutachter und Begutachtetem [sic] ergeben können - kulturell und in Bezug auf ihre gesellschaftliche Rollen (PAVKovic 2001: 37).

Immer wieder wird darauf hingewiesen, dass Einstellung und Verhalten gegenüber Gesundheit oder Krankheit kulturspezifisch geprägt seien. So gibt es „kulturspezifische Belastungssituationen, die zu unterschiedlichen, wiederum kulturspezifischen Bewältigungsstrategien und Bewältigungsstilen, zu unterschiedlichen Interpretationen eines Krankheitsbildes und schließlich zu kulturspezifisch ausgeprägten therapeutischen Interventionen führen“ (MARSCHALCK 2000: 36).

Kommunikationshindernisse zwischen Migranten und Deutschen - seien es unzureichende Sprachkenntnisse oder kulturspezifischer Besonderheiten erschweren es Migranten, dem medizinischen und Pflegepersonal Krankheiten und Beschwerden adäquat zu vermitteln. Aus dieser Problematik resultiert eine hohe Zahl an Fehldiagnosen, wie HINZ-ROMMEL an einem Beispiel verdeutlicht: So könne die Aussage "Mein Herz tut mir weh" häufig zu einer Überweisung zum Internisten führen anstatt zu der vielleicht eigentlich erforderlichen Behandlung durch einen sozialpsychiatrischen Dienst. Bestätigt werden diese Aussagen auch durch eine (leider undatierte) Untersuchung des Ethnomedizinischen Zentrums in Hannover (EMZ), welche eine extrem hohe Zahl sogenannter „PingpongÜberweisungen“ dokumentierte (HINZ-ROMMEL 1998: 36).

Zudem fehlen etlichen türkischen Migranten Kenntnisse über verschiedene alterstypische Krankheitsbilder wie Demenz, Diabetes, Dialysepflicht, Inkontinenz oder die Situation nach einem Schlaganfall. Umfragen des Seniorenbüro 
International in Essen zufolge waren derartige Kenntnisse bestenfalls marginal vorhanden (YILMAZ 2001: 17).

Ausgehend von der Annahme, sprach- und kulturkompetente Ressourcen in der (Alten-) Pflege seien nur sporadisch gegeben, entsprechende Kompetenzen, soweit vorhanden, würden nicht abgerufen und seitens der Regeldienste würde angemessene migrationssensible Konzept- und Angebotsentwicklung verzögert, wenn nicht gar verhindert, hat der sozialpolitische Handlungsbedarf die AWOOstwestfalen-Lippe zu einem einjährigen Projekt (bis März 2001) „Interkulturelle Kompetenz in der (Alten-)Pflege - Cross Cultural Care“37 bewogen (FREWER/JÄGER 2001: 39ff).

Derartigen Mißverständnissen ausschließlich durch die Einstellung ausländischer Mitarbeiter für die jeweiligen Migrantengruppen zu begegnen, stellt jedoch noch keineswegs eine Garantie zur interkulturellen Öffnung einer Einrichtung dar: Auch wenn zwar Umdenkprozesse durch die Arbeit in einem multikulturellen Team gefördert und erleichtert werden können, besteht dennoch gleichzeitig die Gefahr einer ethnischen Separation innerhalb der Einrichtung. In diesem Fall werden ausländische Mitarbeiter in die Rolle des Anwalts „ihrer Landsleute" gezwungen, die sie womöglich gar nicht ausfüllen wollen und die überdies einer tatsächlichen interkulturellen Arbeit im Wege stehen (HINZ-ROMMEL 1998: 38).

Neben diesen ersten Gehversuchen der kultursensiblen Versorgung von deutscher Seite wurde das Problem inzwischen auch auf türkischer Seite erkannt. SEEBERGER erwähnt das Entstehen erster Angebote von konfessionell gebundener Altenhilfe und -betreuung, die von Moscheen, -vereinen oder anderen türkischislamischen Organisationen aufgebaut werden. Die Finanzierung basiert auf einem Selbsthilfeansatz über finanzielle Eigenbeteiligung aus der türkischen Volksgruppe heraus (SEEBERGER 2001: 4.).

37 Das Projekt war angegliedert an das transnationale LEONARDO DA VINCI-Programm der Europäischen Union, das auf berufliche Aus- und Weiterbildung als zentrales Instrument gesellschaftlicher Innovation abzielt. Wie auch beispielsweise in Dänemark oder Italien strebt das Programm derzeit vor allem nach Ausbildung von MigrantInnen und Flüchtlingen für Pflegeberufe, um so Multiplikatoren für Sozial- und Pflegebereiche innerhalb der Migrantengruppen zu gewinnen (FREWER/JÄGER 2001, S. 39) 
Bedenkenswert ist in diesem Zusammenhang die Schilderung HinzRommels über die Anstellung eines Psychologen aus der Türkei in der psychosozialen Beratungsstelle in Nürnberg, nachdem dort ein starker Bedarf und Notwendigkeit muttersprachlicher Betreuung anerkannt worden war. Trotz eigentlich entgegengesetzter Konzeption fand in der Beratungsstelle eine Trennung der Klientel nach den jeweiligen Nationalitäten statt, bis sich deutsche Kollegen über die vielen Migranten in Wartezimmern und Fluren beschwerten und damit eine überfällige Diskussion zur interkulturellen Konzeption der Einrichtung anstießen (HINZ-ROMMEL 1998: 97f).

Der Bedarf von kultursensibler Versorgung ist gegeben: „Insgesamt machten wir die Erfahrung, daß Information und Aufklärung zu Fragen der sozialen Sicherung die Bevölkerungsschicht älterer Migrantelnnen anscheinend kaum erreicht. Es wäre dingend notwendig, das Angebot an muttersprachlichen Beratungen und adäquatem Informationsmaterial flächendeckend auszubauen." (Calicoglu et al 2001: 102f).

\subsection{Begriffsentwicklung: Alte Migranten}

In dem Kontext der vorliegenden Arbeit ist wie bereits zuvor angedeutet von sogenannten 'alten Migranten' die Rede. Den Hintergrund stellt der Faktor, dass Alter nicht immer nur rein biologisch messbar ist. In diesem Kontext ist somit ein Mensch im Fokus, der psychisch und biologisch in der Fremde lebt und dem ein Status der Zuwanderungsgeschichte zugeschrieben wird. Dabei muss der Migrant nicht unbedingt bereits ins Rentenalter von 65 Jahren eingetreten sein um als 'alt' zu gelten. Auch 55- Jährige können bereits gemeint sein, da diese auf verschiedene (z.B. stressbedingt auf psycho-physischer) Ebene 'vorgealtert' sind. Ein Grund kann eine Lebensbelastung sein, die ihre Grundlage in der sozioökonomischen Herkunft findet. Ein weiterer Grund kann die biographische Entwicklung in der 'Fremde' sein. Der Begriff des alten Migranten dient somit der Abgrenzung gegenüber der westlichen Alterszuschreibung von 65+, da sich die gealterten Migranten selbst bereits deutlich früher als 'alt' bezeichnen. Ein alter Migrant Anfang 50 befindet sich somit in einer rechtlichen Grauzone, da er noch 
keinen Anspruch auf die Versorgungsbereiche für die 'eigentlich' Alten hat. Da das familiäre Versorgungssystem aber nach und nach an Stabilität verliert und das Großfamilienklima ebenso nicht mehr auf Dauer intakt bleibt (vgl. Ambivalenzen der kulturellen Identität in C.3 $\rightarrow$ Ausblick), sieht sich der alte Migrant als Folge oder Teil des Akkulturationsstresses mit existenziellen Problemen konfrontiert. Wird diesem Menschen nun noch mit Ausweisung aus dem Land gedroht, falls er keinen Integrationskurs besucht und erfolgreich abschließt, erhöht sich die Belastung erneut. Kann man unter solcher Belastung noch eine fremde Sprache oder Kultur wirklich (kennen-)lernen?

\section{Lernen}

Die abschließende Frage aus dem vorherigen Kapitel, soll die Basis für das folgende Kapitel darstellen. Was ist Lernen, Wissen oder Bildung überhaupt? Und wie kann man 'gut' lernen - vor allem als Migrant, der einem "Akkulturationsstress' ausgeliefert ist?

\subsection{Definition(en) - Bildung = Wissen $=$ Lernen $?$}

Die Begriffe Bildung, Wissen und Lernen stehen auf den ersten Blick untrennbar nebeinander. Gemeinsprachlich inflationär auch gelegentlich synonym gebraucht besagen sie jedoch Unterschiedliches.

FLECHSIG UND HALLER (2003) verzichten begründet in diesem Zusammenhang auf eine Ein-Satz-Definition des Begriffs „Didaktik“, weil sie aufgrund gesellschaftlicher Unterschiede den individuellen Bedingungen des Lernens Sorge tragen möchten.

„Dass "Didaktik" etwas mit Unterricht und Lernen zu tun hat, weiß wohl jedermann. Dass der Begriff eine Geschichte hat und dass bis in die Gegenwart hinein die unterschiedlichsten Auslegungen und Definitionen vorgenommen worden sind, sei dem Leser versichert.“(LANDWEHR 2007: 8) 
Die Autoren leiten hin zum „organisierten Lernen“ und zum „didaktischen Handeln“ (ebd.: 9), wobei „[...] Zur besseren Verständigung bedarf jedoch auch der Begriff "Handeln"' einer ersten Präzisierung. Zunächst vermittelt das Wort "Handeln" die Auffassung handgreiflichen Einwirkens auf die Umwelt, auf Menschen und Sachen. Doch kann die Einwirkung auch mittels Sprache geschehen, keineswegs nur der Befehlssprache, sondern ebenfalls durch beschreibende Aussagen. Schließlich kann Handeln auch durch andere Formen symbolischen oder nichtsymbolischen Ausdrucks erfolgen: Gesten, Musik, Bilder oder Werkzeugherstellung. All dies trifft auch auf didaktisches Handeln zu: mittelbares oder unmittelbares Einwirken auf Menschen und Sachen." (ebd.: 9).

Didaktisches Handeln findet auf verschiedenen Ebenen (Felder oder Räume) statt:

- Gestaltung der institutionellen, ökonomischen, personellen und konzeptionellen Rahmenbedingungen (A-Ebene)

- Gestaltung übergreifender Lehrplan- und Schulkonzepte (B-Ebene)

- Gestaltung von Lernbereichen und Unterrichtskonzepten (C-Ebene)

- Gestaltung von Unterrichtseinheiten (D-Ebene)

- Gestaltung von Lehr-/Lernsituationen (E-Ebene)

(ebd.: 9f)

Diese Ebenen des didaktischen Handelns greifen in das von DöRPINGHAUS, POENITSCH und WIGGER (2008) beschriebene Verständnis von Bildung, welche als „Beziehungen und Verhältnisse zur Sprache bringt, die - erstens - Menschen zu sich selbst, - zweitens - zu ihren Mitmenschen und - drittens - zum Gesamt der Welt eingehen bzw. eingegangen sind“(LANDWEHR 2007: 10).

Diese Verschränkungen der Definitionen von Didaktik und Bildung führen unweigerlich zur Auseinandersetzung mit der Begrifflichkeit der Persönlichkeit im kulturellen Kontext, das auch in der Schwierigkeit der Definition des Wissens seinen Ausdruck findet. Im Grunde kann es kein Wissen geben, „sondern wird von Gesellschaften immer nur zur Bewältigung ihrer jeweiligen Realitäten hergestellt und angewandt." Die Gültigkeit von Wissen ist begrenzt durch den gesellschaftlichen Rahmen und einen „bestimmten historischen Zeitraum“, in dem Wissen einen „Wissenstatus reklamieren kann“ (ebd.: 802).

Nach LANDWEHR habe das Wissen als ein Produkt der Menschen seinen 
Ursprung in soziokulturellen Realitäten. Das Verhalten in solchen Realitäten sei das Resultat des Wissens, welche „einerseits innerhalb bestimmter kultureller Kontexte operieren und Normen, Werte und Kategorien transportieren, andererseits aber auch solche Formen der Bedeutung hervorbringen.“ (ebd.: 803)

Nachvollziehbar sollte nach den Darstellungen der vorherigen Kapitel nun sein, dass Kultur Identität stiftet. Da Wissen und Bildung elementare Bausteine einer jedweden Kultur sind, sind sie somit ebenso kulturstiftend, wie AUERNHEIMER 2007 darlegt:

Das für die multikulturelle Gesellschaft und damit für die interkulturelle Pädagogik leitende Prinzip der Anerkennung verweist auf den Begriff der Identität und auf den Zusammenhang von Identität und Kultur. Die Identitätsproblematik lässt sich etwas vereinfacht, in die Fragen „Wer bin ich? Wer möchte ich sein?" übersetzen, ohne dass diese Fragen immer ausdrücklich gestellt werden müssen. Das Ideal der Selbstreflexion ist etwas, was sich mit der Vorstellung von Bildung verbindet, wobei die Selbsterkenntnis nach Ansicht der Klassiker nur möglich ist über Arbeit und Kommunikation.

Das verlangt die tätige Aneignung der vergegenständlichten Welt, also der jeweiligen Kultur. Die Erörterung des Bildungsbegriffs wird nicht nur durch die Nähe zum Identitätskonzept nahe gelegt, sondern auch deshalb, weil die allgemeine Bildungstheorie mehrere Ausschlussmöglichkeiten für die Begründung interkultureller Bildung bietet. Andererseits wird auch an nationalistische Deformationen in der Begriffsgeschichte zu erinnern sein.

Die Bildungsidee und das Identitätsproblem haben eine historische Problemkonstellation als gemeinsamen Ausgangspunkt, die den Anfang der "Moderne“ oder in anderen Worten der "bürgerlichen Gesellschaft“ markiert. Es ist der Beginn des Prozesses der Individualisierung, der sich unterschiedlich zurückdatieren lässt, bis zur Renaissance oder bis zum Zeitalter der Aufklärung. Die Frage der Selbstdefinition, des eigenen Lebensentwurfs und des Ortes im gesellschaftlichen Ganzen stellt sich nämlich mit der beginnenden Auflösung der traditionellen ständischen Ordnung und der Erfindung des abstrakten Menschen (vgl. TAYLOR 1993), von dem Moment an, wo der/die Einzelne der Gesellschaft unmittelbar gegenüber gestellt ist, weil nicht mehr soziale Gruppen den Zusammenhang vermitteln, sondern der Markt, der Staat - allgemein: die "Systeme“. (AUERNHEIMER 2007: 64f)

Führt man obigen Begriff von Bildung weiter aus und zieht erste Verbindungen zur Neurodidaktik und Neurobiologie der Stressverminderung worauf im 4. Kapitel näher eingegangen wird, kommt man nicht umhin, nochmals AUERNHEIMER zu zitieren, der eben diese Aspekte anspricht:

Im pädagogischen Konzept von WolfGang NieKE (2000, 1. Aufl. 1995) finden kulturelle oder "ethnische“ Differenzen (dazu S. 38) eine große Beachtung. Seine Hauptintentionen kann man vermutlich als „aufgeklärten Ethnozentrismus“ und Befähigung zum Dialog (dazu Kap. 6.5) kennzeichnen. Nieke geht von „der Unvermeidlichkeit des Eingebundenseins in die Denk- und Wertgrundlagen der eigenen Lebenswelt" (S. 205) aus und hält ein Bewusstsein davon für das erste Ziel interkultureller Bindung. Jenes starke Eingebundensein macht auch die Reaktion der Befremdung, zum Teil der Angst bei der Konfrontation mit anderen Lebenswelten verständlich, weshalb das „Umgehen mit Befremdung" für Nieke das zweite Ziel ist. Die Bearbeitung der Gefühle soll zum Beispiel die Verwandlung von Angst in Neugier ermöglichen [Hervorhebung I.Ö.].

$[\ldots]$ 
Den gefährlichen Implikationen, die der eigenen Intention widersprechen würden, versucht Nieke zu begegnen, indem er die Betonung von Gemeinsamkeiten, die „Ermunterung zur Solidarität" und die Entwicklung einer globalen "Wir-Identität" propagiert, wobei allerdings mit eigenen Einwänden nicht spart [Hervorhebung I.Ö.]. Es sei auch schon vorweggenommen, dass für Nieke der Umgang mit kulturell bedingten normativen Differenzen zentral ist. Zunächst ist hier aber nur die Bearbeitung von Differenzerfahrungen Thema [Hervorhebung I.Ö.]. [...] (AUERNHEIMER 2007: S. 133f)

\subsection{Theorien}

\section{Überblick über die Entwicklung der Lernpsychologie}

In der Entwicklung der modernen Lernpsychologie entstand eine Vielzahl von psychologischen Schulen und Lerntheorien, von denen einige besondere Bedeutung erlangt haben. Im Folgenden soll ein Abriss der wichtigsten lernpsychologischen Entwicklungen gegeben werden, wobei auf die bedeutendsten Vertreter der einzelnen Schulen sowie auf Besonderheiten ihrer Theorien eingegangen wird.

\section{Edward L. Thorndike: Verbindungslehre}

Die Verbindungslehre des amerikanischen Psychologen und Pädagogen EDWARD THORNDIKE (1874-1949) benannte als Grundlage des Lernens die Verknüpfung zwischen Sinneseindrücken und Handlungsimpulsen. Mit dieser Vorstellung begründete er die sogenannte Reiz-Reaktions-Psychologie des Lernens.

"Versuch und Irrtum" oder genauer "Lernen durch Auswahl und Verbindung“ stellen die wichtigsten Konzepte seiner Theorie dar, wobei der Lernende in einer Problemsituation durch das Ausprobieren verschiedener Verhaltensweisen zum Ziel zu kommen versucht. Erfolgreiche Verhaltensweisen werden dabei gespeichert, also gelernt - während nicht erfolgreiche Verhaltensweisen als nutzlos abgespeichert und verworfen werden. Erfolgreiche Reaktionen werden auch in der Ursprungssituation ähnlichen Situationen angewendet (Generalisierung).

Bekannt geworden ist in diesem Zusammenhang THORNDIKES Problemkäfig - eine Konstruktion für Tierexperimente, mithilfe derer die beschriebenen Thesen überprüft und bestätigt werden konnten. Thorndike war aufgrund des langsamen Lernverlaufs der Ansicht, dass Versuchstiere nicht durch Überlegungen zum Ziel 
gekommen waren, sondern allein durch die allmähliche Auswahl richtiger und die Verwerfung falscher Handlungsweisen lernen. Er formulierte das mechanistische Gesetz der Auswirkung, das das Auftreten oder Nicht-Auftreten einer zeitnahen Belohnung entscheidend für die erneute Verwendung oder Verwerfung von Reizreaktionen macht. Damit erweiterte er die bereits bestehenden Gesetze der Bereitschaft (zum Lernen ist ein Bedürfnis notwendig, das befriedigt werden soll) und der Übung (Verhaltensweisen müssen wiederholt werden, um sie dauerhaft zu lernen). ${ }^{38}$

Lernen war somit nach Thorndike zum größten Teil durch direkte Verknüpfungen von Verhaltensreaktionen mit Situationen und die automatische Wirkung des Gesetzes der Auswirkung erklärbar, wobei sich das Lernen bei Tieren und Menschen nicht grundsätzlich unterschied.

\section{Iwan P. Pawlow: Klassische Konditionierung}

Der russische Physiologe IWAN PAWLOW (1849-1936) war der Begründer der klassischen Konditionierung. Berühmt geworden ist PAWLOWS Experiment zum Speichelfluss bei Hunden, wobei er durch wiederholte gleichzeitige Darbietung einen sogenannten unkonditionierten Reiz (Hundefutter) mit einem konditionierten Reiz (Aufleuchten einer Lampe) verband. Die ursprünglich unkonditionierte, automatische Reaktion (Speichelfluss des Hundes bei der Darbietung von Futter) ließ sich nach ausreichend vielen Durchgängen nun auch allein durch das Leuchten der Lampe hervorrufen und wurde so zur konditionierten Reaktion. Entscheidend für die Bildung einer konditionierten Verknüpfung ist die zeitliche Nähe der beiden zu konditionierenden Reize.

Bei alleiniger Präsentation des konditionierten Reizes nimmt die konditionierte Reaktion allmählich wieder ab und verschwindet schließlich ganz (experimentelle Extinktion). Die sogenannte Spontanerholung bezeichnet das Wiederauftreten der konditionierten Reaktion nach einiger Zeit ohne jegliche Wiederholungen. ${ }^{39}$

\footnotetext{
38 Auf weitere von THORNDIKE verfasste Prinzipien und Gesetze oder Modifikationen der oben genannten kann hier nicht eingegangen werden. Sie sind z.B. in BOWER \& HILGARD (1983), S. $48 f f$ nachzulesen.

39 Weiterführend: Bower \& Hilgard (1983), Kapitel 3
} 


\section{Edwin R. Guthrie: Konditionierung durch Kontiguität}

Die Lerntheorien des amerikanischen Psychologen EDWIN GUTHRIE (18861959) waren gewissermaßen anders gewichtete Weiterentwicklungen und veränderte Interpretationen der Lehren von THORNDIKE und PAWLOW. GUTHRIE war ein früher Behaviorist. Der Behaviorismus fasst Verhalten als Ergebnis einer Wechselwirkung von Reiz und Reaktion auf. Behavioristen akzeptieren nur offen beobachtbares Verhalten als Grundlage der psychologischen Forschung, um den Anspruch auf Objektivität erheben zu können. GUTHRIES einziges Lerngesetz der Assoziation durch Kontiguität (= Nähe in Zeit oder Raum) beschränkt sich also auf die beobachtbare Verhaltensebene:

„Eine Kombination von Reizen, die mit einer Bewegung einhergeht, pflegt beim erneuten Auftreten diese Bewegung nach sich zu ziehen." (GUTHRIE, 1935)

In Kontrast zu THORNDIKES Gesetz der Übung ist GUTHRIE der Ansicht, dass ein Reizmuster bereits bei seinem ersten gemeinsamen Auftreten mit einer Reaktion seine volle Verknüpfungsstärke erreicht. Das Lernen findet demnach durch eine einzige Darbietung statt. Erwiesene Leistungsverbesserungen durch Übung erklärte GUTHRIE damit, dass komplexe Handlungen aus vielen einzelnen Bewegungen bestehen, von denen jede erst einmal gelernt und an verschiedene Situationen angepasst werden muss. Erst wenn alle Reizmuster zum erwünschten Verhalten führen, wird eine Fertigkeit beherrscht. ${ }^{40}$

\section{Clark L. Hull: Systematische Verhaltenstheorie}

Der amerikanische Psychologe CLARK HULL (1884-1952) entwickelte mithilfe von THORNDIKES Gesetz der Auswirkung eine behavioristisch-mechanistische Lerntheorie, bei der er jeden Bezug auf ein Bewusstsein des Organismus vermied. Er versuchte, zielgerichtetes Verhalten und das Phänomen der Einsicht zu erklären, indem er nicht nur die situativ stimulierende Umgebung des Individuums, sondern weitere Einflüsse benannte und in seine Betrachtungen einbezog.

HULL war der Ansicht, dass sich Einflüsse wie die lebensgeschichtliche Entwicklung, der Gesundheitszustand oder die biologischen Bedürfnisse eines

40 Genauer: BOWER \& HILGARD (1983: Kapitel 4) 
Organismus in einigen intervenierenden Variablen zusammenfassen ließen. Diese Variablen stünden als Vermittler zwischen Ursache und beobachtbarem Verhalten -so würde beispielsweise die Ursache „Salzige Nahrung“ mit der intervenierenden Variable "Durst" das Verhalten „Anstrengung für Wasser“ hervorrufen. HULL postulierte seine Theorie in mathematischer Form, wobei er unter Einbeziehung von positiven Verstärkern und negativen beeinflussenden Faktoren die Geschwindigkeit und die Stärke der resultierenden Reaktion berechnete.

HuLLS Lerntheorie wurde vielfach weiterentwickelt. ${ }^{41}$ NEAL E. MILLER (1909200, ein Schüler HuLLS, wandte seine Konzepte auf verschiedene Verhaltensphänomene an und untersuchte als Pionier des sogenannten Biofeedbacks u.a. die physiologischen und biochemischen Substrate von Motivation und Belohnung. ORVAL H. MoWreR (1907-1982) entwickelte die sogenannte Zwei-Faktoren-Lerntheorie, die Reaktionen des Zentralnervensystems von Reaktionen des autonomen Nervensystems unterschied und durch verschiedene Lernvorgänge in den beiden Systemen erklärte.

\section{Forschung zum Lernen bei Menschen}

Parallel zu der beschriebenen theoretischen Konditionierungsforschung bei Tieren entwickelte sich ein Forschungszweig über das assoziative Lernen bei erwachsenen Menschen, wobei an dieser Stelle ein besonderer Fokus auf das verbale Lernen gelegt werden soll.

Der Begründer der experimentellen Erforschung des Gedächtnisses war der deutsche Psychologe HeRRMAnn Ebbinghaus (1850-1909). Indem er eichbare Erinnerungseinheiten in Form sinnloser Silbenlisten einsetzte, erforschte er die Auswirkungen der Listenlänge, der Lerndauer, der Anzahl der Durchgänge und die Länge der Behaltensleistung. Er entwickelte die Lernkurve, die den Erfolgsgrad des Lernens über die Zeit beschreibt, und die Vergessenskurve, die den Grad des Vergessens beschreibt. Weiterhin beschäftigte EBBINGHAUs sich mit Assoziationsund Paarassoziationslernen, Interferenzeffekten und Transferleistungen. ${ }^{42}$

\footnotetext{
41 Weiterführende Informationen in BOWER \& HILGARD (1983: 162ff)

42 BOWER \& HILGARD (1983:Kapitel 6)
} 


\section{Burrhus F. Skinner: Operante Konditionierung}

Der amerikanische Psychologe und Verhaltensforscher B. F. SKINNER (19041990) untersuchte Verhaltensweisen, die durch die der Handlung folgenden Konsequenzen beeinflusst wurden. Für diese Experimente benutzte er die Skinner-Box, in der Versuchstiere für einen Hebeldruck mit Futter belohnt wurden. Im Gegensatz zur klassischen Konditionierung wird bei der operanten Konditionierung ein spontan gezeigtes Verhalten durch die positiven oder negativen Auswirkungen des Verhaltens entweder verstärkt oder verringert. Beispielsweise wird die Wahrscheinlichkeit vergrößert, dass ein Junge erneut pünktlich nach Hause kommt, wenn inn seine Mutter bei diesem Verhalten mit einem Geschenk belohnt hat. Dagegen wird ein Hund wahrscheinlich nicht mehr an einem Menschen hochspringen, wenn er dafür bereits bestraft worden ist. Der Ablauf der klassischen Konditionierung (auf einen Reiz folgt eine Reaktion) wird hier also erweitert: Auf ein Verhalten folgt eine Konsequenz, die wiederum das erneute Auftreten des Verhaltens beeinflusst.

\section{William K. Estes: Die Stimulus-Auswahl-Theorie}

Der amerikanische Wissenschaftler WILLIAM ESTES (geb. 1919) war der Ansicht, dass Individuen dadurch lernen, dass sie Situationen, in denen sie sich unangemessen verhalten haben, neue und passendere Verhaltensweisen zuordnen. Organismen lernen also innere Repräsentationen für äußere Ereignisse und bilden Assoziationen zwischen diesen.

ESTES' Stimulus-Auswahl-Theorie (entwickelt um 1950) behandelt Lernen und Leistung als probabilistische Prozesse, analysiert sie also hinsichtlich ihrer Wahrscheinlichkeit. Dies ist begründet durch die vielen unbekannten oder nicht messbaren Ursachen des Verhaltens, die eine definitive Vorhersage meist unmöglich machen. Die bestmögliche Vorhersage ist also mit einer gewissen Wahrscheinlichkeit behaftet. Während des Lernvorganges sollte die Wahrscheinlichkeit einer richtigen Reaktion immer weiter ansteigen, bis sie schließlich $100 \%$ beträgt. 


\section{Moderne Entwicklungen}

Durch die Errungenschaften der modernen Technik und die Entwicklung der bildgebenden Verfahren in der Hirnforschung konnten in den letzten Jahrzehnten bedeutende Fortschritte beim Verstehen von Lernvorgängen gemacht werden.

In den späten sechziger Jahren des 20. Jahrhunderts versuchte eine Arbeitsgruppe um NEAL MILLER erstmals, einige viszerale Reaktionen operant zu konditionieren, indem sie eine Ratte durch Stimulation des Lustzentrums im Gehirn belohnten, wenn ihre Herzfrequenz nach Darbietung eines Tons anstieg. Diese Experimente zeigten, dass Tiere in der Lage sind, Veränderungen ihrer Herzgefäß- und Organreaktionen zu lernen, wenn dies zu Verstärkung führt.

Diese Ergebnisse gaben Anlass zur Forschung im Bereich des Biofeedbacks. Dabei werden ursprünglich unbewusste körperliche Vorgänge eines Organismus (z.B. der Blutdruck) für ihn bewusst gemacht, so dass er lernen kann, Einfluss darauf zu nehmen. In den 70er Jahren des 20. Jahrhunderts konnten Miller und Brucker querschnittsgelähmten Probanden beibringen, ihren Blutdruck zu erhöhen, so dass sie aufrecht stehen konnten, ohne ohnmächtig zu werden (BOWER UND HILGARD 1983: 373)

\section{Beziehung und Lernen}

Auch unter neurobiologischen Aspekten spielt die Beziehungsebene für das Lernen eine entscheidende Rolle, wie z.B. RAUFELDER und MOHR an aktuellen Untersuchungen mit Schülern zeigen:

Zwei psychologische Konzepte können hier vermittelnd wirken: erstens das der Selbstwirksamkeitserwartung, also die subjektive Überzeugung, neue oder schwierige Anforderungen aus eigener Kraft bewältigen zu können. Ist sie stark ausgeprägt, fördert das die Fähigkeit zur Selbstregulation, die auch Schüler brauchen, um manchen Verlockungen von außen zu widerstehen [...]

Zweitens spielt auch die Art der Lernmotivation eine Rolle. Der Anreiz, gute Noten nach Hause zu bringen, kann vom Wunsch nach Anerkennung getrieben sein (»extrinsisch«). Intrinsische Motivation speist sich dagegen eher aus dem Interesse und der Freude am Lerngegenstand. Gemäß der von den Psycholgen Richard Ryan und Edward Deci von der University of Rochester (US-Bundesstaat New York) in den 1980er Jahren entwickelten Theorie der Selbstbestimmung (self-determination theory) kann extrinsische Motivation letztlich auch die intrinsische fördern: So mag Bestätigung durch das soziale Umfeld das eigene Kompetenzerleben stärken - der Betreffende traut sich selbst mehr zu, und das hebt wiederum die Laune beim Lernen. [...] (RAUFELDER UND MOHR 2010: 74ff) 


\subsubsection{Lebenslanges Lernen und Lernen im Alter}

\section{Einleitung und Begriffserklärung}

In den Schlussfolgerungen des Europäischen Rats von Lissabon im März 2000 wurde festgestellt, dass sich Europa unbestreitbar auf dem Weg in das Zeitalter des Wissens befindet - mit vielen Konsequenzen für das kulturelle, wirtschaftliche und soziale Leben. Sich rasch wandelnde Lern-, Lebens- und Arbeitsmuster würden erfordern, dass sich die Menschen an den Wandel anpassen und sich traditionelle Handlungsmuster teilweise ändern müssen. Der erfolgreiche Übergang zur wissensbasierten Wirtschaft und Gesellschaft müsse mit einer Orientierung zum lebenslangen Lernen einhergehen. Europas Bildungsund Berufsbildungssysteme stehen im Mittelpunkt der bevorstehenden Veränderungen (EUROPÄISCHER RAT, 2000).

Der Begriff des lebenslangen Lernens ist nicht einheitlich definiert und bietet großen Interpretationsspielraum. Eine Arbeitsdefinition der Europäischen Kommission beschreibt lebenslanges Lernen als ,jede zielgerichtete Lerntätigkeit, die einer kontinuierlichen Verbesserung von Kenntnissen, Fähigkeiten und Kompetenzen dient." (KommissiOn DER EUROPÄISCHEN GEMEINSCHAFTEN, 2000).

Im Glossar der UNESCO findet sich folgende Definition: (Lifelong Learning is) „the concept of learning as a process that continues throughout life to address an individual's learning needs. The term is used widely in adult education to refer to learning processes in many forms and at many levels." (UNESCO, 2005).

Sinnvoll ist hier eine Unterscheidung der beiden Begriffe „Education“ und „Learning“, da diese englischen Begriffe auch im Deutschen häufig verwendet werden. GERLACH (2000) äußert sich dazu folgendermaßen:

\footnotetext{
Die aus dem englischen Sprachraum übernommene Terminologie „lifelong learning/ lifelong education" wird in den meisten Fällen synonym verwendet, wohingegen der Begriff „learning" teilweise auch präziser als eigentlicher Prozess der Veränderung durch Erfahrung definiert wird und „education“ einen von außen gesteuerten Lernprozess beschreibt [...]. (Es verdeutlicht sich) eine prinzipielle Verwendung des Terms "education“ im Zusammenhang mit institutionalisierter, schulischer Bildungsform, die von außen gesteuert oder entworfen wird und „learning“ für selbstständigere, unabhängige, direkte Lernerfahrungen verwendet. (GERLACH, 2000).
}

Gerlach extrahiert aus verschiedenen historischen Ansätzen und 
Betrachtungen zwei grundsätzliche Kontexte, die lebenslanges Lernen erforderlich machen:

\begin{abstract}
Einerseits steht lebenslanges Lernen in einer grundsätzlichen Bedeutung biologisch und evolutionistisch bedingter Notwendigkeit. In diesem Verständnis existiert es seit der Entstehung des Menschen und leitet sich aus seiner 'Unfertigkeit' ab. Die Aneignung von Fertigkeiten und Kenntnissen sowie die Entwicklung neuer Kompetenzen orientiert sich an den Anforderungen und Situationen der unmittelbaren Umwelt und sichert somit ein Überleben [...]. In diesem Sinne ist Erziehung und Bildung [...] ein lebenslang notwendiger Prozess. Andererseits kennzeichnet sich die Entwicklung von lebenslangem Lernen durch eine enge Verknüpfung mit wirtschaftlichen und technologischen Fortschritten der Industrieländer. In diesem Zusammenhang entstehen unterschiedliche Bereiche von Lernbedürfnissen, die sich aus qualifikatorischen Anforderungen der Arbeit bedingen und zunächst eine Aneignung von Fertigkeiten und Kenntnissen für berufliche Zusammenhänge beinhalten sowie die Gestaltung und Bewältigung von außerberuflichen Situationen einschließen. (GERLACH 2000: 157)
\end{abstract}

Die Frage nach Ursprung und Definition des Begriffs „Lebenslanges Lernen" ist nicht eindeutig zu beantworten. Aufgrund der allgemeinen Formulierung des Terms und der vielen gebrauchten Synonyme können nur Tendenzen und wesentliche historische Entwicklungen beschrieben werden, die diesen Bereich des Lernens betreffen.

\title{
Geschichtliche Eckpunkte der Entwicklung des Konzepts „Lebenslanges Lernen"
}

\section{Griechenland, Antike}

Die Geschichte des Lebenslangen Lernens beginnt bereits in der Antike (um 1100 v. Chr. bis um 500 n. Chr. (ENCARTA, 2009). Damals definierte der griechische Philosoph PLATON in seiner Schrift „Politeia“ (PLATO, 2000) die Philosophie als einen Erwerb von Wissen. Durch die Dialektik und das fortlaufende Denken könne die Wahrheit und das Sein erkannt werden. Das Lebenslange Lernen ist deshalb bedeutsam für PLATON, weil die Philosophen in diesem Sinne Suchende, also fortwährend Lernende sind (textlog.de, 2004).

\section{Frankreich, 1792}

Im Jahre 1792 legte CONCORCET der französischen Nationalversammlung einen „Bericht und Entwurf einer Verordnung über die allgemeine Organisation des 
öffentlichen Unterrichtswesens“ vor. In dieser Schrift betont er die Notwendigkeit, das Lernen auch nach der Schullaufbahn nicht aufzugeben:

Wir haben schließlich darauf geachtet, daß der Unterricht die Individuen nicht in dem Augenblick preisgeben darf, in dem sie die Schulen verlassen; daß er vielmehr alle Altersgrenzen umfassen muss, daß es keine gibt, in der zu lernen nicht nützlich und möglich ist; und daß dieser Sekundarunterricht um so notwendiger ist, je engere Grenzen dem Unterricht in der Kindheit gezogen waren. (LENGRAND 1972: 16).

\section{Amerika, ab 1874}

Auch in den USA zeigte sich schon zu Gründungszeiten ein Bildungsbewusstsein, das durch Freiheits- und Demokratiestreben sowie den Pioniergeist des neuen Staates geprägt war. Dies zeigte sich besonders in einer Bewegung der Erwachsenenbildung, der sogenannten Chautauqua-Bewegung, in den ländlichen Gebieten der USA vom Ende des 19. Jahrhunderts bis zum Beginn des 20. Jahrhunderts (CANNING, 2005). Die Bewegung wurde durch die Gründung des Chautauqua-Institutes im Jahr 1874 durch J. H. VINCENT und L. MILLER initiiert. $\mathrm{Zu}$ dieser Zeit waren die Folgen des amerikanischen Bürgerkriegs noch spürbar, viele Menschen zogen wegen sagenhafter Goldvorkommen nach Westen und nur $3 \%$ aller amerikanischen Kinder erreichten die High School.

Chautauqua war anfangs als Sommerschule für methodistische Sonntagsschullehrer konzipiert, die Themen und Lehrgebiete wurden jedoch rasch erweitert. Zusätzlich zur Bildung existierte in Chautauqua ein gut durchdachtes Freizeitprogramm, das der Erholung dienen sollte. Als Form der Lehre wurden unterhaltende, wie bildende Elemente miteinander kombiniert. Das Institut bot der Gemeinschaft die Möglichkeit, im Zuge von drei- bis siebentägigen Veranstaltungen gemeinsam Vorlesungen zu verschiedenen Themen zu hören, Theaterstücken, Konzerten und ersten Kinovorführungen beizuwohnen, und vor allem über diverse politische, soziale und kulturelle Themen nachzudenken und zu diskutieren. Das Ziel von Chautauqua war es, Bildung und Kultur zu verbreiten, um ein mündiges Bürgertum zu schaffen. Präsident Theodore Roosevelt nannte die Veranstaltungen „the most American thing in America“.

1878 entwickelte VINCENT den „Chautauqua Literacy and Scientific Circle“, an dem über 8400 Personen teilnahmen. Innerhalb von 10 Jahren stiegen die Zahlen auf 100.000 Teilnehmer. 1883 wurde die Chautauqua Universität gegründet, die als erste Einrichtung in den USA auch Fernstudiendiplome 
ausstellte.

Ausgehend von der ersten Chautauqua im Bundesstaat New York wurden überall in den USA unabhängige Chautauquas gegründet, die das Konzept verbreiteten. 1890 erreichte die Zahl der „Unabhängigen“, wie sie auch genannt wurden, bereits 200. Ihre goldene Zeit erlebte die Chautauqua-Bewegung zwischen 1905 und 1928. Am Höhepunkt ihrer Entwicklung waren ca. 1000 permanente und mehr als 10.000 wandernde Chautauquas entstanden. Zum größten Chautauqua-Zentrum in Boulder im Bundesstaat Colorado pilgerten in jeder Saison ca. 100.000 Menschen. Bis 1932 verschwanden fast alle WanderChautauquas wieder. Rundfunk, Film und Fernsehen sowie die zunehmende Mobilität trugen erheblich dazu bei.

Im Jahr 1999 veranstaltete man in Boulder zum 100. Geburtstag des dort einst ansässigen Chautauqua den „1. Internationalen Dialog“, der die Tradition wieder aufleben lassen sollte. Dort wurden verschiedene Themen, unter anderem das Bildungswesen, vorgestellt und diskutiert.

\section{Brasilien: Paulo Freire}

Der einflussreiche brasilianische Pädagoge PAULO FREIRE (1921-1997) setzte nach dem 2. Weltkrieg ein Alphabetisierungsprogramm in den Slums und Arbeitersiedlungen Brasiliens durch. Zu diesem Zeitpunkt waren Analphabeten in Brasilien nicht wahlberechtigt, so dass die Alphabetisierung eine Kampagne von hoher politischer Relevanz darstellte. FREIRE sah den Prozess der Alphabetisierung und der Bildung als unabdingbares Mittel zur Selbstbefreiung der unterdrückten Bevölkerung von der unterdrückenden Elite. „First, I would like to reaffirm that I always saw teaching adults to read and write as a political act, an act of knowledge, and therefore as a creative act." (FREIRE, 1983)

Nach einer durch Hunger und Armut geprägten Kindheit schloss FREIRE ein Jurastudium ab und heiratete eine Grundschullehrerin, wodurch er in Kontakt mit Fragen der Erziehung kam. Im Jahr 1946 führte er für den Sozialdienst des Arbeitgeberverbandes Alphabetisierungskurse für Fabrikarbeiter durch. FREIRES Konzept einer neuen Alphabetisierungsmethode wurde später unter den Begriffen „Pädagogik der Unterdrückten“ oder „Bildung als Praxis der Freiheit““ weltweit bekannt. In den 50er Jahren begann FREIRE an der Universität Geschichte und 
Philosophie der Erziehung zu lehren. Er initierte erwachsenenbildnerische Aktivitäten, die sich bald im ganzen Land ausweiteten. Anfang der 60er Jahre wurden mithilfe der katholischen Kirche FREIRES Alphabetisierungsprogramme im Radio gesendet. Währenddessen begann im Nordosten Brasiliens eine offizielle Alphabetisierungskampagne. Im Jahr 1963 wurde FREIRES Bildungskonzept auf die nationale Ebene übertragen. Nach dem Staatsstreich von 1964 wurde FREIRES Methode von der neuen Regierung verboten und die Alphabetisierungskurse wurden geschlossen. FREIRE verließ Brasilien, setzte seine Arbeit allerdings im chilenischen Exil fort.

Anfang der 70er Jahre wurde FREIRE Berater für Bildungsfragen in Entwicklungsländern beim Ökumenischen Rat der Kirchen in Genf. Ein Jahrzehnt später konnte er in seine brasilianische Heimat zurückkehren und übernahm Lehrtätigkeiten an verschiedenen Universitäten. Zunehmend engagierte er sich politisch, so war er Mitbegründer der oppositionellen "Partei der Arbeiter" (PT Partido dos Trabalhadores) und arbeitete einige Jahre als "Städtischer Sekretär für Erziehung und Bildung" in São Paulo.

Mit seinen Büchern wurde FREIRE weltweit bekannt. Sein bekanntestes Buch „Pädagogik der Unterdrückten“ (1970) wurde in 18 Sprachen übersetzt. Er wurde als Kandidat für den Friedensnobelpreis vorgeschlagen und mit fast 30 Ehrendoktortiteln ausgezeichnet. In Europa wurden viele pädagogische Bereiche vom Gedankengut PAULO FREIRES beeinflusst, so auch die Erwachsenen- und außerschulische Bildung (WAGNER, 2001).

\section{UNESCO, 1972: Faure-Report}

Die Entwicklung der Erwachsenenbildung in Deutschland steht im Zusammenhang mit internationalen Entwicklungen und Diskursen. Ein besonders wichtiges Forum zur Diskussion und Verbreitung neuer Ansätze ist die UNESCO. Als ein Meilenstein in der Geschichte des lebenslangen Lernens kann hier der sogenannte Faure-Report (FAURE et al., 1972) betrachtet werden. Dieser von der UNESCO im Jahr 1972 unter dem Titel "Learning to be" herausgegebene Report beschreibt die Wichtigkeit des Lernens über die gesamte Lebensspanne hinweg und markierte damit einen Wendepunkt in der internationalen Bildungsdiskussion. 
aspects of education, embracing everything in it, with the whole being more than the sum of its parts. There is no such thing as a separate 'permanent' part of education which is not lifelong. In other words, lifelong education is not an educational system but the principle on which the overall organisation of a system is founded, and which should accordingly underlie the development of each of its component parts. [...] We propose lifelong education as the master concept for educational policies in the years to come for both developed and developing countries. [...]

The various applications of this idea will of course differ greatly. We may even say [...] that it could be applied in as many different ways as there are countries in the world. [...] But we remain convinced that the question of lifelong education, the decisions to take and the paths to follow in order to achieve it are the crucial issues of our time, in all countries of the world, even in those which have yet to become fully aware of this idea. (FAURE et al., 1972: 181-182)

Weiterhin werden Forderungen für die Gestaltung des lebenslangen Bildungsprozesses formuliert. Der Mensch solle nicht einem von außen aufgezwungenen Bildungsauftrag unterworfen werden, sondern durch lebenslanges Lernen die Chance zur Persönlichkeitsentwicklung und zur Wahrnehmung der Welt erhalten. Der Faure-Report beachtet den Menschen in jeder Altersstufe und stellt die Notwendigkeit einer unabschließbaren Bildung und Erziehung heraus. Hier wird über das reine Aneignen von Wissen hinaus ein Schwerpunkt auf das Aneignen von Lernfähigkeit als solcher gelegt.

\begin{abstract}
Education in early childhood should set up the most favourable conditions possible for physical and mental development. Basic education must be many-sided, designed not only for children and adolescents but also for adults who, at any age, may have need of it. [...] Such education aims at learning how to perceive and comprehend the world. It must endeavour to instil, especially in children, a taste for self-learning that will last a lifetime; to arouse their desire to know, to ask questions and to question themselves, while developing the faculties of observation and judgement and the critical spirit. Finally, it should try to awaken the feeling in an individual that he belongs to a community and that each person has a creative responsibility towards himself and others.

The object of post-basic education should be not so much to provide adolescents with access to university as to prepare them for active life [...]. Higher education should be accessible through many different paths and at any age, in many forms, especially with a view to continual self-improvement and development. Occasional education should be available at all ages to suit each person's momentary or permanent needs. " (FAURE et al. 1972: 184)
\end{abstract}

\title{
Deutschland - historischer Abriss der Entwicklung der Erwachsenenbildung
}

Im Mittelalter (bis 1650) gab es noch keine klare Trennung zwischen Lernen und Arbeiten, wie es heute weitgehend der Fall ist. „In den Formen von Gesellenwandern im Handwerk und von Auslandsaufenthalten des Kaufmannsnachwuchses fanden Lernen und Arbeiten weitgehend integriert statt." 
(KELL 1996: 50). Die dreistufige Berufserziehung (Lehrling - Geselle - Meister) begründet sich in der Entwicklung neuer Anforderungen eines existierenden Berufes.

Die Trennung von Lernen und Arbeiten hat in der Epoche des Merkantilismus (1650-1800) ihren Ursprung:

\begin{abstract}
Für die Erwachsenenbildung dieser Zeit ist festzustellen, dass inhaltlich allgemeines und berufliches Lernen noch nicht getrennt und dass die schulisch organisierten Formen noch wenig entwickelt sind, sondern dass autodidaktisches Lernen durch Lesen, zum Beispiel der großen Enzyklopädien und der Fachbücher, der Intelligenz- und Anzeigenblätter und der Fachzeitschriften, vorherrschen. Hinzu kamen die Teilnahme an geselligen Veranstaltungen, Ausstellungen, Kabinetten, Museen usw. Das allgemeine und das berufliche Lernen außerhalb der Arbeit gewinnt inhaltlich an Bedeutung, geht aber zeitlich noch wenig zu Lasten der Lebensarbeitszeit, insbesondere noch nicht in der Erwachsenenbildung. (KELL 1986: 50)
\end{abstract}

Im Zeitalter der Industrialisierung (1800-1918) wurde das Bildungswesen zunehmend als Weg der sozialen Differenzierung und des Aufstiegs angesehen. Die Schülerzahlen vergrößerten sich, die Schulzeiten wurden erweitert und in einzelne Zweige gegliedert. Am Ende des Jahrhunderts war die Dreigliedrigkeit des allgemeinen Schulwesens voll ausgeprägt. Die betriebliche Berufsausbildung nach der Schulzeit wurde durch eine darauf aufbauende Fortbildungsschule ergänzt. Die sich steigernde Zahl der Schulabsolventen führte zu einer wachsenden Wichtigkeit der Weiterbildungsmöglichkeiten. Für die Entwicklung der Erwachsenenbildung waren vor allem die Idee der geschlechtlichen Gleichberechtigung und die technischen und sozialen Neuerungen von Bedeutung. Die Möglichkeiten der Weiterbildung waren allerdings zwischen den sozialen Ständen sehr unterschiedlich.

In der Weimarer Republik (1918-1933) entwickelte sich die Erwachsenenbildung als eine Art nationale Volksbewegung. Dies führte zur Gründung vieler Volkshochschulen und regionalen und überregionalen bildungsbezogenen Zusammenschlüssen. In größeren Städten wurden sogenannte Volksbildungsämter eingerichtet, die die Bildungseinrichtungen koordinieren und um weitere Bildungsmöglichkeiten erweitern sollten. Durch die Revolution von 1848/49 war die politische Gleichberechtigung der wirtschaftlich Unterprivilegierten vorangetrieben worden, so dass nun oftmals die Arbeiter als Zielgruppe der Bildungsentwicklung im Vordergrund standen. 
„Zwischen der Volksbildung als Idee von der Bildung des ganzen Volkes und der Realität im Bildungssystem bestanden jedoch erhebliche Differenzen." (KELL 1996: 52). Es hatte sich ein gestuftes Bildungswesen entwickelt, das der Hierarchie der beruflichen Tätigkeiten und den dafür erforderlichen Qualifikationen angepasst war. „Mit dem Demokratieverständnis und den politischen Postulaten von Freiheit und Gleichheit stand eine Isolierung dieser ausdifferenzierten Bildungs- und Berufswege nicht im Einklang. Deshalb war dem Prinzip der Durchlässigkeit durch Integrationsmaßnahmen Geltung zu verschaffen. Dahingehende Bemühungen sind die Ursprünge [...] für die Entwicklung des "Zweiten Bildungsweges“.” (KELL 1996: 52). In den Vordergrund trat nun die Möglichkeit des Nachholens der allgemeinen Berechtigungen für die Bildungsgänge. Mit dieser Aufgabe waren vor allem die Volkshochschulen beschäftigt, wobei ein besonderes Augenmerk auf dem Nachholen des Abiturs lag.

Anfang der 20er Jahre wurden erstmals sogenannte "Arbeiterkurse" in einigen Großstädten eingerichtet. Aus diesen entwickelten sich speziellere Weiterbildungseinrichtungen wie Abendgymnasien, Abendrealschulen oder Berufsaufbauschulen.

Nach der Zeit des Nationalsozialismus und dem Zweiten Weltkrieg (19331945) wurde die Struktur des Bildungssystems wieder so ähnlich aufgebaut, wie sie sich in der Weimarer Republik entwickelt hatte. Im Nationalsozialismus lag das Hauptaugenmerk des Bildungssystems auf der nationalsozialistischen Erziehung. In den Umerziehungsprogrammen der alliierten Siegermächte nach 1945 kam der Erwachsenenbildung eine große Bedeutung zu.

\section{Jüngere Bildungspolitik in Deutschland}

In Deutschland gab es im letzten Jahrzehnt einige interessante Entwicklungen im bildungspolitischen Bereich. Im Jahr 2001 kam eine auf Beschluss des Bundestages eingesetzte unabhängige Expertenkommission zusammen, die Vorschläge zur Finanzierung lebenslangen Lernens erarbeiten sollte. Indirekt sollten die Vorschläge der Kommission ebenfalls darauf ausgerichtet sein, die Bildungsbeteiligung in Deutschland zu erhöhen, Anreize für lebenslanges Lernen zu schaffen und die Förderung aller Begabungen zu verbessern. 
Lebenslanges Lernen umfasst im Verständnis der Kommission die Gesamtheit allen formalen, non formalen und informellen Lernens über den gesamten Lebenszyklus eines Menschen hinweg. In der praktischen Ausgestaltung sollen bisher stark segmentierte Bildungsbereiche stärker verzahnt werden. Lernangebote sollen inhaltlich, zeitlich und räumlich derart miteinander verkoppelt sein, dass die Lerner für sich systematische, durchlässige und zertifizierbare Lernprozesse gestalten können. (EXPERTENKOMMISSION FINANZIERUNG LEBENSLANGEN LERNENS 2004: V)

In ihrem Abschlussbericht im Jahr 2004 beschreibt die Kommission die Notwendigkeit der bildungspolitischen / staatlichen Verankerung Lebenslangen Lernens:

Die Eigenverantwortung für Lebenslanges Lernen steigt im Erwachsenenalter. Die elterliche Verantwortung im Lernprozess der Kinder wird im Erwachsenenlernen durch die Eigen- und Selbstverantwortung der Lerner abgelöst. (...) Auch die öffentliche Verantwortung für Lebenslanges Lernen steigt in einer Wissensgesellschaft. Die Kommission geht davon aus, dass der Staat jedem Bürger freien Zutritt zu einem bestimmten Niveau an Allgemeinbildung und zu einer beruflichen Erstausbildung gewährleistet. Viele Erwachsene verfügen aber nicht über diese Basisqualifikationen. Daher beschränkt sich die öffentliche Verantwortung nicht mehr nur auf die Jugendphase, sondern muss sich auch auf das Erwachsenenalter erstrecken. Gleichzeitig hat der Staat die Aufgabe, durch die Gewährung von Mitteln für berufliche Bildung von Personen mit geringem Einkommen und Vermögen zu unterstützen [...].(EXPERTENKOMMISSION FinANZIERUNG LEBENSLANGEN LERNENS, 2004: X)

Das langfristige Ziel der Kommission zur Finanzierung lebenslangen Lernens sei ein einheitliches Bildungsförderungsgesetz, in dem alle Leistungen der Förderung erwachsener Lerner zusammengefasst werden. Besonders das Nachholen von Schul-, Hochschul- und Berufsbildungsabschlüssen solle durch ein gestuftes System von staatlichen Zuschüssen oder Darlehen ermöglicht werden. $\mathrm{Da}$ die deutsche Gesellschaft in den nächsten Jahrzehnten durch einen Geburtenrückgang, stabile Sterblichkeitsraten und steigende Lebenserwartung deutlich altern wird, befürchtet die Kommission ein Veralten des bisher akkumulierten Wissens und einen verlangsamten Zufluss neuen Wissens. Dem soll durch die ständige Möglichkeit der Weiterbildung entgegengewirkt werden.

\footnotetext{
Um das in Betrieben und anderen Organisationen erforderliche Wissen zu erneuern, wird man immer weniger auf die bisherigen Muster des Personalaustausches durch Frühpensionierung und Einstellung jüngerer Arbeitnehmer zurückgreifen können. Die Erneuerung muss in zunehmendem Maße durch das Lebenslange Lernen der Erwerbstätigen und damit auch der älteren Arbeitnehmer getragen werden. [...] Es gilt anzuerkennen, dass Ältere weiterlernen und produktiv bleiben können. (EXPERTENKOMMISSION FINANZIERUNG LEBENSLANGEN LERNENS, 2004, S. 28)
}

Eine Analyse des Sozioökonomischen Panels (SOEP) von Daten im 
PANNENBERG, 2004). Relevante Ergebnisse der Befragungen werden im Folgenden dargestellt:

„Lernen nutzt der beruflichen Situation“: $70 \%$ bis $90 \%$ der Befragten gaben an, dass sich die berufliche Weiterbildungsmaßnahme für sie gelohnt habe (beispielsweise in den Bereichen Einkommen, Karriere, Arbeitsplatzsicherung etc.).

"Lernen zahlt sich aus": Für Westdeutschland wurde in der Altersgruppe der 20- bis 44-Jährigen eine durchschnittliche Erhöhung des Bruttomonatseinkommens nach einer Weiterbildungsmaßnahme in Höhe von 4,5\% ausgewiesen. In der Gruppe der 45- bis 64-Jährigen lag keine Steigerung vor. In Ostdeutschland erhöhte sich das durchschnittliche Bruttomonatseinkommen für beide Gruppen signifikant aufgrund einer Weiterbildungsteilnahme (7\% bzw. $8 \%$ ).

„Lernen fördert die berufliche Entwicklung“: Bei westdeutschen Männern stieg die Chance auf einen beruflichen Aufstieg durch eine Weiterbildungsmaßnahme um zwei Prozentpunkte.

„Lernen mindert das Risiko von Arbeitslosigkeit": Teilnehmer an einer Weiterbildungsmaßnahme wiesen insgesamt ein deutlich niedrigeres Risiko auf, zukünftig arbeitslos zu werden. In der Altersgruppe der 20- bis 44Jährigen reduzierte sich das Risiko durch die Weiterbildung um $2 \%$ (West) bis $5 \%$ (Ost).

Das Konzept des Lebenslangen Lernens stellt auch in der jüngsten Zeit ein wichtiges Thema für die deutsche Bildungspolitik dar. Im September 2008 stellt die Partei „Die Grünen“ Eckpunkte für das sogenannte „Erwachsenen-BAFöG“, ein Bundesgesetz zur Förderung der individuellen Fort- und Weiterbildung vor (BÜNDNIS 90 - DiE GRÜNEN, 2008).

Sinn des Gesetzes ist u.a. die Weiterbildungsbeteiligung derjenigen zu steigern, die bisherkaum teilnehmen. Dies sind neben älteren Arbeitnehmerlnnen v.a. Geringqualifizierte, Frauen nach der Familienphase und Menschen mit Migrationshintergrund. Daraus folgt, dass für den Förderanspruch keine starren Altersregelungen gelten, dass die aufenthaltsrechtlichen Anforderungen gering gehalten sind und dass Teilzeitbeschäftigte und WiedereinsteigerInnen gleiche Förderansprüche wie Vollzeitbeschäftigte haben. Eine solche Förderung entzerrt die "rush-hour", weil sie mehr Zeit für Bildung gibt. Dadurch wird es möglich, die Lebensphase des Berufseinstiegs, der Familiengründung und der ökonomischen Selbständigkeit flexibler selbst zu gestalten. (BÜNDNIS 90 - DIE GRÜNEN, 2008). 
Gefördert werden sollen laut Gesetzesvorschlag alle Fort- und Weiterbildungen, die der beruflichen Weiterentwirklung dienen, wobei das primäre Ziel das Erreichen staatlich zertifizierter Abschlüsse sei. Das Nachholen von Schulabschlüssen solle altersunabhängig komplett staatlich finanziert werden; Förderungen beruflicher Weiterbildungen würden sich aus Darlehen und Zuschüssen zusammensetzen.

„Eine autonome Lebensführung und verantwortungsbewusste Teilhabe an der Gesellschaft sind ohne Bildung nicht denkbar. Bildung ist daher eine gesamtgesellschaftliche Aufgabe.“ (BMFSFJ 2002: 6) Diese Einschätzung seitens des Bundesministeriums ist sachlich zutreffend." (MERTEN 2006: 65)

Als zwei wichtige Voraussetzungen für ein erfolgreiches lebenslanges Lernen, lassen sich in der Literatur durchweg zudem folgende Aspekte finden:

Motivation und Interesse bzw. Wertschätzung für Bildung und Lernen sowie

die Kompetenzen, um diese Bildungsmotivation auch erfolgreich umsetzen zu können (ACHTENHAGEN/LEMPERT 2000). [...]

Übereinstimmung herrscht in der Literatur auch darin, dass Lernkompetenzen langfristig notwendig und erforderlich sind, um das Interesse am Lernen aufrecht zu erhalten. Nur wenn man sich und sein Lernen reflektieren und steuern kann, ist man auf lange Sicht erfolgreich dabei. Sich als erfolgreich und selbstbestimmt bei einer Sache zu erleben ist die Voraussetzung dafür, intrinsische Motivation bzw. Interesse langfristig zu erhalten (s. auch SPIEL/SCHOBER 2002: 86). Nach SPIEL/SCHOBER durchläuft zudem jeder Lernende vier Phasen: die Abwägphase, die Planungsphase, die Handlungsphase und schließlich die Bewertungsphase, wie sie folgende Grafik darstellt:

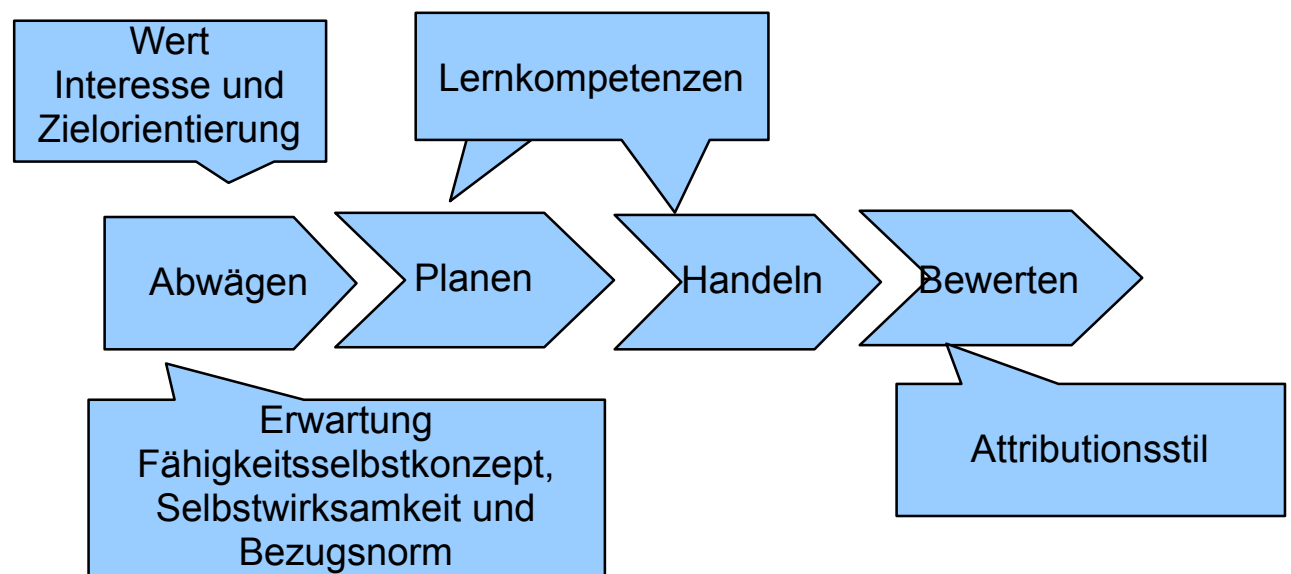

Abb. 1 Vier Phasen des Lernenden (vgl. SPIEL 2002) 
Aus dem Kontext der Schule und Hochschule entwickelte SPIEL einige Forderungen, zur Förderung von Lernprozessen. Einige davon werden im Fortgang der Arbeit nochmals vertiefend und kongruent mit aktuellen Ergebnissen aus der Hirnforschung dargestellt:

\begin{abstract}
Abschließend werden, basierend auf den Befunden der einschlägigen internationalen Literatur, den Forderungen der Bildungspolitik und nicht zuletzt auf den Ergebnissen der präsentieren Studien Forderungen für Schule und Hochschule abgeleitet, deren Realisierung die systematische Förderungen von Bildungsmotivation und Kompetenzen zum LLL voranbringen sollte:
\end{abstract}

- frühe Förderung und gezielte Stützung der positiven motivationalen Ausgangslage in den ersten Schuljahren bzw. in der Vorschule,

- verstärkende Berücksichtigung der Interessen der Lernenden und damit auch verstärkte Individualisierung,

- $\quad$ selbstgesteuertes Lernen, bei dem gelernt wird, Verantwortung für das eigene Lernen zu übernehmen, sollte zu einem eigenem Lehrziel gemacht werden (in Schule und Hochschule),

- Forcierung von Lernen in Gruppen und an Projekten,

- $\quad$ stärkere Betonung von Kompetenzereweiterung als Ziel des Lernens - damit weg von einer reinen Performanzorientierung („Sammeln von Scheinen“),

- motivationsförderliches Leistungsfeedback geben und auch annehmen lernen,

- Akzeptanz und Übernahme der Ergebnisverantwortlichkeit bei allen Beteiligten (Lehrpersonen, Lernende, Eltern, Schulaufsicht, etc.),

- $\quad$ die Kompetenz zur Motivationsförderung sollte einen größeren Raum in der Aus- und Weiterbildung sowie in der Kommunikation zwischen Lehrpersonen einnehmen. (SPIEL 2006: 95)

Ohne eine Defizitfokussierung vorzunehmen, sollte zu obigen Aspekten zusätzlich zwingend die Identität alter Migranten Berücksichtigung finden. Große Bedeutung haben - und sollten daher einbezogen werden - die vorhandenen Ressourcen:

Gerade für die praktische Arbeit von Sozialarbeitern in den Betreuungsinstitutionen sowie für die der Politiker und Politikerinnen und Gesetzgeber sei Wissen über die Identität älterer Menschen wichtig, um nicht Abhängigkeit und Schwächen dieser Gruppe zu verstärken, sondern Unabhängigkeit und Aktivitäten zu fördern (vgl. auch PAOLETTI 2001). (GLASER UND RENTORFF 2006: 176)

\title{
Ebenso formulieren die Autoren auf kommunalpolitischer Ebene
}

Umsetzungswünsche, die nicht unbeachtet bleiben dürfen:

- Stärkung der Eigenverantwortung und Selbststeuerung der Lernenden,

- Motivierung benachteiligter bzw. bildungsferner Gruppen,

- Stärkung der Bezüge zwischen allen Bildungsbereichen,

- Kooperation der Bildungsanbietenden sowie Nutzer und Nutzerinnen,

- qualitative und quantitative Verbesserung der Angebote und Angebotsstruktur, vor allem im Sinne einer stärkeren Nutzerorientierung (BMBF 2004). (TIPPELT et al. 2006: 281) 
Lebenslanges Lernen wird von WULF UND ZIRFAß (2006) als breitgefächerter, nicht auf Rituale und Ritualisierungen beschränkt charakterisierte Erziehung und Bildung beschrieben, sondern aus einer performativen Sichtweise gesehen:

\begin{abstract}
Unter dem Blickwinkel der Performativen erscheinen unter dem Titel „Bildung“ nicht nur spezifische soziale bzw. individuelle habituelle Prägungen, sondern vor allem auch Bildungsprozesse als Lern-, Handlungs- und Veränderungsprozesse in deren Vollzügen eine Formung und Leistung des Bildenden zur Geltung kommt. In Bildungsprozessen lassen sich Differenzen in zeitlicher, identifikatorischer, institutioneller oder sozialer Hinsicht festmachen; sie sind als Differenzgeschehnisse Übergangsphänomene, deren Dezentrierungsmomente nicht selten durch Erziehung und Sozialisation expliziert, kanalisiert und finalisiert werden. Das Bildungspotential performativer Prozesse liegt in ihren kreativen und wirklichkeitserzeugenden Momenten, die Dispositionen und Disponibilitäten der Beteiligten hervorbringen können. Im performativen Fokus wird der Begriff der Bildung erweitert; denn das reflexive Potential der traditionellen Bestimmung des Begriffs wird beibehalten und um die Bildungsprozesse ergänzt, die nicht nur als kognitive, sondern auch als körperliche, soziale, situative und inszenierte Prozesse verstanden werden. (WULF UND ZIRFAß 2006: 298f)
\end{abstract}

Die zusätzliche Einbeziehung der sozialen, situativen und insbesondere der 'inszenierten' Prozesse durch WULF UND ZIRFAß - zumal diese sich auch in die Zukunft orientieren können - führen zum folgenden Ansatz des antizipatorischen Lernens.

\title{
3.2.2 Antizipatorisches Lernen
}

Ein weiterer wichtiger Ansatz im Zusammenhang mit Lernen darf keinesfalls vernachlässigt werden. Die Rede ist vom 'antizipatorischen Lernen' nach HALLER und FleCHSIG (2009). Hierbei wird Wissen als Generierung über zukünftige Verhältnisse bzw. auch zur Bewältigung zukünftiger Verhältnisse verstanden, wie sie HALLER (2011) ${ }^{43}$ sehr trefflich formuliert hat:

Das Besondere am Konzept des antizipatorischen (vorwegnehmenden) Lernens ist in der Gegenüberstellung zum gewissermaßen klassischen Lernbegriff zu sehen, der als adaptives Lernen bezeichnet werden kann, bei dem es um ein Lernen als Übernahme vorhandenen Wissens geht, als Einbindung in einen bereits bestehenden Kanon des in vorherigen Generationen angesammelten Wissens. Demgegenüber wird antizipatorisches Lernen verstanden als Generierung von Wissen über zukünftige Verhältnisse bzw. auch zur Bewältigung zukünftiger Verhältnisse."

$[\ldots]$

Karl-Heinz Flechsig hat 2009 in einer seiner letzten Arbeiten das Konzept des antizipatorischen Lernens noch einmal aufgegriffen und weiter entwickelt. Unerklärlicher Weise ist die Abhandlung des "Club of Rome" jedoch in Deutschland ansonsten kaum rezipiert worden. So weist Google (am 25.11.2010) 45 Einträge für „antizipatorisches

43 HANS-Dieter HALLER, Über antizipatorisches und partizipatorisches Lernen: ein Diskussionsbeitrag für die Mitwirkenden am Projekt "Inszenierte Bildung- Denk(t)räume“, Unveröffentlicht: freundlicherweise persönlich zur Verfügung gestellt am: 02.03.2011 
Lernen“, 1070 für „anticipatory learning“, indessen 6520 für „Grenzen des Wachstums“ und 38500 für "limits to growth" auf.

Die in der Abhandlung "Das menschliche Dilemma“ aufgezeigten Gründe, warum innovatives Lernen gerade für die Gegenwart notwendig sei, und zwar in dramatischem Ausmaß, verweisen auf die ökologische Krise, über die seit den ausgehenden1970er Jahren gesprochen wird: Umweltverschmutzung, Waldsterben, Klimakatastrophe u.a. sind drohende Folgen von ungezügelter Produktion und Lebensweise, die man erwarten muss, aber deren Art und weitere Folgen nicht kalkulierbar sind. Die Menschheit muss lernen, was sie noch nicht kennt, muss reagieren, worauf sie noch nicht vorbereitet ist.

Nun muss allerdings auch berücksichtigt werden, dass gerade für Kinder und Jugendliche formelles (also schulisches) Lernen in hohem Maße antizipatorisch ist, gewissermaßen ein Scheck auf ihre Zukunft. „Nicht für die Schule, sondern für das Leben lernen wir", so wurde das ursprüngliche "non vitae, sed scholae discimus" verdreht ${ }^{44}$, oder wie Paolo Freire gesagt hat, schulisches Lernen ist eine Einlage in eine Bank, die später einmal ausbezahlt werden soll. Der Lehrer mache Einlagen in die Köpfe der Schüler. Es sei seine Aufgabe, die Köpfe der Schüler "mit den Inhalten seiner Übermittlung zu füllen - mit Inhalten, die von der Wirklichkeit losgelöst sind, ohne Verbindung zu einem größeren Ganzen, das sie ins Leben rief und innen Bedeutung verleihen könnte" (Freire 1973, S.60). "Je vollständiger er die Behälter fülle, ein desto besserer Lehrer ist er. Je williger die Behälter es zulassen, dass sie gefüllt werden, um so bessere Schüler sind sie" (Freire 1973, S. 57). Das aber mache die Schüler passiv. Sie nähmen die innen präsentierte Welt hin und passten sich der scheinbaren Realität an. "Das Bankiers-Konzept beruht auf der Voraussetzung einer Spaltung zwischen Mensch und Welt: der Mensch ist nur in der Welt, aber nicht mit der Welt oder mit anderen. Der Mensch ist Zuschauer, nicht Neuschöpfer. In dieser Sicht ist der Mensch nicht ein bewusstes Wesen, vielmehr ist er Besitzer eines Bewusstseins: eines leeren Sinnes, der dem Empfang von Einlagen an Wirklichkeit aus der Außenwelt passiv offen steht" (Freire1973, S. 57)." [...]

Es ist naheliegend, im Hinblick auf unsere Frage nach der Zukunftsorientierung im Lernen von Kindern, Jugendlichen und Erwachsenen auch danach zu fragen, wie das Konzept von Zukunft überhaupt im kulturellen und sozialen Zusammenhang und speziell bei diesen Altersgruppen beschaffen ist. (Haller 2011: 2-3)

Die Berücksichtigung der kulturellen und sozialen Zusammenhänge des antizipatorischen Lernens bezogen auf das Alter bzw. auf das Älterwerden fordert nach Erklärung, wie Lernen und Wissenserwerb außerhalb von Bildungseinrichtungen stattfinden kann. Hierzu wird im folgenden Abschnitt eingegangen.

\subsubsection{Formelles vs. informelles Lernen}

In heutigen Gesellschaften herrscht ein ständiger Wandel. Die Globalisierung, moderne Technologien und die Massenmedien bringen für die Menschen große Veränderungen mit sich, denen sie nur durch ständige Aktualisierungen ihres Wissens und ihrer Fertigkeiten begegnen können. „Alle Menschen lernen - bewusst und unbewusst - ihr Leben lang“ (DOHMEN, 2001). Wissen wird als die wichtigste Grundlage für Weiterentwicklung angesehen. Wer

44 Seneca, epistulae morales ad Lucilium 106, 12 
nicht an der Bildung teilnimmt, wird mehr und mehr von der Teilnahme an der Gesellschaft ausgeschlossen. Dies erfordert lebenslanges Lernen in verschiedenen Bereichen und durch verschiedene Lernprozesse, die im Folgenden erläutert werden sollen.

Mit dem Begriff des formellen Lernens bezeichnet man das Lernen an Bildungs- und Ausbildungseinrichtungen wie Schulen oder Universitäten. Synonym werden hier die Ausdrücke formales, direktes oder intentionales Lernen verwendet. Ein Beispiel für die Inhalte eines solchen Lernprozesses sind mathematische Algorithmen oder Vokabeln im Sprachunterricht. Das formelle Lernen führt zu anerkannten Abschlüssen oder Qualifikationen wie dem Abitur oder Diplomzeugnis.

Doch nicht nur formelles, sondern auch besonders der Prozess des Informellen Lernens (auch indirektes oder inzidentelles Lernen genannt) ist sehr wichtig für die menschliche Gesellschaft. Dieser Begriff beschreibt „Lernprozesse, die ihren Platz außerhalb formaler Institutionen haben“ (WINKLER UND MANDL, 2005). Diese Form des Lernens kann in der Familie, im Beruf oder in der Freizeit stattfinden und führt nicht zum Erwerb eines Abschlusses. REBER (1967) beschrieb das informelle Lernen als einen „Prozess, durch den ein Organismus Wissen über Regelmäßigkeiten einer komplexen Umwelt erwirbt, ohne dies intendiert zu haben und ohne sich größtenteils dessen bewusst zu sein, was er lernt.“

„Da etwa 70\% aller menschlichen Lernprozesse außerhalb der Bildungsinstitutionen stattfinden, da aber meist nur die schulischen Lernerfolge gesellschaftlich anerkannt und honoriert werden, gibt es eine ungerechtfertigte gesellschaftliche Bildungskluft aufgrund schulischer Zeugnisse und „Begabungen“. Die Begabungen und Kompetenzen, die sich als Ergebnisse des informellen Lernens außerhalb des autorisierten Bildungswesens entwickeln, finden nicht die Beachtung und Anerkennung, die sie verdienen." DOHMEN (2001:7)

DOHMEN (2001) erklärt, dass das formelle Lernen eine Ergänzung für das informelle Lernen darstellen sollte. Problematisch ist seiner Meinung nach, dass ursprünglich neugierige und wissbegierige Schüler im Laufe ihrer Schullaufbahn oft das eigene Interesse am Lernen verlören, da für sie aus nicht nachvollziehbaren Gründen oft sehr theoretische Inhalte gelehrt würden. Das 
fehlende Interesse bei den Lernenden würde nur durch Sanktionen, Belohnungen und die Vergabe gesellschaftlicher Berechtigungen kompensiert. WATKINS und MARSICK (1992) nennen dies "theoretisches Lernen ohne Handeln“ und bezeichnen es als Kennzeichen des formellen Lernens (s. Tabelle unten). Das formelle Lernen solle dergestalt verändert werden, dass die intrinsische Lernmotivation des Schülers gefördert und nicht abgebaut wird. Lernen sei nur dann wirklich interessant und bildungswirksam, wenn es von den Lernenden als so wichtig und hilfreich erfahren wird wie das informelle Lernen in alltäglichen Umweltsituationen.

Tabelle 3 Charakteristika des formellen und informellen Lernens nach WATKINS UND MARSICK (1992)

\begin{tabular}{|l|l|}
\hline formelles Lernen & „Reflection without Action“ \\
\hline $\begin{array}{l}\text { informelles Lernen } \\
\text { inzidentelles Lernen }\end{array}$ & "Action with Reflection“ \\
\hline Nicht-Lernen & „Absence of Action and \\
& Reflection“ \\
\hline
\end{tabular}

Am Beispiel traditioneller afrikanischer Kulturen kann die Bedeutung des informellen Lernens aufgezeigt werden. Dass es in diesen Kulturen teilweise keine Schulen gibt, bedeutet nicht, dass die Kinder nicht gelehrt werden. FAURE ET AL. (1972) erklärt, dass die Bildung dort komplex und kontinuierlich sei. Sie ziele darauf $\mathrm{ab}$, den kindlichen Charakter zu formen, Haltungen, Fertigkeiten und moralische Wertvorstellungen auszubilden. Dies geschieht nicht durch Bildung im schulischen Sinne, sondern im Prozess der Aufnahme in die Gesellschaft. Das Leben im Familienkontext, die körperliche Arbeit oder religiöse Zeremonien böten täglich Möglichkeiten, etwas zu lernen. Das informelle Lernen geschehe beispielsweise im Kontakt mit der fürsorglichen Mutter, bei den Jagdausflügen mit dem Vater, bei der Beobachtung von Tieren und des Wechsels der Jahreszeiten oder wenn die Großeltern Sagen und Märchen erzählten.

Informell erworbenes Wissen sei auch für das Lernen im schulischen Kontext wichtig, indem es den Lernenden darauf vorbereite, neu Gelerntes zu ordnen und zu konzeptualisieren. So kann zum Beispiel die hohe Alphabetisierungsrate von $98,5 \%$ auf den Malediven teilweise dadurch erklärt werden, dass dort kleine Kinder unabhängig von einer bevorstehenden 
Schulbildung zu einer älteren Lehrerin geschickt werden, die ihnen Unterricht in traditioneller Kultur erteilt.

Das „informelle Lernen ist aber ein bisher von der deutschen Bildungspolitik, Bildungsforschung und Bildungspraxis weitgehend vernachlässigtes Feld. Die pädagogischen Interessen haben sich in Deutschland bislang vorwiegend auf das in Schulen und Hochschulen institutionalisierte Lernen - und damit nur auf die Spitze eines sehr viel umfangreicheren „Eisbergs“ menschlichen Lernens - konzentriert. Das hängt damit zusammen, dass das meist mit anderen Lebenstätigkeiten verbundene informelle Lernen schwerer zugänglich, faßbar und beeinflußbar ist als das geregelte Lernen in dafür eingerichteten Bildungsinstitutionen“. DOHMEN (2001: 3)

Über die genaue Bedeutung und Abgrenzung des Begriffs des informellen Lernens gibt es daher keinen eindeutigen Konsens. Teilweise wird informelles Lernen mit inzidentellem Lernen gleichgesetzt DEWEY (1997), andere Autoren betrachten inzidentelles Lernen als einen Teil des informellen Lernens WATKINS UND MARSICK (1990), wieder andere grenzen beide Lernprozesse voneinander ab DOHMEN (1996).

Im Folgenden wird die Darstellung von WINKLER UND MANDL benutzt, die zur besseren Übersichtlichkeit grafisch veranschaulicht wurde (s.u.). Die Autoren unterteilen informelles Lernen in Reflexives Erfahrungslernen und Implizites Lernen. Das Ergebnis dieser beiden Vorgänge ist das sogenannte Erfahrungswissen. 


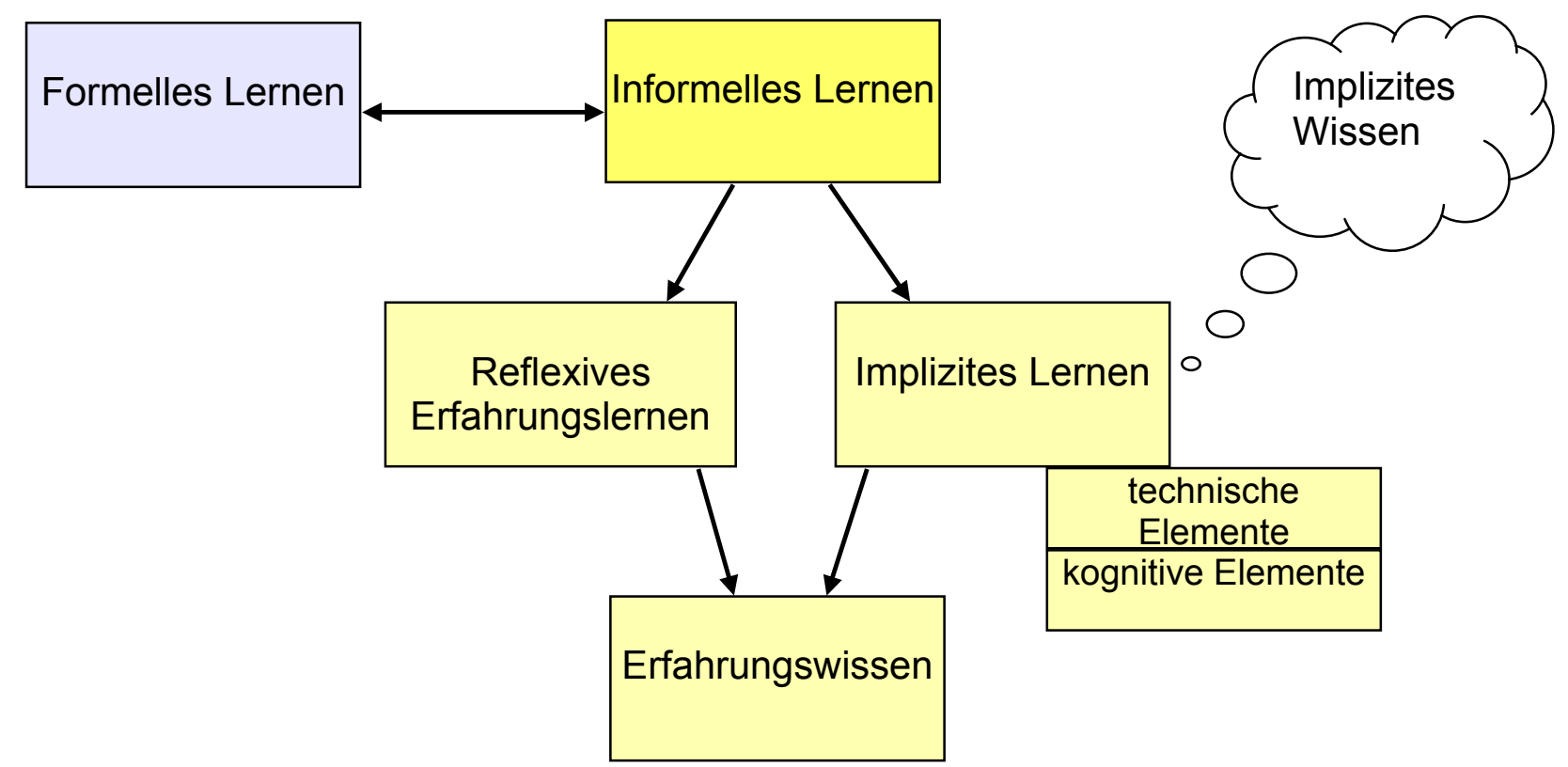

Abb. 2 Modell der Unterteilung verschiedener Lernprozesse nach WINKLER UND MANDL (2005)

Durch Rahmenbedingungen die auf der einen Seite z.B. niedrigschwelliges Angebote wie das antizipatorische Lernen und andererseits die Selbststeuerung/organisation zulassen, kann das informelle Lernen unterstützt werden:

Das meiste Lernen ist nicht das Ergebnis von Unterweisung. Es ist vielmehr das Ergebnis ungehinderter Teilnahme in sinnvoller Umgebung. (Illich 1973) [...] Didaktische Maßnahmen würden also darin liegen, die Rahmenbedingungen so zu setzen bzw. die Lernumgebung so zu konzipieren, dass möglichst viele Kriterien des Lernprozesses nicht eindeutig festgelegt sind. [...] Schließlich ist der Zugang zum Lernen von Interesse: Da informelles Lernen oft ungeplant und beiläufig vor sich geht, hat man es hier mit niederschwelligen Zugängen zu tun. Will man daher informelles Lernen fördern, sind solche Zugänge einzurichten. (ZÜRCHER 2007: 47)

\section{Beim reflexiven Erfahrungslernen werden Erfahrungen bewusst} reflektiert, der Lernprozess wird rückgekoppelt und durch die Evaluierung intensiver verarbeitet. Dabei sollen durch das in Beziehung Setzen spezifischer Lehr- und Lernsituationen mit subjektiven Theorien Reflexionsfähigkeit bzw. Reflexionswissen erzielt werden. Ein Beispiel für einen solchen Prozess wird mit der kollegialen Fallberatung von WILDT (1996) anschaulich gemacht:

Eine solche Beratung verläuft in der Regel so, daß ein Mitglied einer kollegialen Gruppe über einen erlebten Fall aus der eigenen Praxis erzählt. In einer Nachfragephase hat die Gruppe Gelegenheit, ihr Bild von dem erzählten Fall zu vervollständigen. Zunächst steht dabei die Sicht des Falles aus der Perspektive der Erzählenden im Mittelpunkt. Erst dann wird dazu übergegangen, den Fall im Spiegel der Gruppe zu betrachten. Es wird dabei geprüft, welche Bedeutung das Verständnis des Falles übrigen Gruppenmitglieder besitzt, die als im selben bzw. vergleichbaren Praxisfeld Tätige über vergleichbare Erfahrungen verfügen. Erst in einem weiteren Schritt wird dann versucht, die zur Sprache gebrachten 
Erfahrungen generalisierend zu deuten, um abschließend zu erörtern, welche Handlungsalternativen gesehen werden und wie diese im Hinblick auf ihre Angemessenheit von den Beteiligten beurteilt werden. Auf dieser letzten Stufe besteht dann die Möglichkeit, Handlungs- und wissenschaftliches Wissen in Bezug zu setzen sowie beide Urteilsformen aufeinander zu beziehen, ohne sie miteinander zu vermischen. WILDT (1996: 7f) ${ }^{45}$

Das implizite Lernen dagegen geschieht ganz unreflektiert und unbewusst. Es findet sich in vielen Bereichen und ist eng mit praktischen Erfahrungen verknüpft. „Implizites Lernen bezieht sich - wie in der Regel auch das informelle Lernen - nicht auf Regelkenntnis, sondern auf eine mehr gefühlsmäßigganzheitliche Reizaufnahme, Situationserfassung, Gestaltwahrnehmung, die jeweils nicht zu explizitem Wissen führt, sondern mehr im Bereich von Intuition und Gespür, Einfühlung und Improvisation bleibt.“ DOHMEN (2001: 34)

Das implizite Lernen birgt nach DOHMEN (2001) die Gefahr von Irrtümern, Vorurteilen und Missverständnissen, solange es nicht bewusst reflektiert und nicht kritisch evaluiert wird. Deshalb solle man sich auch das zunächst unbewusste implizite Lernen im Nachhinein als Lernvorgang bewusst machen.

Das sogenannte implizite Wissen eines Menschen entsteht zu einem großen Teil durch implizites Lernen. POLANYI (1967)

Implizites Wissen zeichnet sich dadurch aus, dass man etwas weiß, es aber nicht gut sprachlich kommunizieren oder erklären kann. Ein Beispiel dafür ist das Fahrradfahren - niemand kann gut erklären, warum er nicht mitsamt dem Rad umfällt oder wie er es schafft, das Gleichgewicht zu halten.

Nach CHAMOuX (1986) kann man darunter das Wissen verstehen, was man im Zusammenleben einer Gemeinschaft und durch die Teilhabe an sozialen Aktivitäten erwerben kann. Meistens ist es kontextspezifisch, das heißt das dadurch erworbene Wissen kann nicht auf andere Bereiche angewendet werden. In einer Studie konnte gezeigt werden, dass die im Zusammenhang des Webens von Stoffen gelernten geometrischen Muster nicht auf geometrische Aufgaben in der Schule übertragen werden konnten GREENFIELD UND LAVE (1982).

\footnotetext{
${ }^{45}$ Johannes Wildt: Reflexives Lernen: http://www.u-asta.unifreiburg.de/engagement/referate/lehramt-referat/Grundsatzpapiere/wildt, Letzter Zugriff: 17.06.2011
} 
NONAKA und TAKEUCHI (1997) unterteilen den Bereich des impliziten Wissens in technische und kognitive Elemente. Die technischen Elemente beinhalten Knowhow, handwerkliches Geschick und Fertigkeiten, während der kognitive Bereich "mentale Modelle“ enthält, die die Grundlage für die individuelle Wahrnehmung der Welt durch den Menschen darstellen. Darunter fallen Paradigmen, Wertesysteme, Vorstellungen und Überzeugungen.

Zusammengefasst lässt sich sagen, dass implizites Lernen durch fehlende Intentionalität, d.h. es ist nicht bewusst auf ein bestimmtes Ergebnis gerichtet, unbewusste Aufmerksamkeitszuwendung und eine eingeschränkte Verfügbarkeit des Gelernten, gekennzeichnet ist.

Das meiste, was Individuen über die Kultur und Traditionen ihrer Gesellschaft wissen, haben sie durch tägliche außerschulische Erfahrungen gelernt. „Erfahrungen entstehen aus der aufmerksamen Wahrnehmung von Reizstrukturen, Sinneseindrücken, Erlebnissen, Begegnungen etc. aus der Umwelt und ihrer persönlichen Verarbeitung. Der Mensch macht Erfahrungen, d.h. er nimmt etwas, was inm begegnet - was aus seiner Umwelt auf inn zukommt, was er hört, sieht, empfindet, erlebt - selektiv auf und bezieht es aktiv ein in den Zusammenhang dessen, was er bisher schon wahrgenommen - „als wahr aufgenommen" und erfahren [...] hat. Das heißt: Erfahrungen sind nicht einfach Produkte oder Reflexe der Umwelt, sondern Ergebnisse eines Zusammentreffens und einer [...] Auseinandersetzung zwischen Person und Umwelt." DOHMEN (2001: 27f.)

Informelles Lernen beinhaltet somit die Aneignung von Fertigkeiten (Skills) durch Erfahrungen. Zu diesen sogenannten Life-Skills gehören das Lernen von Werten und Haltungen für das Zurechtkommen mit Anforderungen der Umwelt, kritisches Denken, Problemlösen, Kommunikation, Toleranz und Offenheit für Veränderungen. „Das heißt: Informelles Lernen ist ein instrumentelles Lernen, ein Mittel zum Zweck. Der Zweck ist - im Gegensatz zum formalen Lernen - nicht das Lernen selbst, sondern die bessere Lösung einer außerschulischen Aufgabe, einer Situationsanforderung, eines Lebensproblems mit Hilfe des Lernens." DOHMEN (2001: 19) 
LEADBEATER $(2000)^{46}$ verdeutlicht dies folgendermaßen: „Der Sinn von Bildung sollte nicht die Entwicklung eines großes Wissensschatzes sein, sondern die Entwicklung von Fähigkeiten: Ebenso Basisfähigkeiten wie die Kenntnis des Lesens, Schreibens und Rechnens wie die Fähigkeit, sich verantwortungsvoll gegenüber Anderen $\mathrm{zu}$ verhalten, Initiative $\mathrm{zu}$ ergreifen und kreativ und kollaborativ zu arbeiten. Die wichtigste Fähigkeit, die die traditionelle Bildung am schlechtesten kreieren kann, ist die Fähigkeit und das Bedürfnis danach, weiter zu lernen."

LINDEMANS "The Meaning of Adult Education“ (1926), YEAXLEES „Lifelong Education“ (1929): Beide Autoren beschreiben „education“ als einen fortlaufenden Aspekt des alltäglichen Lebens: „[...] The whole of life is learning, therefore education can have no endings [...]" (LINDEMAN, 1929). Oder, mit YEAXLEES Worten ausgedrückt: „[...] Life, to be vivid, strong, and creative, demands constant reflection upon experiences [...]." (1929: 28)

YEAXLEE widmete sich vor allem dem Konzept des informellen Lernens, welches auch Menschen ohne spezielle Schulbildung anspricht. „[...] They (elementary and informal adult education) meet him where he is, and do not demand that he shall take a long journey, or make a violent and unnatural effort, to reach them. [...] But also they are the only ground wherein a large number of people will ever find themselves at home at all [...]" (1929: 155)

YEAXLEE sprach sich bereits zu seiner Zeit für die Idee, den Lernenden „dort abzuholen, wo er steht" aus, welches die nächste vorzustellende Methode des informellen Lernens ebenfalls beachtet.

\subsection{Methoden des informellen Lernens}

\subsubsection{Das „,show and tell“ Konzept}

Vor allem in Amerika ist in verschiedenen Unterrichtsformen ein Konzept bekannt und prominent, dass als 'show and tell' bezeichnet wird. Gemeinhin wird dabei ein realer Gegenstand 'präsentiert' (=show) und dazu 'etwas erzählt' (=tell), um damit verschiedene Wahrnehmungsebenen zu bedienen. Die Prominenz dieser Methode findet sich auch medial gespiegelt, beispielsweise in der beliebten

46 Leadbeater (2000), sinngemäß aus dem Englischen übersetzt 
Zeichentrickserie 'The Simpsons': In dieser Episode ${ }^{47}$ sollen die Schüler der Springfield Grundschule das 'show and tell' Konzept ausprobieren. Dem männlichen Kinderprotagonisten Bart Simpson ist das Konzept jedoch zunächst nicht bekannt, weswegen er statt eines Gegenstandes seinen Hund mitbringt, der sich nicht wie ein Gegenstand verhält, ausbricht, den Rektor verletzt und zu allerlei Problemen führt. Die Komik dieser Szene liegt nicht nur in den amüsanten Angelegenheiten, die der Hund durch seinen Ausbruch katalysiert, sondern vor allem auch darin, dass diese Methode in Amerika so populär ist und es nahezu undenkbar ist, dass ein Kind diese Methode nicht kennt und sie entsprechend missversteht.

Nicht nur in der Medienwelt finden sich 'show and tell' Beispiele. Auch in den 80 bis 90er Jahren des 20. Jahrhunderts gab es wissenschaftliche Studien dazu (CUsworTH 1995). Gemäß dem Abstract des Artikels sollte mit der Studie die tatsächliche Rolle von 'show and tell' oder auch 'Newstime' in den frühen Schulerfahrungen von (Kindergarten-) Kindern erörtert werden, wozu drei Fallstudien, eine Befragung von 392 Lehrern von Kindergarten bis K-2 Klassen in New South Wales und eine ethnographische Studie aus der Perspektive der Lehrer, Schüler und einer kleiner Gruppe von Eltern von Kindergartenkindern, herangezogen wurden.

Als Ergebnis hält CuswORTH fest, dass Disparitäten zwischen den pädagogischen Intentionen, wie sie in der Forschungsliteratur zu finden sind, den Absichten der Lehrer und der Umsetzung in den Klassen auftreten. Unter anderem verweist sie dabei darauf, dass Lehrer dabei eher die Entwicklung von Fähigkeiten im Sinn haben, als die Absicht Gelegenheiten für das Mitteilen mündlicher Narrative bereit zu stellen.

Den Nutzen von dieser Methode fasst CuswORTH wie folgt zusammen:

provide an opportunity for children to share a personal experience or favourite belonging from home in front of a large group; build a bridge between home and school experiences, between home and school knowledge; encourage the development of oral narrative which plays a crucial role in our thinking and language develpment (Bruner, 1986, Hardy and Rosen, 1984); and develop children's oracy as a precusor to literacy development (Christie, 1986, Fox, 1983, 1988, 1994, Collins and Michaels, 1986, Michaels, 1985a and b) (CUSWORTH 1995: Abstract:4)

47 Folge 19 Staffel 5, Erstausstrahlungsdatum laut imdb.com 17. Juni 1995, Produktionscode 1F18, http://www.imdb.com/title/tt0777150/; http://www.snpp.com/episodes/1F18.html, 
Es findet sich 1980 eine weitere Studie zum Thema 'show and tell' die kurz angeführt werden soll:

In der vorgestellten Studie, die zwei 'show and tell' Unterrichtsstunden im Abstand von 18 Tagen untersuchte, konnte gezeigt werden, dass durch eine Reduktion der Lehrerbeteiligung an den Gesprächen und ein weniger formelles Setting während 'show and tell' eine Steigerung der Partizipation der Kinder am Gespräch und eine Steigerung der Verantwortung auf Seiten der Kinder bezüglich der Organisation des Gesprächsflusses erbrachte. Auch konnte für die zweite Sitzung gezeigt werden, dass eine gesteigerte Qualität als auch Quantität hinsichtlich der Kommunikation der Kinder während der „Sharing Time“ Folge war (LAZARUS und HOMER 1980)

Eine gesteigerte Fähigkeit und intrinsische Motivation zur Partizipation am Gespräch und generellen Lernprozess ist auch für die in dieser Arbeit benannte Zielgruppe der gealterten Migranten erstrebenswert. Um dies zu erreichen, wird nach einem kurzen Exkurs zu 'sprachlichen Zeichen' das Konzept eines 'Infokoffers' vorgestellt um eine erfolgreiche Methode zu präsentieren, welche viele der bisher genannten Forderungen vereint.

\subsubsection{Exkurs: Was ist ein (sprachliches) Zeichen und wozu?}

Um wissenschaftliche Erkenntnisse über die Beschaffenheit von Sprachrezeptionsprozessen in der didaktischen Praxis nutzbar zu machen, ist es nötig, einen kurzen Seitenblick auf die Linguistik (Sprachwissenschaft) und (ihr Teilgebiet) die Semiotik zu werfen. Bei der Semiotik handelt es sich um die Wissenschaft, welche sich grundlegend mit Zeichensystemen aller Art beschäftigt. Dabei untersucht sie sowohl sprachliche als auch außersprachliche Zeichen. Um (sprachliche) Zeichen, beispielsweise in der interkulturellen Didaktik, gezielt, reflektiert und nachhaltig einsetzen zu können, ist es nützlich, sich beispielsweise die prominenten und grundlegenden Studien des Linguisten KARL BÜHLER, des Semiotikers CHARLES SANDERS PEIRCE und des Linguisten FERDINAND DE SAUSSURE in ihren Essenzen zu betrachten. Dies begründet sich vor allem darin, dass eine Theorie sprachlicher und nichtsprachlicher Zeichen ein grundlegendes Fundament der Erkenntnis- und Wissenschaftstheorien darstellt und für diese Arbeit als Herleitung sinnvoll ist. 


\section{Karl Bühler - das Organon-Modell $\underline{48}$}

Ausgehend vom aristotelischen ${ }^{49}$ Verständnis der Dreiecksbeziehung des Zeichens mit dem gesprochenen Wort, dem Gegenstand über welchen gesprochen wird und der Vorstellung, die damit in der Seele verbunden ist, entwickelte BüHLER zu Beginn des 20. Jahrhunderts sein sogenanntes OrganonModell. Der Begriff des Organon geht zurück auf PlATONS Kratylos. In diesem Werk bezeichnet SOKRATES die Sprache als ein geformtes Werkzeug (nicht nur Instrument und Hilfsmittel), durch welches eine Person einer anderen Person etwas über einen Sachverhalt oder Gegenstände mitteilt. BÜHLER stellt die Beziehung zwischen Sprecher und Empfänger über das Medium des Zeichens (im Zentrum) unter Einbeziehung des Kommunikationsinhalts wie folgt in seiner bekannten Grafik dar:

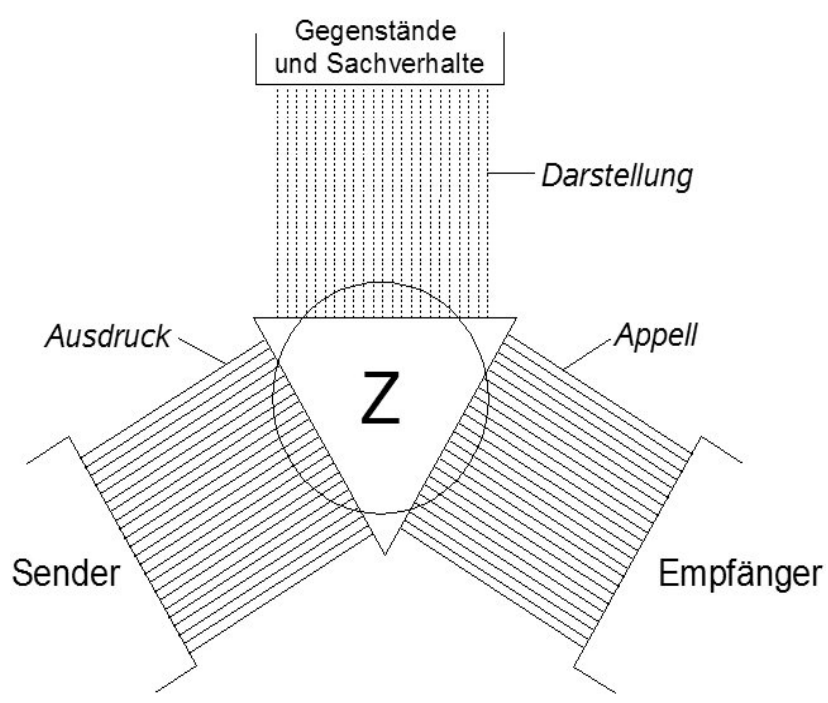

Abb. 3. 'Bühler-Organon-Modell' (vgl. Bühler 1978:24 ff.)

Der Sender bringt somit zum Ausdruck, was er dem Empfänger über Gegenstände und Sachverhalte mitteilen will und bedient sich 'des' Zeichens, als Medium. Der Kreis steht in dieser Darstellung stellvertretend für das

48 Vgl. KARL BÜHLER: Sprachtheorie. Die Darstellungsfunktion der Sprache. Frankfurt/Berlin/Wien 1978 , S. $24 \mathrm{ff}$.

49 "Die gesprochenen Worte sind die Zeichen von Vorstellungen in der Seele und die geschriebenen Worte sind die Zeichen von gesprochenen Worten. So wie nun die Schriftzeichen nicht bei allen Menschen dieselben sind, so sind auch die Worte nicht bei allen Menschen dieselben; aber die Vorstellungen in der Rede, deren unmittelbare Zeichen die Worte sind, sind bei allen Menschen dieselben und eben so sind die Gegenstände überall dieselben, von welchen diese Vorstellungen die Abbilder sind." ARISTOTELES, Peri hermeneias, Erstes Kapitel. 
'Schallphänomen' eines sprachlichen Zeichens, also für die lautliche Äußerung. Das mit ' $Z$ ' bezeichnete Dreieck stellt das sprachliche Zeichen mit seinen Funktionen (Darstellung, Ausdruck, Appell) dar. Zu erkennen ist, dass der Kreis an einigen Stellen über das Dreieck hinaus ragt und umgekehrt das Dreieieck über den Kreis hinauszielt. Ersteres Phänomen fasst Bühler unter dem Terminus der 'semiotischen' oder auch der 'abstrakten Relevanz' zusammen, das in nuce aussagt, dass unwichtige Informationen (zum Beispiel die Höhe eines Straßenschildes für das Verständnis dessen was es abbildet) ausgeblendet werden können. Interessanter für den Kontext dieser Arbeit ist jedoch das Phänomen der 'apperzeptiven Ergänzung', dargestellt durch die Schnittstellen, an denen das Dreieck über den Kreis hinaus ragt und damit auf die Defizite des materiellen Zeichenträgers ' $Z$ ' hinweist. Gemeinhin wird als Beispiel angeführt, dass durch laute Umgebung, der Empfänger den Sender nicht gänzlich verstehen kann, die Schalläußerungen also nicht vollständig vom Sender zum Empfänger übertragen werden. Der Empfänger versteht jedoch den Sender meist dennoch anhand der apperzeptiven Ergänzung, die sein Gehirn entsprechend vornimmt: das gemeinsame Inventar sprachlicher Zeichen, Erfahrungen, Kontexte und mögliche Äußerungen etc. werden blitzschnell abgeglichen und führen so zu einer 'gelungenen' Kommunikation. Alternative zur Störung durch Lautstärke, Artikulationsungenauigkeit, Umgebungslärm etc. kann auch eine dezente Abweichung im gemeinsamen Zeicheninventar genannt werden (z.B. einzelne Wörter sind dem Empfänger nicht bekannt). Kontextuell lassen sich diese jedoch meist erschließen, wenn der Vorrat an gemeinsamen Zeichen groß genug ist. Eine funktioniernde 'apperzeptive Ergänzung' erfordert somit eine Referenz des Senders und Empfängers auf dieselbe natürliche Sprache, eine gemeinsame (kulturelle) Lebenswelt und vor allem ein (möglichst) identisches Zeicheninventar. Doch auch eine gemeinsame Lebenswelt und ein gemeinsamer Zeichenvorrat können zu kommunikativen oder rezeptiven Missverständnissen führen. Der Strukturalist FERDINAND DE SAUSSURE lieferte früh einen Ansatz, der dies anschaulich erklärt. 


\section{Signifié und Signifian nach FERDINAND DE SAUSSURE}

Ende des 19. Jahrhunderts postulierte der Franzose FERDINAND DE SAUSSURE mit seinem Werk 'Cours de linguingistique générale' etwas, das sich jeweils auf alle natürlichen Einzelsprachen übertragen lässt: Eine der Grundeigenschaften eines sprachlichen Zeichens ist seine Bedeutung, die sich zusammensetzt aus der Ausdrucks- und der Inhaltsseite. Die Ausdrucksseite, ist damit sehr plastisch zu verstehen. Das Saussure'sche Beispiel ist gemeinhin die lautliche Äußerung 'Baum', die er als Signifiant bezeichnet. Äußert ein Sprecher (oder Schreiber) somit das 'Wort' Baum, entsteht automatisch im Kopf des Rezepienten ein Bild eines Baumes. Bei einem anderen Rezepienten entsteht bei der Äußerung des Signifiants 'Baum' auch sofort das Bild eines Baumes in dessen Vorstellung, es unterscheidet sich jedoch vom Bild des ersten Rezepienten, auch wenn es Ähnlichkeiten aufweist. Der eine Rezepient mag an eine Tanne denken, der nächste an eine Buche - bei beiden handelt es sich um einen Baum. Die Vorstellung (Inhaltsseite) eines Zeichens bezeichnet DE SAUSSURE als Signifié. Damit lässt sich schon andeuten, dass der Zusammenhang zwischen dem Bezeichneten (dem Baum in der Imagination) und dem Bezeichnenden (dem Wort Baum) untrennbar ist. Dennoch ist eine weitere Eigenschaft von sprachlichen Zeichen ihre Arbitrarität. Das mit dem Wort 'Baum' Bezeichnete könnte genauso gut Muab genannt werden, sofern innerhalb der Sprachgemeinschaft tradiert wird, dass dieses Signifiant das Signifié eines 'Baumes' bezeichnet. Die Thematik der Arbitrarität findet sich jedoch auch auf der Ebene der nonverbalen Zeichen, wie sie von CHARLES PEIRCE prominent beschrieben wurden.

\section{Ikon, Symbol und Index nach CHARLES S. PEIRCE ${ }^{51}$}

Da sich die Zeicheninventare natürlicher Sprachen unterscheiden, wird beispielsweise in spezifischen internationalen Kontexten auch auf nonverbale Zeichen zurückgegriffen, da einige von innen weniger missverständlich sind, als andere - obwohl generell alle Zeichen arbiträr sind. Der Semiotiker CHARLES

50 Vgl. Ferdinand de Saussure: Grundfragen der allgemeinen Sprachwissenschaft. Hrgs.v. Charles Bally. Berlin/New York 2001.

51 Vgl. Albert Busch und Oliver Stenschke: Germanistische Linguistik. Tübingen 2007, S. $19 f f$. 'Zeichentypen'. 
PEIRCE hat diese Zeichen in drei Kategorien aufgeteilt, die im Folgenden erläutert werden.

Zunächst ist das 'Ikon' zu nennen, dessen Haupteigenschaft ist, dass es sich um eine Abbildung eines Referenzträgers, der in der 'Realität' ähnlich aussieht. Nachvollziehbar wird ein Ikon am Beispiel der Piktogramme von beispielsweise dem Zeichen für ein Rauchverbot, das gemeinhin durch eine durchgestrichene Zigarette dargestellt wird. Die Zigarette wird eindeutig mit dem außersprachlichen Referenzträger in der Welt identifiziert, wenngleich die Aussage auf dem Schild bereits eine Mischform mit der dritten Kategorie (Index) darstellen könnte.

Neben dem Index, der im Anschluss erläutert wird, und dem Ikon, führte PEIRCE noch die Kategorie des Symbols ein. Ein 'Symbol' ist das wohl willkürlichste Zeichen, da es genau wie die Ausdrucksseite eines sprachlichen Zeichen gänzlich frei von Bedeutung ist. So wie der Wortklang 'Baum' dem biologischen Baum nicht ähnelt, ähneln ein abgespreizter Zeige- und Mittelfinger dem Zustand für 'Frieden' auch in keiner Weise, und sind somit rein kulturell belegt und geprägt. Auch hier können interkulturell Missverständnisse auftreten. Wird das Peace-Zeichen (wie eben beschrieben) beispielsweise mit der Handaußenseite einem Briten gezeigt, fühlt dieser sich kulturgeschichtlich möglicherweise beleidigt. Ein Deutscher hingegen könnte es als Siegeszeichen lesen, wie es vom ehemaligen Bundeskanzler Gerhard Schröder oft benutzt wurde. Kultursensibler Umgang ist also auch im Kontext der nonverbalen Zeichen gefordert. Noch deutlicher wird dies am Beispiel der Indices.

Neben dem Ikon und dem Symbol spricht PEIRCE von der Kategorie der Indices. Ein 'Index' stellt in Form eines Zeichens die Folge von einem Geschehen dar. Eine nasse Straße lässt üblicherweise auf vorherigen Regen schließen und steht damit als Zeichen für das vorangegangene Ereignis. Ob es jedoch wirklich geregnet hat, oder beispielsweise eine am Straßenrand befindlicher Rasenbefeuchter die Straße mit befeuchtet hat, leitet der Rezepient von seinem Weltwissen, Kontextwissen und aus seinen Erfahrungen ab, die interindividuell und interkulturell variieren. Indices müssen somit auch immer kultursensibel eingesetzt und konstruiert werden, da sonst ebenso wie bei sprachlichen Zeichen Missverständnisse entstehen können.

Ein prominentes Beispiel ist eine Anekdote aus der Linguistik, deren Quelle 
z.Z.d.V. nicht recherchierbar war: In Folge eines Krieges wurden über einem arabischsprachigen Land Versorgungspakete mit Babynahrung aus Flugzeugen abgeworfen. Um sicherzustellen, dass die Bewohner des Landes verstehen, was der Inhalt der Pakete war, entschieden sich die Paketersteller für Piktogramme, die ein trauriges Babygesicht, ein Zeichen für die Versorgung mit dem Inhalt des Pakets und dann als Folge ein lachendes Babygesicht enthielten. In der westlichen Welt, deren Leseverhalten von links nach rechts verläuft, auch ein gänzlich logischer Index. In der arabischen Welt verläuft die Leserichtung jedoch umgekehrt, was zu großen Ängsten und Unsicherheiten bei der Bevölkerung führte, da sie fürchteten ihren Kindern mit der Nahrung zu schaden. So wurden die meisten Pakete vernichtet. Dieses beispiel macht deutlich, dass auch beim Einsatz von nichtsprachlichen Zeichen kultursensibler Umgang gefordert ist.

\section{Zusammenfassung des Exkurses}

Trotz der angeführten Schwierigkeiten, die sich durch die Nutzung von Sprache bereits innerhalb einer Sprachgemeinschaft ergeben, bietet der Rückgriff auf nonverbale Zeichen, wie Ikone, Indices oder Symbole gerade im didaktischen, interkulturellen Kontext enorme Möglichkeiten, das Lernen und vor allem das Verstehen von Sachverhalten zu erleichtern. Menschen mit Zuwanderungsgeschichte, die kein oder nur wenig deutsch sprechen, haben nachvollziehbarer Weise größere Schwierigkeiten hinsichtlich der apperzeptiven Ergänzung, wenn ihnen Sachverhalte in einer Sprache vermittelt werden sollen, die sie

- nicht oder nur schlecht verstehen,

- deren kulturelle Mechanismen sie aufgrund dieser Sprachbarriere nicht oder kaum verstehen können (Stichwort: Arbitrarität von Worten),

- Sachverhalte in ihrer Ursprungskultur gar nicht existieren, entsprechend keine Wörter dafür vorhanden sind, die 'einfach' übersetzt werden könnten ${ }^{52}$.

52 Beispiel: Das Gegenwartsdeutsche kennt kein natürliches Wort mehr für den Zustand des 'nicht mehr durstig seins', der durch ein Kunstwort 'sitt' bezeichnet wird. Herrscht nun im Ursprungsland der Menschen mit Zuwanderungsgeschichte kein derartiges soziales Versorgungssystem wie beispielsweise in Deutschland, und es gibt dafür in der Ursprungssprache keine Wörter, kein kulturelles Verständnis dafür, sieht sich der Didaktier mit der Frage konfrontiert: Wie kommuniziere ich z.B. die Thematik der Versorgungsansprüche Menschen, die meine Sprache kaum bis gar nicht verstehen und deren Enkulturation und Sozialisation eine apperzeptive Ergänzung erschwert? 
Dies sind nur einige Beispiele für Schwierigkeiten, die durch Sprache auftreten können. Ein sprachreduziertes Konzept zur Vermittlung von Inhalten, dass entsprechend die Belastung durch Verständnis- und Verständigungsprobleme verringert, könnte als Kompensationswerkzeug dienlich sein. Um Verständigungsprobleme zu umgehen, entwickeln Menschen mit Zuwanderungsgeschichte Strategien, wie sie unangenehme Situationen bei kulturellen Differenzen (im Türkischen gibt es beispielsweise keine äquivalente Unterscheidung zwischen den Anredepronomina 'Sie' (singular, höflich) und 'Du'), können - wie Michael Weinhold ${ }^{53}$ postuliert:

Die Wahl des „richtigen“ Anredepronomen stellt besonders für Ausländer eine große Herausforderung dar, sind wir uns als Muttersprachler doch selbst manchmal nicht sicher, wann ein $D u$ oder doch das Sie angebracht ist." [...] „Desweiteren beobachtete die Beamtin, dass viele ihrer Kollegen „Randgruppen“ wie Alkoholiker, Obdachlose oder Ausländer duzten, sich im Gegenzug aber angegriffen fühlten, wenn diese das $D u$ erwidern. Erst dann wurde innen bewusst, dass ihre Sprechhaltung als beleidigend empfunden werden könnte und sie kehrten zum Sie zurück (Hadwiger 2001:20).“ [...]

Die angeführten Beispiele zeigen deutlich, dass es in der heutigen pluralistischen Gesellschaft nahezu unmöglich ist, konkrete Normen für den Gebrauch der Anredepronomina aufzustellen. Entsprechend verunsichernd können bestimmte Situationen für die Sprecher sein und entsprechend viele Faktoren sind bei der Wahl der Anredeform zur beachten: Alter, soziale Stellung, Kleidung, Umgebung usw. Im Zweifelsfall hilft die relativ unproblematische Lösung, das Anredepronomen komplett zu umgehen. Die Frage „Könntest Du / Könnten Sie mir bitte die Butter geben?“ lässt sich geschickt mit einer indirekten Formulierung wie „Könnte ich bitte die Butter haben?" umgehen. Mit zunehmender Dauer des Gesprächs wird es aber nicht nur immer schwieriger, diese Strategie beizubehalten, es wird auch die Unsicherheit des Sprechers über seine Beziehung zu seinem Gesprächspartner immer deutlicher, was die Kommunikation wiederum wesentlich beeinträchtigt (Hadwiger 2001:21). [...]

Wie bereits [...] dargestellt, kann eine falsche Verwendung der Anredepronomina auch als Beleidigung aufgefasst werden. Dies muss jedoch nicht immer juristische Folgen haben. Die Verletzung der Höflichkeitsregeln kann aber auch zu Unstimmigkeiten zwischen den Gesprächspartnern führen und zwischenmenschliche Beziehungen beeinträchtigen. Dies gilt nicht nur für die unangemessene Verwendung von $D u$. Auch ein Sie gegenüber einer eigentlich vertrauten Person kann als Zeichen der Geringschätzung der Beziehung interpretiert werden. (Weinhold 2010: 5f)

Obige Zitate zeigen beispielhaft wie Menschen mit Zuwanderungsgeschichte versuchen, Strategien zu entwickeln um sich der Belastung der fremden Sprache zu entziehen. Eine weitere Strategie kann sein, sich in die eigenen 'Herde' (Familie, Dunstkreis) zurück zu ziehen und den Kontakt mit Menschen 'der anderen Sprachgemeinschaft' so weit als möglich zu vermeiden. Dabei handelt es sich sowohl um ein interkulturelles Phänomen, als

53 Michael Weinhold: Spezialprobleme des Übersetzens SP-DE/DE-SP; Das Anredesystem des Deutschen. 2010 ; http://www.carstensinner.de/Lehre/spanisch/dossiers/Duzen_und_Siezen_im_Deutschen.pdf, 04.01.2011 
auch um ein intrakulturelles Problem. Insbesondere für die türkische Sprache lässt sich ableiten: Alte Migranten sprechen oftmals die 'Jugendsprache' der Gegenwartsgeneration nicht, verstehen jedoch aufgrund ihrer langen Abwesenheit aus dem Ursprungsland die dortige Gegenwartssprache auch nicht mehr vollständig, da sie nach ihrer Anwerbung als junge Menschen in Deutschland verweilten. Dennoch tritt das Phänomen auf, dass alte türkische ehemalige Gastarbeiter türkisches Fernsehen rezipieren, ohne die Sprache gänzlich zu verstehen. Über die Bilder und die emotionale Identifizierung mit dem Ursprungland findet jedoch eine Identitätsbestätigung statt, die eine Form von Stressreduktion darstellt. Eine andere Form der Stressreduktionsstrategie könnte auch sein, das Problem der Sprachbarriere zu explizieren. Prominente Beispiele aus der Medienlandschaft sind in diesem Kontext Comedians wie Kaya Yanar, Django Asyl, Bülent Celan oder Serdar Somuncu. Sie bedienen sich der Selbststereotypisierung nicht nur auf sprachlicher Ebene als Marketingmittel, sondern können anderen als Vorbild zur Konfliktvermeidung dienen. Öffentlich reflektiert wird die Selbststereotypisierung beispielsweise auch bei dem türkischstämmigen Schriftsteller Feridun Zaimoglu ('Kanakspraak').

Der Erwerb der Zweitsprache führt vor allem im Erwachsenenalter zu einer erhöhten Belastung, insbesondere wenn es sich um extrinsische Motivation handelt:

Anders als bei Kindern ist der Spracherwerb bei Erwachsenen, wenn sie bereits längere Zeit in Deutschland leben, häufig bereits fossilisiert. Es zeigt sich eine Verbindung von z.T. ausgeprägter Kommunikationsfähigkeit und Sprechflüssigkeit einerseits und eingeschränktem Wortschatz und v. a. abweichenden morpho-syntaktischen Merkmalen andererseits, die die Verständigung auch wieder erschweren. Solche fossilisierten Lernersprachen lassen sich - wenn überhaupt - nur sehr schwer ändern. (AHRENHOLZ 2010: 27)

Das Erlernen von Zweitsprachen kann in verschiedenen Altersstrufen verschiedene Relevanz zeigen. Hierbei wird in der theoretischen Diskussion auch die Identitätsfindung im Rahmen der Persönlichkeitsentwicklung (antizpiertes Lernen) und gleichzeitig die fossilierten Lernersprachen hervorgehoben, welche ein Erlernen der Zweitsprache im Erwachsenenalter nahezu unmöglich machen können und damit andere, kultursensible, didaktische Methoden zur Informationsvermittlung und zum Katalysieren intrinsischer Motivation bei den (alten) Lernenden nötig machen. 


\subsubsection{Lernen über Bilder: der „Infokoffer“}

Nachdem im Exkurs über sprachliche Zeichen dargestellt wurde, dass Ikone, als Abbildungen des lebensweltlichen Realität die geringste Missverständnisquote haben, sollen diese zusammen mit einem historischen Beispiel als erste Argumente dienen, ein sprachreduziertes didaktisches Modell vorzustellen: den Infokoffer.

Im bekannten 'Höhlengleichnis'54 PLATONS, erfolgt durch Schatten eine Abbildung der Wirklichkeit an die Wand in die Höhle, in der Menschen leben und nur die Möglichkeit haben, ihre Welt anhand der Schattenbilder an der Wand zu interpretieren. Ohne jedoch die Ähnlichkeit zu den reelen Refernzobjekten zu kennen, wie das bei der 'show and tell'-Methode der Fall ist, liegt auf der Hand, dass der Wissenserwerb und Lerneffekt geringer ist, als wenn die Menschen die Höhle verlassen und dort die gesehenen Bilder mit der Wirklichkeit abgleichen:

Und, sprach ich, wenn inn einer mit Gewalt von dort durch den unwegsamen und steilen Aufgang schleppte und nicht losließe, bis er inn an das Licht der Sonne gebracht hätte, wird er nicht viel Schmerzen haben und sich gar ungern schleppen lassen? Und wenn er nun an das Licht kommt und die Augen voll Strahlen hat, wird er nicht das Geringste sehen können von dem, was ihm nun für das Wahre gegeben wird. (PLATON)

Obiges Zitat sagt weiterhin aus, dass extrinsische Motivation nicht immer der Weg ist, neue Lebenswelten und Sachverhalte zu lehren.

Mit (Ab-)Bildern an der Wand, lässt sich somit auch außersprachlich etwas lernen. Motivieren sich die Höhlenbewohner nun selbst, die Höhle zu verlassen und die Bilder, die sie an der Wand wahrgenommen haben, in der Realität zu suchen, abzuprüfen, zu verstehen und Neues zu lernen, ist in nuce erreicht, was ein Musterkoffer beispielsweise leisten kann: ein Abbild der Realität anbieten, um diese näher zu bringen und neugierig zu machen darauf, wie die 'echte' Welt aussieht und somit intrinsische Motivation herstellen. Die Idee eines Musterkoffers ist jedoch keine Erfindung des 21. Jahrhunderts, in dieser Art jedoch eine Weiterentwicklung.

${ }^{54}$ Vgl. Platon: Politeia. (Übersetzer: Friedrich Schleiermacher). Siebentes Buch, 106. b) Das Hinaufsteigen zum Licht und das Wiederherabkommen in die Höhle. 


\section{Musterkoffer}

Die Geschichte des Musterkoffers reicht bis ins Mittelalter zurück. Dies belegt beispielsweise ein Stück aus der orthopädischen Sammlung des Pathologen DR. KARL ANTON PORTELE: „Ein Unikum dieser Orthopädie-Sammlung ist sicher der Musterkoffer eines englischen Orthopädiemechanikers aus der Zeit um 1900. In einem eleganten, luxuriösen Köfferchen finden sich Prothesen und andere orthopädische Behelfe, die aus einer etwas skurrilen Puppenstube stammen könnten. Neben verschiedenen einfachen Krücken beinhaltet das Köfferchen natürlich das klassische Oberschenkelstelzbein, das berühmte Seeräuberbein für den armen Mann, und Holzprothesen mit beweglichem Vorfuß für die etwas betuchtere Kundschaft.“ (REGAL UND NANUT, 2005: 27)

Das Apothekenmuseum „Zum Goldenen Adler“ in Budapest zeigt eine Reiseapotheke aus dem Mittelalter: einen kleinen Koffer, in dem Glasgefäße mit Medikamenten transportiert wurden ${ }^{55}$. Ebenfalls im Mittelalter wurden bei Gefahr die Musterkoffer der Kaufleute vergraben, da diese zu wertvoll waren, und somit auch den 'Wert' der Gegenstände und Methode die der Koffer beinhaltet verdeutlicht (vgl. KULISCHER 1988). Nicht mehr im Mittelalter dann, im Jahre 1866 benutzte LEVI STRAUSS, der Vater der berühmten Blue Jeans, einen Musterkoffer als reisender Händler. „Das Geschäftsprinzip ist einfach - einfach genial: Was die Brüder an der Ostküste einkaufen, verkauft Großhändler Levi am Pazifik weiter. Ohne jemals selbst auf Goldsuche zu gehen, erschließt Levi Strauss für sein Unternehmen eine Goldgrube: Im Jahre 1866 besitzt die Firma bereits ein vierstöckiges Warenkontor in der Battery Street in San Francisco - gefüllt mit Kurzwaren und Bekleidung. Auch mit Hosen; die aber machen zu diesem Zeitpunkt nur einen kleinen Teil des Geschäfts aus. Die Musterkoffer in der Hand, die Kataloge im Rucksack, reisen die Handelsvertreter des Hauses Levi Strauss durch den Westen."(HÖRZ, 2002) 56

Der Musterkoffer taucht erneut Mitte des 20. Jahrhunderts auf. Dies zeigte eine Ausstellung zur Geschichte des Designs im Werkbundarchiv Berlin. „In den 50er und 60er Jahren zogen Werkbund-Jünger mit Musterkoffern durch die

\footnotetext{
55 http://www.miovista.de/europaweit/budapest/blog/budapest-entdecken/man-nehme56 mumienpluver-opium-und-eine-prise-lebensweisheit-28052008.html, Zugriff am 04.08.2009 
Klassenzimmer, um auch dort für guten Geschmack zu werben. "Der gut gedeckte Tisch" war ein Thema, andere Musterkoffer zeigten den idealen Schreibtisch oder beschäftigten sich mit Glas oder Porzellan. Das waren skurrile Erziehungsversuche, doch die Koffer wurden im Dienst einer neu gewonnenen Freiheit gepackt."(STOCK, 2007) ${ }^{57}$

Auch in jüngerer Zeit finden sich vielfältige Beispiele für den Gebrauch eines Koffers als Präsentationsinstrument. Die Medienkommission des Bundesvereins zur Prävention von sexuellem Missbrauch stellt den von einer Beratungsstelle herausgegebenen Koffer „Nachtlicht“ mit Informationsmaterial zum Problem der Kindesmisshandlung und des Kindesmissbrauchs vor. Dieser Koffer soll Kinder und Erwachsene ansprechen, auf das Thema aufmerksam machen, dafür sensibilisieren und Bewältigungsmöglichkeiten aufzeigen. Er beinhaltet einen Videofilm, verschiedene Arbeitshefte und Broschüren und Hinweise zu regionalen Hilfsangeboten. (MEDIENKOMMISSION DES BUNDESVEREINS

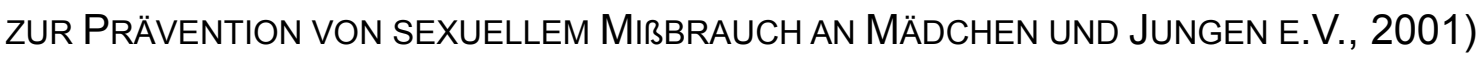

Die Münchener Koordinierungsstelle für gleichgeschlechtliche Lebensweisen brachte in Kooperation mit dem Stadtjugendamt einen „didaktischen Koffer zum Thema Lebensformen“ für Kinder im Alter von 9-12 Jahren heraus. Dieser Koffer kann in Schulen, Horten oder sonstigen angeleiteten Kindergruppen verwendet werden, um die Kinder mithilfe der sechs enthaltenen Spiele an verschiedene Lebensentwürfe und Familienformen heranzuführen. Dabei sollen Respekt, Toleranz und Selbstbestimmung gelernt werden. „Die pädagogische Arbeit mit dem Koffer dient der Aufklärung und Information über Lebensformen sowie der Prävention von Diskriminierung und Gewalt.“ (UNTERFORSTHUBER, O.D.)

Koffer zur Präsentation von Inhalten sind natürlich auch weiterhin in gewerblichen Bereichen von Bedeutung. Im Beruf des Bestatters beispielsweise werden Hinterbliebene zu Hause mithilfe von Musterkoffern über die verschiedenen Angebote informiert. ${ }^{58}$

57 http://www.dradio.de/dkultur/sendungen/fazit/641411/ Zugriff am 04.08.2009

58 http://www.bestattungen-ernst.de/cid/index.html?dlr-Page_uni_008/index.html, Zugriff am 15.09.2009 


\section{Die Kurzbeschreibung zur türkischen Inforeihe}

\section{Zur Beschreibung des Infokoffers findet sich auf der entsprechenden}

Webseite sehr trefflich:

Die Inforeihe entstand in Anlehnung an das niederländische Vorbild „Ouder worden in Nederland" (des NIZW) und wurde von einem interdisziplinären und interkulturellen Expertenteam für die Situation in Deutschland übertragen. Ziel der Inforeihe ist es, ältere Migranten/innen (hier ältere Türken/innen) mit den vor Ort vorhandenen Angeboten der Altenhilfe vertraut zu machen und umgekehrt die Einrichtungen der Altenhilfe mit den älteren Migranten/innen, ihrer Lebenssituation und ihren Bedürfnissen an eine angemessene Betreuung bekannt zu machen. Grundlage der Inforeihe ist der Einsatz eines Medienkoffers. Die Materialien (Video, Foto, Folien usw.) können bundesweit genutzt werden.

Das Konzept beinhaltet eine zweisprachige Seminardurchführung. Dazu bilden zwei Fachkräfte, eine einheimische und eine mit Migrationshintergrund aus den Feldern Altenhilfe und Migrationssozialarbeit ein Team und kooperieren partnerschaftlich bei allen Schritten; angefangen bei der Planung, der Kontaktaufnahme, der Werbung bis zur Durchführung und Auswertung. In der Vorbereitungsphase initiieren sie eine örtliche Arbeitsgruppe mit Multiplikatoren/innen aus Migranten-Selbstorganisationen und aus der Altenhilfe, die die Durchführung der Informationsreihe in Abständen begleitet und in die auch die Wünsche und Bedürfnisse der älteren Migranten/innen nach Abschluss der Inforeihe zurückgemeldet werden.

Die 6 Treffen der Inforeihe sind so konzipiert, dass sie bausteinartig aufeinander aufbauen und die Teilnehmer einen Prozess durchlaufen, an dessen Ende die Migrantinnen und Migranten genügend Einblicke in die Leistungen und Einrichtungen der Altenhilfe gewonnen haben, um entscheiden zu können, ob sie diese bei Bedarf nutzen möchten.

Thematisch werden zuerst die emotional sehr belastenden Themen „Rückkehr in die Türkei oder Lebensmittelpunkt in Deutschland“ sowie „Hilfeleistungen durch die eigenen Kinder" aufgegriffen und bearbeitet. Ungeachtet dessen, ob die Kinder die Betreuung übernehmen oder sie zurückkehren werden, haben sie das Recht, die Angebote der Altenhilfe zu kennen und informiert zu sein. Damit wird eine Klärung dieser emotional belasteten Themen erreicht und die Grundlage für die Aufnahme neuer Informationen geschaffen.

Die einzelnen Hilfsangebote werden daraufhin bildhaft sukzessive vorgestellt. Danach findet eine Exkursion zu interessierenden, vor Ort vorhandenen und für die Teilnehmer erreichbaren Einrichtungen der Altenhilfe statt. Durch diese Besuche machen die Teilnehmer/innen Erfahrungen, die oft zu einer positiven Einstellungsveränderung gegenüber den Hilfsangeboten und den Einrichtungen führen. Umgekehrt erleben die Mitarbeiter/innen der Altenhilfeeinrichtungen, dass es einfacher ist als vorher angenommen, mit Migranten/innen in Kontakt zu kommen und dass es sehr befriedigend sein kann, zusammen zu arbeiten.

In einem letzten Schritt sollen die Teilnehmer die vorgestellten Angebote hinsichtlich ihrer Nutzbarkeit für sich selber beurteilen und Änderungswünsche bzw. neue Angebotswünsche benennen. Dies geht als Rückmeldung an die besuchten und in der Arbeitsgruppe vertretenen Einrichtungen, mit denen über die Möglichkeiten der Umsetzung verhandelt wird.

Damit kommt es zu einer schrittweisen Annäherung von Migranten/innen an die Altenhilfe und der Altenhilfe an die Migranten/innen. Dies fördert die Vernetzung vor Ort und trägt auch dazu bei, dass ältere Migranten/innen als Zielgruppe zukünftiger kommunaler Altenhilfe-Planung stärker wahrgenommen werden. ${ }^{59}$

\footnotetext{
${ }^{59} \mathrm{http}$ ://www.aelter-werden-in.de//index.php?option=com_content\&task=view\&id=12\&Itemid=46;
} letzter Zugriff 17.06.2011 


\section{Die Kurzbeschreibung zur türkischen Inforeihe-Materialien}

Zur Veranschaulichung der Materialien im Infokoffer folgt nun exemplarisch eine Darstellung der türkischen Inforeihe 'Älter werden in Deutschland'. Analog finden sich diese Materialien in der 'Informationsreihe für ältere Aussiedlerinnen und Aussiedler und ihre Ehepartner'.

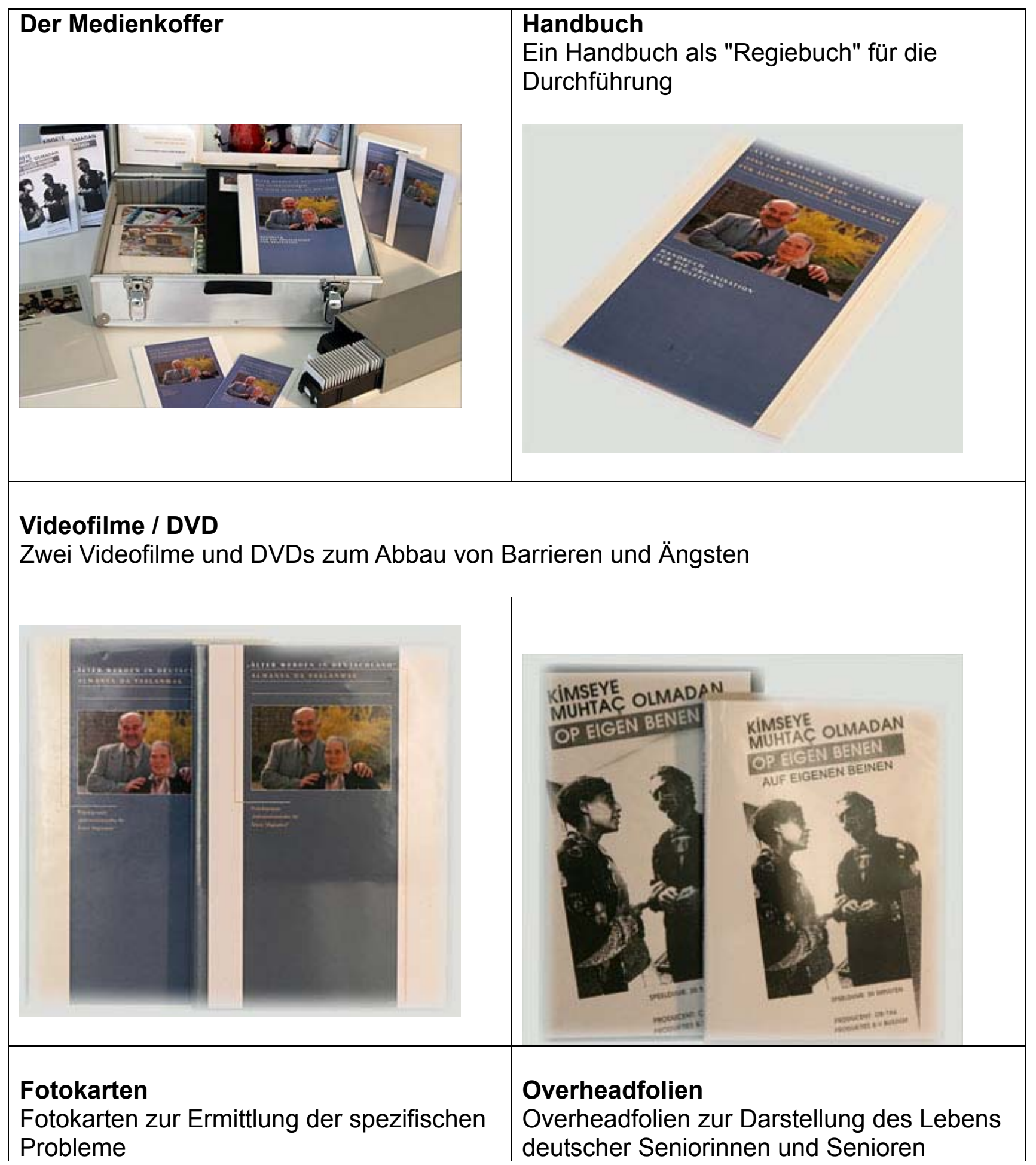




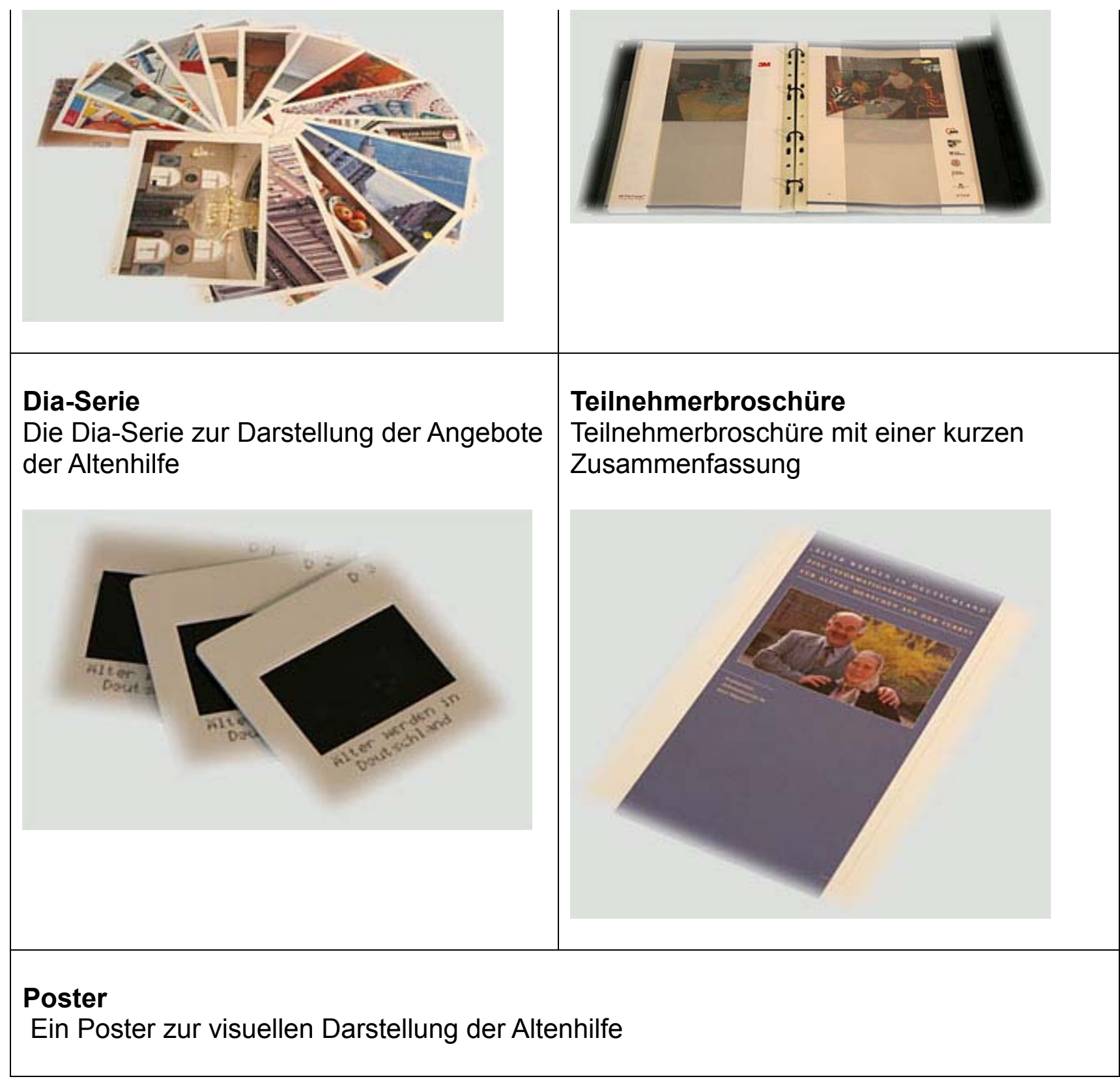

Bildquelle: http://www.aelter-werden-

in.de//index.php?option=com_content\&task=view\&id=38\&Itemid=169, letzter Zugriff: 17.06.2011

\subsection{Didaktik - wozu, wohin?}

Wie zu Beginn dieses Kapitels schon erwähnt, haben sich FLECHSIG und HALLER bewusst gegen eine Ein-Satz-Definition von Didaktik entschieden. Gerade deshalb soll an dieser Stelle nochmals der Versuch unternommen werden, einen Didaktikbegriff (nicht nur einen Lernenbegriff wie in den vorherigen Abschnitten) zu ergründen, auf dem im nächsten Kapitel die Neurodiadaktik aufbaut und damit die 
Schnittstelle zwischen Pädagogik und Neurowissenschaft bildet, welche einen der Grundpfeiler dieser Arbeit darstellt. KARL G. ZENKE definiert Didaktik wie folgt im Wörterbuch Pädagogik:

Didaktik (griech. didaskein, aktiv: lehren, unterrichten; passiv: lernen, belehrt werden; auch: sich aneignen, didaxis Lehre, Unterricht; engl. Didactics).

Didaktik ist im umfassenden Sinn der allgemeinen Didaktik die Wissenschaft des Lehrens und Lernens in allen pädagogischen Handlungsfeldern (z.B. Schule, Volkshochschule, Jugendarbeit, Universität) und im schulpädagogischen Sinn die Theorie des Unterrichts.

Didaktisches Denken und Fragen hat sich zum ersten Mal im antiken Griechenland bei den Sophisten, bei Sokrates und Platon artikuliert, als tief greifende weltanschauliche, wirtschaftliche und sozialeVeränderungsprozesse im 5. Jh. v. Chr. Bewusstes und geplantes Erziehen und Unterrichten notwendig machten. Dieses Denken und Handeln hat im Lehrplan der sog. Septem artes liberales (sieben freien Künste) institutionalisierte Formen angenommen und den Lehrplan des Abendlandes viele Jahrhunderte bestimmt. Als pädagogischer Begriff wird die Bezeichnung Didactica (Lehrkunst) zum ersten Mal 1613 in einem Bericht über pädagogische Reformvorschläge von W. Ratke gebraucht und dann 1657 in der >Didactica magna (große Lehrkunst) von J. A. Comenius als Titel einer Unterrichtslehre verwendet. Comenius ging es darum, »allen Menschen alles zu lehren«. Im 19. Jh. wird die Didaktik bei J.F. Herbart, O. Willmann und W. Rein wieder stärker auf den Unterricht bezogen und ihr Zusammenhang mit Pädagogik und Erziehung diskutiert.

(ZENKE 2000:152)

Didaktik wird somit verstanden als Wissenschaft des Lernens und Lehrens, die in allen pädagogischen Handlungsfeldern eingesetzt werden kann. HALLER und FLECHSIG gehen in diesem Zusammenhang näher auf der Pädagogikbegriff vor dem 19. Jahrhundert ein und erwähnen dabei unter anderem den 'Lehrer' COMENIUS, der jedem Menschen das Lernen ermöglichen wollte und damit im Grunde dem Geiste dieser Arbeit entspricht, die unter anderem einen Anreiz bietet, wie gerade benachteiligten Menschen - wie Migranten es aus verschiedenen z.B. sozio-ökonomischen und sozio-kulturellen Gründen sind eine gute Möglichkeit zu Lernen geboten werden kann:

Schon vor dem 19. Jahrhundert waren einzelne Pädagogen mit dem Anspruch auf den Plan getreten, eine besonders zweckmäßige Methode für das Lehren anbieten zu können. Besonders bekannt wurden als Autoren von "Universalmethoden" Jan Amos Komensky (Comenius) (1592-1670) und Johann Bernhard Basedow (1724-1790). Als erster Pädagoge jedoch, der eine wissenschaftlich-systematische Begründung der Unterrichtsmethode gab, ist Johann Friedrich Herbart (1776-1841) zu erwähnen, der das Lehrverfahren aus der systematischen Analyse des individuellen Lernprozesses ableitete.

(FLECHSIG UND HALLER 1975: 140)

Da jeder Mensch ein anderer Lerntyp ist, und gerade benachteiligte Gruppen wie die Menschen mit Zuwanderungsgeschichte eine besondere Situation brauchen, die kultursensiblen Raum zum Lernen schafft, wird an dieser Stelle auf FLECHSIG und HALLERS Überlegung zur spezifischen 'Lernsituation', die 
der jeweiligen Lebenssituation des Lerners angepasst sein muss, hingewiesen. Ein besonderes Augenmerk verdient auch der biografische Aspekt, den die Autoren benennen und entsprechend eine Klassifikation von 'Lernsituationen' entwickeln:

\begin{abstract}
gewisse Begründung finden:
- Lernen durch Tun,

- Lernen durch Simulation und Spiel,

- Lernen durch Erkunden und Forschen,

- Lernen durch Lehren,

- Lernen mit audiovisuellen Medien,

- Lernen durch Vortrag, Gespräch und Diskussion,

- Lernen mit gedruckten Texten.

(Flechsig und Haller: ebd.)
\end{abstract}

Indem wir unter "Lernsituationen" diejenigen Lebenssituationen verstehen, in denen Menschen primär lernen (obwohl sie gleichzeitig produzieren, sich unterhalten oder müßiggehen können), und indem wir von "Organisation" immer dann sprechen, wenn ein gewisses Maß an Absicht und Planung (im Sinne von Lernorganisation) vorliegt, ist es uns möglich, ein breites Spektrum von Lebenssituationen als organisierte Lernsituationen zu begreifen (vgl. auch Kapitel 2). Anknüpfend an unseren auf S. 202 f. erläuterten Medienbegriff können wir ferner annehmen, dass die Erfahrungen, die in solchen organisierten Lernsituationen gemacht werden, durch verschiedene Medien vermittelt sein können. Ausgehend von diesen Vorüberlegungen dürfte die folgende Klassifikation eine

Aus den zitierten Überlegungen entwickelten Flechsig und Haller einen erweiterten Lernbegriff, der untrennbar mit dem Didaktikbegriff verbunden ist:

Der sechste Aspekt des erweiterten Lernbegriffs [der dem Göttinger Katalog Didaktischer Modelle ebenso wie dieser Arbeit und dem Buch aus dem dieses Zitat stammt zugrunde liegt, I.Ö.] ergibt sich schon aus der Tatsache seiner Behandlung in internationalen Gremien und deren Orientierung an einer weltweiten, globalen Perspektive: Nationales und globales Lernen. Dem muß man gegenwärtig noch den Hinweis auf den Umstand hinzufügen, daß auch das Lernen auf lokaler und regionaler Ebene sowie in inter- und multikulturellen Kontexten für breite Bevölkerungskreise neue Bedeutung erhält, angefangen von Bürgerinitiativen und Gastarbeitern bis hin zu hohen Beamten internationaler Organisationen. Es kann künftig nicht mehr ausreichen, daß Probleme von Unterricht und Lernen in der Selbstgenügsamkeit nationaler Bezugsgruppen verhandelt werden. Die Gliederung der für das öffentliche Bildungswesen zuständigen Administrationen in nationale und subnationale Organe kann kein hinreichender Grund für eine Unterrichtswissenschaft mehr sein, ihre analytischen und praktischen Bemühungen allein im Rahmen nationaler Bezugsgruppen und Bezugssysteme zu behandeln. Dies gilt für Lehrpläne und Lehr-Lernformen ebenso wie für Lernbedingungen und Wechselbeziehungen von Bildungseinrichtungen und Kultursystem. Es gilt vielmehr, auf das gesamte Inventar der von der Gattung Mensch im Laufe der Geschichte entwickelten Erfahrungen, Deutungsmuster und Werkzeuge zurückzugreifen und einer selbstgenügsamen nationalen oder gar kleingruppenbezogenen Lernperspektive eine Absage zu erteilen, zunächst auf der konzeptionellen und - bei aller Anerkennung der damit verbundenen informationsmäßigen und technischen Schwierigkeiten auch auf der praktischen Ebene der Didaktik.(FLECHSIG und HALER 2003: 19f)

In diesem Kontext verweisen FLECHSIG und HALLER zudem auf den sogenannten 'Lernbericht an den CLUB OF ROME'. Hierbei geht der Lernbegriff über das konventionelle Vokuabular von Unterricht hinaus und nähert sich im weitesten Sinne bereits mehr dem generellen Thema Leben und damit natürlich auch Lernen und 


\section{Wissen an.}

Zusammenfassend sei auf eine Formulierung hingewiesen, die in dem bereits erwähnten "LERNBERICHT AN DEN CLUB OF ROME" zu finden ist, eine Formulierung, die den erweiterten Lernbegriff wie folgt faßt: "Den Begriff Lernen möchten wir in einem Sinn verstanden wissen, der über das konventionelle Vokabular von Erziehung (Unterricht), Ausbildung und Schulwesen hinausgeht, das heißt in seinem weitesten Sinn. Für uns bedeutet Lernen ein Sich-Annähern sowohl an das Wissen als auch an das Leben, bei dem der Nachdruck auf der menschlichen Initiative liegt. Es bedarf der Erwerbung und Anwendung neuer Methoden, neuer Fertigkeiten, neuer Verhaltensweisen und neuer Werte, um in einer sich verändernden 'Welt bestehen zu können. Lernen ist der Prozeß der Vorbereitung auf neue Situa-tionen (PECCEI, a.a.o., S. 28).(FLECHSIG und HALLER 2003: 20)

Ebenfalls berücksichtigt RUSTERMEYER 2007 den zuvor bei FLECHSIG und HALLER erwähnten biografischen Aspekt des Lernens. Sie benennt ebenfalls Lernerfahrungen, die das künftige Lernen prägen. In diesem Fall ist von der 'Erlernten Hilflosigkeit' die Rede. Eine Person wird dann erlernt hilflos, wenn sie die Selbstüberzeugung erlangt, etwas nicht zu können. Dazu kommt es durch zu viele negative Erfahrungen. Dadurch verliert er die Kontrolle und intrinsische Motivation kann gar nicht erst entstehen. Somit sind andere Lernbedingungen nötig:

Die Theorie der Erlernten Hilflosigkeit wurde von Seligman [...] entwickelt und zunächst in der Klinischen Psychologie, dann jedoch sehr rasch auch in der Pädagogischen Psychologie [...] intensiv erforscht. Im Zentrum der Theorier steht die Erwartung, Ereignisse, insbesondere negativer Art, nicht kontrollieren oder beeinflussen zu können. Die Konsequenzen der Erlernten Hilflosigkeil zeigen sich in vielen Bereichen [...] (RUSTEMEYER 2007: 19)

Als Gründe für die Entstehung erlernter Hilflosigkeit nennt RUSTERMEYER Aspekte, die speziell für Migranten auch zutreffen, die zu Integrationskursen verpflichtet werden, und unter dem Druck stehen, diese bestehen zu müssen:

Hilflosigkeit und Hoffnungslosigkeit entstehen vor allem dann, wenn für negative Ereignisse globale, internale und stabile Ursachen herangezogen werden. In Leistungssituationen betrifft das vor allem den Ursachenfaktor der mangelnden Fähigkeit. Werden Leistungsdefizite so erklärt, sinken die Erfolgserwartung und die Motivation und der Zusammenhang zwischen der eigenen Anstrengung und dem Resultat geht verloren [...] (RUSTEMEYER 2007: 21)

Es ist somit erstrebenswert den Erwartungsdruck nicht zu erhöhen und eine defizitfokussierte Haltung als 'Lehrender' einzunehmen. Es ist wichtig, die Selbstwirksamkeit der Lernenden zu bekräftigen und ihr Selbstbewusstsein zu stärken um intrinsische Motivation zusätzlich zu entfachen:

Die subjektiv wahrgenommene Selbstwirksamkeit wirkt sich auf die Motivation, das Lernverhalten und die angewendete Anstrengung aus. Menschen mit hoher Selbstwirksamkeit arbeiten länger und intensiver. Sie geben nicht so schnell auf, auch bei 
Lernzielen, die schwierig sind. Misserfolge werden nicht als Lernhemmung interpretiert, wenn die Person im Sinne einer hohen Selbstwirksamkeit annimmt, dass sie zu einer Verbesserung fähig ist. (RUSTEMEYER 2007: 26-27)

Ein weiteres pädagogisches Standardwerk kritisiert das vormals populäre 'Input-Output-Modell' und schafft damit einen weiteren Pfeiler für den Übergang zur Neurodidaktik. Es betont entsprechend FLECHSIG und HALLER (2003) die Notwendigkeit von Lernorganisation anstatt'althergebrachter' Didaktik:

Die Konsequenz: Didaktik funktioniert nicht nach dem "Input-Output-Modell "(Nürnberger Trichter, für alle wissenschaftlich objektiviert), sondern kann nur Anreizstrukturen für die je individuell Lernenden geben, die das lernende Subjekt dann im Sinne der Selbstorganisation weiterverarbeitet. Didaktik kann nur eine "Modellierung von Lernwelten" sein - so der Titel des Buches. Kösel zeigt dies sehr praktisch an der Integration einer Fülle von ganzheitlichen Lernkonzepten aus der humanistischen Psychologie und Pädagogik, ohne die Frage nach der Bedeutung der "Sache" im Bildungsprozeß aus den Augen zu verlieren. - Es bleibt abzuwarten, wie dieser Ansatz einer konsequent "subjektiven Didaktik" in der pädagogischen Diskussion rezipiert wird.

(GUJONS 1995: 250f)

Eine Selbstorganisation impliziert jedoch zugleich ein gewisses Maß an intrinsischer Motivation. Ein Verhalten ist dann intrinsisch motiviert,

wenn es nicht um seiner Konsequenzen und Ergebnisse willen ausgeführt wird und nur aufgrund der damit verbundenen positiven Gedanken und Gefühle für die Person verstärkend ist. Intrinsische Motivation kann somit definiert werden als Wunsch oder Absicht, eine bestimmte Handlung um ihrer selbst willen durchzuführen, weil die Handlung selbst als interessant, spannend, herausfordernd usw. erscheint (Schiefele und Köller 2006, S. 303). Im Falle einer intrinsisch motivierten Lernhandlung einer Schülerin oder eines Schülers spricht man von einer intrinsischen Lernmotivation. (RUSTEMEYER 2007: 29)

Intrinsische Motivation steht also in direktem Zusammenhang mit Selbstwirksamkeit. Inwiefern sich dies von Selbstkonditionierung unterscheidet, kann anhand der Erkenntnisse zur Neurobiologie des Lernens im nächsten Abschnitt geklärt werden.

\subsection{Neurobiologie des Lernens}

Der heutige Kenntnisstand über die neurobiologischen Grundlagen des Lernens basiert hauptsächtlich auf dem Prinzip der Konditionierung. Beim Lernen nutzen sowohl Tiere, als auch Menschen ihre Fähigkeit zur Assoziation von Sinneseindrücken und verbinden diese wiederum mit ihren bisherigen Lernerfahrungen. Dies geschieht durch die Neubildung oder Verstärkung von neuronalen Verknüpfungen (den Synapsen ) bei gleichzeitiger Reizaktivität in zwei Neuronen oder Neuronengruppen. Die zeitliche Kontingenz der Reize ist hierbei 
entscheidend. Bei oftmaliger Aktivierung derselben Synapse durch gleiche oder ähnliche Reize wird die dadurch repräsentierte Information dauerhaft im Gedächtnis gespeichert, bei längerer Nichtaktivierung der Verknüpfung wieder gelöscht. Beim Lernen sind daher viele kurze Wiederholungsphasen effektiver als wenige lange.

Unser Gedächtnissystem besteht aus mehreren verschiedenen Gehirnzentren, die durch ein weitverzweigtes neuronales Netz miteinander korrespondieren. Das Kurzzeitgedächtnis ist demnach in anderen Strukturen lokalisiert als das Langzeitgedächtnis. Durch Untersuchungen von Patienten mit Hirnschädigungen konnten wichtige Verarbeitungsprozesse des Lernvorgangs in verschiedenen Gehirnarealen lokalisiert werden. Die bedeutendste Rolle spielt dabei die Großhirnrinde (der Kortex), die den größten Teil des Gehirns ausmacht. Sie steuert elementare kognitive Vorgänge wie z.B. die Aufmerksamkeitszuwendung, ist aber auch für komplexere Formen des Lernens, Planens, Sprechens und schlussfolgernden Denkens zuständig. Der Hippocampus, eine am inneren Rand des Temporallappens liegende Hirnstruktur, ist an der kurzzeitigen Speicherung von neuen Informationen beteiligt und bietet diese dem Kortex wiederholt dar, um eine dauerhafte Speicherung zu erwirken.

Trotz der funktionalen Spezialisierung des Kortex ist bei der Informationsverarbeitung das ganze Gehirn beteiligt. Kognitive Aufgaben werden von verschiedenen, miteinander kommunizierenden neuronalen Systemen ausgeführt. Die Verarbeitung geschieht dabei durch ein Zusammenspiel von sogenannten „bottom-up-“ und "top-down"-Prozessen, wobei „bottom-up“ einen durch sensorische Reize initiierten kognitiven Vorgang bezeichnet und "top-down" die kognitiven Einflüsse auf die sensorische Wahrnehmung.

An dieser Stelle kann nun nochmals die Brücke zum vorherigen DidaktikAbschnitt geschlagen werden:

Aus der Neurobiologie des Lernens werden von Hirnforschern in aktuellen wissenschaftlichen Werken zunehmend didaktische Konzepte und Vorschläge (ganz im Sinne des antizipierenden Lernens) formuliert, die über genaue Funktionsweisen und biologische Zusammenhänge in der individuellen Verarbeitung heraus interessante Ansätze liefern, wie z.B. BRAND und 


\title{
MARKOWITSCH (2009) zur Neurodidaktik ${ }^{60}$ schreiben:
}

Vermutlich liegt in diesen multiplen Verbindungen des orbitofrontalen Cortex mit verschiedenen limbischen Strukturen (z.B. Amygdala, die für die Verarbeitung von Emotionen beideutsam ist, vgl. oben), aber auch mit anderen Teilen des Stirnhirns, der Schlüssel für das Verständnis der sehr integrativen Leistungen dieser Region. Hier werden persönliche Erinnerungen mit der entsprechenden emotionalen Bedeutung und einhergehendem emotionalem Erleben (während des Erinnerns) assoziiert, sodass es zu einer ganzheitlichen, emotional gefärbten und Ich-bezogenen Erinnerung distinkter Ereignisse kommen kann (zur Bedeutung des orbitofrontalen Cortex siehe Brand/Markowitsch 2006a). (BRAND und MARKOWITSCH 2009: 78ff)

\section{Gleichermaßen werden an dieser Stelle Verknüpfungen von Gedächtnis,}

Lernen, Prägung und Beziehung aus neurobiologischer Sicht mitgeliefert. Ebenso wie die innere Dynamik eines Individuums beleuchtet wird, die generell nicht (leicht) biologisch zu fassen oder messen ist, können jedoch über die hirnbiologischen Prozesse Ableitungen für die Didaktik - nach HÜTHER (2009) getroffen werden:

\begin{abstract}
Verankert werden diese Metakompetenzen in Form komplexer Verschaltungsmuster in einer Hirnregion, die sich im vorderen Großhirnbereich befindet: im Stirnlappen, dem präfrontalen Kortex. Die in anderen Hirnregionen gespeicherte Gedächtnisinhalte werden in diesen Netzwerken des präfrontalen Kortex zu einem Gesamtbild zusammengefügt und mit den in tiefer liegenden subkortikalen Hirnbereichen generierten Signalmustern verglichen. Die so erhaltenen Informationen werden für alle bewussten Entscheidungsprozesse und zur Modifikation bestimmter Verhaltensweisen genutzt. Je nach Erfahrungsschatz und individueller Ausprägung dieser Kontrollfunktionen können verschiedene Menschen ihr Verhalten in einer Situation, die Initiative erfordert, unterschiedlich gut steuern. Als diejenige Region des menschlichen Gehirns, die sich am Langsamsten ausbildet, ist der präfrontale Kortex in seiner Entwicklung auch in besonders hohem Maße durch das soziale Umfeld, in das ein Kind hineinwächst, beeinflussbar. Die dort angelegten neuronalen und synaptischen Verschaltungsmuster werden nicht durch genetische Programme, sondern durch eigene Erfahrungen herausgeformt.

Die Fähigkeit oder Unfähigkeit, sich erfolgreich Herausforderungen zu stellen, ist also keineswegs angeboren oder gar zufällig. Metakompetenzen werden durch Lernprozesse gewonnen, die auf Erfahrung beruhen. Wie gut ihre Ausformung gelingt, liegt somit in der Hand derer, die das Umfeld eines jungen Menschen prägen und mit inm in einer emotionalen Beziehung stehen. (HÜTHER 2009: 105)
\end{abstract}

In den nächsten Jahren kann somit vermutlich mit Spannung erwartet werden, wie die Entwicklung der Neurobiologie mit der Didaktik zusammenfließt und sich gegenseitig befruchtet.

60 An dieser Stelle lohnt sich der Hinweis das im folgenden zitierte Werk (Hermann, U. (Hrsg.), Neurodidaktik, Grundlagen und Vorschläge für gehirngerechtes Lernen, 2. Aufl., Beltz, Weinheim/Basel, 2009) näher zu betrachten, da es aktuelle neurobiologische Befunde enthält, die äußerst ertragreich in der Didaktik angewendet werden könnten. 


\section{Neurobiologie und Stress}

Da im Rahmen dieser Arbeit auch versucht wird die Idee des 'Infokoffers' in Ansätzen neurodidaktisch zu erklären, folgt nun ein Kapitel, in dem die Zusammenhänge von Neurobiologie und Stress erörtert und dabei schon erste Verbindungen zur Lernfähigkeit gezogen werden.

\subsection{Definition(en)}

Unter Stress versteht man nach JANKE et al (JANKE ET AL 1974, 1976) ein psycho-somatischehs Geschehen. Dieses weicht bezüglich der Intensität sowie Dauer vom Normalzustand ab, welche jeweils individuell ist. Umgangssprachlich wird Stress synonym mit Belastung oder einer Belastungssituation gebraucht. Im nicht formalen Sinn kann diese Abweichung vom Normalzustand neben einem psycho-somatischen Geschehen auch als Prozess der Veränderung hinsichtlich Verhalten oder dem Erleben beschrieben werden - letzteres auf primär psychischer Ebene.

Erregtheit und Spannung aber auch andere spezifische emotionalmotivationale Veränderungen stellen die psychische Komponente dieses Geschehens dar. In Zusammenhang damit stehen auch bestimmte somatische Veränderungen, genauer gesagt Aktivitätsveränderungen im zentralen und peripheren Nervensystem, in neurochemischen Systemen, sowie dem Stoffwechsel- und Immunsystem.

Diese Reaktionen auf Stress können zugleich als Indikatoren für Stressgeschehen betrachtet werden. Die Vorgänge, die mit der Stressverarbeitung oder -bewältigung einhergehen, sind verschiedene psychische und somatische Veränderungen, die dem Versuch gleich kommen, den Ausgangszustand (vor dem Eintreten von Stress) oder aber einen neuen Normalzustand (wieder) zu erreichen.

Dieses Verständnis von Stress knüpft in gewissen Teilaspekten an HANS SELYE an, der bei der Untersuchung von Stress ebenfalls sein Augenmerk auf das innerorganismische Geschehen richtete. SELYE stellte desweiteren die Annahme auf, dass nicht nur verschiedenste psychische Zustände starker Intensität, sondern auch Bestrahlungen, Verbrennungen, Infektionen oder auch 
Intoxikationen ein derartiges somatisches Geschehen nach sich ziehen. Dieses Geschehen beschreibt SELYE als eine Reihung sequentiell auftretender Reaktionen, welche über Hypothalamus, Hypophyse und Nebennierenrinde vermittelt werden.

SELYE differenziert diese Reaktion in 3 Stadien, nämlich die Alarmreaktion, das Widerstandsstadium und das Erschöpfungsstadium.

Selyes Stresskonzept betont die Bedeutung von Glucocorticoiden, Hormonen der Nebenniere, die als Reaktion auf verschiedenste Arten von Stressoren ausgeschüttet werden. Sein Konzept entwickelt damit die Theorie von WALTER CANNON weiter, welche bereits rund 30 Jahre vor SELYE die somatischen Veränderungen bei Schmerz, Hunger, Furcht und Ärger untersucht hatte und dabei die Bedeutung von Sympathikus und Nebennierenmark für alle Emotionen betonte und ähnlich wie SELYE keine spezifischen Verknüpfungen o.g. feststellt (CAnNon, 1915, rev. Auflage 1929). Selye hält weiter fest, dass die sogenannte Notfallreaktion, also das physiologische Geschehen, welches in Folge einer gestörten Homöostase zu beobachten ist, einen Vorgang darstellt, der den Energiebedarf für lebensnotwendige Handlungen reguliert.

Mit Selyes Veröffentlichungen in den 30er Jahren (Selye 1936, 1950) fand der Stressbegriff auch einen Einzug in verschiedene Humanwissenschaften, was zu einer großen Anzahl von teilweise oder gänzlich widersprüchlichen Stressdefinitionen führte.

Einige Elemente jedoch traten wiederholt in verschiedenen Stressdefinitionen auf und sollen hier kurz in Auszügen dargestellt werden:

In den Definitionen von Cannon (1935), Selye (1950) Holmes und RaHe (1967) sowie McEWEN und MENDELSON (1993) wird Stress als ein von der Homöstase verschiedener Zustand aufgefasst.

Bei Selye (1946) findet sich die oben bereits erwähnte Festlegung, dass Stress eine unspezifische Reaktion auf jede Art von Anforderung durch den Organismus bedeutet.

LAZARUS hingegen spricht (1966 und 1984 zusammen mit FOLKMAN) von einer Reaktion auf Bedrohung.

Für MCGRATH (1970) wird Stress durch Überforderung ausgelöst. 
JANKE (1974 sowie zusammen mit WoLfFGRAMM 1995) und SAPOLSKY (1992) sprechen von Stress als einem längerfristig und stark von der „Normal“Lage abweichenden Zustand.

Die beiden erstgenannten (JANKE 1974 und JANKE und WOLFFGRAMM 1995) führen weiter aus, dass Stress zu Abweichungen von der intraindividuellen 'Normal-Lage' führen kann, die dennoch recht unspezifisch ist und in Aktiviertheit und Erregtheit mündet. Außerdem führen sie an, dass es zu psychosomatischen Geschehen kommen kann, welche entweder von hoher Intensität sind oder langanhaltend.

LAZARUS und FOLKMAN (1984) definieren Stress als ein bestimmtes Verhältnis zwischen Person und Umgebung, welches das Wohlbefinden gefährdet und von der Person als mit den vorhandenen Ressourcen nicht zu bewältigen wahrgenommen wird. Stress stellt für LAZARUS und FOLKMAN (1984) einen prozesshaften Vorgang dar, welcher sich über die Zeit hinweg hinsichtlich seiner Stärke und Beschaffenheit verändert.

Auch BAUM und SINGER (1987) sprechen bei Stress von einem Prozess, wobei sich dieser dadurch charakterisieren lässt, dass bestimmte Umweltbedingungen den Organismus hinsichtlich seiner Existenz bzw. seines Wohlergehens bedrohen.

Bei BREZNITZ und GoldBeRger (1993) lässt sich ein Hinweis darauf finden, dass Stress nicht nur durch von außen auftretende Belastungen, sondern auch durch im inneren entstehende Belastungen hervorgerufen werden kann.

KASL (1996) schließlich spricht von einem die Gesundheit beeinträchtigendem Geschehen wie vom Gleichgewicht abweichende Zustände (CAnnon, 1935; Selye, 1950; Holmes und RaHe, 1967; McEWEn Und Mendelson, 1993). Nach LAZARUS (1966 und LAZARUS und FOLKMAN 1984) können durch Bedrohung ausgelöste Geschehen oder auch nach SELYE (1936) von Reaktionen des Organismus die unspezifisch ausfallen und auf jede Art von Anforderung hin auftreten.

Weitere Beschreibungen von Stresszuständen sind neben den bereits oben genannten: von der 'Normal-Lage' abweichender Zustand (JANKE 1974, JANKE und 
WOLFFGRAMM 1995, SAPOLSKY 1992), Geschehen, die nach MCGRATH (1970) das Individuum überfordern, oder auch nach BREZNITZ und GOLDBERGER (1993) Geschehnisse (endogener oder exogener Natur), die durch Überforderung und Belastung auftreten. Nach LAZARUS und FOLKMAN (1984) können Beziehungen eingegangen werden zwischen Person und Umwelt, die das Wohlbfinden des Indivduums gefährden und zudem als überfordern wahrgenommen werden.

Oben genannte Gedanken nach KASL (1996) weiterführend treten weitere gesundheitliche Beeinträchtigungen auf.

Im weiter gefassten Gesundheitsfokus können nach BAUM und SINGER (1987) auch Prozesse zu Tage treten, die den Organismus über Umweltbedingungen schädigen oder gar eine Herausforderung für Wohlergehen und Existenz des Inividuums darstellen.

Zusammenfassend lässt sich sagen, dass Stressdefinitionen, die ihren Ursprung in der psychologischen Richtung haben mit SELYES Konzeption nur wenig Übereinstimmung zeigen. So können viele Definitionen der Psychologie dadurch charakterisiert werden, dass der Bezugsrahmen ein sehr eng gefasster Phänomenbereich ist (beispielsweise Gegebenheiten, welche durch Leistungsdruck gekennzeichnet sind oder aber Situationen, die das Selbstkonzept in Frage stellen sowie auch solche, in denen das Subjekt mit Ängsten konfrontiert wird, die von außen her auftreten).

Die Betonung des Zusammenhangs von Stress und negativen Emotionen, die seit den Veröffentlichungen von LAZARUS (1966) prädominant ist, muss angezweifelt werden, da auch emotional neutrale oder positive Situationen mit Stressphänomenen in Verbindung gebracht werden können.

Als Fazit bleibt damit an dieser Stelle anzumerken, dass keine disziplinübergreifende gültige Definition von Stress existiert, was sich vor allem durch die unterschiedlichen Blickwinkel, aus denen das Phänomen betrachtet, wird begründet. Gleiches gilt auch weitestgehend intradisziplinär, womit verständlich wird, dass verschiedene Autoren nahe gelegt haben, den Begriff komplett aufzugeben.

Stress steht begrifflich in engem Zusammenhang mit den Auslösern von Stress, also Stressoren, die sich als bestimmte Umweltbedingungen und seltener auch als 
bestimmte verworrene, innere Zustände charakterisieren lassen (vgl. auch GOLDBERGER UND BREZNITZ 1993, bzW. vgl. oben).

Beim Charakterisieren von Stressoren ergibt sich jedoch ein erhebliches Problem, da kaum Stressoren existieren, die immer "Gültigkeit“ besitzen. Das heißt, es gibt viele vermeintliche Stressoren, die nicht bei allen Personen zu Stressreaktionen führen oder bei ein und der selben Person nicht Stressreaktionen auslösen (als Ausnahme können hier evtl. extreme Situationen gelten, wie etwa Misshandlung oder Lebensbedrohung).

Dieses Problem der Gültigkeit kann zwar eingegrenzt werden, wenn Stressoren in Experimenten eingesetzt werden, jedoch ist auch hier die Beschreibung und objektivierte Erfassung zumindest teilweise ein schwieriges Unterfangen.

Daher werden sowohl Selbst- wie auch Fremdberichte des öfteren eingesetzt, um Stressoren zu kennzeichnen. Erstere kommen besonders bei der Lebensereignisforschung zum Einsatz, einem Ansatz, der vor allem durch die Arbeit von HOLMES und RAHE (1967) vermehrte Aufmerksamkeit erfahren hat. Dieser Forschungsansatz ermöglicht es, die durch die Untersuchten selbst über einen längeren Zeitraum aufgezeichneten belastenden Lebensereignisse zu betrachten und damit Inventare potentieller Stressoren zu erstellen.

Letztendlich bleibt natürlich auch die Verwendung von Selbstberichten nicht unproblematisch und dabei besonders der Aspekt der Kontamination von Stressor und Stress wie er etwa auftritt, wenn subjektive Daten zur Ermittlung von Stressreaktionen verwendet werden.

Als ebenso problematisch kann der Versuch gelten Stressoren objektiv zu kennzeichnen, wenn die Grundlage dieser Kennzeichnung (zwangsläufig) subjektiv verzerrte Selbstberichte bilden, wobei angemerkt werden muss, dass subjektive Faktoren wohl miteinbezogen werden müssen, um die Auswirkungen eines Stressors vorhersagen zu können.

Ausführlicher erörtert wurden diese Probleme beispielsweise bei FRESE (1989), Frese und ZAPF (1988), GoldBerger und BREZNitZ (1982, 1993), KASL (1996) und KASL und COOPER (1987).

Wie oben bereits angedeutet, zeichnen sich Stressoren unter anderem durch ihre immense Vielfalt der zu Grunde liegenden Bedingungen und deren 
Wirksamkeit aus. Dies erschwert es natürlich auch Gemeinsamkeiten für die hervorgerufenen Reaktionen zu erörtern, das heißt eine allgemeine Veränderung zu bestimmen, die als Stress bezeichnet werden kann.

Aufgrund dessen soll an dieser Stelle auch noch darauf verwiesen werden, dass eben diese Reizbedingungen, die hier als Stressoren bezeichnet werden, in komplexen Zusammenhang stehen, da ihre Wirkung für gewöhnlich über multiple Komponenten transportiert wird, wobei diese wiederum sowohl eigenständig als auch im Verbund Wirksamkeit zeigen können.

Somit muss also auch darauf verwiesen werden, dass additive wie auch multiplikative Wirkungszusammenhänge bei zeitgleichem Auftreten mehrerer Stressoren zu beobachten sind.

Im Folgenden soll die Komplexität von Stressoren nochmals beispielhaft schematisch erläutert werden: Bestimmte kritische Lebenereignisse (HOLMES und RAHE, 1967) können als Stressperioden auftreten, die sich wiederum in mehrere Komponenten differenzieren lassen. Verschiedene Komponenten, die in zeitlicher Reihung auftreten, können sich in ihrer Wirkung verbinden und damit als umfassenderer Stressor wirken.

Aus diesem Grund, dass sich Stressoren also aus mehreren Komponenten zusammensetzen können und diese Zusammensetzung sich wiederum als über die Zeit hinweg in ihrer Qualität veränderlicher Prozess verstanden werden kann, müssen die Kombinationswirkungen meistens durch ausführliche Analysen bestimmt werden.

So werden selbst in experimentellen Untersuchungen häufig (unnötig) komplexe Stressoren verwendet, beispielsweise im Trierer Sozialen Stresstest (TSST) (vgl. Kirschbaum, PIRKe und Hellhammer 1993) in dem körperliche, mentale, emotionale Belastung und Beanspruchung hervorgerufen werden und schließlich mit einer Verletzung des Selbstkonzeptes kombiniert werden.

Hierzu muss auf ERDMANN und BAUMANN (1996) verwiesen werden, die gezeigt haben, dass allein das im TSST geforderte Halten einer öffentlichen Rede durch die darin eingebundenen Komponenten (Antizipation öffentlicher Bewertung, Vorbereitung des Themas) einzeln und in Verbindung miteinander zu unteschiedlichen Wirkungen führen.

Ein weiteres Beispiel für einen komplexen Stressor stellt der in der Biologie häufig untersuchte Rangkampf (vgl. HOLST, FUCHS und STÖHR, 1983, HENRY und 
STEPHENS 1977) dar.

\subsection{Auswirkung im Verhalten}

Stressbelastungen wirken sich individuell aus. Durch Rückgriff auf hirnbiologische Forschung kann man neuronale Prozesse seit dem Aufstreben der Hirnforschung erklären und davon Abstand nehmen das Verhalten eines Individuums als eine Art 'Black Box' zu sehen:

Because of advances in neuroscience, we are now in a better position to understand the extensive neurobiological nature of this neuronal infrastructure - even the dynamic orchestra of genetic transcriptions that help sustain mental life, with potential new concepts in genetic medicine around the corner (Hannon, 2002 nach LIOTTI und PANKSEPP 2004: 66)

LIOTTI und PANKSEPP halten fest, dass affektive Zustände nur schwer zu regulieren sind, da diese letztendlich in sehr alten Hirnregionen entstehen und eine spontane 'Handlungsintentionalität' besitzen, welche die Grundlage für höhere kognitive Fähigkeiten darstellt. Sie betonen weiter, dass die feinkörnige Forschung der Neurowissenschaften, etwa in der Untersuchung der Amygdala, nur einen kleinen Teil des Gesamtbildes abbilden, da diese Struktur, welche bestimmte äußere Ereignisse mit Angsttendenzen verbindet, nicht wesentlich für Angstgefühle ist.

Auf Grund von Fortschritten der Neurowissenschaften verbessern sich die Möglichkeiten zum Verständnis der extensiven neurobiologischen Natur der neuronalen Infrastruktur - selbst 'das dynamische Orchester' der genetischen Übertragung, die zum Erhalt des geistigen Lebens beitragen und mögliche neue Konzepte für genetische Medizin in Aussicht stellen.

HÜTHER und SACHSSE beschreiben die neuronalen Prozesse beim alläglichen Verhalten anhand von hirnbiologischen Ansätzen und zeigen damit, wie die Neurowissenschaften Verhalten erklärt:

\footnotetext{
Die Intensität der Aktivierung von Alarmreaktionen im Gehirn hangt einerseits vom Ausmaß der wahrgenommenen und zum Gehirn weitergeleiteten Störung ab, andererseits von der aufgrund von Vorerfahrungen im Gehirn zur Bewältigung dieser Störung verfügbaren Reaktionen und Antworten. Letztere bestimmen darüber, wie die eingetretene Störung, nämlich das im Gehirn entstandene Alarmsignalmuster bewertet wird. Im Gegensatz zu den Tieren spielt diese Bewertung beim Menschen eine ausschlaggebende Rolle für das Ausmaß der subjektiv empfundenen Bedrohung. Damit haben beim Menschen nicht nur die objektive Qualität und Quantität der Gefahr, sondern auch die subjektive Bewertung derselben einen erheblichen Einfluss auf das Ausmaß und die Intensität der durch die weitergeleitete Störung tatsachlich im Gehirn ausgelosten Alarmreaktion. (HÜTHER und SACHSSE 2007: 167f)
} 
Weiterhin erläutern HüTHER und SACHSSE den hirneigenen Schutzmechanismus in Gefahrensituationen, der zudem auch die subjektive Bewertung und die Intensität der Reaktion steuert. Dafür sind im Gehirn zwei Systeme vorhanden: das Panik- und das Furchsystem, welche insbesondere in Stresssituationen greifen:

Das Paniksystem reagiert mit Disstressempfindungen, wenn es uns nicht gut geht und wir uns hilflos-ohnmächtig wie Säuglinge oder Kleinkinder mutterseelenallein in der Fremde fühlen. [...]

Dies sind andere Zentren als die, die bei der Furchtreaktion aktiviert werden. Ab einem bestimmten Alter wird es freilich immer sinnloser, nach Mama zu rufen, weil Mama nicht mehr käme. Das Paniksystem wird so zunehmend durch neue Vernetzungen überlagert, weil es nicht mehr gebraucht wird, weil seine Aktivierung ineffektiv wird. Aber auch schon für ganz junge Tiere kann es problematisch sein, nach Mama zu rufen. Dies gilt für jene Situationen, in denen ein Raubtier in der Nahe ist, Mama zu weit weg oder Mama zu schwach ist. Dann ist es funktionaler, möglichst still zu sein, sich unbeweglich zu machen, sich ganz eng an die Erde zu pressen und keinen Mucks von sich zu geben.

(HÜTHER und SACHSSE 2007: 168)

Das Phänomen des 'möglichst still sein' (das sogenannte 'Freeze' Phänomen aus der Trias: Freeze, Fight und Flight $\rightarrow$ Still halten/sich tot stellen, kämpfen oder flüchten) ist auch auf den generellen Lebensalltag der Migranten übertragbar. Bei gleichzeitiger innerer 'hyperarousal', also Übererregung, weil die Migranten 'fern der Heimat' leben und damit große Anforderungen an sie gestellt werden im Zielland, versuchen sie sich gleichermaßen anzupassen und nicht negativ aufzufallen. Dieses Wechselspiel führt wie bei den oben zitierten Raubtieren in manchen Fällen dann zu einem Erstarren auf verschiedenen Ebenen. Im Gegensatz zum Paniksystem ist das Furchtsystem zudem gegenwärtig weit besser untersucht:

Das Furchtsystem und seine Störungen (Yehuda 2000; Sachsse et al. 2002) sind viel besser erforscht als das Paniksystem, das erst in letzter Zeit in den tierexperimentellen Blickpunkt gerat und ja auch für die Bindungsforschung beim Menschen erhebliche Bedeutung hat. Das Furchtsystem ist mit Kampf und Flucht, mit "fight and flight" verbunden, seine Physiologie ist bekannt, und es ist die Basis für unsere kognitiven Lernschritte (Hüther 1996; Hüther 1997 nach HÜTHER und SACHSSE 2007: 168)

Obwohl das Furchtsystem bereits durch die 'fight and flight' Verbindung stärker untersucht ist, sind Panik und Furcht eng miteinander verbunden. Als Folge von Furcht kann Panik entstehen. Dies und viele weitere Aspekte, die mit diesem System zusammenhängen, erläutern HÜTHER und SACHSSE ausführlich (und lesenswert) auf hirnbiologischer Ebene: 
- sehr eng miteinander verbunden und interagieren intensiv. So ist das Furchtsystem ein wichtiges Beruhigungssystem für das Paniksystem. Durch Einsicht, Kognition und Lernen können wir uns aus der Situation der diffusen Panik herausbegeben. Das versuchen wir auch, und dies versuchen auch die Tiere.

Wir machen uns Furcht, wenn wir eigentlich Panik haben, weil wir mit Furcht viel besser umgehen können, weil wir die Motorik einsetzen können, kämpfen oder fluchten können, und weil wir dann unsere Kognitionen nutzen können.

Der Mensch ist ein ganz besonderes Tier - vor allem, was seine Fähigkeit zur Stressbewältigung betrifft. Mit seiner enormen Ausdehnung des Kortex geht die Fähigkeit zur Verknüpfung verschiedener Wahrnehmungen, zur Verankerung von Erfahrungen, zur Ausbildung eines enormen autobiographischen Gedächtnisses, zum Erlernen und Abspeichern von Sachwissen im expliziten Gedächtnis und nicht zuletzt zur Herausbildung so genannter Metakompetenzen, wie Selbstwirksamkeitskonzept, Handlungsplanung, Impulskontrolle, Folgenabschatzung, Frustrationstoleranz, und Metakognitionen, wie Vorstellungen, Ideen, Überzeugungen, einher (Hüther 2004). Vieles, was Tiere sofort in Furcht und Panik versetzt, lasst die meisten Menschen kalt, weil sie das Geschehen, besser die im Hirn eintreffende Wahrnehmung, die normalerweise eine Alarmreaktion in Gang setzen wurde, zunächst einmal als gefahrlos bewerten. Natürlich ist das bisweilen eben auch falsch. Was dann von Panik und Furcht übrig bleibt, ist im einen Fall Beunruhigung, im anderen Irritation.

$[\ldots]$

Erst wenn all das, was von dort aus an Erfahrungen, Erinnerungen, Vorstellungen, auch an Hoffnung und Zuversicht, durch die Aktivierung entsprechender neuronaler Netzwerke und komplexer Verschaltungsmuster wachgerufen werden kann, nicht ausreicht, um die sich ausbreitende Unruhe im Zaum zu halten, also die unspezifische Erregung zu unterdrucken, (Merke: die wichtigste Funktion des Kortex ist Hemmung!), kommt es zur Aktivierung der tiefer liegenden, subkortikalen Bereiche.

Das Alarmsystem wird also bei uns erst eingeschaltet, wenn wir am Ende unserer Kunst angekommen sind. Das dauert normalerweise nicht nur deutlich länger als bei anderen lernfähigen Tieren.

Es ist auch von Mensch zu Mensch sehr unterschiedlich und hangt davon ab, wie viel Kunstfertigkeit jemand im Umgang mit schwierigen Situationen bereits besitzt oder sich aneignen konnte.

$[\ldots]$

Wie aus der Darstellung der Alarmreaktionen bereits deutlich geworden ist, sind die durch Bedrohung der inneren Ordnung ausgelosten Reaktionen nicht von jenen zu trennen, die zur Wiederherstellung des gestörten Gleichgewichts eines Organismus im Körper und im Gehirn aktiviert werden. Alle Stresssysteme sind selbstverständlich immer StressBewältigungs-Systeme.

(HÜTHER und SACHSSE 2007: 170f.)

In diesem Kontext bietet es sich an, nochmal den Rückbezug zum Thema Identität herzustellen. Durch den Wegfall der Heimat für die Migranten als deren Sicherheit in Kombination mit dem Bedürfnis des 'Nicht auffallen wollens' im Zielland und weiterhin der Suche nach Identifikationsangeboten könnte man somit von der Identitätssuche in der 'Fremde' als von einem 'Stress-BewältigungsSystem' sprechen, welches zum Beispiel die Herde (Familie) auslösen kann. Somit kann Identität als Sicherheit (Ich-Stabilität) verstanden werden. Der Schluss liegt nahe, dass, umso fremder sich ein Individuum im Zielland fühlt, desto mehr Nähe und Sicherheit nötig sind und gesucht werden. Im Falle des Nichtvorhanden-seins einer 'Herde' muss das Individuum seine Selbst-Stärke ausbauen

(vgl. Stichwort: Selbstwirksamkeitskonzept). Durch die Identitätssuche zwischen 
Erwachsenen-Ich-in-Deutschland, das mit 'der Realität' konfrontiert ist, und dem intrapsychisch 'regredierten Ich' (das die Heimat idealisiert) entsteht eine Ambivalenz:

Gerade dieser Situation der gleichzeitigen Aktivierung der bewussten Erwachsenenebene und der regressiv-erlebenden kindlich-pathologischen Ebene versuchen die Patienten auszuweichen oder sich zu entziehen. Sehr viele sind entweder erwachsen oder regressiv, entweder arbeitsfähig oder in einem pathologischen Zustand, denn die gleichzeitige Aktivierung und Wahrnehmung dieser beiden Zustande führt zu Schmerzen, Leid, Ambivalenz, Zwiespalt und anderen aversiven Empfindungen.

(HÜTHER und SACHSSE 2007: 170)

Eine Ambivalenz erleben auch Flüchtlinge und Vertriebene. BEUTEL et al. verweisen in diesem Kontext auf Befunde, die dafür sprechen, dass Flucht- und Vertreibungserfahrungen erhebliche psychosomatische Langzeitwirkungen zur Folge haben, welche unter anderem in Form verdrängter Erfahrungen, die u.U. auch durch erhöhte Medienpräsenz aktueller Flüchtlingskatastrophen reaktiviert, wirksam sein können (BEUTEL et al. 2007: 206). Die Autoren konnten in ihrer Untersuchung signifikante Unterschiede in der Lebensqualität zwischen Vertriebenen und Nichtvertriebenen feststellen (unter anderem für „Körperliche Funktionsfähigkeit“ und „Vitalität“ sowie bei „körperlichen Rollenfunktionen“ und „Sozialer Funktionsfähigkeit“, tendenziell auch für „Allgemeine Gesundheitswahrnehmung.") (BEUTEL et al 2007: 209).

Einen weiteren interessanten Aspekt untersuchte SAPOLSKY. Er beantwortet die Frage, wie gealterte Organismen mit Stress zurecht kommen, folgendermaßen:

Nicht sehr gut, wie sich herausstellt. In vieler Hinsicht kann Altern als fortschreitender Verlust der Fähigkeit definiert werden, Streß zu bewältigen, und das entspricht sicherlich unserem Bild von älteren Menschen als gebrechlich und anfällig. Genauer ausgedrückt könnte man sagen, daß alte Organismen körperlich und geistig/seelisch genauso gut wie junge funktionieren - solange sie nicht besonderen Belastungen ausgesetzt sind. Kommen jedoch sportliche Herausforderungen hinzu, eine Verletzung oder Krankheit, Zeitdruck oder neue Umstände - irgendeiner der verschiedensten körperlichen, kognitiven oder psychologischen Stressoren -, dann versagen gealterte Organismen schneller.

Die Abbildung auf Seite 290 zeigt dies in schematischer Weise. In Abwesenheit von Streß (welcher Art auch immer) sind junge und alte Personen etwa gleich leistungsfähig. Unter Streß nimmt die Leistung in beiden Altersgruppen ab - bei alten Menschen jedoch schneller. (SAPOLSKY 1994: 289)

Stressreaktionen können einer der Gründe dafür sein, dass ältere Menschen mit somato-psychischen Reaktionen beschäftigt sind:

Ältere Menschen sind also in vieler Hinsicht genauso leistungsfähig wie jüngere, solange sie nicht gestreßt sind. Sobald jedoch ein Stressfaktor auftritt, zeigt sich eine gravierende Störanfälligkeit. Viele dieser relativen Funktionsausfälle sind durch die verschiedensten Probleme mit den individuellen, an der Streßantwort beteiligten Hormonsystemen zu 
erklären. In gewisser Hinsicht ist die Stressantwort nicht ausreichend. So scheiden alte Menschen bei körperlicher Anstrengung zwar sehr viel Adrenalin und Noradrenalin aus (de facto weitaus mehr als junge Menschen). (SAPOLSKY 1994: 294)

Fortwährende Stressreaktionen können auch in 'Nicht-akuten-Stressphasen' bei alten Migranten zu Krankheiten führen und daher Phänomene, wie dauerhaft hoher Blutdruck trotz Ruhephasen, durch vormals langanhaltende Stressreaktionen (Akkulturationsstress) neurobiologisch erklärt werden:

\begin{abstract}
Alte Individuen aller Spezies neigen dazu, ihre Streßreaktion einzuschalten, selbst wenn gar nichts Stressiges passiert. Bezahlen alte Organismen einen Preis für diese „normale“ hormonelle Überaktivität? Es scheint so. Zum Beispiel haben die erhöhten Ruhekonzentrationen von Adrenalin und Noradrenalin wahrscheinlich viel mit dem erhöhten Blutdruck zu tun, den man bei älteren Individuen oft sieht. (SAPOLSKY 1994: 325)
\end{abstract}

Die bisherigen Ausführungen waren jedoch bisher nur die Auswirkungen des Stresses auf der individuellen Ebene, im nächsten Kapitel erfolgt ein Streiflicht über die Auswirkungen auf das soziale Gefüge und auf soziale Gruppen.

\title{
4.3 Soziale Auswirkungen von Stress (Gruppen- und „Herden“-Verhalten)
}

Was auf der individuellen Einzelebene greift, kommt auch auf der sozialen Ebene zum Tragen. Im Folgenden soll neurobiologisch dargelegt werden, wie Stress das Verhalten mehrerer Individuen beenflusst (Herdenverhalten und Gruppendynamik). SAPOLSKY beschreibt das Verhalten von Gruppen, um die Wirkung eines Stressors so gering wie möglich zu halten. Dabei geht es darum, dass viele Individuen sich besser organisieren können und zufriedener sind, wenn sie im Sinne einer Maximaldefinition der Ähnlichkeiten leben. Das bedeutet, dass das Kollektiv so viele Ähnlichkeiten wie möglich hat und Schutz bietet, wie eine Herde. Dies ist eine Strategie zur Stresskompensation.

Problematisch ist dabei bei Migranten jedoch, dass die dominante kulturelle Gesellschaft Gefahr läuft, die Migranten als unnahbare Einheit wahrzunehmen. Diese Einheit wird von den Migranten zur Stressreduktion gesucht, die einhergehende Stereotype sind jedoch unerwünscht. So entsteht eine Art Dilemma-Zirkel:

Es gibt noch eine Möglichkeit, wie wir mit anderen Organismen interagieren können, um die Wirkung eines Stressors auf uns gering zu halten, eine Möglichkeit, die für die Zukunft 
unseres Planeten weitaus ermutigender ist als die Aggressionsverschiebung. Ratten machen selten Gebrauch davon, aber Primaten können das wunderbar. Man füge einem Affenjungen irgendetwas Unangenehmes zu: es bekommt eine Streßreaktion. Man unterwerfe es dem gleichen Stressor, während es in einem Raum voll anderer Primaten ist - jetzt kommt es darauf an. Sind diese Primaten Fremde, dann verstärkt sich die Streßantwort. Aber falls es sich um Freunde handelt, läßt die Stressantwort rasch nach. Unterstützende soziale Netzwerke - es hilft einem, eine Schulter zu haben, an der man sich ausweinen, eine Hand, die man halten kann, ein Ohr, das einem zuhört, jemanden, der uns in die Arme nimmt und uns sagt, daß alles wieder gut werden wird.

Manchmal reicht dazu schon ein erstaunlich geringer sozialer Kontakt aus. Bei einer wohldurchdachten Demonstration wurde die hormonelle Streßreaktion von Menschen studiert, die sich einer schmerzhaften und angstbesetzten Herzkatheterisierung unterziehen mußten.

Bei denjenigen, die während der Prozedur mit ihren Ärzten über ihre Furcht sprachen, traten geringere Glukokortikoid- Streßantworten auf als bei den Stoikern. (SAPOLSKY 1994: $231 \mathrm{ff})$.

Weitere Möglichkeiten, Stress in der Gruppe zu reduzieren, sind die antizipierte Nähe und die damit verbundene Kontrolle:

Die Variable "Kontrolle" ist äußerst wichtig; die Kontrolle über die Belohnungen zu haben, die man bekommt, kann erwünschter sein, als die Belohnung einfach so zu kriegen.

[...]

Fassen wir zusammen: Streßantworten können von psychologischen Faktoren einschließlich dem Verlust von Frustrationsventilen und von sozialer Unterstützung, dem Eindruck, daß sich die Situation verschlechtert, und, unter Umständen., dem Verlust von Kontrolle und Vorhersagbarkeit modifiziert oder sogar verursacht werden. (SAPOLSKY 1994: 237).

Allerdings ist nicht jede soziale Bindung hilfreich, um Stress zu reduzieren. Die eingangs erwähnte Ähnlichkeit ist elementar, genauso wie - in einer Hierarchie - das Zusammensein mit Menschen auf der gleichen oder nächstähnlichen Stufe ist hilfreich, um Stress zu reduzieren. Für Migranten bedeutet dies, das ungünstige Alleingelassen werden oder Alleinsein in einer fremden Umgebung:

Soziale Bindung ist nicht immer die Lösung bei seelischen Erschütterungen.

Wir alle kennen genügend Menschen, auf die wir in Zeiten seelischer Not um keinen Preis angewiesen sein möchten. Wir können uns seelische Belastungszustände vorstellen in denen das Zusammensein mit jedem Menschen unser Befinden verschlechtern würde. Auch physiologische Untersuchungen haben dies bestätigt. Wenn man ein Nagetier oder einen Primaten nimmt, die allein untergebracht waren, und sie einer sozialen Gruppe hinzugesellt, ist das typische Ergebnis eine massive Streßreaktion. Im Fall von Affen kann diese wochen oder monatelang andauern, während die Tiere angespannt herauszufinden versuchen, wer wen in der sozialen Hierarchie der Gruppe dominiert*. Bei einer anderen Demonstration dieses Prinzips wurden Affenjunge von ihren Müttern getrennt. Wie vorauszusehen, hatten sie beträchtliche Streßreaktionen mit erhöhtem Glukokortikoidspiegel. Dieser Anstieg konnte verhindert werden, wenn das Junge einer sozialen Gruppe von Affen hinzugesellt wurde - aber nur wenn das Junge diese Tiere bereits kannte. Von Fremden hat man wenig Tröstung zu erwarten.

Selbst nachdem die Tiere einander nicht mehr fremd sind, ist im Schnitt die Hälfte der Mitglieder jeder sozialen Gruppe sozial dominant gegenüber einem bestimmten Individuum, und viele dominante Tiere um sich zu haben ist in schwierigen Zeiten nicht unbedingt ein Trost. Selbst intime soziale Bindung ist nicht immer hilfreich. (ebd. ff) 
Zu einer ähnlichen Thematik erläutert FAUSER, dass MAURICE HALBWACHS in seinem Hauptwerk der Frage nachgeht, was eine soziale Gruppe zusammenhält, nämlich u.a. auch die Gleichheit in der Erinnerung (Gruppengedächtnis an kulturelle Narrative) können hilfreich sein, um sich unter seines Gleichen zu fühlen:

Dabei stößt Halbwachs auf die konstitutive Rolle der gemeinsamen Erinnerungen, ohne die
eine Gruppe kaum existieren könte. Aber das Gruppengedächtnis ist nicht nur ein Teil
unserer Existenz, sondern das Fundament, auf dem jedes Individuum seine innere
Erinnerung baut. Das erfahren wir selber, wenn wir feststellen, dass die meisten unserer
Erinnerungen dann kommen, wenn andere sie uns ins Gedächtnis rufen (Halbwachs 1966
S.20). Andererseits sind unsere angeblichen Erinnerungen sehr oft Erzählungen entlehnt,
die wir von anderen gehört haben. Und unsere Erinnerungen fügen sich auch in kollektive
Erzählungen ein, den kulturellen Narrativen auf allen Ebenen von Alltag bis zu den
offentlichen Handlungen. Es gibt also einen großen Bereich von gemeinsam geteiltem
Bewusstsein und zwar sowohl was die Inhalte, als auch die Strukturen betrifft, in denen sie
sich mitteilen. Halbwachs interessiert die Beschreibung dieser "cadres sociaux“, also der
"sozialen Rahmen“, wie man übersetzen könnte, in denen sich das Gedächtnis bewegt:
Die Rahmen, von denen wir sprechen und die uns die Rekonstruktion unserer
Erinnerungen nach ihrem Verschwinden erlauben, sind nicht rein individuell; sie sind, wie
wir sagten, den Menschen der gleichen Gruppe gemeinsam. (Halbwachs 182f.
nachFAUSER 2008: 117f)

Gesamtgesellschaftlich ist also eine Anti-Panik-Haltung gegenüber 'Fremden' nötig, um für die Migranten eine 'Sicherheitsvermittlung' zu schaffen, damit sie sich auch aus der Herde der anderen Migranten herauswagen und Ähnlichkeiten mit den 'Fremden' der dominanten Kultur entdecken können. Auf der anderen Seite fördert eine Anti-Panik-Haltung auch den stressfreien Rückzug in die Herde und vermittelt ein Sicherheitsgefühl, welches sich zu einem Anti-FurchtSystem entwickelt und damit Lernen möglich macht.

Es ist wichtig, Quellen sozialer Bindung und Unterstützung zu finden. Selbst in dieser extrem individualistischen Gesellschaft sehnen sich die meisten von uns danach, sich als Teil von etwas zu fühlen, das größer ist als wir selbst. Aber man sollte echte Bindung und Unterstützung nicht mit bloßer Geselligkeit verwechseln. Man kann sich äußerst einsam in einer Menschenmenge oder im Umgang mit einem vermeintlich intimen Freund oder einer Vertrauensperson fühlen, die sich als Fremde erwiesen. (SAPOLSKY 1994: 349).

Vertrauenspersonen sind in der Fremde essentiell. Somit muss eine der Forderungen sein, um älteren Migranten Bildung zu emöglichen, ein Bildungssystem zu schaffen (Stichwort salad bowl), welches ihr Herdenverhalten vorurteilslos zulässt und ihnen ermöglicht, es zu praktizieren, um ihnen damit die nötige Sicherheit und Identität zu verschaffen. Damit würde die Identität aufrecht erhalten werden können und somit im letzten Schritt durch die Bindung zur eigenen 'Herde' die Lernfähigkeit erhöht oder überhaupt die Möglichkeit zu Lernen 
erst geschaffen werden, da der blockierende Stress reduziert würde indem diese positive Erfahrung (Sicherheit) im Gedächtnis gespeichert würde und somit auch zur Stressreduktion beitrüge:

Es lässt sich am Zutreffendsten mit dem Begriff „Erfahrung“ umschreiben. Gemeint ist damit das im Gedächtnis eines Individuums verankerte Wissen über die in seinem bisherigen Leben entweder besonders erfolgreich oder besonders erfolglos eingesetzten, in dieser Weise immer wieder bestätigt gefundenen und deshalb auch für die Lösung zukünftiger Probleme als entweder besonders geeignet bzw. ungeeignet überbewerteter Strategien des Denkens und Handelns. Solche Erfahrungen sind immer das Resultat der subjektiven Bewertung der eigenen Reaktionen auf eine wahrgenommene und als bedeutend eingeschätzte Veränderungen der Außenwelt. Sie unterscheiden sich darin von allen (passiven) Erlebnissen und (passiv) übernommenen Kenntnissen und Fertigkeiten, denen kein oder noch kein Bedeutungsgehalt für die eigene Lebensbewältigung beigemessen wird. (HÜTHER 2005: 537-538)

Von Überlegungen zur Biologie des Einzelnengelangt HÜTHER weiter zu einer systemtheoretischen Überlegung: „Die Offenheit aller lebenden Systeme macht ihre innere Ordnung störanfällig für Änderungen ihrer Außenwelt." (HüTHER 2005: 538) Somit besteht bei äußeren Veränderungen intrapersonell die Gefahr einer Störanfälligkeit. Auf diese Weise führen wiederholte, kontrollierbare Belastungen als Konsenz zur Bahnung und zum Ausbau, also schrittweise zur Verbesserung der Effizienz der Störungen und Stressoren. Diese haben gewissermaßen bestimme Spezialisierungen, im Sinne von 'zur Routine gewordenen Reaktionen' als Folge (vgl. HüTHER 2005: 538). An dieser Stelle lässt sich SAPOLSKY (1994: 234) bestätigend zitieren:

Ein weiterer Vorzug von Vorhersagbarkeit ist, daß sich Organismen im Lauf der Zeit an einen Streßfaktor gewöhnen, falls dieser immer wieder eintritt; zwar kann die physiologische Homöostase dadurch auch beim x-ten Mal aus dem Gleichgewicht geraten, aber es handelt sich inzwischen um einen vertrauten, voraussagbaren Stressor, der nur noch eine verringerte Streßantwort auslöst.

Mit HURLEMANN lassen sich diese Erkenntnisse weiterführend direkt auf Familien-Netzwerke bei Migranten transferieren:

Diese Familien sind durch weit verzweigte familiale und verwandtschaftliche Netzwerke gekennzeichnet.

[...] wirtschaftliche Krisensituationen und Notlagen können das Familienklima und die ElternKind-Beziehungen in Migrantenfamilien strapazieren und sie zu "Risikofamilien "machen, weil meist psychische und soziale Krisen nachfolgen, die sich in lang anhaltenden Spannungen und Konflikten zwischen den Eltern niederschlagen (Schnabel 2001, S. 81). Ausgangspunkte der sozialen und psychischen Krisen können ungünstige und beengte Wohnbedingungen sowie die soziale und kulturelle Isolation der Familie und ihre Stigmatsierung sein. In den Migrantenfamilien können solche Belastungen allerdings auch oft durch ein enges Zusammenhalten und eine intensive gegenseitige Unterstützung in den gefächerten sozialen Netzwerken abgefangen werden (Beck-Gernsheim 1998). (HURLEMANN 2002: 152-153). 
Ein weiterer potentieller Sicherheitsfaktor in Bezug auf Stressvermeidung/verhinderung kann die bereits zuvor angeführte Kontrolle sein. Die Kontrollierbarkeit von Situationen in der Fremde (das Bekannte) kann auch zu mehr Sicherheit führen, während unkontrollierbare Situationen (das bedrohlich erlebte Fremde) zu Destabilisierung führen können:

\begin{abstract}
Es ist in diesem Zusammenhang bezeichnend, dass vor allem Umbruchphasen wie die Pubertät, die zu psychosozialen Neuorientierungen zwingen, besonders häufig mit lang anhaltenden, unkontrollierbaren psychischen Belastungen einhergehen. Damit tragen beide Arten von Stressreaktionen, also die kontrollierbaren Herausforderungen wie auch die unkontrollierbaren Belastungen, in jeweils spezifischer Art und Weise, zur Strukturierung des Gehirns, d. h. zur Selbstorganisation neuronaler Verschaltungsmuster im Rahmen der jeweils vorgefundenen äußeren, psychosozialen Bedingungen bei: Herausforderungen stimulieren die Spezialisierung und verbessern die Effizienz bereits bestehender Verschaltungen. Sie sind damit wesentlich an der Weiterentwicklung und Ausprägung bestimmter Persönlichkeitsmerkmale beteiligt. Schwere, unkontrollierbare Belastungen ermöglichen durch die Destabiliserung einmal entwickelter, aber unbrauchbar gewordener Verschaltungen die Neuorientierung und Reorganisation von bisherigen Verhaltensmustern. Die von unkontrollierbaren Belastungen getriggerten lang anhaltenden neuroendokrinen Reaktionen können offenbar über die von ihnen ausgelöste Destabilisierung neuronaler Verschaltungsmustern in limbischen und kortikalen Hirnregionen zu u. U. sehr grundsätzlichen Veränderungen des Denkens, Fühlens und Handelns einer Person führen. Das Ersetzen eines alten, unter dem Einfluss bisheriger Anforderungen stabilisierten assoziativen Verschaltungsmusters durch ein neues kann dazu führen, dass bisher unkontrollierbare psychosoziale Konflikte kontrollierbar werden. Ein derartiger Reorganisationsprozess ist jedoch immer mit dem Risiko der Entgleisung und des unkompensierbaren Verlustes bestimmter Fähigkeiten im Bereich des Denkens, Fühlens oder Handelns behaftet (HÜTHER 2005: 542)
\end{abstract}

Mit diesen Überlegungen lässt sich das Selbstempfinden vieler analphabetischer Migranten erklären, nach dem Integrationskurs schwerer und (vermeintlich) schlechter deutsch zu sprechen als zuvor. Dem steht beispielsweise ein Fall gegenüber, welcher unter III.2.2. noch erläutert wird: In diesem Fall lernte eine alte Migrantin intrinsisch motiviert Altarabisch, um damit den Koran lesen zu können, hatte in den Integrationskursen aber mit Schwierigkeiten des Lernens zu kämpfen, da sie, um eingebürgert zu werden, den Integrationskurs bestehen musste. Die Motivation, den Koran zu lesen, war intrinsischer Natur und fiel daher in ihrer Herde (der religiösen Gemeinschaft und Familie) deutlich leichter.

Nach HÜTHER bietet jede Krise allerdings auch eine Chance und diese Tatsache muss in beide Richtungen, als Chance und als Problem, in jedwedem didaktischen Konzept berücksichtig werden:

Schwere seelische Krisen, Verzweiflung, Ohnmacht und Hilflosigkeit bieten so eine Chance zu Reorganisation und zu einem Neuanfang. Misslingt er oder kann er nicht rechtzeitig genug eingeleitet werden, so wächst die Gefahr der Manifestation von stressbedingten psychischen oder somatischen Erkrankungen. (HÜTHER 2005: 542) 


\subsection{Akkulturationsstress}

Den Prozess der Akkulturation kann man auch als eine zweite Sozialisation, nach GEULEN (1989, 64) verstanden als „Entstehung und Entwicklung der Persönlichkeit in wechselseitiger Abhängigkeit von der gesellschaftlich vermittelten sozialen und materiellen Umwelt", auffassen.

Der wesentliche Unterschied zur ersten Sozialisation besteht freilich darin, dass die Migranten bereits „mit einer weitgehend ausgebildeten Persönlichkeit und mit umfangreichen Lebenserfahrungen aus einem anderen Lebenskontext" (RUDDAT, 1994) beschrieben sind und die Gesellschaft in die sie sich einfinden, eine andere ist, als jene, in der die ersteSozialisation stattfand.

Um die Akkulturation und den mit ihr verbundenen Stress fassen zu können ist es also nötig, die bestimmenden Element der Sozialisation, die Thomas (1993b) als komplexes Einflussgefüge aus gegenständlicher, sozialer und personaler Umwelt beschreibt, in die Betrachtung miteinzubeziehen. Um das Dasein als Migrant möglichst umfassend $\mathrm{zu}$ begreifen sollten also das innerpsychische Erleben der Migranten als Individuen aber auch der Zusammenhang zwischen den begleitenden Umständen der Migration und das aktive Formen der Existenz der Migranten durch diese selbst einbezogen werden.

In den meisten Akkulturationskonzepten wird der eben dargestellten Überlegung nicht genug Bedeutung eingeräumt.

In der stresspsychologischen Migrationsforschung, wie sie z.B. von BERRY (1989) vertreten wird, ist die Aufgabe der Migranten vor allem die Anpassung an eine neue Umwelt und somit im Wesentlichen über sozialkognitive Lernprozesse zu bewältigen.

Bestimmt werden diese Situationen also vor allem durch Wahrnehmungs-, Informationsverarbeitungs-, Bewertungs- und Urteilsprozesse, sowie durch Attributionen und Emotionen der Beteiligten.

Einen interessanten Ansatz bietet die Arbeit von TsIAKALOS (1983), der beschreibt in welcher Weise sich das Selbstkonzept der Migranten verändert, wenn diese sich als (vermeintlich) unbeliebt und ungewollt wahrnehmen. Die Folgen für das Selbstkonzept sind ein ständiges Hinterfragen, d.h. eine ständige Selbstbewertung. 
Es lässt sich also formulieren, dass das Gefühl der Unbeliebtheit zu einer stetigen Verunsicherung der Migranten führt, die, so ist zu vermuten, noch steigt, wenn in Begegnungen mit der Aufnahmegesellschaft die Möglichkeiten zur Interaktion nur eingeschränkt gegeben sind, etwa weil Sprachkenntnisse nur mangelhaft vorhanden sind, oder die Interaktion durch die Angst vor Kontrollverlust oder Diskriminierung gestört wird. Diese Steigerung der Unsicherheit wiederum kann zu emotionaler Unzufriedenheit führen und schließlich wird die Selbstwahrnehmung zusehends schlechter ausfallen.

Den aus der Akkulturation resultierenden Stress müssen die einzelnen Migranten in irgendeiner Weise verarbeiten bzw. bewältigen.

Im Folgenden werden drei verschiedene Theorien vorgestellt, in denen der Versuch unternommen wird diese Coping-Strategien zu konzeptionieren.

Der Kern der Bewältigung sind bei LAZARUS und LAUNIER (1981) verhaltensorientierte und intrapsychische Verhaltensweisen, die dazu dienen, umweltbedingte und interne Anforderungen „zu meistern, zu tolerieren, zu reduzieren oder zu minimieren“. Der Rahmen dieser Bewältigungsstrategie stellt die transaktionäre Stresstheorie nach LAZARUS und FOLKMAN (1987) dar, welche das Entstehen von Stress als Folge von externen oder internen Anforderungen, die personelle Ressourcen stark be- oder überbeanspruchen, versteht.

Wie oben angedeutet kann Bewältigung bei Lazarus also über aktives Handeln („meistern“) oder über passives Verhalten (tolerieren, vermeiden etc.) erfolgen.

LAZARUS und FOLKAN reden entsprechend von der Idee, dass es nur um das Bemühen der Individuen, mit den an sie gestellten Anforderungen fertig zu werden geht, weniger von verschiedenen Bewältigungsstrategien, als viel mehr von verschiedenen Funktionen der Bewältigung.

Die Grundannahme bei LAZARUs und FOLKMAN (1984) ist es, dass die Lebensumwelt der Menschen einem stetigen Wandel unterzogen ist. Dementsprechend stellt sich die Anforderung, die persönlichen kognitiven und verhaltensbezogenen Fähigkeiten als Erwiderung an diese sich verändernden Umstände zu entwickeln.

Die Individuen bewerten diese Anforderungen, die intern und extern an sie gestellt werden, in einem zwei Stufen umfassenden Prozess:

In der primären Bewertung legen die Individuen für sich fest, ob die 
Anforderungen möglicherweise schädlich für sie sind, in der sekundären Bewertung wird erkundet, ob die vorhanden Ressourcen, die zur Verfügung stehen, um mit dieser Situation fertig zu werden ausreichen, oder eben nicht.

Mit vorhandenen Ressourcen sind hier die individuellen Bewältigungsfähigkeiten und -möglichkeiten gemeint.

LAZARUS und FOLKMAN unterscheiden die Versuche der Bewältigung hinsichtlich des Ansatzpunktes über den die Bewältigung stattfindet.

$\mathrm{Zu}$ nennen sind dabei einerseits Bewältigungsversuche, die problemzentriert sind, also solche, die vereinfacht ausgedrückt, etwas am Problem ändern (sollen) andererseits Bewältigungsversuche, die emotionszentriert sind und dementsprechend darum bemüht sind, an der Reaktion auf die Anforderung etwas zu verändern.

Problemzentrierte Bewältigungsversuche sind also Strategien, welche das Ziel haben, die Anforderungssituation zu verändern, emotionszentrierte Bewältigungsversuche hingegen stellen das Unterfangen dar, den Emotionsgehalt einer Anforderung durch eine Veränderung in der Bewertung zu beeinflussen.

$\mathrm{Ob}$ und in wie weit diese Strategien zu einer erfolgreichen Bewältigung führen, hängt von individuellen Fähigkeiten und Möglichkeiten der Person ab.

Die Bewältigungsfähigkeiten umfassen nach HAMMERER und MARTING (1988) persönliche Ressourcen in Form eines gewissen Erfahrungsschatzes im Umgang mit vergleichbaren Anforderungssituationen, die letztlich darüber entscheiden, ob sich eine Person mit einer Situation überfordert fühlt und somit Stress erlebt und entsprechende Symptome entwickelt, oder eben nicht.

Bewältigungsmöglichkeiten hingegen beschreiben interne und externe Reaktionen einer Person in einem gegebenen Kontext auf einen bestimmten Stressor (PEARLIN und SCHOOLER 1978).

Somit ergibt sich für die transaktionale Stresstheorie von LAZARUS, dass eine Person eine bestimmte Anforderung die an sie gestellt wird in Abhängigkeit zu den wahrgenommenen Ressourcen, die ihr zur Verfügung stehen bewertet. Diese Bewertung der Situation, beispielsweise als Verlust, Bedrohung, Schaden oder Herausforderung, wirkt sich direkt darauf aus, ob durch die Anforderung für die Person Stress entsteht und wie intensiv dieser Stress ist.

In Seligmanns Theorie der erlernten Hilflosigkeit wird das Phänomen beschrieben, dass Menschen durch das wiederholte Erleben von 
unkontrollierbaren Situationen ein Gefühl der Hilflosigkeit „erlernen“, dass sich auch auf die Bewertung anderer Situationen auswirkt. Dadurch wird das Gefühl der Hilflosigkeit verallgemeinert.

Zusammen mit Abramson und Teasdale hat Seligman (1978) diese Theorie noch um attributionstheoretische Überlegungen erweitert. Damit wurde die Ursachenzuschreibung nicht länger in Abhängigkeit zu unkontrollierbaren Ereignissen verstanden, sondern viel mehr in Abhängigkeit zur Wahrnehmung der Situation durch den Menschen.

Die jeweils getroffene Kausalitätszuschreibung, unabhängig davon ob es sich dabei um eine realistische oder unrealistische handelt, kann sich auf die Bewertung anderer Situationen auswirken und damit das Hilflosigkeitsgefühl verallgemeinern, je nachdem wie wiederum die Hilflosigkeit bewertet wird (etwa als stabil/instabil, internal/external). SELIGMAN (1975) leitet ab, dass sich damit eine Hilflosigkeitserwartung ausprägen kann.

ABRAMSON et al.(1978) konnten eine Verbindung zwischen erlebter Hilflosigkeit (und dem resultierenden Kontrollverlust) der Bewertung zukünftiger Situationen über Attribution belegen.

Im Zentrum der sozial-kognitiven Theorie nach BANDURA (1977) steht das Konzept der Selbstwirksamkeitserwartung. BANDURA (1981) definiert diese Selbstwirksamkeitserwartung als die Überzeugung einer Person mit bestimmten Fähigkeiten und Fertigkeiten ausgestattet zu sein, die einen positiven Umgagn mit bestimmten Anforderungen ermöglichen, womit die Selbstwirksamkeitserwartung als Teil des organisierten Wissens einer Person um sich selbst zu verstehen ist.

BANDURA differenziert zwischen der Ergebniserwartung und der Selbstwirksamkeitserwartung. Erstere beschreibt dabei die Erwartung, dass ein bestimmtes Verhalten zu einem gewissen Ergebnis führen wird, die letztere hingegen umfasst die Erwartung, die erforderlichen Fähigkeiten und Fertigkeiten inne zu halten, die es ermöglichen, das in der Ergebniserwartung beschriebene Verhalten auch in die Tat umsetzen zu können.

Entscheidend ist dabei, dass die Bewertung der eigenen Fähigkeiten bzw. der Glaube an das Vorhandensein der nötigen Fähigkeiten und Fertigkeiten bedeutsam ist, und nicht, ob diese Bewertung tatsächlich realistisch, also zutreffend ist. 
Weiter lässt sich zur Selbstwirksamkeitsüberzeugung festhalten, dass diese in Handlungssituationen bestimmt, wie Fähigkeiten und Fertigkeiten umgesetzt werden, dass sie in Zusammenhang mit der Entwicklung der sozialen Kognition steht und sowohl spezifische, wie auch allgemeine selbstbezogene Überzeugungen beinhaltet.

Aus dieser Sicht heraus bezeichnet BANDURA die Selbstwirksamkeitsüberzeugung als „kognitive[n] Bestandteil eines Reaktionssystems“ (BANDURA 1988), welches sich zu einem allgemeinen Kompetenzgefühl entwickeln kann, sofern es in mehrfacher anforderungsspezifischer Form in einem Bereich vorhanden ist. Einfacher ausgedrückt kann sich die Überzeugung, über eine Vielzahl von Fähigketien und Fertigkeiten zu verfügen, zu einem Gefühl der Kompetenz verbinden.

Die Selbstwirksamkeitserwartungen können als Ressource zur Stressbewältigung dienen, die zum einen die Einschätzung einer Stresssituation positiv verändern können und zum anderen die Bewältigung der Situation direkt beeinflussen können.

JERUSALEM und SCHWARZER (1992) haben belegt, dass eine hohe Selbstwirksamkeitserwartungen dazu führt, dass Menschen an sie gestellte Anforderungen eher als Herausforderung wahrnehmen, wohingegen Menschen mit niedriger Selbstwirksamkeitserwartung auf Grund der Unsicherheit bezüglich der eigenen Handlungsmöglichkeiten eher zu einer Bewertung von Anforderungen als Bedrohungen oder Verluste neigen.

Wenn eine Person also in der Lage ist, Einzelerfahrungen miteinander in Kontext zu setzen und in ihr Selbstsystem zu integrieren, so ist die Voraussetzung für die Herausbildung einer stabilen Selbstwirksamkeitserwartung gegeben.

Wenn nun noch die Ergebniserwartung und die Selbstwirksamkeitserwartung deckungsgleich sind, wenn also die Erwartung, dass ein bestimmtes Verhalten, zu welchem man in der Lage ist, zu einem bestimmten (erstrebenswerten) Ergebnis führt komplett erfüllt wird, so kann die Person Vertrauen in ihre Kompetenz entwickeln. Das führt dazu, dass sich das Verhalten und das psychische Befinden der Person verändern können. 


\section{Erziehung in der Migration als weiterer Aspekt des Akkulturationsstresses}

Um an dieser Stelle nochmals einen knappen Überblick über verschiedene Zusammenhänge der in dieser Arbeit untersuchten Thematik bieten zu können, folgt eine knappe Rekapitulation der bereits beschriebenen Erkenntnisse:

Bisher wurde erklärt, welche praktische Implikationen sich aus einer (nationalstaatliche) Grenzen überschreitenden Migration ergeben, wobei insbesondere die Integration und Akkulturation in eine fremde Gesellschaft erhebliche Herausforderungen an die Migranten (und in gewisser Weise auch an die Aufnahmegesellschaft) stellen.

Im weiteren Verlauf der Arbeit wurde darauf hingewiesen, dass sich aus der Migration besondere Anforderungssituationen für die Migranten ergeben, wobei auch betont wurde, dass diese nicht nur für Migranten der ersten Generation gelten, sondern sich auch auf die nachfolgenden Generationen übertragen können.

Weiterhin wurde darauf verrwiesen, dass diese Anforderungen in der Wahrnehmung der Migranten häufig die eigenen Ressourcen übersteigen, was, wie in Kapitel 4 gezeigt wurde, zu Stress führt.

Der Begriff des Akkulturationsstress und seine besondere Bedeutung wurden ebenso beleuchtet.

Einen hervorragenden Überblick zu den Verknüpfungen der bisher genannten Elemente liefert UsLUCAN $(2010)^{61}$. So betont er, dass die Situation vieler türkischer Gastarbeiter in gewisser Hinsicht einige Besonderheiten aufweist, da hier nicht "nur" eine Migration von einem Land in ein anderes erfolgte, sondern zusätzlich die Unterschiedlichkeit dieser beiden Länder sehr stark ausgeprägt ist.

Wie in dieser Arbeit mit dem Verweis auf BROOKER angedeutet, konstatiert auch USLUCAN, dass der unterschiedliche Grad an Modernität (der Türkei und Deutschlands) sich auf den aus der Eingliederung in die neue Umgebung resultierenden Stress verstärkend auswirkt. Nochmals erhöht wird dieser verstärkende Effekt, wenn die Migration ihren Ausgangspunkt in einer eher rural charakterisierten Region hatte. Hier könnte dann tatsächlich davon gesprochen werden, dass die Globalisierung dazu geführt hat, dass das Erleben von Migration gesteigert wurde, da letztlich auch die Grenzen zwischen der sogenannten Ersten

\footnotetext{
${ }^{61}$ UsLUCAN, HH., (2010), Professor an der Universität Potsdam, unveröff. Skripte und
} Vortragstexte freundlicherweise zur Verfügung gestellt. 
Welt und der Dritten Welt überschritten wurden und damit die Grenzen zwischen prämodernen und postmodernen Gesellschaften (vgl. BROOKER 2003). USLUCAN beruft sich auf BERRY wenn er festhält, dass „[...] der Akkulturationsstress, d.h. der Stress, der bei der Begegnung mit einer neuen Kultur auftritt, dort stärker [ist], wo die Diskrepanzen zwischen Herkunft- und Aufnahmekultur groß sind [...]" (2010).

USLUCAN stellt des Weiteren die Behauptung auf, dass die Wanderung von einer islamischen Gesellschaft hinein in die pluralistische Gesellschaft Deutschlands eine Besonderheit darstellt, insofern als dass die gesamte Familie zur Anpassung und Erweiterung ihrer Verhaltensweisen gedrängt wird. Dies stellt einen besondereren Druck hin zum Erlernen neuer Standards, wodurch "in der Regel auch eine Entfernung von den Werten der Herkunftskultur" statt findet.

Weiter behauptet USLUCAN, dass besonders islamische Migrantenfamilien unter den widersprüchlichen Wünschen, Neues in die Persönlichkeit zu integrieren und dabei aber auch die Werte der Herkunftskultur zu bewahren, leiden, nicht zuletzt weil der oben erwähnte zusätzliche Stress, der sich durch die Belastungen der Migration als auch des Konflikts zwischen Moderne und Religiosität ergibt, sie strapaziert.

USULCAN führt weiter aus, dass es unabhängig davon, ob im Zuge der Migration und der Auseinandersetzung mit der neuen Situation eine Präferenz in Richtung der Aufnahmen neuer Werte, oder einer Rückbesinnung auf die Herkunftskultur vorliegt, eine Veränderung der Werte zu erwarten ist, da auch bei der Beibehaltung der „alten“ Werte eine Veränderung stattfindet.

Es ist häufig zu beobachten, dass beim Erhalten der Werte der Herkunftskultur eine eher defensive Haltung gegenüber der neuen Umwelt zu Tage tritt der Erhalt der mitgebrachten Werte verstärkt die Abgrenzung zur aufnehmenden Kultur.

Auch die Erziehung der Kinder in der Fremde stellt (gerade die erste Generation der) Auswanderer vor zum Teil erhebliche Probleme, da auch in diesem Kontext die Frage der Werte, die an den Nachwuchs weitervermittelt werden sollen, auftaucht.

Somit könnte man also sagen, dass nicht nur die zweite Sozialisation der Auswanderer, sondern auch die (erste) Sozialisation der Kinder durch die Wanderung erschwert wird. 
UsULCAN merkt hier nochmals an, dass insbesondere im Falle von Rückzugstendenzen zur Herkunftskultur besondere Effekte auftreten. Er führt jedoch auch aus, dass die Effekte, die besonders bei einer religiösen Orientierund der Eltern auftreten, durchaus ambivalent ausfallen.

Zunächst können die jungen Lerner (die Kinder der Migrierten) durch die religiöse Sozialisation ein besseres Verständnis für die Lebenswelt ihrer Eltern erlangen, was Hand in Hand mit einer Rückbindung an die elterliche Herkunftswirkung stattfinden kann. Dies wiederum schafft Möglichkeiten zur Reflektion der eigenen Tradtionen sowie einen intellektuellen Auseinandersetzung mit ihr.

Eine solche islamische Erziehung in der Fremde, so darf nach UsULCAN angenommen werden, ist eher reflektiert, in dem Sinne, dass im fremden Umfeld eine bewusstere Auseinandersetzung mit der Religion statt finden muss, da der gesellschaftliche Rückhalt, der in einer islamischen Gesellschaft zu erwarten ist und der dort häufig zur unreflektierten Übernahme der religiösen Werte als Alltagsgewissheit führt, nicht (in gleichem Maße) vorliegt.

Auch kann die türksich-islamische Erziehung im Aufnahmeland als der Tendenz nach eher behütend und kontrollierend beschrieben werden.

Diese Erziehung, die über die Vermittelung von Werten wie Loyalität und Gehorsam aber auch durch die Betonung der Bedeutung der Familie durchaus einen festeren Zusammenhalt im familiären Verbund generieren kann, kann aber ein Hemmnis für die Anpassung an die Aufnahmegesellschaft für die heranwachsende Generation darstellen.

So zeigt sich hier also eine Ambivalenz, da einerseits die Familie als Ressource wirken kann, die die soziale Isolation außerhalb der Familie abdämpft, andererseits kann dieser Zusammenhalt aber auch als Integrationshemmnis wirken.

Die Betonung der kulturellen Werte der Eltern in der Erziehung der Kinder kann nach NAUCK und ÖZEL (1986) als eine Reaktion auf die sich als bedrohlich darstellende Migrationssituation interpretiert werden. Die vermeintliche Entfremdung der Kinder von den Eltern wird von den Eltern darauf zurückgeführt, dass die Akkulturation mit größerer Geschwindigkeit als die der Eltern von statten geht.

Die Erziehungsinhalte, die in der Herkunftskultur der Eltern von besonderer 
Bedeutung sind, lassen sich vor allem als das Vermittlen von Höflichkeit und guten Manieren beschreiben (etwa das Beherrschen der Eigenheiten der Gastfreundschaft oder das korrekte Ansprechen der älteren Geschwister etc.).

LEYENDECKER (2003) betont jedoch, dass die fast archaisch anmutenden Werte wie Respekt, unbedingter Gehorsam und strenge Hierarchie keine spezifisch islamisch oder türkischen Werte darstellen, sondern vielmehr eine häufig anzutreffende Eigenheit von kollektivistischen Kulturen, die aber durchaus noch einen gewissen Nutzen für den individuellen Beteiligten haben. Der Nutzen ist vor allem darin gegeben, wenn ein gewisses Gleichgewicht erzielt wird, zum Beispiel indem unbedingter Gehorsam im Gegenzug mit Fürsorge oder Hilfe in Notsituationen beantwortet.

Bei UsULCAN finden sich auch Ausführungen darüber, wie sich die islamische Erziehung im Verlauf des Lebens der Kinder verändert. Er beschreibt im Wesentlichen drei verschiedene Phasen:

Zunächst eine Phase mit permissivem Charakter, die in etwa bis zum Alter von sieben Jahren anhält (wobei gerade in den ersten drei Lebensjahren vor allem die Mutter für ein Gefühl von Geborgenheit und Wärme verantwortlich ist).

In der sich anschließenden Phase, die etwa bis ins 14. oder 15. Lebensjahr reicht, findet eine deutlicher Wandel hin zu einer deutlich autoritären erzieherischen Haltung statt. Des Weiteren ist diese Phase dadurch charakterisiert, dass die Kinder getrennt vom gleichgeschlechtlichen Elternteil erzogen werden.

Ab der einsetzenden Pubertät wird es zusätzlich üblich, mehr Überwachung über das Kind zu haben und damit sicher zu stellen, dass guter Umgang des Kindes gegenüber Anderen gepflegt wird. Mit dieser Art der Überwachung soll das Kind vor allem vor nachstehenden Folgen geschützt werden. Dass diese Art der Überwachung in direktem Zusammenhang mit Akkulturationsstress stehen könnte ist den Eltern selten bis gar nicht klar:

Not surprisingly, acculturative stress has been linked to academic performance and negative mental health consequences. For example, acculturative stress has been positively associated with lower academic performance, lower quality of life in general, excessive alcohol intake, body dissatisfaction and bulimia, identity confusion, anxiety, psychosomatic symptoms, depression, and suicidal ideation. Moreover, during the past five years, research related to acculturative stress has begun to identify variables that might mediate or moderate the relationship between acculturative stress and depression. [...] Similarly, perceived social support from friends has been shown to have a buffering effect 
between acculturative stress and depression for Korean adolescents from immigrant families in the United States. Likewise, family closeness, hopefulness for the future, and financial resources have been found to provide a buffer against the negative effects of acculturative stress experienced by Mexican immigrants. Thus, the relationship between acculturative stress and mental health in not necessarily a simple linear relationship but can be buffered by a range of personality variables, social support, and financial resources. (Heppner, Park, Wei 2006:18)

HePPNeR, PARK und WeI verweisen darauf, dass Akkulturationsstress unter anderem in Zusammenhang mit schlechterer akademischer Leistung, geringerer Lebensqualität im Allgemeinen, exzessivem Alkoholkonsum, Körperunzufriedenheit und Bulimie, Identitätsstörungen, Angstzuständen, psychosomatischen Symptomen, Depressionen und Suizidgedanken gebracht werden kann. Darüber hinaus hat die Forschung laut HEPPNER ET AL. auch verschiedene Variablen entdeckt, welche möglicherweise die Verbindung zwischen Akkulturationsstress und Depressionen moderieren oder mediieren. So konnte etwa eine dämpfende Wirkung von wahrgenommener sozialer Unterstützung auf den Zusammenhang zwischen Akkulturationsstress und Depressionen festgestellt werden. Des Weiteren verweisen HEPPNER ET AL. auf Studien, die gezeigt haben, dass die negativen Auswirkungen von Akkulturationsstress auch durch familiäre Nähe, einer positiven Zukunftssicht und finanzielle Ressourcen gedämpft werden können. So kommen die Autoren zu dem Schluss, dass der Zusammenhang zwischen Akkulturationsstress und mentaler Gesundheit nicht notwendigerweise einfach linear sein muss, da eine Reihe persönlicher Variablen eine dämpfende Wirkung haben können.

Jüngst erschien von ANDREAS ZICK (2010) ein Werk, dass in einigen Punkten dem entspricht, was diese Arbeit nachzuweisen und anzureißen sucht. Es bietet einen hervorragenden Überblick über den gegenwärtigen Forschungsstand hinsichtlich Akkulturationsstress, das hier umfangreich zur Übersicht zitiert werden soll:

Die Borderland Identity Theory von Lavie und Swedenburg (1996) ist eine relativ kleine, anthropologisch orientiert Theorie, die sich mit Identitätszuständen von Immigranten beschäftigt. Sie offeriert die These, dass Immigranten so genannte Borderland Identities ausbilden können, also Identitäten, die zwischen der Herkunftskultur und der neuen Kultur, der sie begegnen liegen. Psychologisch kann man sie als bidimensionale Identitäten verstehen (vgl. Kap. 4.2.4). Eine weitere Annahme ist, dass dies mit der ständigen Gefahr einhergeht, in Fallen zu treten sowie mit der größeren Wahrscheinlichkeit, in Zonen der Gewalt zu geraten; also im sozialpsychologischen Sinne in interkulturelle Konflikte zu geraten, die bis zur Gewalt reichen können. Meines Erachtens ist nicht nur die These sehr weit reichend und daher unpräzise, sondern auch schwach begründet. Empirisch wird sie 
auch nur durch wenige Hinweise gestützt. Das heißt nicht, dass sie nicht ernst zu nehmen ist und Neuankömmlinge allein deshalb weil sie neu sind und damit sub-dominant, Adressanten von Vorurteilen etc. sind, also mit einer höheren Wahrscheinlichkeit intergruppalen Konflikten und Gewalt ausgesetzt sein können als Einheimische. Die beiden Thesen werden hier auch deshalb aufgeführt, weil sie relativ häufig so oder ähnlich in einigen Theorien genannt werden und das Konstrukt der Grenz-Identität häufiger zitiert wird. (379f)

Bathia und Ram (2001) stellen die kritische Frage, wer diesen Zustand der Borderland Identität feststellt? Wie weiß jemand, ob er/sie integriert ist, oder sich in einer Grenzsituation befindet? Das ist eine entscheidende Frage für die Akkulturationsforschung. Sie nehmen an, dass die Bedeutung der Selbst-Anderer-Beziehung durch die Beteiligung an alltäglichen soziokulturellen Praktiken und die sozialen Beziehungen, die in die Praktiken eingebettet sind, mediiert, strukturiert und organisiert wird. Sie wählen zur Analyse einen diskursiven Ansatz, der die Konstruktion von Selbst und Identität fokussiert. Dabei richten sie ihr Augenmerk auf die Frage wie post-koloniale Praktiken und Politiken in die Alltagserfahrungen von heutigen Migranten verwoben sind. Sie verweisen darauf, dass vor allem Migranten aus der sogenannten Dritten Welt postkoloniale Erfahrungen haben, die den Akkulturationsprozess beeinflussen. Der besondere Blick der Analyse ist dabei auf die Beziehung zwischen den Konstruktionen von Rasse, Macht und Status der Migranten vor und nach der Migration gerichtet. Bathia und Ram postulieren, dass die Gemeinschaft und Identität der Einwanderer aus den ehemaligen Kolonien durch "Geschichten über Diskriminierung, Verfolgung und Ausbeutung" aufrechterhalten werden. Migranten lebten durch den Erhalt ihrer Herkunftskultur wie einer Diaspora (Tölöyan, 1996). Bathia und Ram rekurrieren dabei auf das skizzierte Konzept der Borderland Identity von Lavie und Swedenburg (1996). Sie verweisen darauf, dass diese Grenzland-Identität durch die „ständige Gefahr, in die Minen zu treten, in Zonen der Gewalt zu geraten“ gekennzeichnet ist.

Bathia und Ram (2001) verweisen in heftiger Kritik an Berry (1997) ebenfalls darauf, dass dieser die Themen Konflikt, Macht und Asymmetrie der sozialen Beziehung zwischen Allochtonen und Autochtonen nicht behandelt. Sie meinen, dass Akkulturation ein Prozess des Moving and Mixing zwischen der Kultur der autochtonen und allochtonen Gruppen ist. Akkulturation wird daher als eine Geschichte hybrider Identitäten verstanden. Die Theoretiker schauen auf fundamentale Machtdifferenzen zwischen dominanter Aufnahmegesellschaft und einwandernder Minderheit. Diese wird verbunden mit Konzepten der Identitätspolitik und der Anstrengung der nicht-dominanten Gruppen ihren Opferstatus zu bewältigen. Insgesamt erscheinen allerdings die Geschichten und ihre Interpretationen anekdotisch, zumal das Verfahren der Auswertung nicht explizit begründet wird.

Dieser [Prozess der Ethnisierung - Ergänzung I.Ö.] verlaufe in drei Stufen. Auf der ersten Stufe verlören Einwanderer ihre Gesllschaftlichkeit. Sie würden von der autochtonen Mehrheit zu Nicht-Gesellschaftsmitgliedern - eben „Ausländern“ - erklärt. Auf der zweiten Stufe begegneten dann die Einwanderer besonderen Grenzen und Umgangsformen, die sie von der Mehrheit abgrenzten. Auf der dritten Stufe erfolge die Minorisierung. Die Einwanderer würden in die Rolle von Mitgliedern einer ethnischen Minderheit gedrängt. Bukow und Llaryora argumentieren auf der Grundlage eines Labeling-Ansatzes und stellen fest, dass die Ethnisierung letztendlich eine besondere Form der Etikettierung ist. Auf die Etiketten griffen Einwanderer zurück, um ihre Identität $z u$ sichern. Die ethnische Identifikation bliebe als Rückzugsgrundlage für die starken Überlastungen. Das heißt, der Rückgriff von Einwanderern auf die ethnische Identität entstehe in Wechselwirkung zwischen ihrer Gruppe und der autochtonen Mehrheit und sei eine Re-Definition als Reaktion auf den Prozess der Ethnisierung seitens der Einheimischen. Dabei sei der Ausgangspunkt der Ethnisierung immer die Politik. (387)

\section{Auf anderer Ebene schlussfolgern PANKSEPP ET AL:}

[...] Accordingly, affect could not exist independent of consciousness, since in essence it is something that exists as part and parcel of conscious perception. Even in the absence of complex intellectual function, affective experience (an apparently intrinsic brain function) 
appears to exert a powerful influence on behavior in all mammals, and new models have been developed which allow such issues to be addressed objectively across species. (2002: 113f)

Die Autoren konstatieren, dass Affekt und Bewusstsein nicht unabhägig voneinander existieren können, da sie im Grunde beide Bestandteile der bewussten Wahrnehmung sind. Selbst wenn keine komplexen intellektuellen Funktionen vorhanden sind, üben affektive Erfahrungen einen bedeutsamen Einfluss auf das Verhalten aller Säugetiere aus. PANKSEPP ET AL. betrachten diese Eigenschaft als intrinsische Gehirnfunktion. Daher wurden neuere Modelle entwickelt, um derartige Beobachtungen objektiv über Speziesgrenzen hinaus vollziehen zu können.

[...]

\{In conclusion, lessons from the history of science suggest the following:

You must build a field of scientific inquiry using the robust tools that already exist in related disciplines and from such an approach, emergent, testable ideas can be expected;

If you are willing to embrace evolutionary theory and the Medical Model of disease, then there must be a genetic basis to many fundamental aspects of human behavior;

Many of the basic constituents of human behavior are likely to be present in animals other than humans (while at the same time not denying that many additional mental potentials arose from more recent cortico-cognitive developments); and\}

As such, the use of animal models to study human behavior using the tools of chemistry, physics, biology, biochemistry, and molecular biology can be fruitfully undertaken.

With the development of new molecular techniques and the subsequent collection of new data sets in model species, a more thorough understanding of the genetically promoted characteristics of human mind and behavior will gradually emerge. This, in no way, denies the special neuro-symbolic and cultural capacities that have emerged which allow humans to have thoughts about their thoughts and feelings that seem beyond the ken of practically all other species. (ebd.)

PANKSEPP ET AL. fassen zusammen, dass Modelle des Verhaltens von Tieren unter Zuhilfenahme der Instrumente der Chemie, Physik, Biologie, Biochemie und Molekularbiologie durchaus fruchtbare Ergebnisse im Bereich des Studiums des menschlichen Verhaltens erbringen können. Sie prognostizieren, dass mit der Entwicklung neuer molekularer Techniken und der daraus resultierenden Sammlung neuer Datensätze für verschiedene Modellspezies ein tieferes Verständnis der genetisch bevorzugten Charakteristika des menschlichen Verstandes und Verhaltens gewonnen werden wird.

Die Autoren betonen dabei, dass dies in keinem Widerspruch zu den besonderen neuro-symbolischen und kulturellen Fähigkeiten steht, welche sich herausgebildet haben und dem Menschen ermöglichen, Gedanken über eigene 
Gedanken und Gefühle zu haben, die den Horizont jeder anderen Spezies übertreffen.

So argumentieren PANKSEPP ET AL. abschließend, dass mit der Schöpfung solider neuropsychologischer Grundlagen auch passendere intellektuelle Räume für ein wissenschaftliches Verständnis von selbst fast unmerklich entwickelten Funktionen des menschlichen Verstandes geschaffen werden könnte.

Zu guter Letzt liefern WONG und WONG (2006) aus dem Handbuch der multikulturellen Perspektiven von Stress und Coping-Verfahren folgende Studie zur Emotionsregulierung im interkulturellen Kontext:

\section{Comparing and Contrasting Adaptation and Adjustment}

In our work we have found that it is important to make a distinction between adaptation and adjustment. Using Ward's approach, we believe that adaptation is based in the sociocultural domain (Ward, 2001), that is, it is the process of altering one's behavior to fit in with a changed environment or circumstances, or as a response to social pressure. One of the most well known models of adaptation, for instance, is Berry's (1994) analysis of the interaction styles for sojourners, immigrants, and refugees. In this model, four categories of interaction style are identified on the basis of the yes/no answers to two questions: (1) is it important to maintain my cultural identity and characteristics, and (2) do I value and want to maintain relationships with people of the host culture? Individuals who say yes to both are considered "integrators;" those who say no to both are "marginalizers." Those who say yes to the first and no to the second are "separators,"while those who say no to the first and yes to the second are "assimilators." clearly these processes refer to behavioral changes made in response to different environments.

In contrast, we define adjustment as the subjective experiences that are associated with and result from attempts at adaptation, and that also motivate further adaptation.

Adjustment, therefore, essentially refers to the general concept of well-being, which is an affective evaluation of one's life situation. In line with this definition, previous researchers have incorporated a wide range of outcome measures of adjustment, including selfawareness and self-esteem (Kamal und Maruyama, 1990), mood states (Stone Feinstein und Ward, 1990), and health status (Babiker, Cox, und Miller, 1980) (all cited in Ward, 2001). Other typical measures of adjustment involve other subjective experiential constructs that refer to well-being, such as anxiety, mood, depression, subjective wellbeing, satisfaction, and happiness. (Wong und Wong 2006: 387 f)

Emotion regulation allows individuals to engage in clear thinking about intercultural incidents without retreating into psychological defenses. If sojourners do not have the ability to regulate or control their emotions, they will be unlikely to adjust well because they will be locked into their automatic or habitual ways of thinking and interacting with the world. Emotion regulation seems likely to be the gatekeeper skill for intercultural adjustment. (WONG und WONG 2006: 390)

Zusammenfassend lässt sich über Akkulturationsstress also sagen, dass Ausgrenzungserfahrungen zu Migrationsstress führen und dadurch oder auch schon vorher ein problematisches Verhältnis der Individuen zu sich selbst entsteht. Als Folge oder Teilaspekt des Akkulturationsstresses nehmen die Migranten ein Unerwünschtheitsgefühl und Minderwertigkeitsgefühl in sich wahr. Das 
problematische Verhältnis, das die Individuen zu sich selbst entwickeln können, ist nicht zwangsweise bewusst, auch wenn sie das Gefühl wahrnehmen, ohne es beschreiben zu können.

\section{Finale Überlegungen und Belege}

\section{Akkulturationsstress als mögliche Lernblockade}

Nachdem die individuellen Folgen von Stress ebenso dargelegt wurden wie die sozialen Folgen, wurde der Akkulturationsstress als solcher nochmals ausgeführt. Wie bereits im Kapitel 3 angedeutet, werden im Folgenden Verknüpfungen zwischen (Akkulturations)Stress und der Fähigkeit zu Lernen hergestellt, bevor diese belegt werden.

Generell lässt sich sagen, dass sich Akkulturationsstress derartig blockierend auswirkt, dass ein formelles Lernen nicht möglich ist und somit andere Möglichkeiten des Lernens eröffnet werden müssen. Das kann beispielsweise das informelle Lernen in der sicheren Gruppe/Herde und daher das Lernen unter angenehmen Bedingungen sein. Ein Beleg dafür ist die Neurodidaktik mit HüTHERS Atmosphären-Beschreibung. Gerade Migranten benötigen eine spezielle kultursensible Atmosphäre, wie die Literaturübersicht in der Einführung gezeigt hat sind bisher allerdings keine nutzbaren Studien zu der Lücke 'Migration und Lernen' vorhanden. Daher beabsichtigt diese Arbeit nun die ersten Schritte in die Richtung zu gehen, diese genannte Lücke zu füllen und dabei vor allem den neurobiologischen Hintergrund zu berücksichtigen. Wie zuvor dargelegt, mangelt es in den meisten Lernsituationen bisher an der 'Herde' und der 'sicheren Umgebung'. Der Stresspegel im Sinne des Drucks ist zum Beispiel in Integrationskursen zu hoch und katalysiert keine intrinsische Motivation, sondern erschwert im schlimmsten Fall den Zugriff auf das vorhandene Gedächtnis und Wissen. Neues kann in diesem Kontext noch schwerer gelernt werden und schließlich können all diese Unsicherheitsfaktoren die Panik- und Furchtsysteme aktiveren und somit regressive Verhaltensweisen und körperliche Symptome hervorrufen. 


\section{Belege}

Im Folgenden finden sich versuchsweise einige Belege dafür, dass Migrationsstress Lernen blockiert, sich körperlich auswirkt und ohne Herdenverhalten schwer von den Individuen zu tragen ist.

\subsection{Fragebogenerhebung}

Zur Erfassung der Lebenszufriedenheit alter Migranten wurde im Rahmen dieser Arbeit eine orientierende Untersuchung durchgeführt. Dabei kam neben einem allgemeinen Fragebogen zu sozialen Eckdaten der Personen der Fragebogen zur Lebenszufriedenheit (FLZ, siehe Anhang) von HEINRICH und HERSCHBACH (1996) zum Einsatz. Dieser wurde den Teilnehmenden der Befragung in einer türkischen Version vorgelegt (FLZ türkische Version, siehe Anhang), deren Gültigkeit mittels Expertenvalidierung zuvor festgestellt wurde.

\section{Methode}

Ohne Anspruch auf ein experimentelles Forschingsdesign wurde online in sozialen Netzwerken (StudiVZ und Facebook) zur Teilnahme an der Erhebung aufgerufen. Es wurden in Deutschland lebende Migranten türkischer Herkunft für eine Befragung mittels Fragebogen, das bei der Auswertung anonymisiert werden sollte, gesucht. Teils meldeten sich Freiwillige direkt oder mittelbar über ihre Kinder, die diesen Aufruf gelesen hatten und ihre Eltern / Großeltern zur Teilnahme motivierten.

Die Fragebögen (FLZ und Fragebogen zu Sozialdaten) wurden mit einem frankierten Rückumschlag zum Teil als Einzelexemplare, zum Teil in einer Menge von bis zu 8 Stück versandt. Manche Teilnehmer haben selbstständig die Fragebögen verfielfältigt und mehr zurückgesandt, als sie empfangen hatten.

\section{Resultate}

Der Rücklauf betrug insgesamt 67 Fragebögen, wobei 2 aus Bielefeld, 2 aus Bremen, 15 aus Göttingen, 8 aus Hann. Münden, 4 aus Heilbronn, 10 aus Kassel (bzw. Witzenhausen), 11 aus Northeim und 9 aus Schweinfurt stammten. Bei 6 Fragebögen konnte keine Zuordnung zu einer Stadt erfolgen. Im Anhang finden sich die hier dargestellten Ergebnisse in tabellarischer Form. 


\section{Soziale Eckdaten}

Von den empfangenen Fragebögen stammen 32 von weiblichen und 35 von männlichen Teilnehmern, wobei 46 eine türkische, 16 eine deutsche und 5 Personen eine andere Nationalität angaben. Der Mittelwert des Alters ist 55,91 Jahre, der Median 55 Jahre (Altersminimum 38, Altersmaximum 78 Jahre).

Die meisten der Teilnehmer sind verheiratet (57 Pers.), 3 sind ledig, 5 verwitwet und jeweils eine Person ist geschieden oder getrennt lebend.

Der Mittelwert des Aufenthaltes in Deutschland für diese Personen beträgt 34,02 Jahre bei einem Spektrum von minimal sieben Jahren bis 48 Jahren.

Bei 13 Personen fehlte die Angabe nach der Anzahl der Arbeitsjahre, der Mittelwert der Arbeitsjahre der übrigen 54 Personen betrug 21,87 Jahre (Min. 0, Max. 44Jahre).

Bei der offenen Beantwortung der Frage nach dem Beruf gaben 19 von den 67 Personen an, Arbeiter zu sein (in türkischer Sprache), 13 machten keine Angabe, 4 gaben Bauarbeit und 4 gaben Rentner(-in) als Beruf an. Zwei Personen gaben den Beruf in Deutscher Sprache an („Metal Arbeiter“ und „Töpfer“).

Der Mittelwert der von 65 Personen angegebenen Kinderzahl betrug 2,69 (Min. 1, Max. 9), wovon 38,8\% zwei, 31,3\% drei und 11,9\% vier Kinder haben.

Die Frage nach Kindern, die in Deutschland eine Berufsausbildung abgeschlossen haben, beantworteten 59 Personen. Hier wurde angegeben, dass bei $38,8 \%$ ein oder zwei Kinder eine Ausbildung abgeschlossen haben, während bei 49,3\% kein Kind eine Berufsausbildung hat.

Die Frage nach Söhnen, die in Deutschland eine Hochschulreife erreicht haben, wurde von 55 Personen beantwortet. Bei drei Personen haben 3 Söhne, bei zwei Personen ein Sohn und bei einer Person zwei Söhne ein Abitur erreicht. Bei der Frage nach Hochschulreife bei Töchtern antworteten 54 Personen, hier gaben sieben Personen an, dass eine Tochter und eine Person, dass zwei Töchter Abitur erreicht haben. 


\section{Fragebogen zur Lebenszufriedenheit}

Mithilfe des FLZ sollte bei den Probanden die subjektive Lebensqualität erfasst werden. Hierzu wurde von den drei möglichen Modulen des Fragebogens das Modul "Allgemeine Lebenszufriedenheit" mit acht Items, die jeweils von der Testperson nach "subjektiver Zufriedenheit" und zusätzlich nach "subjektiver Wichtigkeit" beurteilt werden, ausgewählt und in expertenvalidierter türkischer Form angewandt.

Der Fragebogen besteht aus einer DIN-A4-Seite, die mit einer Instruktion, fünf Fragen nach Wichtigkeit und fünf Fragen nach Zufreidenheit versehen ist. Hier soll der Proband je auf einer fünfachen Auswahlmöglichkeit jeden Aspektes (Freunde/Bekannte, Freizeit/Hobbies, Gesundheit, Einkommen/finanz. Sicherheit, Beruf/Arbeit, Wohnsituation, Familie/Kinder, Partnerschaft/Sexualität) die persönliche Wichtigkeit und die Zufriedenheit angeben. Zusätzlich wird in diesem Modul nach der globalen Lebenszufriedenheit gefragt.

Die Auswertung der Lebenszufriedenheit erfolgt mittels einer im Manual angegebenen Formel der Autoren, die dieses Modul für den Bereich BRD (Ost und West) als normiert (mit entsprechenden Normwerten) angeben. Gleichermaßen wird eine inhaltliche Validität und vorliegende Test- und Item-Kennwerte aufgeführt. 


\section{Ergebnisse eigener Erhebung / Normdaten FLZ}

In der folgenden Tabelle sind die berechneten Ergebnisse der türkischstämmigen Stichprobe im Vergleich zu den gegeben Normdaten, die aus der deutschen Population jeweils in BRD-West und BRD-Ost in den Jahren 1991 und 1994 vorgegeben sind, gegenübergestellt. Es lässt sich feststellen, dass die Werte der türkischstämmigen Stichprobe durchgängig ungefähr halb so hoch sind, wie die Werte der an Deutschen vorgegebenen Normdaten. Dies bedeutet, dass die Lebenszufriedenheit der türkischstämmigen Stichprobe (trS) in nahezu allen Aspekten nur halb so ausgeprägt ist. Die Mittelwert im Item im Beruf (berufliche Situation) ist deutlich niedriger, während der Mittelwert im Item Familienleben sich als höchster Wert der trS dem der Normdaten annähert:

Tabelle 4 Auswertungsdaten des FLZ

\begin{tabular}{|c|c|c|c|c|c|c|c|c|c|}
\hline & $\mathrm{N}$ & Min. & Max. & $\begin{array}{c}\text { Standard- } \\
\text { abweichung }\end{array}$ & $\begin{array}{c}\text { trS } \\
\text { TR_2010 } \\
\text { Mittelwert }\end{array}$ & $\begin{array}{c}\text { BRD-91- } \\
\text { West } \\
\text { Mean }\end{array}$ & $\begin{array}{c}\text { BRD-94- } \\
\text { West } \\
\text { Mean }\end{array}$ & $\begin{array}{c}\text { BRD-91- } \\
\text { Ost } \\
\text { Mean }\end{array}$ & $\begin{array}{c}\text { BRD-94- } \\
\text { Ost } \\
\text { Mean }\end{array}$ \\
\hline Freunde & 63 & $-9,00$ & 20,00 & 5,19881 & 2,86 & 7,50 & 8,33 & 7,52 & 7,05 \\
\hline Gesundheit & 62 & $-12,00$ & 20,00 & 8,86982 & 2,58 & 6,51 & 6,73 & 4,42 & 4,60 \\
\hline Hobbies & 59 & $-12,00$ & 20,00 & 5,09351 & 1,51 & 8,64 & 8,35 & 7,37 & 6,90 \\
\hline Einkommen & 60 & $-9,00$ & 20,00 & 6,67051 & 1,25 & 6,60 & 7,18 & $-0,48$ & 3,74 \\
\hline Beruf & 57 & $-12,00$ & 20,00 & 6,38107 & 0,53 & 6,35 & 6,06 & 2,03 & 3,05 \\
\hline Wohnsituation & 59 & $-12,00$ & 20,00 & 5,79445 & 2,90 & 8,74 & 8,67 & 6,51 & 6,98 \\
\hline Familienleben & 59 & $-12,00$ & 20,00 & 8,77510 & 8,22 & 10,13 & 9,80 & 11,17 & 10,00 \\
\hline Partnerschaft Sexualität & 59 & $-12,00$ & 20,00 & 8,65320 & 4,86 & 8,44 & 8,07 & 9,12 & 7,26 \\
\hline FLZ-Summenwert & 61 & $-44,00$ & 125,33 & 36,69336 & 24,84 & 62,82 & 63,21 & 47,60 & 49,22 \\
\hline
\end{tabular}




\section{FLZ Werte BRD 91/94/trS}

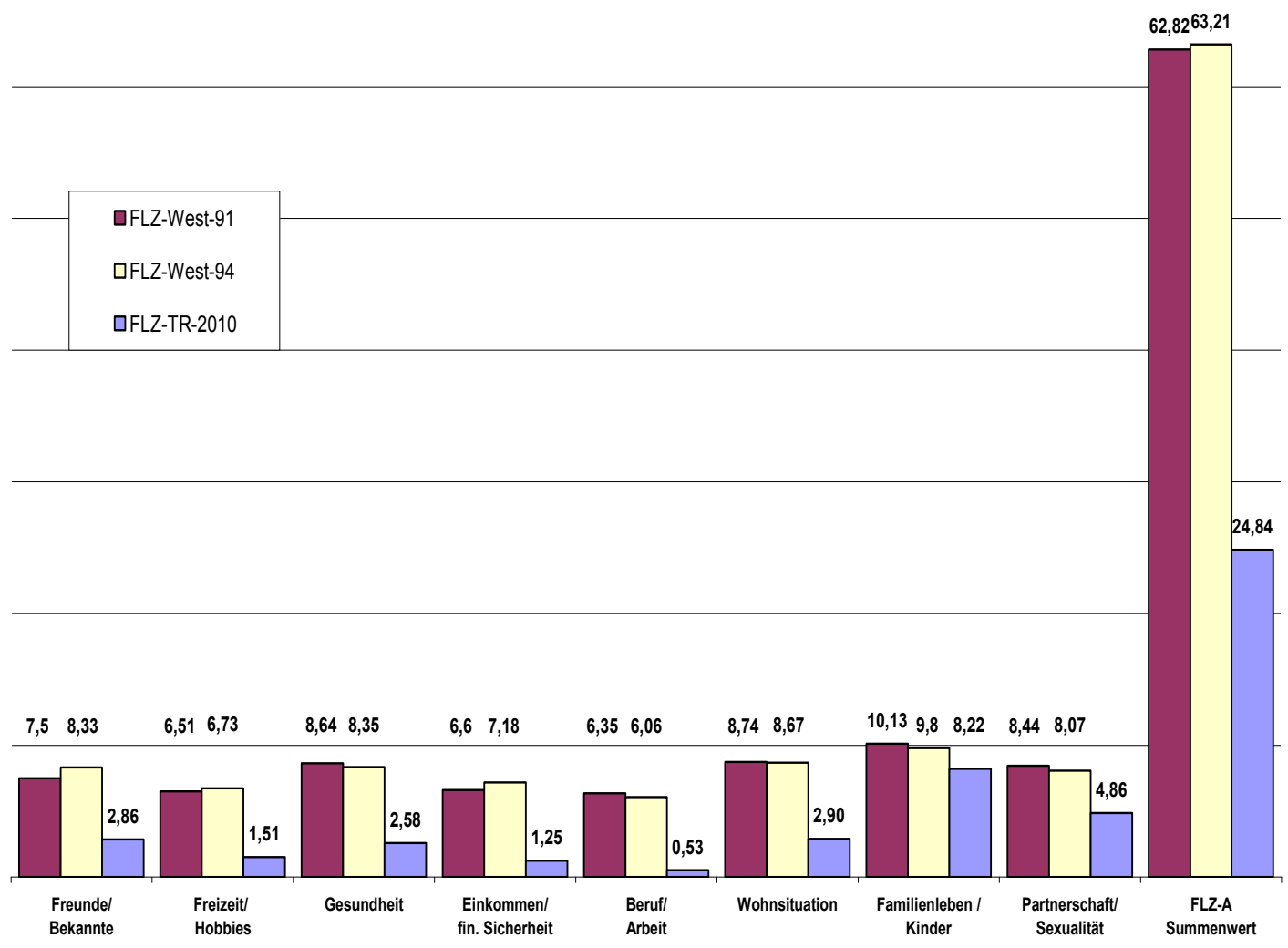

¿FLZ Werte OST 91/94/trS

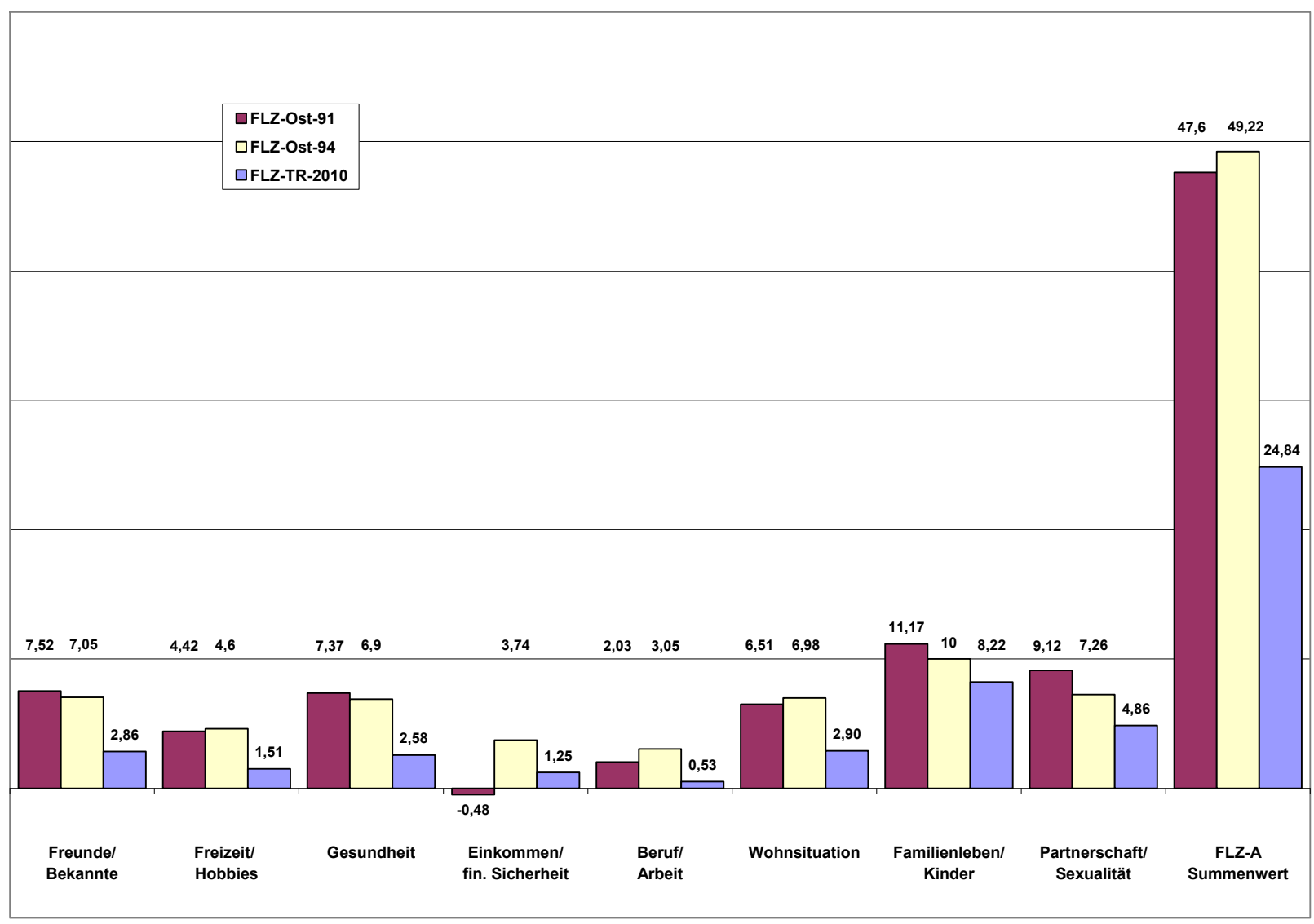




\section{Fazit der Erhebung}

Als Ergebnis dieser Erhebung kann festgestellt werden, dass die trS in den befragten Aspekten des Lebens halb so zufrieden sind, wie die deutsche Population unabhängig nach West-Ost- Unterschieden. In einem Aspekt jedoch scheint eine nahezu gleiche Zufriedenheit zu bestehen: beim Familienleben/ Kinder.

Dieser Aspekt kann durch das so genannte „Herdenverhalten“ mit Identitätsaufrechterhaltung (kollektive Identität) als mögliche Ressource, um mit dem Stress umzugehen interpretiert werden. Alle weiteren Aspekte sind niedrig, aber eine Zufriedenheit mit dem Familienleben/Kindern zu erreichen ist für viele erstrebenswert. Dabei besteht aber die Gefahr, dass, wenn dies nicht mehr gelingt, z.B. in Versorgungssituationen im Alter, die durch die Kinder nicht mehr gegeben ist, die weiteren Aspekte und die Gesamtlebenszufriedenheit noch deutlich weiter sinken können.

Dieser Interpretation der Erhebung deckt sich mit den Ergebnissen einer Studie von VON LERSNER (2011), bei welcher bikulturelle Identität und psychische Gesundheit bei Jugendlichen untersucht wurde. Das Ergebnis zeigte keine Unterschiede der Belastung bei Jugendlichen deutscher und türkischer Herkunft, während jedoch Geschlechtsunterschiede in der Belastung nachgewiesen werden konnten. Es zeigte sich eine signifikante Korrelation zwischen Akkulturationsstress und psychischen Problemen. Auch kulturelle Konflikte korrelierten positiv mit psychischen Problemen, während kulturelle Distanz keine Korrelation ergab. 


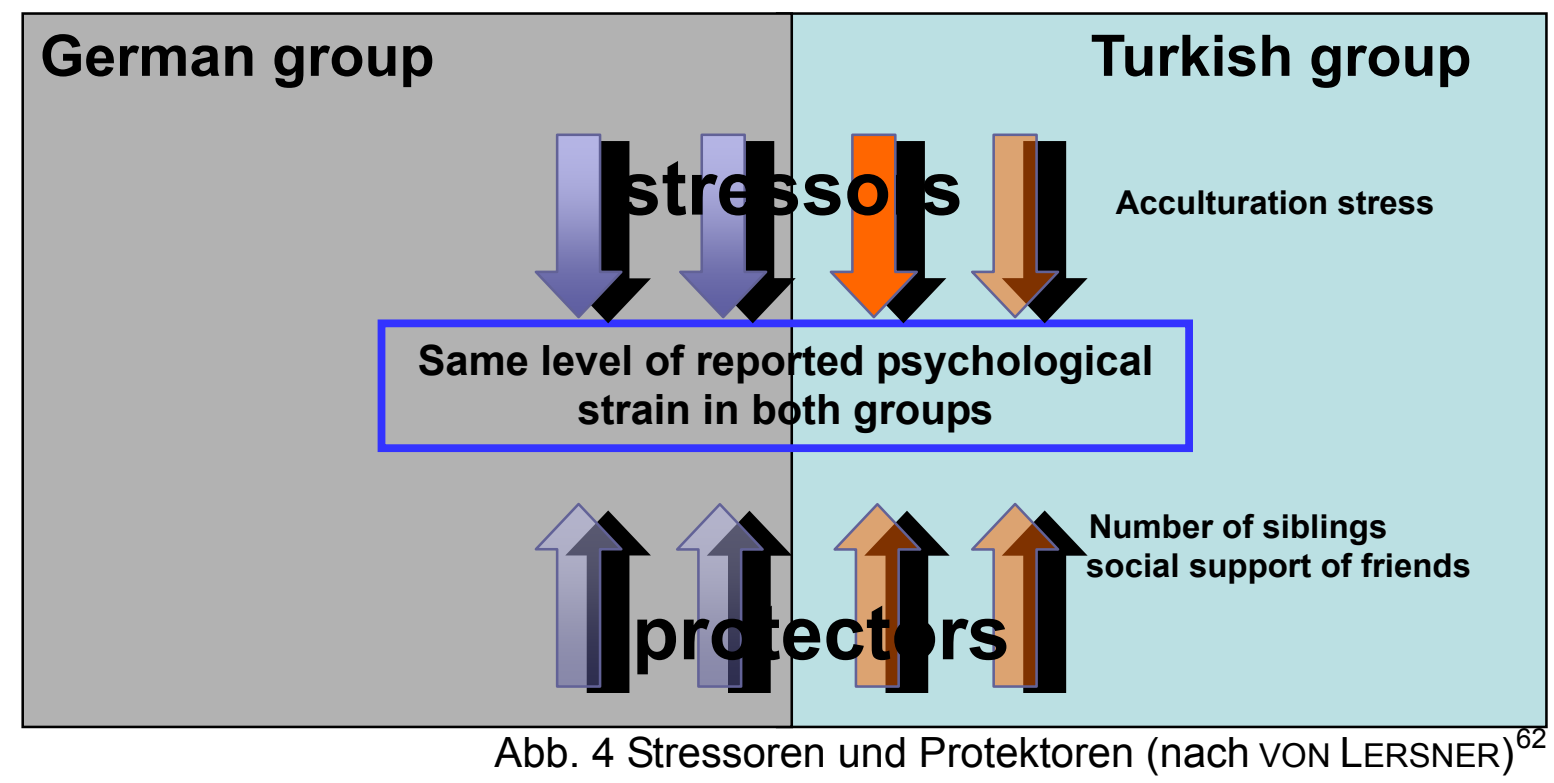

Der Akkulturationsstress wurde als Stressor bei Jugendlichen festgestellt, wobei aber Geschwister/Familie und soziale Unterstützung durch Freunde bei den türkischstämmigen protektive Faktoren bzw. Ressourcen dienend, und dementsprechend psychische Belastung relativierend, abbildeten.

\subsection{Falldarstellungen aus der psychotherapeutischen Praxis}

\section{Falldarstellung $1:$ Z.O.}

Problemstellung

Frau O. stellt sich seit 2006 regelmäßig aufgrund ihrer psychischen Beschwerden in der Institutsambulanz vor. Aufnahmeanlass war ein seit 15 Jahren bestehendes, umfangreich diagnostiziertes, chronisches Schmerzsyndrom.

\section{Diagnosen}

- Mittelgradige depressive Episode

- Somatisierungsstörung

- Dissoziative Störungen [Konversionsstörungen]

- Posttraumatische Belastungsstörung

62 Vortragsfolie VON LERSNER, Ulrike, 12th European Conference on Traumatic Stress, Wien, 05.06.2011 


\section{Anamnese}

Frau O. klagt über extreme Vergesslichkeit, zeitweise Orientierungslosigkeit und Ängste, die sie zusätzlich zu ihren massiven körperlichen Leiden frustrieren und hinzukommend belasten würden. Frau O. empfinde ihren „Kopf wie einen Stein“. Des Öfteren falle sie in Ohnmacht. Wenn sie nicht in den Garten gehe und sich dort ein wenig beschäftige, versuche sie tagsüber zu schlafen. Ihr Schlaf in der Nacht sei nicht mehr so gestört wie früher, sie schlafe bis zum Morgengebet durch. Ihre Schmerzen seien gleichbleibend stark, keine Behandlung, weder medizinisch noch physiotherapeutisch würde die gewünschte Besserung erbringen. Ihre Arme würden kribbeln, sich wie taub anfühlen. Ihre Beine können sie schwer tragen, sie müsse immer wieder Pausen einlegen und sich z.B. auf Bänke an Bushaltestellen setzen um sich zu erholen. Auch das Atmen fiele ihr schwer. Ihre Aufgaben im Haushalt könne sie kaum noch verrichten, sei mittlerweile selbst auf die Hilfe ihres ebenfalls schwerkranken Mannes angewiesen. Durch ihre Gereiztheit aufgrund dieser Beschwerden komme es vermehrt zu Konflikten innerhalb der Familie. Sie würde in solchen Situationen einfach das Haus verlassen und sich manchmal sogar stundenlang draußen aufhalten - auch nachts. Sie sei kurz davor die Hoffnung, die sie gegenüber den Ärzten hatte aufzugeben.

\section{Psychopathologischer Befund}

Sehr angespannt wirkende, traditionell muslimisch erscheinende, 61-jährige Patientin, die zwar bemüht ist offen und kooperativ Auskunft zu geben - im Verhalten wirkt sie jedoch gequält und überfordert. Das Bewusstsein ist wach, das vermutete Intelligenzniveau normal. Im Gedächtnis zeigen sich Merkfähigkeitsstörungen, sie ist orientiert zu Zeit, Ort, Person und Situation. Die Aufmerksamkeit ist zwar gerichtet, im Affekt aber ängstlich, ablenkbar und sehr schreckhaft. Ferner ist der Affekt deutlich gedrückt. Die Patientin ist nur eingeschränkt schwingungsfähig, weint wiederholt in den Gesprächen. Keine Anzeichen für formale Denkstörungen oder wahnhaftes Erleben, keine Störung des Ich-Erlebens. An Befürchtungen lassen sich allgemeines Misstrauen gegenüber Menschen und Angst vor der Zukunft erfragen. Sie berichtet über 
starke Reizbarkeit. Sie gibt an, oft Gedanken an den Suizid gehabt zu haben eine Umsetzung der Gedanken sei wegen ihres Glaubens nie in Frage gekommen. Kein Konsum von Nikotin oder Alkohol.

\section{Sozialanamnese}

Frau O. sei 1995 nach Deutschland eingereist. Sie lebte bis zu diesem Zeitpunkt im Osten der Türkei in einer dörflichen Region. Sie sei Analphabetin, weil sie nie eine Schule besucht habe. Im Jahre 1964 sei sie im Alter von 15 Jahren verheiratet worden. Sie sei Mutter von 10 Kindern (geboren zwischen 1966 und 1994), wovon die letztgeborenen sechs Kinder in Deutschland leben. Weiter habe sie ein Kind durch eine Frühgeburt verloren und ein Zwillingspaar sei im Kleinkindalter verstorben. Als Muttersprache gibt sie die kurdische Sprache an. Ihre Türkischkenntnisse sind rudimentär, obwohl sie zwar in der Türkei gelebt hat, in ihrem Dorf jedoch ausschließlich kurdisch gesprochen wurde. Ihr Ehemann sei schwer krank, er werde wegen Krebs in der Lunge und im Gehirn seit langer Zeit behandelt.

In ihrem Herkunftsland habe sie, wie bereits erwähnt, keine Schule besucht, wo sie Lesen und Schreiben hätte lernen können. Dieser Umstand frustriere sie sehr. Andererseits scheint sie durch ihre schwere seelische Störung beeinträchtigt im Wiedergeben neuer und alter Informationen. Hauptsächlich klagt sie über Vergesslichkeit, zeitweise Orientierungslosigkeit und Ängste. Eine psychologische Testung zeigte deutliche Hinweise auf Merkfähigkeits- und Reproduktionsstörungen, wobei eine entwicklungsbezogene Intelligenzminderung ausgeschlossen werden kann, da sie bereits vor ihrer Erkrankung in der Lage war, z.B. den Koran in altarabischer Schrift lesen und rezitieren zu lernen.

Für die oben genannten Diagnosen sind Stressintoleranz, Hypervigilanz, Konzentrations- und Merkfähigkeitsstörungen, Ängste (auch soziale Phobien) und von der Patientin beschriebene "Leere im Kopf“ mit damit verbundener Frustrationsintoleranz typisch -bzw. Diagnosekriterien. 


\section{Aktuelle Situation}

Frau O. ist Hausfrau und nicht erwerbstätig. In Deutschland hat Frau $O$. mehrfach an Sprachkursen teilgenommen und versucht die deutsche Sprache zu erlernen. Im Rahmen ihres Einbürgerungsverfahrens war sie aufgefordert einen Kurs mit Erfolg abzuleisten. Diesen Kurs hat sie abgebrochen, weil sie sich zunehmend überfordert fühlte und angab, bis dahin Gelerntes zu verlernen.

\section{Falldarstellung 2: Ö. E.}

\section{Problemstellung}

Frau E. stellt sich seit 2009 in unregelmäßigen Abständen aufgrund ihrer psychischen Beschwerden in der Institutsambulanz vor.

\section{Aktuelle Diagnosen}

- Schwere depressive Episode ohne psychotische Symptome

- Posttraumatische Belastungsstörung

- bei abhängiger Persönlichkeitsstruktur

- Andauernde Persönlichkeitsänderung nach Extrembelastung

- Dissoziative Störungen [Konversionsstörungen], gemischt

\section{Anamnese}

Frau E. klagt über Kopf- Nacken und Schulterschmerzen. Arme und Beine seien manchmal wie taub oder würden kribbeln. Der Kopf sei mit den Gedanken immer hochaktiv, wobei sie ihre Gedanken nicht ordnen könne. Sie könne nicht genug und ruhig schlafen, habe häufig Alpträume. Sie sei immer in Unruhe und beunruhigt es könne ihr jederzeit etwas Furchtbares passieren. Sie empfinde, dass sie ihre Gefühle nicht mehr kontrollieren könne. Weiter berichtet sie von Herzrasen, Schweißausbrüchen, konfusen Gedanken, Selbstwertstörungen und Hemmungen. Sie befände sich 24 Stunden am Tag in einem Alarmzustand, 
während sie gleichfalls oft wie abwesend und fern von der Realität sei. Um nicht passiv zu bleiben, zwinge sie sich, etwas in der Wohnung zu erledigen und leide dabei an großer Angst mit Panikattacken, plötzlich zu versagen, verfolgt zu werden oder zu sterben. Weiterhin berichtet sie über fast tägliche Flashbacks und Intrusionen („Ich lebe immer nur in der Vergangenheit“). Am meisten leide sie unter ihrer Vergesslichkeit und Konzentrationslosigkeit, sie müsse manches wiederholt angehen, weil sie während der Handlung bereits vergesse, was sie beabsichtigte oder würde sich zum Beispiel verlaufen, sofern sie sich überhaupt einmal aus der Wohnung traue. Wegen ihrer starken Erschöpfbarkeit müsse sie immer wieder pausieren.

\section{Psychopathologischer Befund}

Gequält wirkende, religiös verbunden erscheinende, 59-jährige Patientin mit zwar freundlich zugewandtem aber deutlich leidendem Kontaktverhalten. Das Bewusstsein ist wach, das vermutete Intelligenzniveau normal mit im Gespräch eruierbaren Defiziten in der Merkfähigkeit. Zu Zeit, Ort, Person scheint sie orientiert und zur Situation zeitweise nicht orientiert. Motorische Unruhe, z.B. zitternde Hände und häufiges gedankliches Abschweifen ist ebenfalls zu beobachten. Frau E. versucht am Gespräch teilzunehmen, verliert dabei öfter den Anschluss, wirkt dabei wie verträumt (Dissoziation), kehrt dann plötzlich und verschreckt in einen gerichteten Aufmerksamkeitszustand zurück. Ihre Affektivität ist geprägt durch eine durchweg gedrückte nahezu weinerlich-jammernde Haltung. Es bestehen keine Anzeichen für formale Denkstörungen oder wahnhaftes Erleben, keine Störung des Ich-Erlebens. Sie gibt an sehr misstrauisch gegenüber Menschen im Allgemeinen (auch ihrer Familie gegenüber) zu sein, über ihre Zukunft nur negative Gedanken zu haben und viel darüber zu grübeln. Ihre innerliche Wut und Angespanntheit würden zunehmend zu einem innerlichen und äußeren Rückzug führen damit Konflikte mit anderen (z.B. Familienmitgliedern) nicht exazerbieren. In vielen Situationen ihres Rückzuges habe sie immer wieder Suizidgedanken und -pläne, die sie jedoch aus Mutlosigkeit nicht hätte umsetzen können. Nikotin oder Alkohol würde sie nicht konsumieren. 


\section{Sozialanamnese}

als Frau E. noch ein kleines Kind war habe ihr Vater ihre Mutter und ihre vier Geschwister wegen einer anderen Frau über Nacht verlassen und mittellos gelassen. Sie seien damit in ihrem Dorf auf sich alleine gestellt gewesen - ohne Hilfe der Verwandtschaft. Als Frau E. 16 Jahre alt war starb die Mutter, sodass sie für die Versorgung ihrer weiteren vier Geschwister in der Vater- und Mutterrolle verantwortlich war. Eine Schule habe sie in der Türkei wegen ihrer Armut nur bis einschließlich zur vierten Klasse besucht, obwohl sie gerne weitergelernt hätte. Mit 18 Jahren wurde sie an ihren jetzigen Ehemann vermittelt verheiratet. Sie habe ihn erst am Tag ihrer Hochzeit kennen gelernt, mit ihm 5 Monate zusammengelebt, bis er 1972 als Gastarbeiter nach Deutschland ausgereist sei. Er habe sie bei seinen Eltern gelassen, die sie ca. 20 Jahre lang jeweils bis zu deren Tod gepflegt habe. Sie habe dort „Sklavenarbeit“ leisten müssen; sich um die Landwirtschaft kümmern, kranke Alte pflegen („Windeln, Füttern,...“). Ihre vier Kinder habe sie alleine ohne weitere Hilfe zur Welt gebracht. Ihre erste Tochter sei mit 23 Jahren verstorben und habe zwei kleine Kinder hinterlassen. Ihre zweite Tochter, habe sie nicht gut versorgen können, sodass diese mit 4 Monaten verstarb. Später habe sie zwei Söhne geboren. Der jüngere der beiden Söhne lebe noch mit ihr zusammen in der 2-Zimmer Wohnung der Eltern. Sie habe, bis sie 1996 nach Deutschland nachgeholt wurde, alleine mit ihren Kindern gelebt und ihren Mann manchmal bis zu vier Jahre nicht gesehen. Im Jahr 2002 habe sie versucht einen Deutschkurs zu belegen, den sie dann nach einem Monat wegen Überforderung mit mannigfaltigen körperlichen Symptomen aufgab.

\section{Aktuelle Situation}

Frau E. leidet unter Symptomen einer psychotraumatischen Störung, die verschiedene Auslöser hat. Eine frühe Parentifizierung zum einen, Verlust zweier Kinder, wofür sie sich verantwortlich (schuldig) macht zum anderen und ein Ehemann, der gegen sie und ihre Kinder über Jahre heftige Gewalt angewendet hat, die bis hin zu körperlichen Verletzungen ging.

Frau E. in ihrer Rolle als Puffer für ihre Söhne, die sie schützen möchte, als 
hilflose Großmutter ihrer Enkelinnen, die sie unterstützen möchte und als unterdrückte Frau extrem überfordert und ihrer Situation ausgeliefert. Eine Trennung habe sie oft in Erwägung gezogen, sich aber aufgrund der sozialen Abhängigkeit vom Mann, ihrer traditionellen Prägung und ungewissen Zukunftsperspektiven dagegen entschieden. Sie verarbeitet ihre Situation als Schicksalsschlag unter dem sie leide.

Aufgrund der wahrgenommenen Stressoren und ihrer daraus resultierenden Störung ist sie nicht in der Lage trotz mehrfacher und ressourcenraubender Bemühungen die deutsche Sprache besser zu erlernen. Unter gegeben schweren Voraussetzungen als (Quasi-) Analphabetin ohne längere Schulerfahrung hat sie mehrfach an Sprachkursen teilgenommen. Sie hat dabei unter für sie belastenden Situationen den Mut gefunden, die deutsche Sprache zu erlernen und zu sprechen. Mehr als in den Kursen habe sie sprachlich am meisten von Nachbarn oder beim Einkauf in Geschäften profitieren können.

Nach einer Aufforderung zum verpflichtenden Besuch eines Kurses für die Aufrechterhaltung des Aufenthaltstatus klagt Frau E. vermehrt über Vergesslichkeit, zeitweise Orientierungslosigkeit und Ängste, die sie zusätzlich zum Umstand des Nichtanerkennens ihrer bisherigen Leistungen und ihrer massiven körperlichen Leiden frustrieren bzw. zusätzlich zu ihrer aktuellen sozialen/ familiären Situation belasten.

\section{Falldarstellung 3: Z.Y.}

\section{Problemstellung}

Herr Y. stellt sich seit 2006 regelmäßig aufgrund seiner psychischen Beschwerden in der Institutsambulanz vor. Aufnahmeanlass war eine Anpassungsstörung und eine schwere depressive Phase ohne psychotische Symptome. 


\section{Anamnese}

Herr Y. berichtet über Ängste, Schlafstörungen mit Alpträumen, manchmal so schwer, dass er schreiend und schweißüberströmt aufwache. Er sei sehr unkonzentriert, sehr vergesslich, grübele viel, könne viele Menschen an einem Ort nicht aushalten, streite öfter schon mit der Familie und habe sich aus diesem Grund zunehmend aus seinem Freundeskreis zurückgezogen. In dem Café, in dem er sich früher mit seinen Freunden und Bekannten bisher regelmäßig getroffen habe, könne er sich wegen zunehmender Gereiztheit und verbaler Auseinandersetzungen, die durch inn bedingt entstehen würden und bis zu Handgreiflichkeiten führen können, nicht mehr sehen lassen. Innerlich sei er sehr angespannt, verspüre Unruhe, Nervosität. $\mathrm{Er}$ berichtet weiterhin über Kopfschmerzen, Brennen in den Augen, Gereiztheit und Schuldgefühle. Er könne Menschen nicht mehr trauen, fühle sich häufig wie „leer" und verlassen. Oft sei er innerlich aggressiv und energiegeladen, könne dies aber nicht herauslassen, fühle sich trotzdem dadurch erschöpft. Zunehmend ist eine Verschiebung auf eine Körpersymptomatik festzustellen, die sich in Äußerungen über Beschwerden von Schmerzen im Bauch, Beinen, Rücken und Kopf darstellt.

\section{Diagnosen:}

- Mittelgradige depressive Episode

- Posttraumatische Belastungsstörung

- Dissoziative Störungen [Konversionsstörungen]

- Somatisierungsstörung

- Andauernde Persönlichkeitsänderung nach Extrembelastung

\section{Psychopathologischer Befund}

Sehr angespannt wirkender 52-jähriger, freundlicher und redseliger Patient. Das Bewusstsein ist wach, das vermutete Intelligenzniveau normal. Das Gedächtnis zeigt sich intakt, er ist zu Zeit, Ort, Person und Situation orientiert. In der Aufmerksamkeit ist er zwar konzentriert, im Antrieb wirkt er jedoch eher gedrückt und dabei motorisch unruhig. Der Affekt ist etwas gedrückt und 
schwingungsfähig. Es bestehen keine formalen Denkstörungen. An Befürchtungen lässt sich allgemeines Misstrauen gegenüber Menschen erfragen. Hinweise für Sinnestäuschungen oder Wahninhalte lassen sich nicht eruieren. Er berichtet über immer wiederkehrende extreme Flashbacks und intrusive Zustände bis hin zu dissoziativem und depersonalisiertem Erleben, berichtet über Ein- und Durchschlafstörungen und extreme Reizbarkeit. Es besteht latente Suizidalität. Er gibt an, oft Gedanken an den Suizid zu haben. Konsum von Nikotin und Alkohol in der Vergangenheit „um sich zu beruhigen“ (Nikotinabstinenz seit zwei Jahren), keine anderen Drogen.

\section{Sozialanamnese}

Herr Y. ist vorletztes Kind von neun Geschwistern. Die beiden ältesten Geschwister seien 1965 und 1986 verstorben. Der Vater sei 1965 durch einen Blitzschlag und die Mutter wegen Herzinsuffizienz 1986 verstorben. Er hat die Mittelschule in der Türkei abbrechen müssen (ca. 7. Schuljahr), scheint sich jedoch durch Selbstbildung weiterentwickelt $z u$ haben und sehr belesen (u.a. Dostojewski, Marx, Kant) zu sein. Seine Heimat sei in der Türkei die Stadt Tunceli, er habe dort in verschiedenen Städten gelebt, zuletzt in Izmir. Als Beruf gibt er Maler an, er sei in der Türkei aber auch anderen Tätigkeiten nachgegangen.

Geheiratet habe er 1982. Er habe zwei erwachsene Söhne und eine Tochter. Im Jahre 1993 sei er mit seiner Familie als Flüchtling nach Deutschland eingereist. Er spricht Kurmanji, Zaza und Türkisch.

\section{Aktuelle Situation}

Der Patient klagt über Leere im Kopf nach erlebter Folter. Auch Flashbacks und intrusive Zustände seien vorhanden. Weiterhin berichtete der Patient von Kraftlosigkeit, Grübeln, geistiger Abwesenheit, Orientierungslosigkeit, Misstrauen gegenüber anderen Menschen bis hin zu sozialen Phobien. Außerdem leide er an extremer Vergesslichkeit, was zu Problemen im Deutschkurs führe, den er besuche, um die deutsche Staatsbürgerschaft zu erlangen. Seine Kursleiterin habe inm eine therapeutische Behandlung empfohlen, weil er sich durchwegs unter Druck gesetzt fühle, antriebslos jedoch gleichzeitig gereizt sei. Durch den 
Kontakt zu Deutschen habe er seinen Angaben nach ausreichend gut Deutsch gelernt, könne sich sehr gut mit innen verständigen und unterhalten, während seine Leistungen im Kurs auch für sein Empfinden nicht akzeptabel seien. Die Prüfung werde er unter diesen Umständen nicht bestehen können.

Zur Stressfolgestörung kommen bei diesen Fallbeispielen die Stressoren für die Akkulturation dazu. Um mit ihrer Lebenssituation zurechtzukommen haben sie für sich wirksame gute Copingstrategien und gute Bewältigungsmuster in der Vergangenheit gehabt bis die Anforderung nach dem Lernen hinzukam. Diese Anforderung verstärkt in der neuen Situation die Gesamtpathologie. Dass die Menschen in den Fallbeispielen 'Nicht-Lerner' sind ergibt sich aus inrer biographischen Darstellung. Der Kontext des Lernens war bei allen dreien ein anderer: ihrer Lebenswelt angepasst und ohne externen 'Druck'. Dementsprechend konnten sie auch Lernleistungen erbringen, wie z.B. mit Koran lesen lernen, Sprachenlernen und sich inhaltlich mit Literatur beschäftigen.

\section{3 Übersicht über Abbruchstatistik von Integrationskursen}

Im Folgenden soll anhand der Abbruchstatistik bei Integrationskursen diskutiert werden, wie bzw. ob sich die subjektive externe Anforderung dadurch als möglicher Stressor auswirken kann.

$\mathrm{Zu}$ den Integrationskursen führt das Bundesamt für Migration und Flüchtlinge in seinem Bericht zur Integrationskursgeschäftsstatistik für das Jahr $2010 \mathrm{an}^{63}$ :

„Seit Einführung der Integrationskurse am 01.01.2005 wurden bis zum 31.12.2010 insgesamt

rund 918.000 Teilnahmeberechtigungen und -verpflichtungen erteilt. 690.000 Teilnehmer haben einen Integrationskurs besucht oder besuchen inn gegenwärtig.

[...]

Der Integrationskurs richtet sich vor allem an Neuzuwanderer, wird in der Praxis aber als ein wichtiges Instrument der "nachholenden Integration“ genutzt und dient denjenigen Menschen als Hilfestellung, die bereits länger in Deutschland leben und noch nicht über ausreichende Sprachkenntnisse verfügen. Es ist jedoch absehbar, dass der prozentuale Anteil dieser Gruppe an den Teilnehmern - derzeit

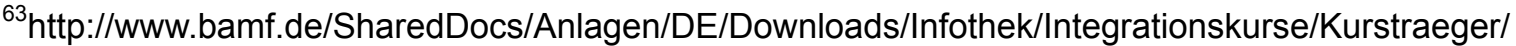
Statistiken/2010-integrationskursgeschaeftsstatistik-de.pdf?_blob=publicationFile , letzter Zugriff: 16.06.2011
} 
bei 63 Prozent liegend - in den nächsten Jahren strukturell abnehmen wird, da nachwachsende Generationen das deutsche Bildungssystem durchlaufen und auf diese Weise von klein auf sprachlich gefördert werden." (Bericht zur Integrationskursgeschäftsstatistik für das Jahr 2010, S. 2)

In der Darstellung der Veränderungen der Abbrüche des Integrationskurses (Tabelle 5) lässt sich z.B. ein Anstieg vom Jahr 2008 bis 2009 von nicht-erfolgreich Abschließenden bei gleichzeitig deutlich steigender Zahl von Kurswiederholern ersehen. Auch die Zahl der Absolventen reduziert sich - dies lässt sich als Anstieg von ,Abbrechern' des Kurses interpretieren.

Tabelle 5 Abbruchstatistik nach BAMF ${ }^{64}$

\begin{tabular}{|c|c|c|c|c|c|c|c|}
\hline Abbruchstatistik & 2008 & in \% & 2009 & in $\%$ & $\begin{array}{l}\text { Vergleich } \\
(08 / 09)\end{array}$ & $\begin{array}{l}01.01 .- \\
30.9 .2010 \\
\end{array}$ & in $\%$ \\
\hline $\begin{array}{l}\text { Teilnahmeberechtigt } \\
\text { für Kurswiederholer }\end{array}$ & $\begin{array}{r}155.504 \\
30.277 \\
\end{array}$ & & $\begin{array}{r}145.934 \\
33.367 \\
\end{array}$ & & $\begin{array}{r}-6,2 \% \\
+10,2 \% \\
\end{array}$ & $\begin{array}{l}87.016 \\
18.977 \\
\end{array}$ & \\
\hline Summe & 185.781 & & 179.301 & & $-3,5 \%$ & 105.993 & \\
\hline $\begin{array}{l}\text { Kursteilnehmer } \\
\text { Teilnehmende Wiederholer }\end{array}$ & $\begin{array}{r}121.275 \\
20.396\end{array}$ & $\begin{array}{r}78 \% \\
67,4 \%\end{array}$ & $\begin{array}{r}116.052 \\
27.174\end{array}$ & $\begin{array}{l}79,5 \% \\
81,4 \%\end{array}$ & $\begin{array}{r}-4,3 \% \\
+33,2 \%\end{array}$ & $\begin{array}{l}69.556 \\
18.852\end{array}$ & $\begin{array}{l}79,9 \% \\
99,3 \%\end{array}$ \\
\hline Summe & 141.671 & $76,3 \%$ & 143.226 & $79,9 \%$ & $+1,1 \%$ & 88.408 & $83,4 \%$ \\
\hline davon Türken & 21.475 & & 19.245 & & $-10,4 \%$ & 9.623 & \\
\hline Kursabsolventen insgesamt & 73.557 & $51,9 \%$ & 70.968 & $49,5 \%$ & $-3,5 \%$ & 61.377 & $69,4 \%$ \\
\hline davon Türken (größte Gruppe) & 13.241 & & 10.893 & & $\begin{array}{r}15,3 \% \\
\text { (aller } \\
\text { Absolventen) }\end{array}$ & 9.361 & $15,3 \%$ \\
\hline Nicht erfolgreich abgeschlossen & 68.114 & $48,1 \%$ & 72.258 & $50,5 \%$ & $+5,7 \%$ & 27.031 & $30,6 \%$ \\
\hline $\begin{array}{l}\text { Berechtigt, aber Nichtteilnehmer } \\
\text { Berechtigt, aber Nichtwiederholer }\end{array}$ & $\begin{array}{r}34.229 \\
9.881\end{array}$ & $\begin{array}{r}22 \% \\
32,6 \% \\
\end{array}$ & $\begin{array}{r}29.882 \\
6.193\end{array}$ & $\begin{array}{l}20,5 \% \\
18,6 \%\end{array}$ & $\begin{array}{l}-12,7 \% \\
-37,3 \%\end{array}$ & $\begin{array}{r}17.460 \\
125\end{array}$ & $\begin{array}{r}20,1 \% \\
0,7 \%\end{array}$ \\
\hline Summe & 44.110 & $23,7 \%$ & 36.075 & $20,1 \%$ & & 17.585 & $16,6 \%$ \\
\hline Begonnene Integrationskurse & 9.219 & & 9.146 & & $-0,8 \%$ & 6.102 & \\
\hline Beendete Integrationskurse & 5.743 & & 6.725 & & $+17,1 \%$ & 4.705 & \\
\hline
\end{tabular}

Laut dem Abschlussbericht und Gutachten über Verbesserungspotenziale bei der Umsetzung der Integrationskurse des Bundesministeriums des Innern (Evaluation der Integrationskurse nach dem Zuwanderungsgesetz, 2006) ${ }^{65}$ schließen nur $40 \%$ der Teilnehmenden erfolgreich ab, da mindestens ein Kursteil nicht absolviert wird. Unter anderem werden dafür psychische oder andere

\footnotetext{
${ }^{64}$ http://www.bamf.de/SharedDocs/Anlagen/DE/Downloads/Infothek/Integrationskurse/Kurstraeger/ Statistiken/2010-integrationskursgeschaeftsstatistik-de.pdf?_blob=publicationFile , letzter Zugriff: 16.06.2011

${ }^{65} \mathrm{http}: / /$ www.integration-indeutschland.de/cln_117/nn_1640754/SharedDocs/Anlagen/DE/Integration/Downloads/Integratio nskurse/Kurstraeger/Sonstiges/abschlussbericht-

evaluation,templateld=raw,property=publicationFile.pdf/abschlussbericht-evaluation.pdf, letzter Zugriff: 16.06 .2011
} 
gesundheitliche Probleme, Aufnahme einer Beschäftigung oder Schwangerschaft als Grund für den Abbruch angegeben.

Als Determinanten des Kurserfolgs werden Zusammenhangsmuster bzgl. der Teilnahme am Abschlusstest betrachtet:

- Teilnehmende am Abschlusstest haben signifikant häufiger ein höheres Bildungsniveau

- Teilnehmende an Abschlusstest haben signifikant häufiger ein Vorliegen von mindestens einfachen Sprachkenntnissen

- Es besteht eine positive Korrelation eines hohen Punktestands im Einstufungstest und der Teilnahme am Abschlusstest

- Je höher die Lernprogression ist, desto höher ist die Wahrscheinlichkeit am Abschlusstest teilzunehmen

- bei Kursbeginn nicht Alphabetisierte nehmen signifikant seltener als alphabetisierte Kursteilnehmer/innen am Abschlusstest teil.

Das Bildungsniveau kann demnach als zentraler Einflussfaktor für einen erfolgreichen Kursabschluss angesehen werden.

Interessant sind ebenso einige Aspekte, die keinen nennenswerten Einfluss auf die Teilnahme am Abschlusstest haben:

- Geschlecht

- Aufenthaltsdauer und -status

- Nationalität

- Alter

- Muttersprache

Nach Einschätzung der meisten Teilnehmer/innen (ca. 40\%) war der Zugewinn an Sprachkenntnissen durch die Integrationskurse dabei mittelgroß, nahezu 30\% der Befragten hatten nach eigenen Angaben viel hinzugelernt. Daraus resultierend kann gefolgert werden, dass einige weitere Befragte keine Angaben gemacht haben oder keinen Zugewinn an Sprachkenntnissen erzielt haben ${ }^{66}$.

Als Stressoren sollten an dieser Stelle ebenfalls, neben den inhaltlichen Anforderungen des Integrationskurses auch die Sanktionen für die Nichtteilnahme oder des Abbruches erwähnt werden:

Bei Nichtteilnahme oder Abbruch des Integrationskurses ist bereits nach der geltenden
Rechtslage ein abgestuftes System von Sanktionen eingeführt. Ist der Teilnehmer Bezieher
von Arbeitslosengeld II, kann ihm dieses um $30 \%$, bei wiederholten Verletzungen der
Teilnahmepflicht auch darüber hinaus gekürzt werden. Weitere Maßnahmen sind u.a. die
Verhängung eines Bußgeldes und als Ultima Ratio die Nichtverlängerung der
Aufenthaltserlaubnis. Es obliegt der jeweiligen Ausländerbehörde bzw. dem Träger der
Grundsicherung, festzustellen, ob eine Verletzung der Pflicht zur Teilnahme an einem
Integrationskurs vorliegt bzw. festzulegen, ob und welche Sanktionen verhängt werden."
(Quelle BAMF)

${ }^{66}$ Eine Nachfrage hierzu im September 2010 beim BAMF hat bisher noch keine Antwort erbracht.

67 http://www.integration-in- 
Die auffällig hohe Zahl an Abbrechern innerhalb der Integrationskurse lässt sich, in Verbindung mit den oben angeführten Falldarstellungen (Abschnitt III/2.2), leicht nachvollziehen und fordert gewissermaßen aus sich heraus bereits eine Reform an der Form der Durchführung der Integrationskurse. Trotz der ausgesprochenen Sanktionen, die zum Teil subjektiv als existentiell erlebt werden (z.B. Gefährdung des Aufenthaltsstatus), kommt es dennoch bei nicht unerheblich vielen Teilnehmern zu Abbrüchen.

\section{Zwischenfazit}

Aus den Ergebnissen der Erhebung mit dem Fragebogen zur Lebenszufriedenheit, der Fallbeispiele und der Aufbereitung der Abbruchstatistik der Integrationskurse, lässt sich folgern, dass Menschen mit Zuwanderungsgeschichte -insbesondere im Alter- Stressoren durch äußere Faktoren und Situationen ausgeliefert sind, die durch ihre Fremdheit bzw. ihrem Status als 'Ausländer' begründet sind. Es resultiert daraus eine als allgemein zu betrachtende niedrige Lebenszufriedenheit bei gleichzeitiger Erwartungshaltung an sie, eine 'Integrationsleistung' in Form von Lernen zu erbringen. Dieser Erwartungshaltung können sie nicht folgen, da sie auf der einen Seite zumeist nicht bildungsorientiert geprägt sind, auf der anderen Seite zur Aufrechterhaltung ihrer Identität und Lebenszufriedenheit wenig Anreize gefunden haben sich mit dem (systematischen) Lernen (z.B. der deutschen Sprache) auseinanderzusetzen. Es gilt demzufolge Konzepte für Menschen mit Migrationsgeschichte zu entwickeln, die eine Atmosphäre des Lernens schaffen und nicht entsprechend den Ausführungen neurobiologischer Ansätze, keine als Stressor dienende inadäquate Anforderungen stellen.

deutschland.de/nn_442622/SharedDocs/Pressemitteilungen/DE/DasBAMF/2010/100928-0017pressemitteilung-ik-zahlen.html, letzter Zugriff: 16.06.2011 


\title{
C. Fazit und Ausblick
}

\section{Zusammenfassung und abschließende Überlegungen}

Die Wanderung durch diese Arbeit endet nun in einer Zusammenfassung und schließt mit weiterführenden Überlegungen. Da einleitend mit einem Zitat Johann Wolfgang von Goethes begonnen wurde, bietet es sich an, den Bogen zu dem ehemaligen Binnenmigranten (von Frankfurt nach Weimar) auch zu schließen. Johann Wolfgang von Goethe war ein Tausendsassa. Ein sogenanntes Genie. Forscher mit Herzblut in verschiedenen Disziplinen: von der Biologie (seine Liebe zum Gingko Biloba dürfte unverkannt sein), über die Medizin (er entdeckte den Zwischenkieferknochen), die Farbenlehre, Optik, Kunstsammlung und Zeichenkunst bis hin zur weltberühmten Schriftstellerei erstreckte sich sein Interessengebiet. Vor allem war er aber aufgrund seiner vielen Interessen ganzheitlich orientiert und interessiert. Hätte Goethe das Plädoyer dieser Arbeit in nuce zusammenfassen wollen, hätte er sich im Jahr 2007 von AUERNHEIMER inspirieren lassen können, der für eben diese Ganzheitlichkeit - wie es hiermit diese Arbeit auch tut - plädierte:

\begin{abstract}
Inzwischen hat sich - genauso wie in Großbritannien - weitgehend die Einsicht durchgesetzt, dass es eines ganzheitlichen Ansatzes bedarf, d.h. dass eine Atmosphäre von Vertrauen und Akzeptanz geschaffen werden muss, damit Lernwiderstände überwunden werden und die Lernenden offen für Argumente sind. Exemplarisch dafür kann Bärbel Kampmanns (1999) vorgestelltes Konzept stehen, in dem sie betont, wie entscheidend es ist, dass die Lernenden vor allem in der ersten Phase ihre Erwartungen, Ängste und Sorgen artikulieren können, und dass es innen möglich sein müsse, mit Vorurteilen und Stereotypen herauszurücken. Erst schrittweise sei ein mehr kognitiver Zugang möglich. (AUERNHEIMER 2007: 156)
\end{abstract}

Ganzheitlichkeit ist keineswegs esoterisch ${ }^{68} \mathrm{zu}$ verstehen, sondern gemeint im Grundverständnis der Wortbedeutung. Der Mensch als Ganzes muss bei der Entwicklung jedweder den Menschen betreffenden Konzepte, aber insbesondere bei der Entwicklung didaktischer Ansätze berücksichtigt werden. Die in dieser Arbeit verknüpften Aspeke Alter, Lernen und Neurobiologie - insbesondere wenn es sich um (analphabetische) Migranten handelt - können, bei Berücksichtigung der Verbindungen, gewinnbringend (im sozialen, psychischen, politischen, ökonomischen Sinn etc.) eingesetzt werden. Diese Arbeit soll einen Beitrag dazu

68 Esoterik kann man im weitesten Sinne natürlich im Fall der türkischstämmigen Migranten anführen, wenn Religion thematisiert wird, da eine Religionsgemeinschaft zu einer guten, positiven Atmosphäre beitragen kann, oder gar motivieren eine weitere Sprache wie altarabisch zu lernen, um den Koran zu verstehen. 
leisten, den von AUERNHEIMER beschriebenen Prozess der Einsicht hinsichtlich eines ganzheitlichen Ansatzes zu vertiefen, um durch die Verschmelzung der Gegenstandsbereiche Migration, Alter, Lernen und Neurobiologie insbesondere der Didaktik eine weitere stützende Säule bieten zu können, die bereits in vielen Teildisziplinen und Ansätzen ganzheitlich arbeitet.

Zwar ist in der vorliegenden Arbeit das Thema Lernen vordergründig im Fokus, jedoch ist Lernen, wie bereits ausgeführt, ein lebenslanger Prozess. Also beschäftigte sich diese Arbeit, ebenso wie alle Disziplinen die sich mit dem Thema Lernen, Migration, Alter oder Stress beschäftigen, direkt oder indirekt mit dem Thema Leben.

Maßgeblich steht die Verbesserung der Lebensqualität und Lebenswelt (siehe z.B. Fragebogen-Studie) durch Berücksichtigung aktueller Ergebnisse der Hirnforschung - oder mit MURRAY (1977) auch schon deutlich vor dem Aufstreben der Hirnforschung - im Zentrum dieser Arbeit.

Die Dissertation zielt darauf ab, bei jedweder Art von Konzept zur Lebensqualitäterhöhung, beispielsweise durch Stressreduktion, auch diese Fakten miteinzubeziehen und einer Zielgruppe wie den alten Migranten kulturangepasste Lernangebote zu offerieren. So schreibt MURRAY zum Thema Lernen bereits 1977:

\footnotetext{
Experiental Learning, whatever else it is, attempts to encompass both brain-functions, and so is left crippled, as it were, one leg firmly rooted in the analytical tradition, one leg hanging in the void. It is into this void I would like to take a tentative plunge. To start with, it would be helpful to have some specific examples of what we mean by experiental learning. Here are three in which I have been recently involved. (Murray 1977: 37f)
}

MURRAY stellt somit bereits früh dar, dass das Experiental Learning den Versuch unternimmt, beide Hirnfunktionen gleichermaßen zu umfassen. Weiter führt er aus, dass somit das Experiental Learning mit einem Bein fest auf dem Boden der analytischen Tradition steht, während es mit dem zweiten Standbein im Leeren hängt. MURRAY (vgl. 1977: 37f) schlägt zum Untersuchen dieser Leere eine Methode vor, die er als 'processing' bezeichnet und die in ihrem Grundverständnis das von AUERNHEIMER und auch HÜTHER postulierte Bedürfnis nach einer guten, das Lernen ermöglichende räumlich-sozial-interaktionistisch-emotionalen, kurz: ganzheitlichen Atmosphäre beschreibt. Diese von MURRAY vorgeschlagenen Gesprächsrunden zeichnen sich dadurch aus, dass freiwillig an ihnen teilgenommen wird, die Interaktion innerhalb der Gruppe nicht nur auf verbale Interaktion beschränkt ist, sich die gesamte Gruppe wahrheitsgemäß so weit möglich über ihre Gefühle äußert und jedes aufkommende Thema ausführlich 
behandelt wird. Die Themen, die Situationen außerhalb des Treffens betreffen, werden im Gespräch als Teil eines größeren Prozesses verortet, indem die Schritte die zu der entsprechenden Situation oder dem entsprechenden Ereignis (welches das Thema darstellt) geführt haben und die Schritte die danach erfolgten. Dieses 'processing' dient dazu das Experiental Learning im Modell nach zu stellen. Bemerkenswert ist dabei, dass sowohl die freiwillige Teilnahme, als auch die uneingeschränkte Interaktion wichtig sind um sicherzustellen, dass das Processing nicht als Stress empfunden wird:

One way to approach this void which I have found useful is "processing." This need not be a cover-up for an informal rap session; it can be a valuable tool to replace traditional learning structures (classes), evaluation (grades) and competition (comparison), all unsuited to experiental learning. In my experience a good process session includes some of the following characteristics: people participate voluntarily; Interaction is not limited to verbal; At the outset, one person (usually the leader) takes responsibility for focusing group energy around a significant, non-superficial issue;

Participants agree to speak the truth, as far as possible, about their feelings. It is crucial that the leader not be exempt from this agreement, but engaged fully as a participant, modeling the frank and sometimes risky expression of feelings. At the same time the leader should take responsibility to be sensitive to the vulnerability created by such exposure, and to make sure the consequences of that vulnerability get dealt with; participants agree to deal with whatever comes up in a thorough way. That is, an atmosphere of security is established to avoid someone's being left hanging, with some feelings expressed but not fully dealt with; The issue is an "outside" situation or event, and the processing session connects steps people are taking before and after; it clarifies events in such a way that links them together and gives them direction in a larger process which is the actualization of individual or shared values, goals, objectives. (The articulation of these values and goals is an important on-going group task);

"Inside" issues which arise with the session are also dealt with. Attention is periodically directed to the spontaneous here-and-now feelings and responses of participants.

So a good process session successfully models good experiental learning, in that it deals with spontaneous feelings in an interpersonal setting. It is the most highly structured time in this it resembles the traditional class - but it uses structure not to confine learning but model it in a way that can be carried on in an attenuated form in the normal daily life of the participants. At the same time it helps evaluate learning in a dual sense. First, people get in touch with what is really going on at a non-superficial level - what they are in fact valuing internally by their behavior and responses, in daily life and in the group. And secondly, there is a chance to share what they value in theory and externally, by articulating goals and objectives. If there is a discrepancy between actual internal valuation (preoccupation) and ideal values (goals), then there is a chance to share ideas of steps to be taken to bring the actual closer to the ideal. (ebd.)

\section{Somit beschreibt MURRAY was diese Arbeit in nuce aussagen will:}

Lernen ist mehr als der unbedingte Wunsch etwas aufzunehmen, abhängig von der Atmosphäre und vor allem mehr als reine neurobiologische Verarbeitung von Informationen - wenngleich die neurobiologischen Prozesse in der Art erhöhte Beachtung finden müssen, dass eine neurobiologische Blockade beim Lernen umgangen werden kann, z.B. durch die von HÜTHER postulierte 'richtige Atmosphäre'. Zusammenfassend bedeutet das: gutes, nachhaltiges und stressfreies (und damit neurobiologisch überhaupt mögliches) Lernen erfordert die 
für jeden Lernenden 'richtige' kultur-, alters-, sozial-, und u.a. bildungsangepasste Atmosphäre. Bemerkenswert ist, dass MURRAY explizit anführt, dass gerade die nonverbalen Elemente seiner Methodik basal sind. Nonverbal im weitesten Sinne ist auch der 'Infokoffer', der im zweiten Abschnitt des Fazits nochmals im Zentrum des Interesses stehen soll.

Den Infokoffer kultur-unangepasst zu benutzen, also beispielsweise durch jemanden vermitteln zu lassen der von der Zielgruppe kultur- oder sozialbedingt (durch beispielsweise eine andere Sprache, soziale Stellung, kulturellen Hintergrund etc.) nicht akzepiert sein könnte, erscheint sinnfrei, wenn man sich vor Augen führt:

Kompetenter Umgang mit kulturellen Differenzen verlangt zum einen die Bearbeitung von Irritationen und von den Problemen, einander zu verstehen [...], zum anderen aber auch den Dialog über umstrittene Geltungsansprüche von religiösen Werten, Geschlechterrollen etc. - oder auch einfach von Kommunikationsregeln. (AUERNHEIMER 2007: 137)

Versteht man das Leben in der Migration als Leben in einer fremden kulturellen Gesellschaft mit obigen kulturellen Differenzen, können dabei verschiedene Stressoren zu Tage treten. Zum einen können z.B. nach BowLBY (1975) Bindungsstörungen auftreten, die zu Stress führen, da

die Entwicklung von emotionalen Bindungen als eine der menschlichen Natur inhärente Eigenschaft. Der Mensch ist neben einer Reihe anderer Verhaltenssysteme damit ausgestattet, dass sich im Laufe der Phylogenese/Enwicklung zur Sicherung des Überlebens ausgebildet hat. Die Bedeutung der frühkindlichen Beziehung zu den primären Bezugspersonen und die Ausbildung der Persönlichkeitsstruktur wurde in den vergangenen Jahren Gegenstand zahlreicher empirischer Studien. (BoWLBY, 1975)

Dies ist insofern problematisch, da Beziehungserfahrungen bereits früh als stabile, generalisierte Erwartungsstrukturen in der Beziehung zu einer Person ausgebildet werden (z.B. im Kleindkindalter zunächst in nonverbaler Gedächtnisform cerebral gespeichert) und sie prägen das Interaktionsverhalten (vgl. WALLER/SCHEIT 2002: 159). Hirnbiologische Veränderungen können hiervon, aber auch von Akkulturationsstress die Folge sein, die sich wiederum auf die Lernfähigkeit auswirken können. Neben den bisherigen Problemen elementar sind jedoch mögliche Anpassungsstörungen die eine Lebensqualitätsveränderung zur Folge haben können, die im schlimmsten Fall psychosomatische und psychologische Störungen mit sich bringen.

Anpassungsstörungen, Lebensqualtitätsveränderungen oder Bindungsstörungen sind Faktoren, die die Lebenswelt der Migranten verändern und ein verändertes Verhalten in der Migration als folge haben. Diese wird daraus 
resultierend als Misserfolg und persönlichkeitsstörender Umstand im Erleben und Verhalten wahrgenommen. Auf die oben genannten Punkte wird sich dies wiederum dysfunktional auswirken und schlussendlich entsteht durch die mögliche Wahrnehmung des vermeintlichen Misserfolgs der Migration erneut Stress.

Wenn man nun für einen didaktischen Zugang all das berücksichtigen möchte, bräuchte man für die Umsetzung bei alten Migranten, oder beispielsweise bei seit kurzer Zeit in Deutschland lebenden Flüchtlingen ohne deutsche Sprachkenntnisse Lernen und Bildung zu ermöglichen, Faktoren, die den speziellen Bedürfnissen dieser Zielgruppen holistisch nachkommen. Im Bereich didaktischer Konzepte kann dem Sorge getragen werden, indem man sich die oben genannten Faktoren bewusst macht und entsprechend umsetzt, zum Beispiel anhand von:

- altersgerechten Konzepten

- genderspezifischen Konzepten, beispielsweise Sprachunterricht für türkischstämmige Frauen mit religions- und kultursensiblen Kleingruppen (z.B. reine Frauengruppen)

- sprachreduzierten ${ }^{69}$ Konzepten, wie Coemenius / Didaktika Magna / Orbus pictus / Matetik

- Alphabetisierung berücksichtigenden Konzepten

- auf Bindung während des Lernens fokussiernden Konzepten: z.B. nach HÜTHER eine Atmosphäre (Klima) für das Lernen zu schaffen und die verschiedenen Zugänge berücksichtigen

- neurobiologische Aspekte berücksichtigenden Konzepten, die die Aspekte Herdenverhalten nach HÜTHER, Bindungstheorie, intrinsische Motivation zum Lernen oder auch antizipatorisches Lernen beinhalten.

Begründet finden sich diese Wünsche an eine ganzheitliche Didaktik in den vorangegangenen Kapiteln Migration, Alter, Lernen, Neurobiologie und in den Studien, Statistiken und Fallbeispielen. Im Text wurde gleichfalls eine didaktischpädagogische Möglichkeit vorgestellt, die die erforderlichen Aspekte

69 Sprachreduzierte Konzepte dienen dem leichteren Lernen und damit eröffnen sie langfristig die Möglichkeit über intrinsische Motivation, da der Stres des Fremdsprachenlernens reduziert ist, die Sprache im Zielland aus eigenem Antrieb und mit Freude für sich selbst zu lernen und damit gleichzeitig positiv zu konnotieren. 
berücksichtigt: die Arbeit mit einem 'Infokoffer'.

Die vorangegangenen Kapitel haben versucht, wissenschaftlich darzulegen, warum ein solcher Koffer als sinnvolles didaktisches Werkzeug eingesetzt werden kann. Dennoch lässt sich Bildung und Integration nicht in seiner Gänze evaluieren, weil sich diese Werte nicht umfassend in Zahlen über alle Menschen erheben lassen. Empirische Forschung bedeutet in diesem Bereich, dass man auf ausgewählte spezielle Populationen und (quasi-) experimentelle Bedingungen zurückgreifen muss und dabei eine künstliche Gruppe kreieren müsste. Beispielsweise: Teilnehmer die höchstens fünf Jahre zur Schule gegangen sind, Rechtshänder sind, 17 Jahre in Deutschland leben und über den gleichen deutschen Wortschatz verfügen. Nichtsdestotrotz wird eine Integrationsbereitschaft gefordert, und dadurch politisch und sozioökonomisch Druck ausgeübt sich zu integrieren, ungeachtet der Lebenswelt der Menschen und plakativ ausgedrückt: ohne Rücksicht auf inter- und intraindividuelle Unterschiede. Auch wenn eine empirische Evaluation von Bildung, Persönlichkeitsentwicklung und Integration, insbesondere bei vor langer Zeit migrierten und sozial zurückgezogenen Menschen schwer möglich zu sein scheint, besteht ein Bedarf daran um eine evidente Basis für eine erforderliche neue gesellschaftliche Haltung zu etablieren. AUERNHEIMER bringt die Überlegungen 2007 auf den Punkt:

\footnotetext{
Grundlegend ist die Annahme „der unhintergehbaren Eigenart differenter Lebensweisen und Wissens- und Denkformen“ (S. 49), die aber „kulturell bedingt und historisch veränderlich sind“ (S. 55). Das heißt, ebenso wie Vorstellungen vom „Wesen“ der Frau verworfen werden, so auch solche von fixen ethnischen Eigenheiten. Prengel wendet sich daher auch gegen "binäre Strukturen“ (wie männlich - weiblich, westlich - orientalisch, S. 181) und gegen die Suche nach einer angeblichen Authentizität (S. 183). Denn die Differenzen basieren auf Sozialisation und sozialer Konstruktion. (AUERNHEIMER 2007: 182).
}

Um eine solche Haltung gesamtgesellschaftlich fördern zu können, bedarf es selbstredend Konzepten die dies sozialisations-, alters- und kulturbedingt berücksichtigen. Eines dieser Konzepte kann der dargestellte 'Infokoffer' als praxisnahes Beispiel sein. Ein regressiver Blick auf die Entwicklung und Durchführung des ersten 'Infokoffers' zeigt, dass gewissermaßen bereits im Sinne der Grounded Theory ein Konzept entwickelt wurde, dass nun in dieser Arbeit erfasst und damit wissenschaftlich beschrieben wurde. Bei der Entwicklung dieses Projekts stand die Pragmatik für die alltägliche Anwendung im Vordergrund, die für diese Arbeit auch relevant ist - jedoch wurden nun die wissenschaftlichen 
Hintergründe beleuchtet. Da es mit Zuhilfenahme der Grounded Theory auch möglich ist Hypothesen zu entwickeln, können hier bereits Ergebnisse dieser Hypothesen dokumentiert werden. Diese wiederum führen in einem weiteren Schritt zum folgenden Kapitel, dem übergeordneten Resultat einer didaktischen Methode: 'Arbeiten mit dem 'Infokoffer'.

\section{2. 'Infokoffer' als mögliches didaktisches Werkzeug}

Der im Textverlauf vorgestellte Infokoffer kann als Beispiel für eine didaktische Herangehensweise dienen, z.B. speziell im Einsatz bei alten Migranten, und kann darüber hinaus für die daraus entstandenen facettenreichen Projekte als Werkzeug dienen. Damit kann gezeigt werden, dass Lernen auf diesem Weg funktioniert und die oben genannten Forderungen als erfüllt gelten können, wenn sich des richtigen Organums bedient wird. Der Erfolg des ursprünglichen Infokoffers ist unumstritten, und nach den vorliegenden Ausführungen lohnt sich eine Erprobung des Infokoffers für ältere türkischstämmige Migranten oder ältere AussiedlerInnen ${ }^{70}$.

Insofern sind die aus den Inforeihen mit dem "Informationskoffer" entstandenen Projekte Beispiele für gelungene didaktische Umsetzung, die die Nachhaltigkeit und Motivation beziehungsweise Persönlichkeitsveränderung bei den teilnehmenden alten Migranten darstellen. Diese sind beispielsweise auf der Webseite $^{71}$ der Inforeihe dokumentiert. Es ergaben sich nach Durchführung der Inforeihe zum Beispiel von ehemaligen Teilnehmern initiierte Erste-Hilfe-Kurse, Gymnastikgruppen und vor allem ein selbstinitiierter Deutschkurs. Somit kann man zusammenfassen, dass mit der 'richtigen' didaktischen Methode wie es der Infokoffer sein könnte, eine Grundlage geschaffen werden kann, um Gesundheit und Lebensqualität zu verbessern. Dies geschieht nicht zuletzt durch Erhöhung der Ich-Stärke, Zuwachs bei individueller und biografisch angepasster Bildung und Wissensvermittlung und äußert sich somit in einer Kapitalerhöhung der folgenden drei Kapitalformen:

70 Der Vollständigkeit halber muss an dieser Stelle erwähnt werden, dass - wenn nicht explizit angeführt - aus pragmatischen Gründen auf die maskuline Form der Nomen zurückgegriffen wurde, damit jedoch keinesfalls genderspezifische Diskriminierung zum Ausdruck gebracht werden soll.

71 http://www.aelter-werden-in.de//index.php, Letzter Zugriff 15.06.2011 


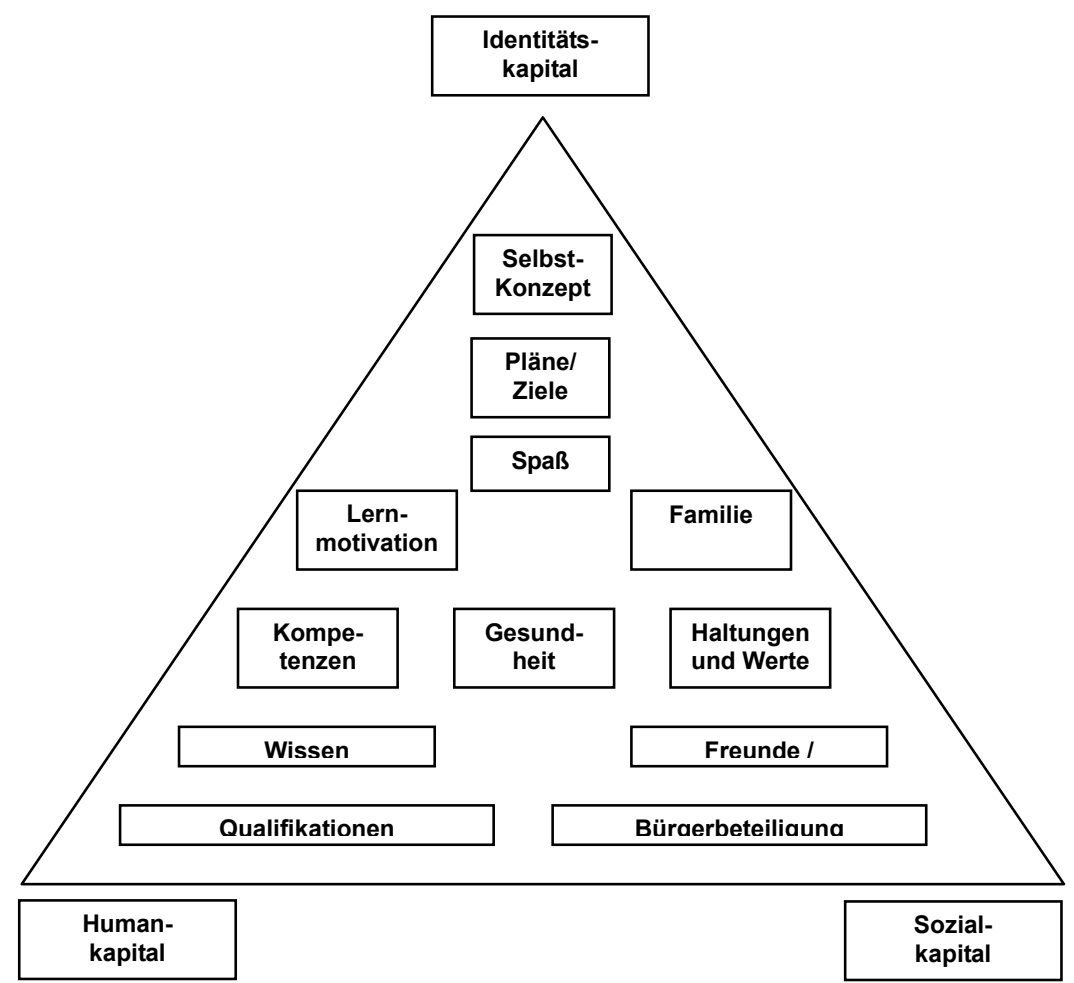

Abb. 5 Quelle: NoLDA, Sigrid (2008: 127)

AUERNHEIMER spricht für den interkulturellen Dialog 2007 einige unterstützenswerte Empfehlungen aus die Parallelen $\mathrm{zu}$ der unter $\mathrm{C} .1$ aufgeführten Aspekten aufweisen und damit die Basis für den Infokoffer stellen:

- Keine Verteidigungshaltung und keine missionarische Haltung einnehmen! Misstrauen ablegen (BoHM 1998)!

- Person und „Sache“ trennen! Das heißt, die Achtung des Gesprächspartners nicht von der Bewertung des fremden Normensystems oder Weltbilds abhängig machen!

- Keine falschen Wertmaßstäbe anlegen! Nicht die Ideale der eigenen Kultur mit der befremdlichen sozialen Realität der anderen vergleichen!

- Keine Glaubenskriege führen, sondern Lösungen für die jeweilige Situation, den jeweiligen Lebensbereich suchen! Eventuell einen dritten Weg beschreiten!

- Erfahrungen von Diskriminierung in Rechnung stellen!

- Die (frühere) Funktionalität vieler tradierter Muster für die gesellschaftliche Reproduktion berücksichtigen!

- Den Kampf um kulturelle Identität anerkennen, aber Individualrechte verteidigen! (AUERNHEIMER 2007: 141)

Alle diese Punkte gelten sowohl für 'normale' als auch für interkulturelle Dialoge. Insbesondere jedoch der Aspekt der Erfahrung von Diskriminierung (oder auch nur die Angst davor) ist vor dem Hintergrund der neurobiologisch erläuterten Blockaden und des Folgestresses zu unterstreichen. Aber auch den anderen Aspekten könnten beispielsweise mit einem Projekt wie dem Infokoffer Rechnung getragen werden. Die Forderung der Anerkennung der Verschiedenheiten der Kulturen und Menschen ist auch in der Integrationspädagogik nicht neu und lässt 
sich mit AUERNHEIMER unter Rückbezug auf PRENGEL auf die vorliegende Arbeit problemfrei übertragen:

Die Autorin [Annedore Prengel - Anm. I.Ö.], die von der Integrationspädagogik, also der Arbeit mit Behinderten herkommt, und sich zugleich in den Diskurs um Feministische Pädagogik eingemischt hat, ist bemüht, aus diesen beiden Diskursen, ergänzt um die Interkulturelle Pädagogik, ein neues Denken von Differenz und eine neue Art des Umgangs mit Differenzen zu begründen. Zentral geht es ihr darum, die traditionell mit Differenzen verquickten Rangordnungsvorstellungen aufzubrechen, einen „egalitären Differenzbegriff“ zu etablieren. Differenzen sollen anerkannt, ernst genommen werden, aber ohne die üblichen abwertenden Konnotationen. Postuliert wird die "intersubjektive Anerkennung zwischen gleichberechtigt Verschiedenen" [Hervorhebung I.Ö.] (Prengel 1995: 62).

AUERNHEIMER geht sogar noch weiter und nennt die sogenannte „Pädagogik der Vielfalt", die mit ihren Zielen wie Selbstachtung und Anerkennung der Anderen, vor allem aber auch Neugier für das Andere (die im Sinne der unter „Neurodidaktik“ vorgestellten Überlegungen zum freiwilligen, lustvollen Lernen durch Neugier führen kann) argumentiert:

Die „Pädagogik der Vielfalt“ hat zum Ziel: Selbstachtung und Anerkennung der anderen, Neugier für das Andere, „Übergänge“, die Erfahrung gegenseitigen Lernens, Aufmerksamkeit für die Vielfalt der Differenzen und ihre Überschneidungen, auch für die sich kreuzenden Dominanzverhältnisse (z.B. Mädchen mit deutschem Pass - Jungen mit Ausländerstatus) und außerdem Aufmerksamkeit für gesellschaftliche und ökonomische Bedingungen (S. 184ff.). (AUERNHEIMER 2007: 135)

Eine Möglichkeit dieses freiwillige, lustvolle Lernen zu fördern, kann der vielfach erwähnte Infokoffer darstellen. Im Folgenden wird abschließend erläutert, wie sich dieser als weiterentwickelter Baustein in den bestehenden Göttinger Katalog Didaktischer Modelle einfügen lassen könnte, in dessen Rahmen diese Arbeit unter anderem entstanden ist. Der beschriebene Methodenvorschlag orientiert sich an dem Muster und den 20 bereits verfassten Modellen aus dem „Kleinen Handbuch Didaktischer Modelle“ von Karl-Heinz Flechsig (1996). Es ist festzustellen, dass mithilfe des Infokoffers zahlreiche Methoden aus dem Göttinger Katalog Didaktischer Modelle (GKDM) integriert und erweitert werden können.

Entsprechend den Vorgaben des Göttinger Kataloges didaktischer Modelle von FLECHSIG $(1983,2003)$ könnte die Arbeit mit einem Infokoffer wie folgt als Methode eingearbeitet werden: 


\section{Infokoffer}

Anknüpfungspunkte

Alltagserfahrung:

Entdecken wenig oder unbekannter Situationen, Lebenskontexte, neuer sprachlicher Begriffe. Beginn neuer Erfahrungen und Orientierungen; Erlebnis von Neuem auf verschiedenen Wahrnehmungs- und Verarbeitungsebenen.

Historische Beispiele:

z.B. orthopädische Sammlung des Pathologen Dr. Karl Anton Portele, Musterkoffer eines englischen Orthopädiemechanikers aus der Zeit um 1900; 1866 benutzte Levi Strauss, der Vater der berühmten Blue Jeans, einen Musterkoffer als reisender Händler.

Bezeichnung, englisch: presentation case.

Bezeichnung, französisch: présentation cas.

Bezeichnung, türkisch: tanıtım valizi.

\section{Didaktische Prinzipien}

Lernen durch Erfahrung und Umgang, Ganzheitliches Lernen, Orientierendes

Lernen, Beiläufiges Lernen, Lernen durch/mit Medien, Thematisch orientiertes

Lernen, Lernen durch wechselseitigen Erfahrungsaustausch, Rollenspiele, Simulationen, Show-and-Tell.

\section{Phasen und Handlungsabläufe}

Vorbereitungsphase - Auseinandersetzungsphase - Reflexionsphase - ggf. Übungsphase - Durchführungsphase - Bewertungsphase.

\section{Elemente und deren Eigenschaften}

Lerner:

sollte selbständig erkunden können, Interesse finden können, auf Basis einer Eigenmotivation teilnehmen, zu kooperativer Aktion fähig sein.

\section{Moderator:}

muss Gruppe empathisch steuern können, Gruppengespräche moderieren, individuelle Anliegen mit der Gruppe vermitteln können und Ergebnisse integrieren.

Kontaktperson/en im Feld:

vermittelt Erfahrungsmöglichkeiten und steht als Informator zur Verfügung.

Erkundungsfeld/Aufgaben/Material:

muss organisiert bzw. passend zu der Gruppe ausgewählt werden, muss soz./kult. Hintergrund der 'Lerner` berücksichtigen.

\section{Geltungsbereich}

Institutionen: Alle.

Lerner: Alle Gruppen von Personen.

Phasen von Lehrgängen:

Lehrgangsunabhängig, auch in jeder Phase von Lehrgängen, insbesondere zur Orientierungs- und Eingewöhnungsphase, auch bei neuen Inhaltsbereichen, Lehrgangsbegleitend.

Varianten und Kombinationen

Einsatz verschiedener Medien, Exkursionen/Erkundung, Vorführung, Demonstration, Spiele, Selbsterfahrung, Methode nach FREIRE.

Ein weiterer Vorteil dieser Methodik ist, dass sie zumindest in gewissem Maße eine Evaluation zulässt, in einem Bereich, der schwer messbar ist. Da neu gewonnene Bildung und neu erworbenes Wissen schwer messbar sind, können 
jedoch die Bereiche, auf die sich das neu erworbene Wissen (und auch die Art des neu erworbenen Wissens) auswirkt, im Sinne von Erhebungen zur Lebenszufriedenheit (z.B. in Form von qualitativen Forschungsmethoden wie qualitativen Interviews) evaluieren lassen. In einem weiteren Schritt lässt sich der Infokoffer auch auf andere Zielgruppen anwenden als nur auf (alte) Migranten, eine Zielgruppe könnten beispielsweise auch Kindergärten sein - eben all jene Bereiche in denen Lernen im weitesten oder engeren Sinn auf verschiedene Arten als Thematik relevant ist. Nicht nur der Zielgruppe entsprechend (Jugendliche benötigen andere Medien als alte Migranten) können die Medien im Infokoffer erweitert und ausgetauscht werden, auch soll dem technischen und forschungstechnischen Fortschritt anhand des Bausteinsystems des Koffers Rechnung getragen und damit die Aktualisierbarkeit gewährleistet werden können.

\section{Ausblick}

Ein weiteres Ziel der vorliegenden Arbeit ist es, Anreiz zu weiterführender, vertiefender Forschung zu bieten. Neben den zuvor angeführten Überlegungen haben sich in der psychotherapeutischen, praktischen klinischen Tätigkeit viele weitere Aspekte ergeben, die im Sinne der Grounded Theory über die Jahre erfahren, durchdacht, erprobt oder auch nur konzeptionalisiert wurden. Als Beispiel für die Beschäftigung mit den Themen Therapie, Trauma, Stress, Neurobiologie, Migration, Kultur und aus der praktischen Tätigkeit heraus auch dem spezifischen Thema Boderline, entstand über die Jahre die Idee der 'soziokulturellen Boderlinhaftigkeit', die im Anschluss kurz vorgestellt wird. Ein weiteres Beispiel aus der therapeutischen Praxis ist ein ressourcenorientiertes, traumatherapeutisches Gruppeninterventionsverfahren für Migranten und Flüchtlinge, dass in der Behandlung der Stressfolgestörung gleichzeitig den Akkulturationsstress mit berücksichtigt, sprachreduziert Stabilisierungsarbeit leistet und auch die Biografie und Zuwanderungsgeschichte der Menschen mitbeachtet (vgl. ÖZKAN 2010). Das Manual dafür liegt bereits bei Verlagen vor.

Die Überlegungen zur Idee der Aspekte einer 'sozio-kulturellen Boderlinehaftigkeit' hingegen sind noch nicht derartig ausgebildet, sollen im Folgenden aber dargestellt werden und genau wie obige Methodik Anreiz zu vertiefender Forschung liefern.

Der Grundgedanke der Idee lässt sich ebenfalls auf Stress zurückführen 
und hat folgende Überlegung als Fundament: Allein die Vorstellung das Ziel der Migration nicht erreichen zu können, nämlich für die Generation der Ersteingewanderten die Rückkehr in das Ursprungsland und für die Folgegenerationen das Gefühl, im Zielland nicht akzeptiert zu werden, kann zu unkontrollierbaren neuroendokrinen Reaktionen führen, ganz im Sinne der angeführten Thesen von HÜTHER oder des 'antizipierten Lernens' FLECHSIGS. Addiert man zu dieser Imagination noch die Angst vor Rassismus, Diskrimiernung in der Fremde oder auch für die Generation der Ersteingewanderten die Befürchtung bei ihrer Rückkehr allein in der veränderten Heimat zu sein, keine soziale Sicherung zu haben und ähnliches, scheint eine psychische Stressfolgestöung unumgänglich. Ein Beispiel aus den Medien ist FERIDUN ZAIMOGLU, ein türkisstämmiger Schriftsteller, der bei seinen Lesungen und öffentlichen Auftritten konstatierte, dass er als ein Ausländer immer doppelt besser sein müsse als ein Deutscher, um sich behaupten zu können. Die in der Einleitung von Bukow zitierte Problematik ist somit nach wie vor aktuell und scheint für die folgenden Generationen ebenso gültig zu sein, wie für die Ersteingewanderten, da diese sich (bereits den potentiellen) Diskriminierungen entgegensetzen und beweisen möchten. Sie möchten und müssen ihre Identität zwischen den Welten zwischen denen sie wandeln auf nochmals andere Weise finden und bestätigt finden, als das Nicht-Migranten tun. Mit diesem Grenzgang zwischen den (Identitäts-)Welten ist ein hoher Druck verbunden und damit Stress. Die antizipierte Diskriminierung ist bereits Persönlichkeitseigenschaft, weil die nachfolgenden Generationen bereits derartig enkulturiert werden (siehe hierzu auch die Thematik der transgenerationalen ${ }^{72}$ Weitergabe von z.B. Traumata als Stressfolgestörung oder auch die biologische Weitergabe von Stress ohne, dass sich die Genetik verändert: Epigenese ${ }^{73}$ ). Das wiederum führt zu ambivalenten Verhaltensweisen innerhalb der eigenen Kultur und der dominanten kulturellen Umgebung - individuell belastend und häufig nicht funktional. Mit z.B. Überidentifikation an alt tradierten Symbolen, Helden, Riten wie sie das

72 Vgl. beispielsweise Gabriele RosenTHAL: Transgenerationale Folgen von Verfolgung und Täterschaft. Familien von Shoah-Überlebenden und von Nazi-Tätern. In: StreeckFischer/Sachsse/Özkan (Hg.) Körper - Seele - Trauma. Biologie, Klinik und Praxis. Göttingen 2002.

73 Vgl. beispielsweise Joachim BAUER: Das Gedächntis des Körpers: Wie Beziehungen und Lebensstile unsere Gene steuern. Frankfurt 2007. 
Eisbergmodell $^{74}$ der Kulturen (zur Zeit der Veröffentlichung von einem unbekannten Verfasser) darstellt, erfolgt jedoch eine Selbststereotypisierung:

\title{
Eisbergmodell
}

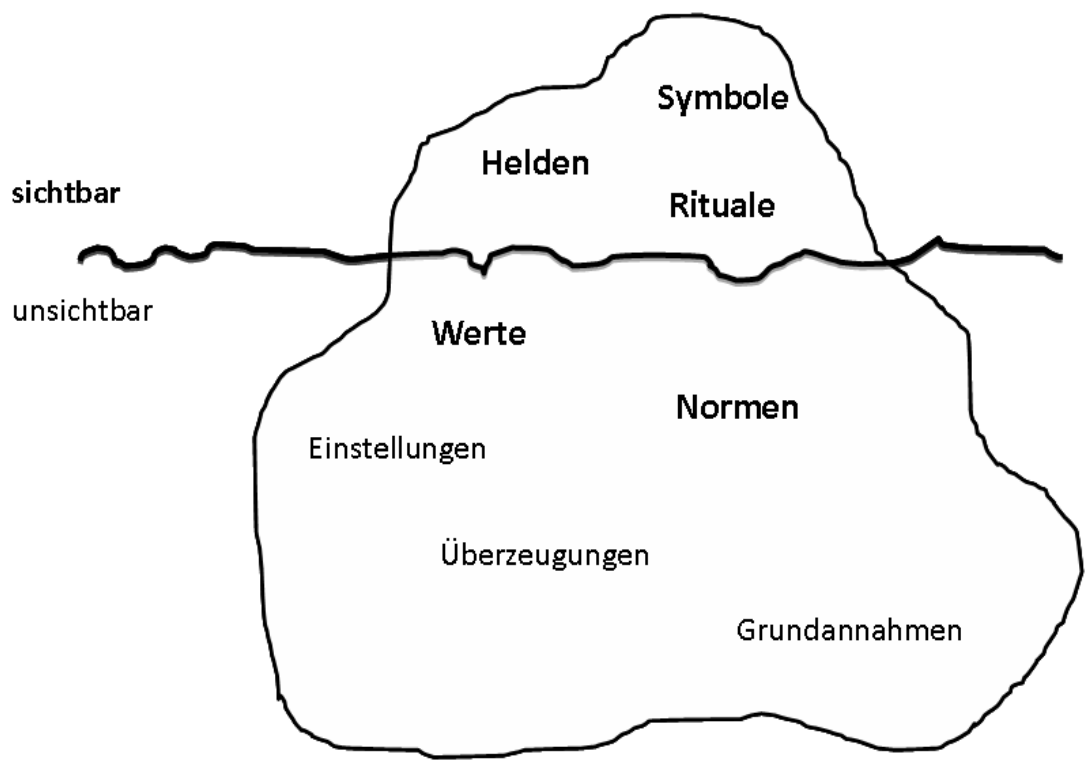

Abb. 6 'Eisberg der Kultur'

Diese Selbststerotypisierung kann jedoch im Alltag zu enormen Spannungen führen und als Stressor auftreten:

\begin{abstract}
Eine stigmatisierte Person hält sich zwar für ein „menschliches Wesen wie jeder andere“, erfährt aber gleichzeitig, daß sie von Nichtstigmatisierten, nicht als gleichberechtigtes menschliches Wesen akzeptiert wird. (vgl. Goffman [1963] 1967: 16). Die stigmatisierte Person kennt ihr Stigma und weiß, welche Attribute ihr von den Angehörigen der Mehrheitsgesellschaft zugeschrieben werden. Sie muß ständig ihre Eigenschaften problematisieren und sich fragen, inwieweit sie den stereotypen Vorstellungen über Ausländer entspricht. Vor allem muß sie sich und anderen beweisen, daß sie nicht die negativen Eigenschaften aufweist, die man ihr zuschreibt. Unter diesen Umständen ist es schwierig, ein positives Selbstwertgefühl und Selbstbewußtsein zu entwickeln.
\end{abstract}

(KEIM 2003: 118f)

Die Individuuen bewegen sich also zwischen den Polen der Selbststereotypisierung der tradierten kulturellen Identität und können eine sich auflehnende Haltung gegenüber der dominanten Kultur entwickeln, die als vermeintliche diskrimierende Bedrohung gesehen werden kann. Um sich in dieser Polarität (ver-)orten zu können, findet ein Grenzgang zwischen Überidealisierung der 'eigenen' Kultur (beispielsweise tragen junge Kinder von ehemaligen Gastarbeitern mit Stolz eine Halskette mit den Symbolen der Türkei, ohne jemals

74 Modified from Gary R. WEAVER, "Understanding and Coping with Cross-cultural Adjustment Stress" in Gary R. Weaver, editor, Culture, Communication and Conflict: Readings in Intercultural Relations, second edition (Simon \& Schuster Publishing, 1998) 
da gewesen zu sein) und der für bedrohlich wahrgenommenen, fremden, dominierenden Kultur statt, deren beiden Anteile die Menschen jedoch in sich tragen. Gerade die erwähnten Adoleszenten bewegen sich stark an dieser Grenze, da sie sich in ihrer Entwicklung noch stärker in der Thematik der Selbstidentifikation und Identitätsbildung befinden, als das möglicherweise ältere Menschen tun. Grenzgänger sind in der Regel in der klinischen Psychologie/Psychiatrie als Borderliner bekannt.

Nach den Leitlinien der Deutschen Gesellschaft für Psychiatrie, Psychotherapie und Nervenheilkunde (DGPPN), der Deutschen Gesellschaft für Psychosomatische Medizin und Ärztliche Psychotherapie (DGPM), dem Deutschen Kollegium für Psychosomatische Medizin (DKPM), der Deutschen Gesellschaft für Psychologie (DGP), der Fachgruppe Klinische Psychologie und Psychotherapie und der Deutschen Gesellschaft für Kinder- und Jugendpsychiatrie und -psychotherapie (DGKJP) wird die Borderline-Persönlichkeitsstörung wie folgt beschrieben $^{75}$ :

\section{DSM-IV (301.83) Borderline-Persönlichkeitsstörung}

Ein tiefgreifendes Muster von Instabilität in zwischenmenschlichen Beziehungen, im Selbstbild und in den Affekten sowie von deutlicher Impulsivität. Der Beginn liegt im frühen Erwachsenenalter und manifestiert sich in den verschiedenen Lebensbereichen. Mindestens fünf der folgenden Kriterien müssen erfüllt sein:

1. verzweifeltes Bemühen, tatsächliches oder vermutetes Verlassenwerden zu vermeiden. Beachte: Hier werden keine suizidalen oder selbstverletzenden Handlungen berücksichtigt, die in Kriterium 5 enthalten sind.

2. Ein Muster instabiler, aber intensiver zwischenmenschlicher Beziehungen, das durch einen Wechsel zwischen den Extremen der Idealisierung und Entwertung gekennzeichnet ist.

3. Identitätsstörung: ausgeprägte und andauernde Instabilität des Selbstbildes oder der Selbstwahrnehmung.

4. Impulsivität in mindestens zwei potentiell selbstschädigenden Bereichen (Geldausgaben, Sexualität, Substanzmissbrauch, rücksichtsloses Fahren, "Fressanfälle"). Beachte: Hier werden keine suizidalen oder selbstverletzenden Handlungen berücksichtigt, die in Kriterium 5 enthalten sind.

5. Wiederholte suizidale Handlungen, Selbstmordandeutungen oder -drohungen oder Selbstverletzungsverhalten.

6. Affektive Instabilität infolge einer ausgeprägten Reaktivität der Stimmung (z. B. hochgradige episodische Dysphorie, Reizbarkeit oder Angst, wobei diese Verstimmungen gewöhnlich einige Stunden und nur selten mehr als einige Tage andauern).

7. Chronische Gefühle von Leere.

8. Unangemessene, heftige Wut oder Schwierigkeiten, die Wut zu kontrollieren (z. B. häufige Wutausbrüche, andauernde Wut, wiederholte körperliche Auseinandersetzungen).

9. Vorübergehende, durch Belastungen ausgelöste paranoide Vorstellungen oder schwere dissoziative Symptome.

75 AWMF online. Arbeitsgemeinschaft der Wissenschaftlichen Medizinischen Fachgesellschaften. http://www.awmf.org/uploads/tx_szleitlinien/038-015_S2_Persoenlichkeitsstoerungen_05-

2008_05-2013.pdf, 14.06.2011. 
Im Fall von Boderlinepersönlichkeitsstörungen zeigen Menschen ein verzweifeltes Bemühen zu verhindern, tatsächlich oder vermeintlich verlassen zu werden. Zwischenmenschliche Beziehungen sind höchst problematisch und die 'Boderliner' pendeln zwischen Idealisierung und Entwertung dieser Bindungen und der entsprechenenden Beziehungspartner. Meist haben sie zudem ein instabiles Selbstbild.

Die dabei entstehenden vielschichtigen kulturellen oder individuellen Identitäten werden in der Sozialwissenschaft als zusammengesetzt und vieldeutig, als "Patchwork-Identities" oder als "multiple Identitäten "bezeichnet (KEUPP 1998). STUART HALL spricht von der Konstruktion von kulturellen Identitäten in unterschiedlichen Kontexten (HALL 1994,1997). Die Identität des Individuums im Sinne eines stabilen Selbst entwickelt sich dagegen in den frühen Beziehungen, ist aber auch kulturell gefärbt. Dieses Kern-Selbst erfordert oft enorme psychische Leistungen, um sich gleich zu fühlen inmitten von sich wandelnden äußeren Verhältnissen und neuen Teilidentitäten, die auch wieder zu integrieren sind (BOHLEBER 1992). (NADIG 2010: 59)

\section{In dem Patchwork eine Nicht-Ortung für sich zu finden, macht die Spannung}

aus, führt zum Gefühl der Nicht-Zugeörigkeit und wird von BHABHA mit Deplazierung und Entortung bezeichnet:

Die Begegnung mit Identität findet jeweils an einem Punkt statt, an dem etwas über den Rahmen des Bildes hinausgeht, dem Auge verborgen bleibt, dass Selbst als Ort der Identität und Autonomie entleert und - dies ist am wichtigsten - eine widerständige Spur zurücklässt, einen Fleck des Subjekts, ein Zeichen von Widerstand.

(BНАBHA 2000: 73)

\section{Weiterführend schreibt er:}

Sobald das "kulturelle" als Artikulation von De-plazierung und Entortung aufgefasst wird, ist es möglich, es als eine Anordnung von Macht, als negative, agonistisch an der Grenze zwischen Referenzrahmen und mentalem Rahmen konstruierte Transparenz zu identifizieren.

(BHABHA 2000: 169)

Und er führt knapp und dennoch ausführlich das Problem aus, das im Anschluss thematisiert wird:

Das Problem ist nicht ontologischer Art, bei der Differenzen sich aus einer stärker totalisierenden, transzendenten Identität ergeben, die entweder in der Vergangenheit oder in der Zukunft zu finden ist. Hybride Bindestrich-Bildungen (hybrid hyphenations) betonen die inkommensurablen Elemente - die hartnäckigen Klumpen - als die Basis kultureller Identifikationen. Es geht hier um die performative Natur differenzieller Identitäten: um die Regulierung und Verhandlung jener Räume, die sich beständig, kontingent, "nach außen öffnen", welche die Grenzen neu ziehen, die die Beschränktheit jedweder Forderung nach einem singulären oder autonomen Zeichen der Differenz - sei es nun Klasse, Geschlecht oder Rasse - offenbaren. Solche Zuweisungen sozialer Differenzen - bei denen die Differenz weder Eines noch das Andere, sondern etwas anderes daneben, da-zwischen ist, finden ihre Handlungsmacht in Form der "Zukunft", in der die Vergangenheit nicht ursprünglich und in der die Gegenwart nicht einfach ein Übergang ist. Es ist, wenn ich hier diesen Punkt noch etwas dehnen darf, eine zwischenmenschliche Zukunft, die zwischen den Ansprüchen der Vergangenheit und den Bedürfnissen der Gegenwart entsteht.

(BHABHA 2000: 327) 
Bei IRLE und SACHSSE (2005) konnten bei BorderlinePersönlichkeitsgestörten auf biologischer Ebene Assymmetrien im Hippocampusarreal mit Hilfe bildgebender Verfahren nachgewiesen werden. Interessant für weitere Forschung wäre nun, gerade mit neueren Methoden Menschen zu untersuchen, die mit dieser jahrzehntelangen, kulturellen Ambivalenz als Stressor in der Fremde leben und dabei zu prüfen, ob und wie sich dabei vergleichbare Assymmetrien entwickeln. Denn die Wanderung auf der Grenzlinie zwischen den Kulturen zeigt Symptome der Boderlinepersönlichkeitsstörung, sodass die Frage gestellt werden kann: Gibt es eine ‘sozio-kulturelle Borderlinehaftigkeit' bei Menschen z.B. mit Zuwanderungsgeschichte?

Falls dem so ist, stellt sich weiterhin als Ausblick die Frage, wie damit umgegangen werden kann.

LINEHAN (1993) erarbeitete ein Konzept für Personlichkeitsstörungen, das in den folgenden Jahren als Basis für Methoden der Behandlung von Menschen mit Stressfolgestörungen diente. Dabei geht es hauptsächlich darum, durch eine Beziehungsarbeit unter Berücksichtigung der Auswirkungen der Störung, eine für das Gegenüber stressreduzierende Haltung einzunehmen, die eine funktionale (i.S.v. förderliche) Veränderung des Verhaltens der gestörten Persönlichkeit sorgen soll.

Aus dem was LINEHAN (1993) 'wise mind' nennt, das sich ergibt aus Wissen/Erfahrung sowie Gefühlen, und dem was NADIG (2010) in der Dynamik der Begegnung den 'transkulturellen Begegungsraum' nennt, würde es für den gesellschaftlichen Umgang mit Migration bedeuten, dass man außer auf der individuellen Ebene, auch auf der gesamtgesellschaftlich-politischen Ebene in dem was gesagt wird, den Raum der 'Transkulturellen Achtsamkeit' betreten würde, wie folgende Grafik darstellt: 


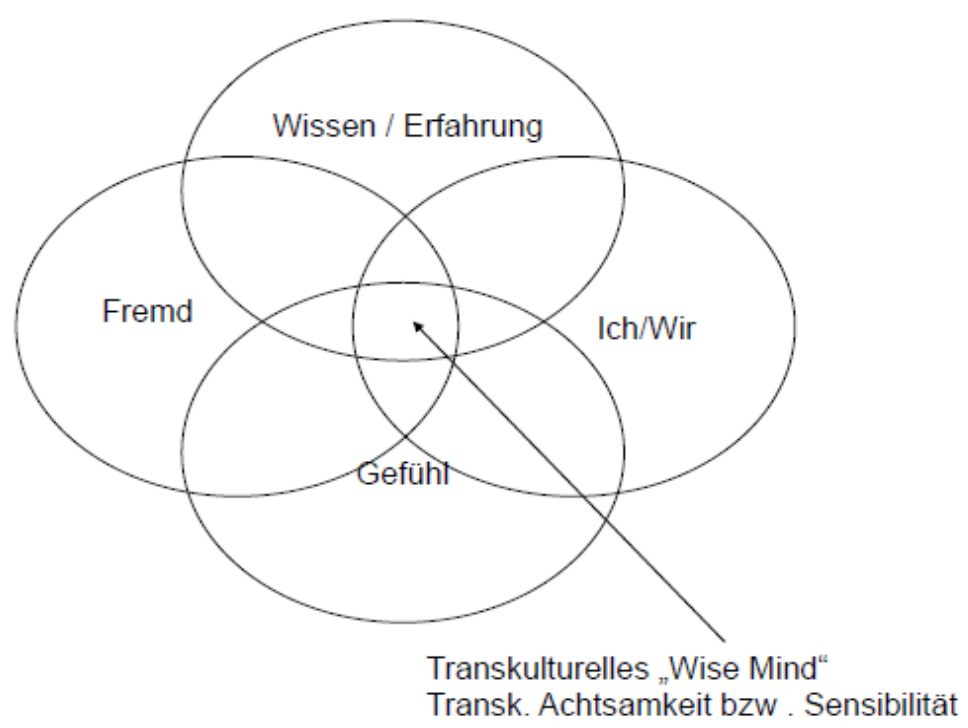

Abb. 7 'Transkulturelle Achtsamkeit' (Schnittgrafik LINNEHAN/NADIG) (ÖZKAN 2011)

Dieser Raum fordert eine Haltung, die nicht nur dem Wissen über andere Kulturen folgt, sondern auch von Gefühlen in der Begegnung geleitet ist und gleichzeitig aber der Individualität und der Identitätsbeibehaltung des Anderen Raum lässt. Entstehen kann diese Haltung beispielsweise durch einen nicht wertenden, beschreibenden, teilnehmenden und authentischen Umgang bzw. die Schaffung von Lebenswelten, die dann den Status einer Utopie verlieren. 


\section{Anhang}

\section{FLZ-Manual}

Die FLZM sind ein Fragebogen zur Erfassung der subjektiven Lebensqualität.

\section{Kurzbeschreibung}

Der Fragebogen besteht aus drei Teilen ("Modulen"):

- das Modul "Allgemeine Lebenszufriedenheit" enthält acht Items (acht relevante Lebensbereiche - siehe graphische Darstellung auf der nächsten Seite), die jeweils von der Testperson nach "subjektiver Zufriedenheit" und zusätzlich nach "subjektiver Wichtigkeit" beurteilt werden

- $\quad$ in dem Modul "Gesundheit" werden acht relevante Aspekte der Gesundheit nach "Zufriedenheit" und "Wichtigkeit" beurteilt

- das Modul "Magen-Darm-Aspekte" (Vorversion) spezifiziert acht gastroenterologische Aspekte der Gesundheit.

\section{Äußere Form}

Jedes Modul umfaßt eine DIN-A4-Seite und enthält Überschrift, Instruktion, Fragen nach Wichtigkeit (W) und Zufriedenheit (Z) für jedes Item mit jeweils fünf verbalisierten Antwortkategorien und Raum für eine Zusatzfrage. Das Modul "Allgemeine Lebenszufriedenheit" enthält zusätzlich eine Frage nach der globalen Lebenszufriedenheit.

\section{Testentwicklung}

Die Entwicklung der FLZ erstreckt sich über mehrere Phasen der Datenerhebung an großen Stichproben von gesunden und kranken Personen, in denen aufgrund von statistischen Analysen (Faktoren-, Itemanalysen, Gruppenvergleiche, Korrelationen) und aufgrund der Rückmeldung der Testpersonen Zahl und Formulierung der Items, Zahl und Verbalisierung der Antwortkategorien und Form der Instruktion modifiziert und optimiert wurde. Die FLZ nehmen inhaltliche Validität für sich in Anspruch. Test- und ItemKennwerte liegen vor. Die Module "Allgemeine Lebenszufriedenheit" und "Gesundheit" sind für den Bereich BRD (Ost und West) normiert.

\section{Anwendungsbereich}

Die Module "Allgemeine Lebenszufriedenheit" und "Gesundheit" der FLZ können zur Bestimmung der subjektiven Lebensqualität bei Patienten mit chronischen oder anderen schwerwiegenden Erkrankungen, bei gesunden Vergleichsstichproben und im Längsschnitt zur Beurteilung des Krankheitsverlaufs und der erwünschten und unerwünschten Wirkungen von therapeutischen Maßnahmen eingesetzt werden.

\section{Testanwendung}

Die FLZ können in Einzel- und in Gruppenuntersuchungen vorgegeben werden. Da der Fragebogen die Anweisung zum Ausfüllen enthält, ist nur darauf hinzuweisen, daß die Instruktion sorgfältig zu lesen und zu beachten sei. Je Modul ist eine Beantwortungszeit von 2-5 Minuten anzusetzen. Durchführungs- und Auswertungsobjektivität ist gegeben.

\section{Auswertung und Interpretation}


Die Wichtigkeit (W) und Zufriedenheit (Z) werden jeweils auf einer fünfstufigen Skala (Werte 1 bis 5) beurteilt.

Je Item (Lebensbereich, Aspekt der Gesundheit) können zwei Werte verwendet werden:

- $\quad$ die gewichtete Zufriedenheit $\left(\mathrm{W}^{*} \mathrm{Z}\right)$ nach der Formel ${ }^{1}$

$$
\mathrm{W} * \mathrm{Z}=(\mathrm{W}-1) *(\mathrm{Z} * 2-5) \text {, }
$$

die den Anteil des entsprechenden Lebensbereichs (bzw. Gesundheitsaspekts) an der globalen Lebenszufriedenheit darstellt (Skalenbereich: -12 bis +20)

- die Wichtigkeit (W) allein, als Maß für die subjektive Bedeutung eines Items für die Lebensqualität der Testperson; dieser Wert spiegelt (besonders im Längsschnitt) die Wirkung von Coping-Prozessen wider (Auf- bzw. Abwerten von Lebensbereichen). Je Modul beschreibt der Summenwert der $\mathrm{W}^{*} \mathrm{Z}$-Werte die globale Zufriedenheit mit dem entsprechenden Inhalt.

\section{Graphische Darstellung}

Die (transformierten) $\mathrm{W}^{*} \mathrm{Z}$-Werte der Items eines Moduls einer Testperson (oder die entsprechenden Mittelwerte einer Gruppe) können folgendermaßen dargestellt werden (hier "Allgemeine Zufriedenheit"):

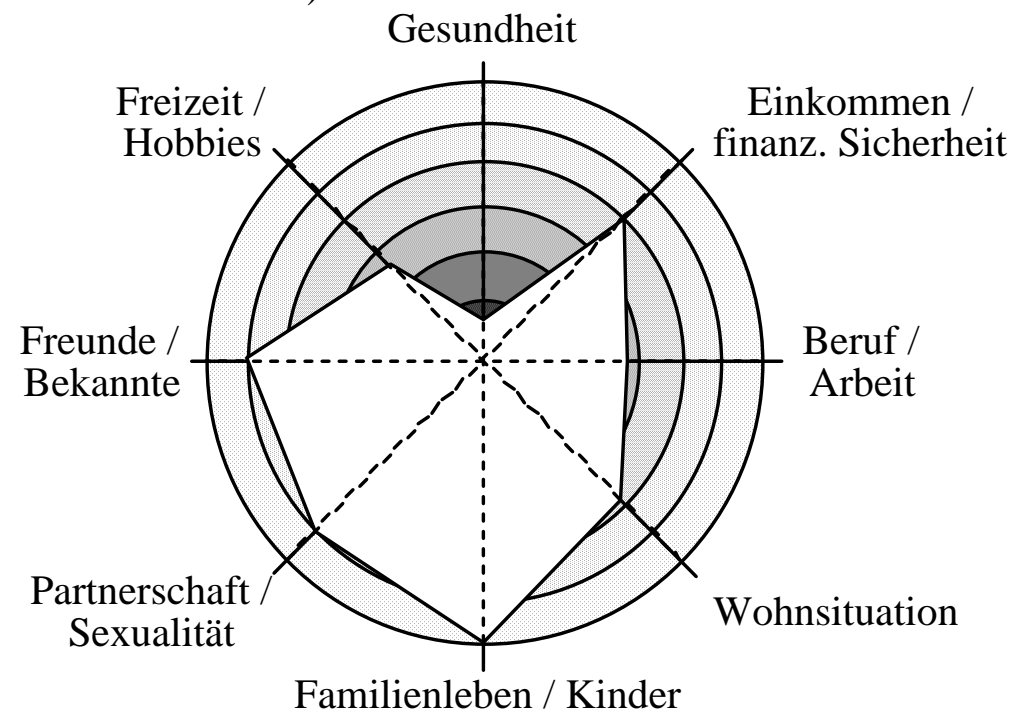

Der Abstand der Einzelpunkte vom Kreismittelpunkt repräsentiert den gewichteten Beitrag jedes Items zur Zufriedenheit insgesamt, die durch die Punkte aufgespannte Fläche die globale Lebensqualität.

- Die Rangreihe der resultierenden Werte entspricht nach Expertenrating der psychologischen Rangreihe der Begriffspaare Wichtigkeit/Zufriedenheit, nicht jedoch die Skalenabstände. Die W*ZWerte sind dementsprechend als Ordinalskala zu behandeln. 


\section{Literatur zu den FLZ}

Huber, D., Henrich, G. \& Herschbach, P.: Measuring the quality of life: A comparison between physically and mentally chronically ill patients and healthy persons. Pharmacopsychiatry 21, 453-455, 1988

Roder, J.D., Herschbach, P., Ritter, M., Kohn, M., Sellschopp, A. \& Siewert, J.R.: Lebensqualität nach Oesophagektomie. Deutsche Medizinische Wochenschrift 115, 570574, 1990

Roder, J.D., Herschbach, P. \& Siewert, J.R.: "Lebensqualität" nach Oesophagektomie beim Oesophaguskarzinom. Acta Chirurgica Austriaca 22, 48-50, 1990

Herschbach, P.: Möglichkeiten der Erfassung von Lebensqualität bei gastroenterologischen Patienten. In: Delbrück, H. (Hrsg.). Krebsnachsorge und Rehabilitation - Band 3. Magenkarzinom. W. Zuckschwerdt Verlag, München, 118-130, 1991

Herschbach, P. \& Henrich, G.: Der Fragebogen als methodischer Zugang zur Erfassung von "Lebensqualität" in der Onkologie. In: Schwarz, R., Bernhard, J., Flechtner, H., Küchler, Th. \& Hürny, Ch. (Hrsg.): Lebensqualität in der Onkologie. W. Zuckschwerdt Verlag, München, 34-46, 1991

Roder, J.D., Herschbach, P., Sellschopp, A. \& Siewert, J.R.: Quality-of-life assessment following oesophagectomy. Theoretical Surgery 6, 206-210, 1991

Henrich, G., Herschbach, P. \& von Rad, M.: "Lebensqualität" in den alten und neuen Bundesländern. Psychotherapie Psychosomatische Medizin Medizinische Psychologie 42, 31-32, 1992

Roder, J.D., Herschbach, P., Henrich, G., Nagel, M., Böttcher, K. \& Siewert, J.R.: Lebensqualität nach totaler Gastrektomie wegen Magenkarzinoms. Deutsche Medizinische Wochenschrift 7, 241-247, 1992

Herschbach, P., Henrich, G. \& Oberst, U.: Lebensqualität in der Nachsorge. Eine Evaluationsstudie in der Fachklinik für Onkologie und Lymphologie, Bad Wildungen-Reinhardshausen. Praxis der Klinischen Verhaltensmedizin und Rehabilitation 28, 241251, 1994

Henrich, G. \& Herschbach, P.: Fragen zur Lebenszufriedenheit (FLZ) - ein Gewichtungsmodell. In: Schwarz, R., Bernhard, J., Flechtner, H., Küchler, Th. \& Hürny, Ch. (Hrsg.): Lebensqualität in der Onkologie II. W. Zuckschwerdt Verlag, München, 77-93, 1995

Roder, J.D., Stein, H.J., Eckel, F., Herschbach, P., Henrich, G., Böttcher, K., Busch, R. \& Siewert, J.R.: Vergleich der Lebensqualität nach subtotaler und totaler Gastrektomie beim Magenkarzinom. Deutsche Medizinische Wochenschrift 121, 543-549, 1996

Zwei Publikationen mit den psychometrischen Kennwerten der FLZ und dem Vergleich von verschiedenen Personengruppen sind in Vorbereitung:

Henrich, G. \& Herschbach, P. (1996): Questions on Life Satisfaction (FLZ ${ }^{\mathrm{M}}$ ) -

Psychometric Properties of a New Questionnaire on Subjective Quality of Life.

Quality of Life Research, submitted 


\section{Auswertung mit dem Personal Computer (PC)}

Die computergestützte Auswertung der FLZ kann mit vielen der handelsüblichen StatistikProgrammen (z.B. SPSS, SAS oder BMDP) auf einem PC durchgeführt werden. Im folgenden sind die Rechenanweisungen für das in den Sozialwissenschaften häufig verwendeten Programmsystem SPSS (Statistical Package for Social Sciences, Base System) aufgelistet. Information über die Beschaffung ist von SPSS GmbH Software, Steinsdorfstraße 19, 80538 München (Telefon: 49-(0)89-228 3008, Telefax: 49-(0)89-228 5413) zu erhalten.

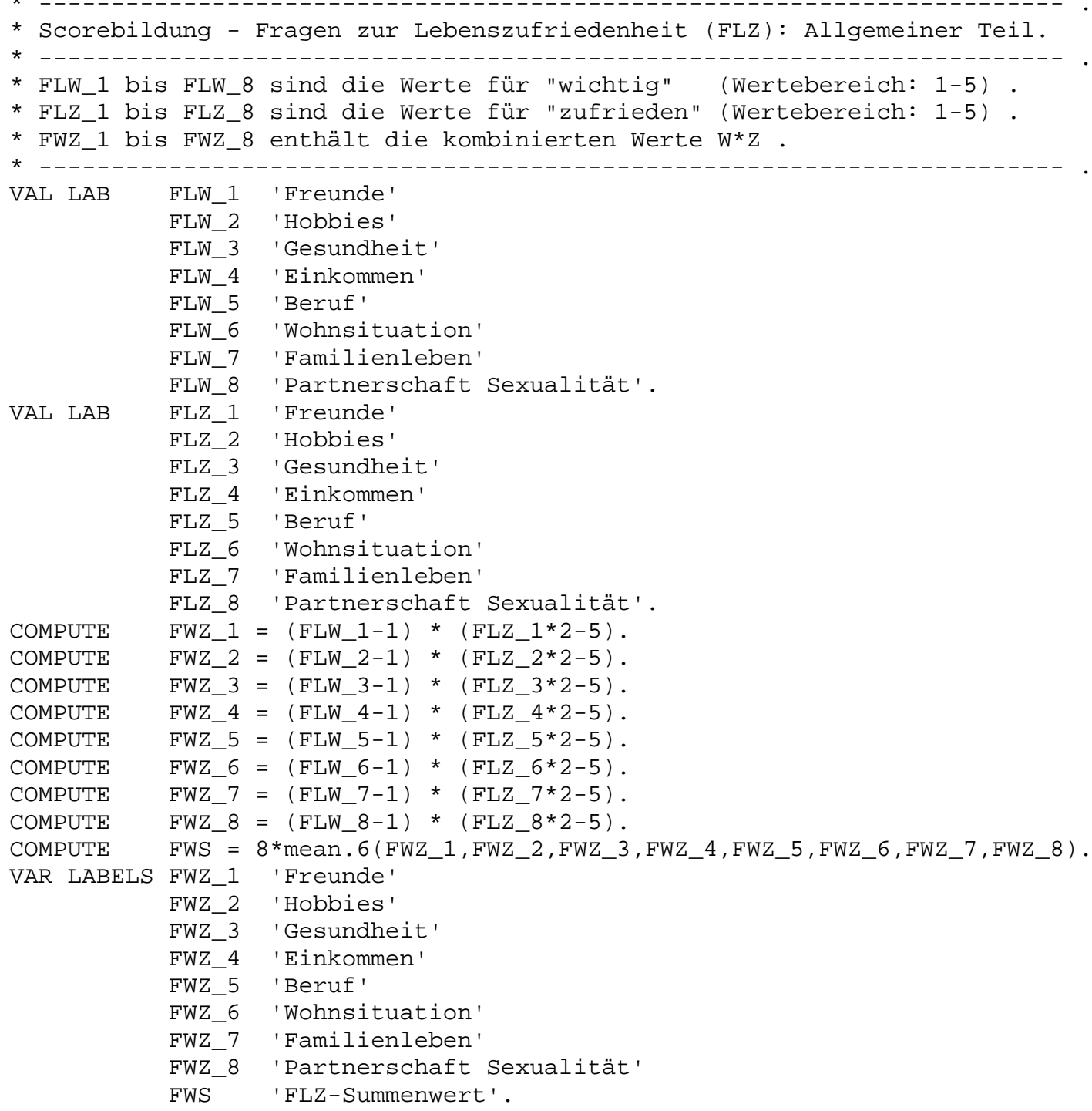




\section{Normdaten für die BRD-West und -Ost (alte und neue Bundesländer)}

\section{FLZ - Allgemeiner Teil: Normwerte BRD-West}

\begin{tabular}{|l|r|r|r|r|r|}
\hline Erhebung 1991 & \multicolumn{1}{c}{ Mean } & \multicolumn{1}{c|}{ SD } & \multicolumn{1}{c|}{ Min } & Max & N \\
\hline Freunde / Bekannte & 7,50 & 5,45 & -12 & 20 & 1917 \\
\hline Freizeit / Hobbies & 6,51 & 5,51 & -12 & 20 & 1915 \\
\hline Gesundheit & 8,64 & 6,89 & -12 & 20 & 1972 \\
\hline Einkommen / finanz. Sicherheit & 6,60 & 6,22 & -12 & 20 & 1969 \\
\hline Beruf / Arbeit & 6,35 & 6,27 & -12 & 20 & 1889 \\
\hline Wohnsituation & 8,74 & 6,55 & -12 & 20 & 1923 \\
\hline Familie / Kinder & 10,13 & 7,24 & -12 & 20 & 1920 \\
\hline Partnerschaft / Sexualität & 8,44 & 7,12 & -12 & 20 & 1931 \\
\hline FLZ-A-Summenwert & $\mathbf{6 2 , 8 2}$ & $\mathbf{3 4 , 6 1}$ & $\mathbf{- 6 0}$ & $\mathbf{1 6 0}$ & $\mathbf{1 9 4 5}$ \\
\hline
\end{tabular}

\begin{tabular}{|c|c|c|c|c|c|}
\hline Erhebung 1994*) & Mean & SD & Min & $\operatorname{Max}$ & $\mathbf{N}$ \\
\hline Freunde / Bekannte & 8,33 & 6,51 & -12 & 20 & 1688 \\
\hline Freizeit / Hobbies & 6,73 & 6,33 & -12 & 20 & 1687 \\
\hline Gesundheit & 8,35 & 7,55 & -12 & 20 & 1693 \\
\hline Einkommen / finanz. Sicherheit & 7,18 & 7,07 & -12 & 20 & 1690 \\
\hline Beruf / Arbeit & 6,06 & 7,04 & -12 & 20 & 1640 \\
\hline Wohnsituation & 8,67 & 6,31 & -12 & 20 & 1687 \\
\hline Familie / Kinder & 9,80 & 7,04 & -12 & 20 & 1678 \\
\hline Partnerschaft / Sexualität & 8,07 & 7,83 & -12 & 20 & 1672 \\
\hline FLZ-A-Summenwert & 63,21 & 37,79 & -64 & 160 & 1688 \\
\hline
\end{tabular}

\section{FLZ - Allgemeiner Teil: Normwerte BRD-Ost}

\begin{tabular}{|l|r|r|r|r|r|}
\hline Erhebung 1991 & \multicolumn{1}{c}{ Mean } & \multicolumn{1}{c}{ SD } & Min & Max & $\mathbf{N}$ \\
\hline Freunde / Bekannte & 7,52 & 5,59 & -9 & 20 & 991 \\
\hline Freizeit / Hobbies & 4,42 & 5,60 & -12 & 20 & 983 \\
\hline Gesundheit & 7,37 & 8,44 & -12 & 20 & 989 \\
\hline Einkommen / finanz. Sicherheit & $-0,48$ & 7,80 & -12 & 20 & 989 \\
\hline Beruf / Arbeit & 2,03 & 8,56 & -12 & 20 & 933 \\
\hline Wohnsituation & 6,51 & 7,57 & -12 & 20 & 991 \\
\hline Familie / Kinder & 11,17 & 7,04 & -12 & 20 & 982 \\
\hline Partnerschaft / Sexualität & 9,12 & 7,16 & -12 & 20 & 958 \\
\hline FLZ-A-Summenwert & 47,60 & 31,50 & -36 & 150 & 991 \\
\hline
\end{tabular}

* ) Normwerte getrennt nach Alter und Geschlecht siehe Anhang A 


\begin{tabular}{|l|r|r|r|r|r|}
\hline Erhebung 1994 & \multicolumn{1}{c}{ Mean } & \multicolumn{1}{c|}{ SD } & Min & Max & N \\
\hline Freunde / Bekannte & 7,05 & 5,42 & -9 & 20 & 847 \\
\hline Freizeit / Hobbies & 4,60 & 5,66 & -12 & 20 & 841 \\
\hline Gesundheit & 6,90 & 7,25 & -12 & 20 & 847 \\
\hline Einkommen / finanz. Sicherheit & 3,74 & 7,39 & -12 & 20 & 846 \\
\hline Beruf / Arbeit & 3,05 & 7,79 & -12 & 20 & 820 \\
\hline Wohnsituation & 6,98 & 6,56 & -12 & 20 & 844 \\
\hline Familie / Kinder & 10,00 & 6,52 & -12 & 20 & 840 \\
\hline Partnerschaft / Sexualität & 7,26 & 7,08 & -12 & 20 & 836 \\
\hline FLZ-A-Summenwert & $\mathbf{4 9 , 2 2}$ & $\mathbf{3 2 , 9 2}$ & $\mathbf{- 5 4}$ & $\mathbf{1 6 0}$ & $\mathbf{8 4 6}$ \\
\hline
\end{tabular}

\section{FLZ - Gesundheit: Normwerte BRD-West}

\begin{tabular}{|l|r|r|r|r|r|}
\hline Erhebung 1995 & Mean & \multicolumn{1}{c|}{ SD } & \multicolumn{1}{c|}{ Min } & Max & N \\
\hline Körperl. Leistungsfähigkeit & 8,23 & 7,05 & -12 & 20 & 1071 \\
\hline Entspannungsfähigkeit & 7,66 & 6,53 & -12 & 20 & 1067 \\
\hline Energie/Lebensfreude & 9,49 & 6,52 & -12 & 20 & 1068 \\
\hline Fortbewegungsfähigkeit & 8,98 & 6,91 & -12 & 20 & 1066 \\
\hline Seh-/Hörvermögen & 11,19 & 7,03 & -12 & 20 & 1069 \\
\hline Angstfreiheit & 8,35 & 6,66 & -12 & 20 & 1062 \\
\hline Beschwerde-/Schmerzfreiheit & 9,39 & 7,37 & -12 & 20 & 1070 \\
\hline Unabhängigkeit von Hilfe & 12,52 & 6,73 & -12 & 20 & 1068 \\
\hline FLZ-G Summenwert & $\mathbf{7 5 , 8 1}$ & $\mathbf{4 1 , 7 6}$ & $\mathbf{- 6 3}$ & $\mathbf{1 6 0}$ & $\mathbf{1 0 7 0}$ \\
\hline
\end{tabular}

\section{FLZ - Gesundheit: Normwerte BRD-Ost}

\begin{tabular}{|l|r|r|r|r|r|r|}
\hline Erhebung 1995 & \multicolumn{1}{c}{ Mean } & \multicolumn{1}{c}{ SD } & \multicolumn{1}{c}{ Min } & Max & N \\
\hline Körperl. Leistungsfähigkeit & 7,56 & 6,83 & -12 & 20 & 1150 \\
\hline Entspannungsfähigkeit & 6,40 & 6,29 & -12 & 20 & 1149 \\
\hline Energie/Lebensfreude & 7,86 & 6,45 & -12 & 20 & 1148 \\
\hline Fortbewegungsfähigkeit & 9,40 & 7,11 & -12 & 20 & 1146 \\
\hline Seh-/Hörvermögen & 10,44 & 6,98 & -12 & 20 & 1149 \\
\hline Angstfreiheit & 7,19 & 6,82 & -12 & 20 & 1145 \\
\hline Beschwerde-/Schmerzfreiheit & 8,00 & 7,37 & -12 & 20 & 1147 \\
\hline Unabhängigkeit von Hilfe & 12,21 & 6,67 & -12 & 20 & 1147 \\
\hline FLZ-G Summenwert & $\mathbf{6 9 , 0 6}$ & $\mathbf{4 0 , 2 7}$ & $\mathbf{- 6 8}$ & $\mathbf{1 6 0}$ & $\mathbf{1 1 4 9}$ \\
\hline
\end{tabular}

* ) Normwerte getrennt nach Alter und Geschlecht siehe Anhang B 


\section{ANHANG A: $\quad$ FLZ - Allgemeiner Teil • Normwerte BRD-West (1994)} getrennt nach Alter und Geschlecht

WZ1

$\begin{array}{lrrr} & \text { Mean } & \text { SD } & \text { N } \\ \text { 25 und jünger } & 10,93 & 7,17 & 185 \\ \text { 26 bis 35 } & 8,87 & 6,42 & 357 \\ \text { 36 bis 45 } & 8,04 & 6,20 & 278 \\ 46 \text { bis 55 } & 7,10 & 6,10 & 270 \\ 56 \text { bis 65 } & 8,26 & 6,49 & 303 \\ \text { älter als 65 } & 7,53 & 6,40 & 296 \\ \text { Total } & 8,33 & 6,51 & 1688\end{array}$

WZ2

25 und jünger
26 bis 35
36 bis 45
46 bis 55
56 bis 65
älter als 65
Total

WZ3

25 und jünger
26 bis 35
36 bis 45
46 bis 55
56 bis 65
älter als 65
Total

WZ4

\section{Freizeit / Hobbies}

$\begin{array}{rrr}\text { Mean } & \text { SD } & \text { N } \\ 7,90 & 6,86 & 186 \\ 6,81 & 5,84 & 356 \\ 5,97 & 6,03 & 278 \\ 6,19 & 6,08 & 271 \\ 7,55 & 6,90 & 304 \\ 6,26 & 6,28 & 293 \\ 6,73 & 6,33 & 1687\end{array}$

\section{Gesundheit}

$\begin{array}{rrr}\text { Mean } & \text { SD } & \text { N } \\ 9,39 & 7,08 & 186 \\ 9,39 & 6,79 & 358 \\ 9,66 & 7,33 & 279 \\ 7,87 & 7,38 & 271 \\ 8,04 & 8,17 & 304 \\ 5,99 & 7,83 & 296 \\ 8,35 & 7,55 & 1693\end{array}$

Männer

$\begin{array}{rrr}\text { Mean } & \text { SD } & \text { N } \\ 10,97 & 7,31 & 102 \\ 8,48 & 6,11 & 178 \\ 7,66 & 5,88 & 129 \\ 7,02 & 6,06 & 124 \\ 8,45 & 6,51 & 152 \\ 7,03 & 6,13 & 112 \\ 8,23 & 6,41 & 797\end{array}$

Männer

$\begin{array}{rrr}\text { Mean } & \text { SD } & \text { N } \\ 8,73 & 6,93 & 102 \\ 7,42 & 6,06 & 178 \\ 5,79 & 5,87 & 129 \\ 6,37 & 6,23 & 125 \\ 8,45 & 7,22 & 153 \\ 6,72 & 6,15 & 112 \\ 7,26 & 6,48 & 799\end{array}$

Frauen

$\begin{array}{rrr}\text { Mean } & \text { SD } & \text { N } \\ 6,91 & 6,69 & 85 \\ 6,20 & 5,56 & 177 \\ 6,12 & 6,18 & 149 \\ 6,05 & 5,98 & 146 \\ 6,64 & 6,46 & 151 \\ 5,97 & 6,36 & 180 \\ 6,26 & 6,15 & 889\end{array}$

Männer

$\begin{array}{rrr}\text { Mean } & \text { SD } & \text { N } \\ 9,46 & 7,01 & 102 \\ 8,99 & 6,77 & 178 \\ 8,94 & 7,36 & 130 \\ 6,74 & 7,12 & 125 \\ 8,23 & 8,43 & 153 \\ 6,39 & 7,49 & 112 \\ 8,18 & 7,45 & 800\end{array}$

Frauen

$\begin{array}{rrr}\text { Mean } & \text { SD } & \text { N } \\ 9,31 & 7,21 & 85 \\ 9,78 & 6,81 & 180 \\ 10,28 & 7,27 & 149 \\ 8,84 & 7,49 & 146 \\ 7,84 & 7,93 & 151 \\ 5,74 & 8,04 & 183 \\ 8,51 & 7,64 & 894\end{array}$

25 und jünger
26 bis 35
36 bis 45
46 bis 55
56 bis 65
älter als 65
Total

\section{Einkommen / finanzielle Sicherheit}

Männer

$\begin{array}{rrr}\text { Mean } & \text { SD } & \text { N } \\ 5,43 & 7,28 & 184 \\ 7,15 & 7,04 & 358 \\ 7,33 & 6,89 & 279 \\ 7,06 & 7,12 & 271 \\ 7,75 & 7,04 & 303 \\ 7,71 & 7,01 & 294 \\ 7,18 & 7,07 & 1690\end{array}$

$\begin{array}{rrr}\text { Mean } & \text { SD } & \text { N } \\ 4,49 & 7,44 & 102 \\ 7,49 & 7,38 & 178 \\ 6,59 & 7,28 & 130 \\ 6,74 & 7,14 & 125 \\ 7,67 & 7,15 & 153 \\ 8,93 & 7,03 & 112 \\ 7,08 & 7,32 & 800\end{array}$

Frauen

$\begin{array}{rrr}\text { Mean } & \text { SD } & \text { N } \\ 6,60 & 6,95 & 82 \\ 6,82 & 6,70 & 180 \\ 7,96 & 6,49 & 149 \\ 7,34 & 7,11 & 146 \\ 7,82 & 6,94 & 151 \\ 6,95 & 6,91 & 182 \\ 7,27 & 6,84 & 890\end{array}$


WZ5

25 und jünger
26 bis 35
36 bis 45
46 bis 55
56 bis 65
älter als 65
Total

WZ6

25 und jünger

26 bis 35

36 bis 45

46 bis 55

56 bis 65

älter als 65

Total

WZ7

25 und jünger
26 bis 35
36 bis 45
46 bis 55
56 bis 65
älter als 65
Total

\section{WZ8}

25 und jünger
26 bis 35
36 bis 45
46 bis 55
56 bis 65
älter als 65
Total

\section{Beruf / Arbeit}

$\begin{array}{rrr}\text { Mean } & \text { SD } & \text { N } \\ 6,03 & 7,24 & 183 \\ 6,64 & 6,78 & 356 \\ 6,88 & 7,05 & 276 \\ 6,21 & 7,25 & 271 \\ 6,20 & 7,58 & 289 \\ 4,12 & 6,07 & 266 \\ 6,06 & 7,04 & 1640\end{array}$

\section{Wohnsituation}

$\begin{array}{rrr}\text { Mean } & \text { SD } & \text { N } \\ 7,93 & 6,66 & 184 \\ 7,44 & 6,19 & 358 \\ 8,95 & 6,17 & 278 \\ 9,20 & 5,73 & 269 \\ 9,64 & 6,69 & 304 \\ 8,85 & 6,23 & 294 \\ 8,67 & 6,31 & 1687\end{array}$

Familie / Kinder

Männer

Mean SD N

$7,87 \quad 6,62 \quad 182$

$9,73 \quad 7,36 \quad 356$

$10,54 \quad 7,05 \quad 277$

$10,92 \quad 6,58 \quad 271$

$10,56 \quad 6,76 \quad 300$

$8,55 \quad 7,18 \quad 292$

$9,80 \quad 7,04 \quad 1678$
Männer

$\begin{array}{rrr}\text { Mean } & \text { SD } & \text { N } \\ 5,18 & 7,81 & 102 \\ 7,38 & 7,07 & 177 \\ 7,02 & 7,64 & 129 \\ 5,47 & 7,67 & 125 \\ 6,53 & 7,85 & 146 \\ 4,70 & 6,75 & 102 \\ 6,22 & 7,51 & 780\end{array}$

Frauen

Mean SD N

$7,10 \quad 6,33 \quad 81$

$5,91 \quad 6,41 \quad 179$

$6,76 \quad 6,51 \quad 147$

$6,84 \quad 6,83 \quad 146$

$5,86 \quad 7,31 \quad 143$

$3,76 \quad 5,59 \quad 164$

$5,91 \quad 6,59 \quad 860$

\begin{tabular}{rrrrrr}
\multicolumn{3}{c}{ Männer } & \multicolumn{3}{c}{ Frauen } \\
Mean & SD & N & Mean & SD & N \\
7,66 & 6,74 & 102 & 8,25 & 6,59 & 82 \\
7,44 & 5,98 & 178 & 7,45 & 6,41 & 180 \\
8,27 & 6,45 & 129 & 9,53 & 5,87 & 149 \\
9,52 & 5,72 & 125 & 8,93 & 5,74 & 144 \\
9,62 & 6,38 & 153 & 9,67 & 7,00 & 151 \\
9,40 & 6,37 & 112 & 8,51 & 6,13 & 182 \\
8,62 & 6,30 & 799 & 8,71 & 6,32 & 889
\end{tabular}

\section{Partnerschaft / Sexualität}

Männer

$\begin{array}{rrr}\text { Mean } & \text { SD } & \text { N } \\ 9,43 & 8,53 & 186 \\ 9,19 & 7,73 & 357 \\ 9,01 & 8,07 & 277 \\ 8,33 & 6,97 & 271 \\ 8,29 & 7,56 & 296 \\ 4,37 & 7,24 & 285 \\ 8,07 & 7,83 & 1672\end{array}$

\begin{tabular}{rrr}
\multicolumn{3}{c}{ Männer } \\
Mean & SD & N \\
9,09 & 8,36 & 101 \\
9,98 & 7,54 & 178 \\
9,53 & 8,12 & 128 \\
9,23 & 6,62 & 125 \\
8,76 & 7,25 & 147 \\
6,18 & 7,63 & 112 \\
8,91 & 7,64 & 792
\end{tabular}

$\begin{array}{rrr}\text { Mean } & \text { SD } & \text { N } \\ 6,72 & 6,30 & 101 \\ 9,25 & 6,96 & 177 \\ 9,82 & 7,60 & 128 \\ 10,32 & 6,51 & 125 \\ 10,33 & 6,65 & 150 \\ 9,47 & 7,23 & 111 \\ 9,43 & 6,97 & 792\end{array}$

Mean SD N

$9,30 \quad 6,76 \quad 81$

$10,21 \quad 7,72 \quad 179$

$11,16 \quad 6,50 \quad 149$

$11,44 \quad 6,62 \quad 146$

$10,79 \quad 6,89 \quad 150$

$7,98 \quad 7,11 \quad 180$

$10,13 \quad 7,08 \quad 885$

Frauen

$\begin{array}{rrr}\text { Mean } & \text { SD } & \text { N } \\ 9,84 & 8,76 & 85 \\ 8,41 & 7,85 & 179 \\ 8,56 & 8,03 & 149 \\ 7,56 & 7,19 & 146 \\ 7,82 & 7,85 & 148 \\ 3,20 & 6,74 & 173 \\ 7,31 & 7,93 & 880\end{array}$


WZS

25 und jünger
26 bis 35
36 bis 45
46 bis 55
56 bis 65
älter als 65
Total

\section{FLZ-A Summenwert}

\begin{tabular}{rrrrrrrrr} 
& \multicolumn{4}{c}{ Männer } & \multicolumn{3}{c}{ Frauen } \\
Mean & SD & N & Mean & SD & N & Mean & SD & N \\
64,70 & 36,24 & 184 & 62,37 & 37,29 & 102 & 67,57 & 34,91 & 82 \\
65,29 & 35,65 & 358 & 66,44 & 33,84 & 178 & 64,15 & 37,42 & 180 \\
66,26 & 39,14 & 278 & 63,41 & 40,47 & 129 & 68,71 & 37,92 & 149 \\
62,91 & 35,98 & 271 & 61,37 & 34,67 & 125 & 64,22 & 37,14 & 146 \\
66,48 & 40,50 & 304 & 68,37 & 41,88 & 153 & 64,56 & 39,09 & 151 \\
53,75 & 37,49 & 293 & 59,05 & 38,80 & 112 & 50,44 & 36,37 & 180 \\
63,21 & 37,79 & 1688 & 63,97 & 37,85 & 799 & 62,53 & 37,75 & 889
\end{tabular}

\section{ANHANG B: $\quad$ FLZ - Gesundheit • Normwerte BRD-West (1995) getrennt nach Alter und Geschlecht}


WZ1

25 und jünger
26 bis 35
36 bis 45
46 bis 55
56 bis 65
älter als 65
Total

WZ2

$\begin{array}{lrrr} & \text { Mean } & \text { SD } & \text { N } \\ \text { 25 und jünger } & 9,39 & 7,33 & 138 \\ \text { 26 bis 35 } & 8,34 & 7,09 & 230 \\ \text { 36 bis 45 } & 8,04 & 6,42 & 167 \\ 46 \text { bis 55 } & 6,14 & 5,81 & 181 \\ 56 \text { bis 65 } & 6,93 & 6,28 & 176 \\ \text { älter als 65 } & 7,36 & 5,71 & 176 \\ \text { Total } & 7,66 & 6,53 & 1067\end{array}$

WZ3

25 und jünger
26 bis 35
36 bis 45
46 bis 55
56 bis 65
älter als 65
Total

\section{WZ4}

25 und jünger
26 bis 35
36 bis 45
46 bis 55
56 bis 65
älter als 65
Total

Körperl. Leistungsfähigkeit

\section{Energie / Lebensfreude}

$\begin{array}{rrr}\text { Mean } & \text { SD } & \text { N } \\ 11,96 & 6,89 & 136 \\ 11,05 & 7,05 & 229 \\ 9,40 & 5,69 & 167 \\ 8,63 & 5,73 & 182 \\ 8,54 & 6,04 & 178 \\ 7,47 & 6,49 & 176 \\ 9,49 & 6,52 & 1068\end{array}$

\section{Fortbewegungsfähigkeit}

\begin{tabular}{rrrrrrrrr} 
& & \multicolumn{3}{c}{ Männer } & \multicolumn{3}{c}{ Frauen } \\
Mean & SD & N & Mean & SD & N & Mean & SD & N \\
11,40 & 6,22 & 138 & 12,30 & 5,92 & 79 & 10,19 & 6,46 & 59 \\
10,33 & 6,83 & 230 & 10,79 & 7,27 & 113 & 9,88 & 6,38 & 117 \\
9,34 & 6,34 & 167 & 9,11 & 6,54 & 81 & 9,55 & 6,17 & 86 \\
7,76 & 5,89 & 183 & 8,70 & 5,73 & 82 & 7,00 & 5,93 & 101 \\
6,40 & 7,20 & 178 & 5,30 & 8,09 & 87 & 7,44 & 6,10 & 91 \\
4,29 & 7,21 & 176 & 4,34 & 7,78 & 63 & 4,26 & 6,91 & 113 \\
8,23 & 7,05 & 1071 & 8,67 & 7,43 & 505 & 7,84 & 6,67 & 566
\end{tabular}

\begin{tabular}{rrrrrr}
\multicolumn{3}{c}{ Männer } & \multicolumn{3}{c}{ Frauen } \\
Mean & SD & N & Mean & SD & N \\
9,38 & 6,92 & 79 & 9,40 & 7,91 & 59 \\
9,43 & 7,48 & 113 & 7,29 & 6,55 & 117 \\
7,77 & 6,53 & 81 & 8,30 & 6,34 & 86 \\
6,53 & 5,95 & 82 & 5,83 & 5,71 & 99 \\
7,32 & 6,29 & 87 & 6,55 & 6,29 & 89 \\
7,74 & 5,49 & 63 & 7,14 & 5,84 & 113 \\
8,11 & 6,63 & 505 & 7,26 & 6,41 & 562
\end{tabular}

$\begin{array}{rrr} & \text { Männer } \\ \text { Mean } & \text { SD } & \text { N } \\ 11,76 & 7,05 & 79 \\ 11,73 & 7,00 & 112 \\ 8,60 & 5,24 & 81 \\ 8,82 & 5,54 & 82 \\ 8,30 & 6,19 & 87 \\ 7,45 & 7,02 & 63 \\ 9,63 & 6,59 & 504\end{array}$

$\begin{array}{rrr}\text { Mean } & \text { SD } & \text { N } \\ 12,22 & 6,72 & 57 \\ 10,39 & 7,07 & 117 \\ 10,15 & 6,01 & 86 \\ 8,47 & 5,91 & 101 \\ 8,76 & 5,91 & 91 \\ 7,48 & 6,20 & 113 \\ 9,35 & 6,45 & 564\end{array}$

$\begin{array}{rrr}\text { Mean } & \text { SD } & \mathrm{N} \\ 9,48 & 6,92 & 138 \\ 11,51 & 6,62 & 229 \\ 10,42 & 6,90 & 167 \\ 9,05 & 5,79 & 182 \\ 7,92 & 6,03 & 174 \\ 4,92 & 7,24 & 176 \\ 8,98 & 6,91 & 1066\end{array}$

Männer

\begin{tabular}{rrr}
\multicolumn{3}{c}{ Männer } \\
Mean & SD & $\mathrm{N}$ \\
9,52 & 7,00 & 79 \\
12,59 & 6,76 & 112 \\
9,84 & 6,49 & 81 \\
9,71 & 5,89 & 82 \\
8,56 & 6,10 & 83 \\
6,19 & 7,56 & 63 \\
9,71 & 6,86 & 500
\end{tabular}

Frauen

$\begin{array}{rrr}\text { Mean } & \text { SD } & \text { N } \\ 9,43 & 6,86 & 59 \\ 10,46 & 6,35 & 117 \\ 10,96 & 7,25 & 86 \\ 8,52 & 5,69 & 101 \\ 7,34 & 5,94 & 91 \\ 4,21 & 6,98 & 113 \\ 8,34 & 6,90 & 566\end{array}$


WZ5

25 und jünger
26 bis 35
36 bis 45
46 bis 55
56 bis 65
älter als 65
Total

WZ6

25 und jünger
26 bis 35
36 bis 45
46 bis 55
56 bis 65
älter als 65
Total

WZ7

25 und jünger
26 bis 35
36 bis 45
46 bis 55
56 bis 65
älter als 65
Total

\section{WZ8}

$\begin{array}{lrrr} & \text { Mean } & \text { SD } & \text { N } \\ \text { 25 und jünger } & 13,97 & 6,51 & 138 \\ \text { 26 bis 35 } & 13,99 & 6,64 & 229 \\ \text { 36 bis 45 } & 13,93 & 5,83 & 167 \\ \text { 46 bis 55 } & 12,30 & 6,17 & 181 \\ 56 \text { bis 65 } & 11,32 & 6,41 & 178 \\ \text { älter als 65 } & 9,57 & 7,49 & 176 \\ \text { Total } & 12,52 & 6,73 & 1068\end{array}$

Seh- / Hörvermögen

\section{Angstfreiheit}

\section{Unabhängigkeit von Hilfe}

\begin{tabular}{rrrrrrrrr} 
& & \multicolumn{3}{c}{ Männer } & \multicolumn{3}{c}{ Frauen } \\
Mean & SD & N & Mean & SD & N & Mean & SD & N \\
15,05 & 5,59 & 138 & 14,83 & 5,85 & 79 & 15,36 & 5,25 & 59 \\
13,39 & 6,71 & 228 & 14,24 & 7,19 & 112 & 12,57 & 6,12 & 116 \\
12,44 & 6,51 & 167 & 11,87 & 6,49 & 81 & 12,97 & 6,53 & 86 \\
10,80 & 6,67 & 182 & 11,19 & 5,90 & 82 & 10,49 & 7,26 & 101 \\
9,15 & 5,70 & 178 & 9,46 & 5,96 & 87 & 8,86 & 5,45 & 91 \\
6,60 & 7,39 & 176 & 6,59 & 7,68 & 63 & 6,60 & 7,26 & 113 \\
11,19 & 7,03 & 1069 & 11,68 & 7,04 & 504 & 10,76 & 7,00 & 565
\end{tabular}

\begin{tabular}{rrrrrrrrr} 
& & \multicolumn{3}{c}{ Männer } & \multicolumn{3}{c}{ Frauen } \\
Mean & SD & N & Mean & SD & N & Mean & SD & N \\
9,99 & 6,73 & 135 & 9,88 & 6,98 & 79 & 10,14 & 6,43 & 56 \\
9,45 & 6,98 & 229 & 10,14 & 7,10 & 112 & 8,78 & 6,83 & 117 \\
9,32 & 6,45 & 163 & 8,29 & 5,72 & 81 & 10,33 & 6,99 & 82 \\
7,61 & 5,82 & 182 & 7,95 & 5,47 & 82 & 7,34 & 6,11 & 101 \\
6,94 & 6,66 & 178 & 6,97 & 8,26 & 87 & 6,91 & 4,71 & 91 \\
6,95 & 6,59 & 176 & 8,23 & 5,95 & 63 & 6,24 & 6,84 & 113 \\
8,35 & 6,66 & 1062 & 8,66 & 6,79 & 504 & 8,06 & 6,54 & 559
\end{tabular}

\section{Beschwerde- / Schmerzfreiheit}

Männer

$\begin{array}{rrr}\text { Mean } & \text { SD } & \text { N } \\ 12,69 & 6,63 & 138 \\ 11,93 & 7,08 & 229 \\ 11,04 & 6,64 & 167 \\ 7,75 & 6,49 & 182 \\ 6,85 & 7,26 & 178 \\ 6,18 & 7,34 & 176 \\ 9,39 & 7,37 & 1070\end{array}$

$\begin{array}{rrr}\text { Mean } & \text { SD } & \text { N } \\ 11,96 & 7,05 & 79 \\ 12,31 & 7,43 & 112 \\ 10,25 & 7,11 & 81 \\ 8,35 & 6,61 & 82 \\ 5,61 & 8,14 & 87 \\ 6,65 & 7,10 & 63 \\ 9,42 & 7,69 & 504\end{array}$

Frauen

$\begin{array}{rrr}\text { Mean } & \text { SD } & \text { N } \\ 13,68 & 5,95 & 59 \\ 11,56 & 6,74 & 117 \\ 11,78 & 6,11 & 86 \\ 7,26 & 6,39 & 101 \\ 8,03 & 6,13 & 91 \\ 5,92 & 7,50 & 113 \\ 9,36 & 7,08 & 566\end{array}$

Männer

$\begin{array}{rrr}\text { Mean } & \text { SD } & \text { N } \\ 13,07 & 6,45 & 79 \\ 14,22 & 7,06 & 112 \\ 13,06 & 5,67 & 81 \\ 13,00 & 5,87 & 82 \\ 10,70 & 6,82 & 87 \\ 9,72 & 7,02 & 63 \\ 12,49 & 6,67 & 504\end{array}$

Frauen

$\begin{array}{rrr}\text { Mean } & \text { SD } & \text { N } \\ 15,19 & 6,45 & 59 \\ 13,77 & 6,24 & 117 \\ 14,76 & 5,88 & 86 \\ 11,73 & 6,38 & 100 \\ 11,90 & 5,97 & 91 \\ 9,48 & 7,77 & 113 \\ 12,55 & 6,79 & 565\end{array}$


WZS

\begin{tabular}{lrrrrrrrrr} 
& \multicolumn{3}{c}{} & \multicolumn{4}{c}{ Männer } & \multicolumn{3}{c}{ Frauen } \\
& Mean & SD & $\mathrm{N}$ & Mean & SD & N & Mean & SD & N \\
25 und jünger & 93,99 & 38,74 & 138 & 92,70 & 37,93 & 79 & 95,74 & 40,07 & 59 \\
26 bis 35 & 90,03 & 42,56 & 229 & 95,60 & 45,67 & 112 & 84,67 & 38,78 & 117 \\
36 bis 45 & 83,83 & 39,22 & 167 & 78,78 & 37,92 & 81 & 88,57 & 40,04 & 86 \\
46 bis 55 & 70,01 & 33,70 & 182 & 74,24 & 34,16 & 82 & 66,59 & 33,09 & 101 \\
56 bis 65 & 64,07 & 36,86 & 178 & 62,27 & 41,16 & 87 & 65,78 & 32,39 & 91 \\
älter als 65 & 53,35 & 42,95 & 176 & 56,92 & 42,28 & 63 & 51,34 & 43,39 & 113 \\
Total & 75,81 & 41,76 & 1070 & 78,40 & 42,60 & 504 & 73,50 & 40,91 & 566
\end{tabular}

\section{Kontaktadresse:}

Dr. Gerhard Henrich, Dr. Peter Herschbach Institut und Poliklinik für Psychosomatische Medizin, Psychotherapie und Medizinische Psychologie der Technischen Universität München

Langerstraße 3, 81675 München

Telefon: $+49-89-41404318$

Fax: $\quad+49-89-41404916$

E-mail: G.Henrich@lrz.tu-muenchen.de 


\section{Fragen zur Lebenszufriedenheit (FLZ)}

Bei den folgenden Fragen geht es darum, wie zufrieden Sie mit Ihrem Leben und miteinzelnen Aspekten ihres Lebens sind. Außerdem sollen Sie angeben, wie wichtig einzelne Lebensbereiche (z.B. Beruf oder Freizeit) für Ihre Zufriedenheit und Ihr Wohlbefinden sind.

Bitte beantworten Sie alle Fragen, auch diejenigen, die scheinbar nicht auf Sie zutreffen:

Wenn Sie z.B. keinen Partner haben, können Sie bei der Frage nach der "Partnerschaft" trotzdem angeben, wie wichtig Ihnen das wäre und wie zufrieden Sie mit der derzeitigen Situation (ohne Partner ) sind.

Lassen Sie sich nicht davon beeinflussen, ob Sie sich im Augenblick gut oder schlecht fühlen, sondern versuchen Sie, bei Ihrer Beurteilung die letzten vier Wochen zu berücksichtigen.

Bitte kreuzen Sie zunächst an, wie wichtig jeder einzelne Lebensbereich für Ihre Zufriedenheit insgesamt ist. Bevor Sie beginnen, schauen Sie bitte erst alle Bereiche an.

Wie wichtig ist ( sind ) für Sie......

1. Freunde / Bekannte

2. Freizeitgestaltung / Hobbies

3 Gesundheit

4. Einkommen / finanzielle Sicherheit

5. Beruf / Arbeit

6. Wohnsituation

7. Familienleben / Kinder

8. Partnerschaft / Sexualität

$$
\text { nicht etwas }
$$$$
\text { wichtig wichtig }
$$

$$
\begin{gathered}
\text { ziemlich sehr } \\
\text { wichtig wichtig }
\end{gathered}
$$

extrem wichtig

Bitte kreuzen Sie nun an, wie zufrieden Sie in den einzelnen Lebensbereichen sind.

Wie zufrieden sind Sie mit.......

1. Freunde / Bekannte

2. Freizeitgestaltung / Hobbies

3 Gesundheit

4. Einkommen / finanzielle Sicherheit

5. Beruf / Arbeit

6. Wohnsituation

7. Familienleben / Kinder

8. Partnerschaft / Sexualität $\begin{array}{llc}\text { un- } & \text { eher un- } & \text { eher } \\ \text { zufrieden } & \text { zufrieden } & \text { zufrieden }\end{array}$

ziemlich zufrieden zufrieden zufrieden zufrieden zufrieden

Wie zufrieden sind Sie mit Ihrem Leben insgesamt, wenn Sie alle Aspekte zusammennehmen? 


\section{FLZ Türkische Version | Hayat Memnuniyeti Anketi}

Sunduğumuz bu sorularn amacı, hayatınızdan ve hayatınızın bazi bölümlerinden ne kadar memnun olup olmadığnızı öğrenmektir.

Bundan hariç öğrenmek istediğimiz, hayatınızın özel bölümleri (iş veya boş vakit gibi) bu memnuniyet için ne kadar önemlidir.

Bütün bu soruları, sizinle ilgili olmasa bile, lütfen cevaplayınız: misal olarak, bir eşiniz olmasada, eşlik-hayatı sorusu hakkında, sizin için bu ne kadar önemli olduğunu ve şu anki durumunuzla (yani eşsiz) ne kadar memnun olduğunzu dile getirebilirsiniz.

Şu anki durumunuz, (belki şu an kendinizi kötü, belkide şu iyi hissediyorsunuz) sizi etkilemesin. Vereceğiniz cevabınızda son dört haftanızı lütfen göz önünüze alınız.

Lütfen öncelikle, her bir hayat bölümünün sizin genel memnuniyetiniz açısından ne kadar önemli olup olmadığını işaretleyiniz. Başlamadan evvel bütün bölümleri bir gözden geçirin lütfen.

Sizin için ... ne kadar önemlidir ?

\begin{tabular}{|l|c|c|c|c|c|}
\hline & $\begin{array}{c}\text { Önemli } \\
\text { Değil }\end{array}$ & $\begin{array}{c}\text { Az } \\
\text { onemli }\end{array}$ & $\begin{array}{c}\text { Oldukca } \\
\text { önemli }\end{array}$ & $\begin{array}{c}\text { Çok } \\
\text { onemli }\end{array}$ & $\begin{array}{c}\text { Aşırı } \\
\text { derecede } \\
\text { önemli }\end{array}$ \\
\hline$-\quad$ Arkadaşlar / Tanıdıklar & $\square$ & $\square$ & $\square$ & $\square$ & $\square$ \\
\hline$-\quad$ Boş zaman değerlendirimi / Hobiler & $\square$ & $\square$ & $\square$ & $\square$ & $\square$ \\
\hline$-\quad$ Sağlık & $\square$ & $\square$ & $\square$ & $\square$ & $\square$ \\
\hline$-\quad$ Gelir / Maddi emniyet & $\square$ & $\square$ & $\square$ & $\square$ & $\square$ \\
\hline$-\quad$ Meslek / İş & $\square$ & $\square$ & $\square$ & $\square$ & $\square$ \\
\hline$-\quad$ Ikamet durumu & $\square$ & $\square$ & $\square$ & $\square$ & $\square$ \\
\hline$-\quad$ Aile hayatı / Çocuklar & $\square$ & $\square$ & $\square$ & $\square$ & $\square$ \\
\hline$-\quad$ Eşlik hayatı / Cinsellik & $\square$ & $\square$ & $\square$ & $\square$ & $\square$ \\
\hline
\end{tabular}

Şimdide sunduğumuz her bir hayat bölümü açısında şu anda ne kadar memnun olduğunuzu lütfen işaretleyiniz:

... konusunda ne kadar memnunsunuz:

\begin{tabular}{|l|c|c|c|c|c|}
\hline & $\begin{array}{c}\text { Memnun } \\
\text { Değil }\end{array}$ & $\begin{array}{c}\text { Az } \\
\text { memnun }\end{array}$ & $\begin{array}{c}\text { Oldukca } \\
\text { memnun }\end{array}$ & $\begin{array}{c}\text { Çok } \\
\text { memnun }\end{array}$ & $\begin{array}{c}\text { Aşırı } \\
\text { derecede } \\
\text { memnun }\end{array}$ \\
\hline$\bullet \quad$ Arkadaşlar / Tanıdıklar & $\square$ & $\square$ & $\square$ & $\square$ & $\square$ \\
\hline$\bullet \quad$ Boş zaman değerlendirimi / Hobiler & $\square$ & $\square$ & $\square$ & $\square$ \\
\hline$\bullet \quad$ Sağlık & $\square$ & $\square$ & $\square$ & $\square$ \\
\hline$\bullet \quad$ Gelir / Maddi emniyet & $\square$ & $\square$ & $\square$ & $\square$ \\
\hline$\bullet \quad$ Meslek / İş & $\square$ & $\square$ & $\square$ & $\square$ & $\square$ \\
\hline$\bullet \quad$ İkamet durumu & $\square$ & $\square$ & $\square$ & $\square$ \\
\hline$\bullet \quad$ Aile hayatı / Çocuklar & $\square$ & $\square$ & $\square$ \\
\hline$\bullet \quad$ Eşlik hayatı / Cinsellik & $\square$ & $\square$ & $\square$ & $\square$ \\
\hline
\end{tabular}


Fragebogenerhebung - Häufigkeiten

Statistiken

\begin{tabular}{|c|c|c|c|c|c|c|c|}
\hline & 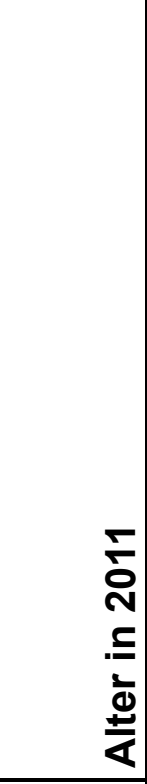 & 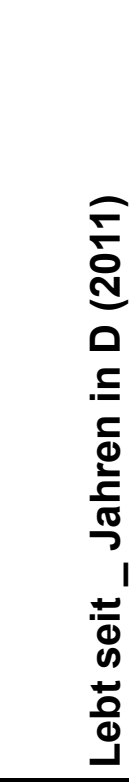 & 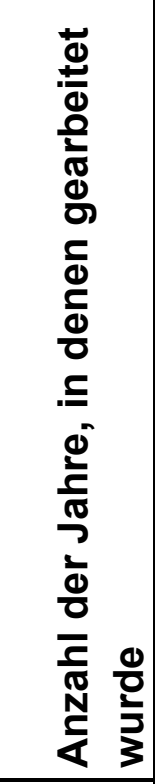 & 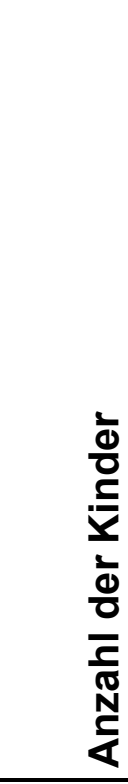 & 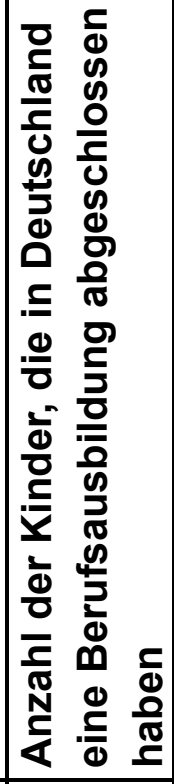 & 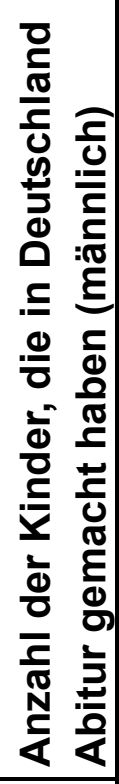 & 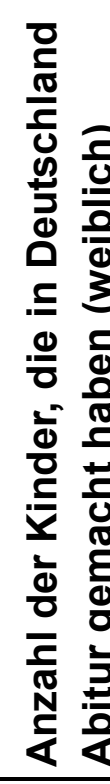 \\
\hline N Gültig & 67 & 63 & 54 & 65 & 59 & 55 & 54 \\
\hline Fehlend & 0 & 4 & 13 & 2 & 8 & 12 & 13 \\
\hline Mittelwert & 55,91 & 34,02 & 21,87 & 2,69 & ,58 & ,24 & 17 \\
\hline Median & 55,00 & 38,00 & 22,00 & 2,00 & ,00 & ,00 & ,00 \\
\hline Standardabweichung & 11,274 & 9,713 & 11,244 & 1,310 & 724 & ,744 & ,423 \\
\hline Minimum & 38 & 7 & 0 & 1 & 0 & 0 & 0 \\
\hline Maximum & 78 & 48 & 44 & 9 & 2 & 3 & 2 \\
\hline Summe & 3746 & 2143 & 1181 & 175 & 34 & 13 & 9 \\
\hline
\end{tabular}


Fragebogenerhebung - Häufigkeitstabellen

\begin{tabular}{|rl|r|r|r|r|}
\hline & Häufigkeit & Prozent & Gültige Prozente & Kumulierte Prozente \\
\hline \multirow{2}{*}{ Gültig männlich } & 35 & 52,2 & 52,2 & 52,2 \\
& weiblich & 32 & 47,8 & 47,8 & 100,0 \\
& 67 & 100,0 & 100,0 & \\
\hline
\end{tabular}

Staatsangehörigkeit

\begin{tabular}{|c|c|c|c|c|c|}
\hline & & Häufigkeit & Prozent & Gültige Prozente & Kumulierte Prozente \\
\hline \multirow[t]{4}{*}{ Gültig } & türkisch & 46 & 68,7 & 68,7 & 68,7 \\
\hline & deutsch & 16 & 23,9 & 23,9 & 92,5 \\
\hline & andere & 5 & 7,5 & 7,5 & 100,0 \\
\hline & Gesamt & 67 & 100,0 & 100,0 & \\
\hline
\end{tabular}

Familienstand

\begin{tabular}{|ll|r|r|r|r|}
\hline & Häufigkeit & Prozent & Gültige Prozente & Kumulierte Prozente \\
\hline \multirow{2}{*}{ Gültig } & $\mathbf{5 7}$ & $\mathbf{8 5 , 1}$ & 85,1 & 85,1 \\
& verheiratet & $\mathbf{3}$ & $\mathbf{4 , 5}$ & 4,5 & 89,6 \\
& ledig & $\mathbf{5}$ & $\mathbf{7 , 5}$ & 7,5 & 97,0 \\
verwitwet & $\mathbf{5}$ & 1,5 & 1,5 & 98,5 \\
geschieden & $\mathbf{1}$ & 1,5 & 1,5 & 100,0 \\
getrennt & 67 & 100,0 & 100,0 & \\
Gesamt & & & & \\
\hline
\end{tabular}




\begin{tabular}{|c|c|c|c|c|c|}
\hline \multicolumn{6}{|c|}{$\begin{array}{l}\text { Lebt seit _ Jahren in Deutschland } \\
\text { (Referenz: 2011) }\end{array}$} \\
\hline & & Häufigkeit & Prozent & $\begin{array}{c}\text { Gültige } \\
\text { Prozente }\end{array}$ & $\begin{array}{c}\text { Kumulierte } \\
\text { Prozente }\end{array}$ \\
\hline \multirow[t]{22}{*}{ Gültig } & 7 & 1 & 1,5 & 1,6 & 1,6 \\
\hline & 9 & 2 & 3,0 & 3,2 & 4,8 \\
\hline & 20 & 3 & 4,5 & 4,8 & 9,5 \\
\hline & 21 & 5 & 7,5 & 7,9 & 17,5 \\
\hline & 22 & 1 & 1,5 & 1,6 & 19,0 \\
\hline & 23 & 1 & 1,5 & 1,6 & 20,6 \\
\hline & 25 & 1 & 1,5 & 1,6 & 22,2 \\
\hline & 28 & 1 & 1,5 & 1,6 & 23,8 \\
\hline & 30 & 1 & 1,5 & 1,6 & 25,4 \\
\hline & 31 & 2 & 3,0 & 3,2 & 28,6 \\
\hline & 32 & 5 & 7,5 & 7,9 & 36,5 \\
\hline & 34 & 5 & 7,5 & 7,9 & 44,4 \\
\hline & 36 & 1 & 1,5 & 1,6 & 46,0 \\
\hline & 38 & 7 & 10,4 & 11,1 & 57,1 \\
\hline & 39 & 7 & 10,4 & 11,1 & 68,3 \\
\hline & 40 & 1 & 1,5 & 1,6 & 69,8 \\
\hline & 41 & 8 & 11,9 & 12,7 & 82,5 \\
\hline & 42 & 2 & 3,0 & 3,2 & 85,7 \\
\hline & 43 & 4 & 6,0 & 6,3 & 92,1 \\
\hline & 45 & 2 & 3,0 & 3,2 & 95,2 \\
\hline & 48 & 3 & 4,5 & 4,8 & 100,0 \\
\hline & Gesamt & 63 & 94,0 & 100,0 & \\
\hline Fehlend & System & 4 & 6,0 & & \\
\hline Gesamt & & 67 & 100,0 & & \\
\hline
\end{tabular}


Beruf

(eigene Angaben meist in türk. Sprache)

\begin{tabular}{|c|c|c|c|c|c|c|}
\hline & & (Übersetzung, I.Ö.) & Häufigkeit & Prozent & $\begin{array}{c}\text { Gültige } \\
\text { Prozente }\end{array}$ & $\begin{array}{c}\text { Kumulierte } \\
\text { Prozente }\end{array}$ \\
\hline \multirow[t]{24}{*}{ Gültig } & & (keine Angabe) & 13 & 19,4 & 19,4 & 19,4 \\
\hline & Asci & Koch/Köchin & 1 & 1,5 & 1,5 & 20,9 \\
\hline & Doktor yardimcisi & Arzthelfer(-in) & 1 & 1,5 & 1,5 & 22,4 \\
\hline & emekli & Rentnerln & 4 & 6,0 & 6,0 & 28,4 \\
\hline & ev hanimi & Hausfrau & 2 & 3,0 & 3,0 & 31,3 \\
\hline & Fabrikatör & Fabrikateur & 1 & 1,5 & 1,5 & 32,8 \\
\hline & halici & Teppichhändler & 1 & 1,5 & 1,5 & 34,3 \\
\hline & insaat & Ваu & 4 & 6,0 & 6,0 & 40,3 \\
\hline & isci & Arbeiter(-in) & 19 & 28,4 & 28,4 & 68,7 \\
\hline & kaynakci & Schweisserln & 2 & 3,0 & 3,0 & 71,6 \\
\hline & kuru pastaci & Keksbäckerln & 1 & 1,5 & 1,5 & 73,1 \\
\hline & makine & Maschineneinstel & 1 & 1,5 & 1,5 & 74,6 \\
\hline & tesviyecisi & lerln & & & & \\
\hline & memur & Beamter(-in) & 1 & 1,5 & 1,5 & 76,1 \\
\hline & Metal Arbeiter & Metal Arbeiter & 1 & 1,5 & 1,5 & 77,6 \\
\hline & montör & Monteurln & 1 & 1,5 & 1,5 & 79,1 \\
\hline & mühendis & Ingeneurln & 1 & 1,5 & 1,5 & 80,6 \\
\hline & serbest & Frei & 2 & 3,0 & 3,0 & 83,6 \\
\hline & temizlikci & Reinigerln & 4 & 6,0 & 6,0 & 89,6 \\
\hline & terzi & Schneiderln & 3 & 4,5 & 4,5 & 94,0 \\
\hline & Töpfer & Töpfer & 1 & 1,5 & 1,5 & 95,5 \\
\hline & Tornacilik & DreherIn & 1 & 1,5 & 1,5 & 97,0 \\
\hline & yok & kein & 2 & 3,0 & 3,0 & 100,0 \\
\hline & Gesamt & & 67 & 100,0 & 100,0 & \\
\hline
\end{tabular}




\begin{tabular}{|ll|r|r|r|r|}
\hline & & Häufigkeit & Prozent & Gültige Prozente & Kumulierte Prozente \\
\hline Gültig & 1 & 7 & 10,4 & 10,8 & 10,8 \\
& 2 & $\mathbf{2 6}$ & $\mathbf{3 8 , 8}$ & 40,0 & 50,8 \\
& 21 & $\mathbf{3 1 , 3}$ & 32,3 & 83,1 \\
& 4 & $\mathbf{2 1}$ & $\mathbf{1 1 , 9}$ & 12,3 & 95,4 \\
& 6 & 2 & 3,0 & 3,1 & 98,5 \\
& 9 & 1 & 1,5 & 1,5 & 100,0 \\
& Gesamt & 65 & 97,0 & 100,0 & \\
Fehlend & System & 2 & 3,0 & & \\
Gesamt & & 67 & 100,0 & & \\
\hline
\end{tabular}

Anzahl der Kinder, die in Deutschland eine Berufsausbildung abgeschlossen haben

\begin{tabular}{|ll|r|r|r|r|}
\hline & & Häufigkeit & Prozent & Gültige Prozente & Kumulierte Prozente \\
\hline \multirow{2}{*}{ Gültig } & 0 & 33 & 49,3 & 55,9 & 55,9 \\
& 1 & 18 & 26,9 & 30,5 & 86,4 \\
& 2 & 8 & 11,9 & 13,6 & 100,0 \\
& Gesamt & 59 & 88,1 & 100,0 & \\
Fehlend & System & 8 & 11,9 & & \\
Gesamt & & 67 & 100,0 & & \\
\hline
\end{tabular}

Anzahl der Kinder, die in Deutschland Abitur gemacht haben (männlich)

\begin{tabular}{|ll|r|r|r|r|}
\hline & & Häufigkeit & Prozent & Gültige Prozente & Kumulierte Prozente \\
\hline \multirow{6}{*}{ Gültig } & 0 & $\mathbf{4 9}$ & $\mathbf{7 3 , 1}$ & 89,1 & 89,1 \\
& 1 & $\mathbf{2}$ & $\mathbf{3 , 0}$ & $\mathbf{3 , 6}$ & 92,7 \\
& 2 & $\mathbf{1}$ & $\mathbf{1 , 5}$ & $\mathbf{1 , 8}$ & 94,5 \\
& 3 & $\mathbf{3}$ & $\mathbf{4 , 5}$ & $\mathbf{5 , 5}$ & 100,0 \\
& Gesamt & 55 & 82,1 & 100,0 & \\
Fehlend & System & 12 & 17,9 & & \\
Gesamt & & 67 & 100,0 & & \\
\hline
\end{tabular}

Anzahl der Kinder, die in Deutschland Abitur gemacht haben (weiblich)

\begin{tabular}{|ll|r|r|r|r|}
\hline & & Häufigkeit & Prozent & Gültige Prozente & Kumulierte Prozente \\
\hline \multirow{2}{*}{ Gültig } & 0 & $\mathbf{4 6}$ & $\mathbf{6 8 , 7}$ & 85,2 & 85,2 \\
& 1 & $\mathbf{7}$ & $\mathbf{1 0 , 4}$ & $\mathbf{1 3 , 0}$ & 98,1 \\
& 2 & $\mathbf{1}$ & $\mathbf{1 , 5}$ & $\mathbf{1 , 9}$ & 100,0 \\
& Gesamt & 54 & 80,6 & 100,0 & \\
Fehlend & System & 13 & 19,4 & & \\
Gesamt & & 67 & 100,0 & & \\
\hline
\end{tabular}


Anhang Tab. 3.3 Systematik der Erklärungsansätze der Akkulturationsforschung (Zick, 2010: 126ff)

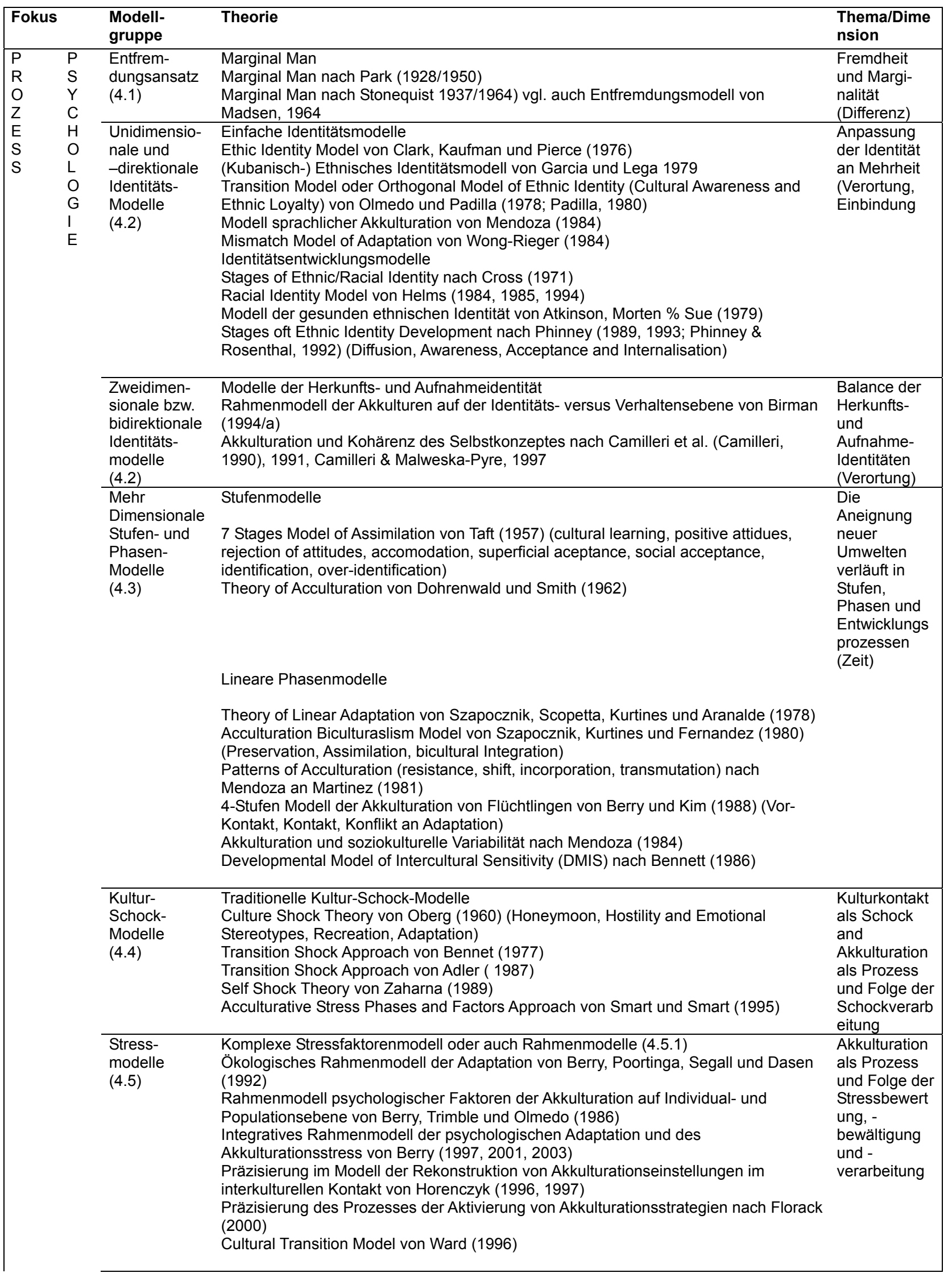


Komplexe (moderne) Schock- und Stressmodelle (4.5.2.)

Verhaltensoptionen und Coping (Adherence, Substitution, Selective Addition, Hybridization, Innovation) nach Seelye (1996), Seelye und Wasilewski (1996) Stress Process Model of Acculturation von Flakserud und Uman (1996)

Stressmodell der soziokulturellen Adaptation von Berry (1997)

Model Migration Contingencies and Acculturative Stress von Liebkind (1996a, in

Anlehnung an Berry)

Integrated Model of Acculturation and Intercultural Behavior Process von Landis and Bhawuk (2004, in Anlehnung an Berry)

$\mathrm{A}(\mathrm{ffect}) \mathrm{B}$ (ehavior)C(ognition)-Modell der Akkulturation von Ward, Bochner udnFurnahm (2001)

Bewältigung von Angst, Unsicherheit, und Bedrohung (4.5.3)

Uncertainty Reduction Based Theory of Intercultural Adaptation (AUM) von Gudykunst und Hammer (1988; Gudykunst, 1995, 1998)

Akkulturation und Kontrolle- Entscheidungs- und Aufgabenmodelle (4.5.4)

Internales Modell der Auswanderungsentscheidung und Adaptation von Lüthke (1989;

Lüthke \& Cropley, 1989)

Ökologisches Modell der Entwicklungsaufgaben und Kontrolle (Akkulturation als

Lernprozess) von Ruddat (1994; nach Bronfenbrenner, 1986)

Lern-Modelle
(4.6)

Lernprinzipien und -strukturen

Acculturation as Learning of Attitudes and Behavior Approach von Marin (1992)

Ansatz zur Interkulturellen Adaptation und Partizipation nach Anderson (1994)

Culture Learning Approach von Bochner (1986) und Ward (1997)

Adaptation

als

Lernprozess

und Lernziel

Hypothesen zum Lern- und Adaptationsverlauf (4.4)

(Differenz)

U-Curve Hypothesis von Lysgaard (1995; Church, 1982)

W-Curve Hypothesis (Brein \& David, 1971; Gullahorn \& Gullahorn, 1963; Tritonovitch,

1977; Ward, Okura, Kennedey \& Kojima (1998)

hier auch Entscheidungs- und Aufgabenmodelle (siehe Schock- und Stressmodelle)

Kommuni-
Kationsan-
sätze
$(4.7)$

Linguistische Assimilation, Adaptation und Akkomodation

Theory of Interpersonal Accomodation through Language von Giles, Taylor and

Bourhis (1973; oder Ethnolinguistic Identity Theory nach Giles and Johnson, 1987)

Lingual assimilation and Bilinguality Approach von Hegele und Pommerin (1983)

Adaptive Intercultural Communication Approach nach Ellingsworth (1983)

Lingual Assimilation and Bilinguality Approach Georgoiannis (1985)

Lingual Assimilation and Bilinguality Approach nach Kummer (1990)

Acculturation from the perspective of the natives approach nach Kim und Berry (1993)

Communication Accomodation Theory (CAT) nach Gallois, Giles, Cargile und Ota (1995)

Linguistic Acculturation nach Epstein, Botvin Dusenberry, Diaz und Kerner (1996)

Authentic Intercultural Communication and Adaptation Approach nach Fox (1997)

Non-linear Model of Linguistic Acculturation and Ethnic Identification von Laroche,

Kim, Hui und Tomiuk (1998)

Integrative Theory of Communicationand Cross-Cultural Adaptation von Y.Y. kim (2001)

Kommunikation im intergruppalen Kontext

Theory of Assimilation, Deviance and Alienation States in Intercultural Communication von McGuire und McDermott (1988)

Theory of Intercultural Identity in Interactions von Collier und Thomas (1988)

Identity Management Theory von Cupach und Imahori (1993)

Identity Negotiation Theory von Ting-Toomey $(1993,1999)$

Co-Cultural Theory (of Communication and Dominance) von Orbe (1998 a/b) 


\begin{tabular}{lll}
\hline Netzwerk- & Persönliche Netzwerke & Akkulturatio \\
Theorien & Personal Network Approach von Y.Y. Kim (1986) & durch soziale \\
$(4.8)$ & Kulturelle Netzwerke & Unterstützun \\
& Intracultural and Intercultural Network Approach von Yum (1988) & g (Kontakt, \\
& Intracultural Network Theory von Smith (1999) & Partizipation
\end{tabular}

Gruppen Passungs- und Identitätsmodelle

Bezogene Mobility Model of Cultural Integration nach Moghaddam et al. (1988; Moghaddam \&

Modelle Taylor, 1987; Moghaddam, Taylor \& Lalonde, 1987; Moghaddam, Taylor \& Wright,

(4.9) 1993) (Assimilation versus Kulturverhaftung $X$ Annahme von normativen versus nicht normativem Verhalten)

Normative Fit-Model von Nesdale (2002)

Sozial-kognitives Modell der Akkulturation nach Padilla und Perez (2003)

Gruppenbildung

Ethnic Minority Formation Approach nach Van Amersfoort ( 1982; Van Amersfoot \&

Surie, 1987)

Attitudes, Represantations, Ideology Approach nach Simpson und Yinger (1986)

$\begin{array}{ll}\text { Re-entry und } & \text { Rückkehr } \\ \text { Re-Migration } & \text { Devlopmental Model of Re-entry Shock von Hertz (1984 }\end{array}$

(4.10) Acculturation Process of Re-entry Approach nach Martin (1984)

Identity and Re-entry Approach von Isogai, Hayashi und Uno (1999)

Integrative Theory of Communication and Cross-Cultural Adaptation von Y.Y. Kim (2001) (s.o.)

Adaptation

durch und in

Gruppen

(Mitgliedschaf

t, Identität, Fit

der Normen)

(Verortung,

Entbindung)

Übergang

Cultural Transition Cycle nach Sussman $(2000,2001,2002)$

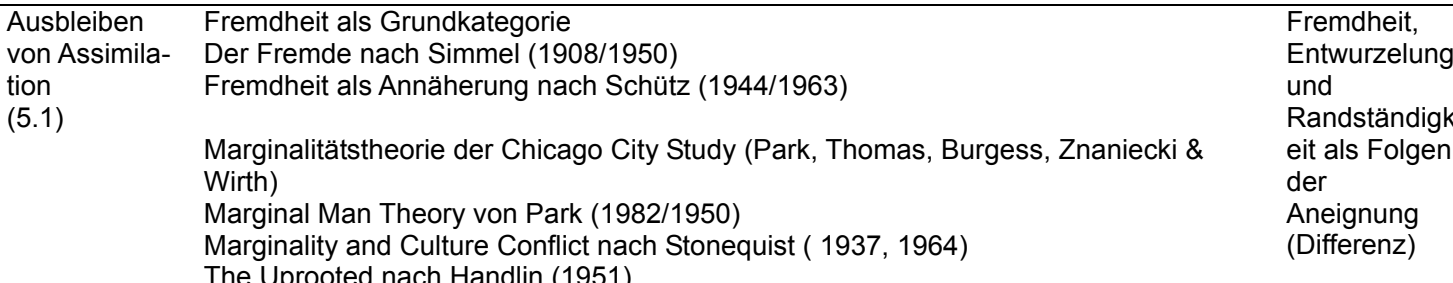

Marginality and Culture Conflict nach
The Uprooted nach Handlin (1951)

Rückkehr als besonderer

Akkulturation sprozess

(Zeit,

Verortung)

(Differenz) 
Gesetzes- $\quad$ Laws of Migration nach Ravenstein (1889) (Klassifikation von Wanderertypen)

und Theory of Migration von Lee (1966/1972) (Push and Pull Factors; Job Vacancy-

Stufen- Hypothesis

Modelle Income-Differential-Hypothesis, Migrant-Stock-Variable Hypothesis)

$(5.2)$

US-amerikanische Assimilationsmodelle

Race Relation Cycle (RRC) von Park und Burgess (1921) (Kontakt, Wettbewerb,

Akkomodation, Assimilation)

Modifikation des RRC zum 7-Stufen Modell durch Bogardus (1929/1930) (Neugierde

der Einheimischen, Ökonomische Eingliederung, Wirtschaftliche und soziale

Antagonismen, Gesetzliche Antagonismen, Humanitäre Gegenbewegungen,

Beruhigung, Schwierigkeiten der zweiten Generation.)

Theory of Absorption oder Model of Partial Assimilation von Eisenstadt (1954) (needs and disposition, physical transition, absorption)

Stage Model of Assimilation von Richardson (1957) (Isolation, Accomodation,

Identification)

Stage-Model of 'Assimilation in American Life von Gordon (1964) (cultural or behavioral, assimilation, marital, identificative, attitude receptional, behavioral receptional, civil)

Stufenmodelle der ,Gastarbeiter-Migration' (in Deutschland)

These der Integration als Teilhabe an Statusstruktur versus Assimilation als

Angleichung an die Kultur von Hoffman-Nowotny (1973, 1987)

Phasenmodell der Akkulturationsprozesses deutscher Einwanderer in Chile von

Waldmann (1982)

(Abschottung, Rekonstruktion, Entfremdung, Selektiver Instrumentalismus, Kulturelle Mischformen)

Verhaltenstheorie der Assimilation und Integration von Einwanderern, ethnischen Gruppen und Minderheiten (oder Rational Choice Theorie der Assimilation und ethnische Differenzierung) von Esser (1980, 1988, 1990) (cognitive, structural, social, identificative assimilation /Akkulturation, Assimilation, Integration)

Assimilation und ethnische Identifikation nach Trube (1984)

These der Binnenintegration durch Pressure Groups von Elwert $(1982,1989)$

Modell der Selbst-Ethnisierung von Apitzsch (2001)

\begin{tabular}{ll}
\hline Identitäts & Grenz-Identität \\
Theorie & Borderland Identity Theory von Laive und Swedenburg (1996)
\end{tabular}

(5.3)

Moving and Mixing

Post-Colonial Theory (of Moving and Mixing) von Bathia und Ram (2001)

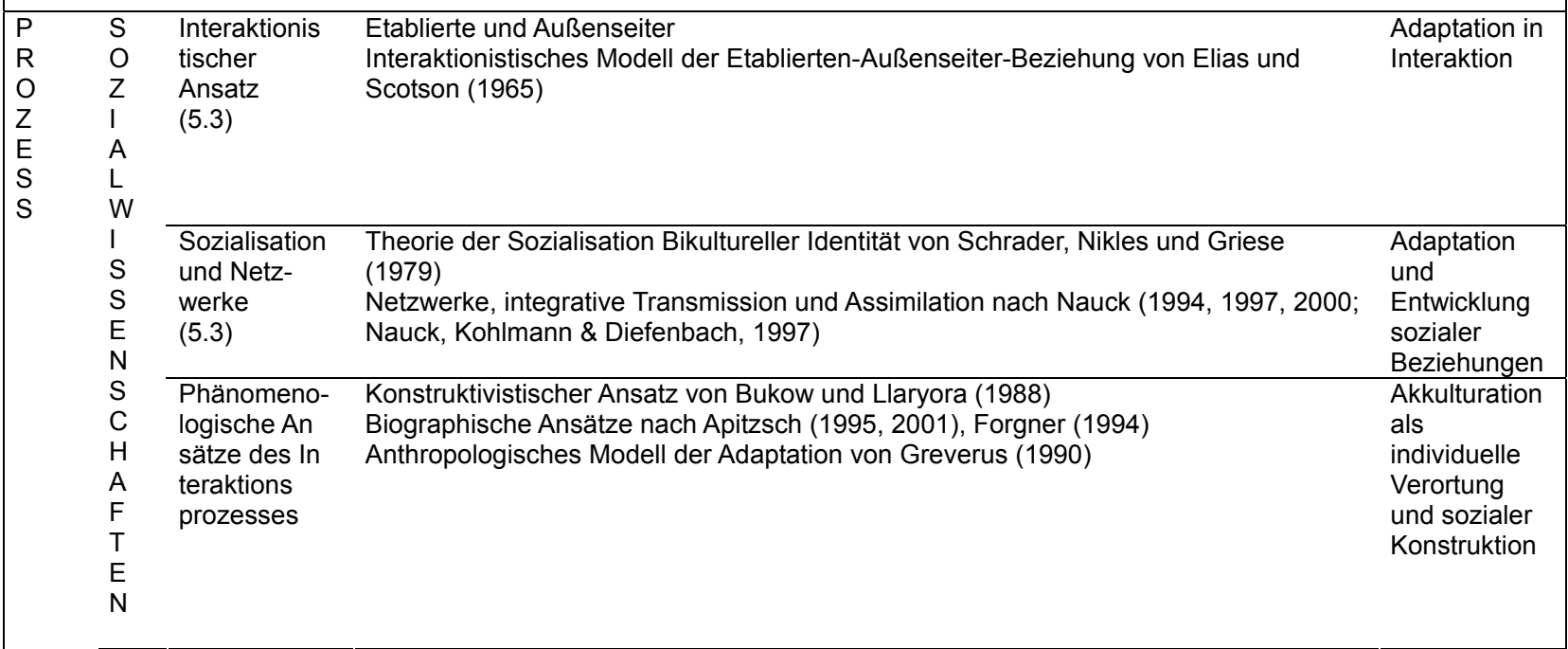


Desintegrati- $\quad$ Theorie der sozialen Desintegration von Anhut und Heitmeyer (2000) (individuellonsthese funktionale, kommunikativ-integrative und kulturell-expressive Sozialintegration)

Akkulturation

(5.3)

Migranten

Migranten-Typologien

und Person

(6.1)

Identitäts

Mobilitätszentrierte Persönlichkeit nach Jennings (1970)

struktur

Innovations- versus Konservationstypen nach Petersen (1972)

(6.2)

(1978)

Theorie der chronisch mobilen Menschen nach Morrison und Wheeler (1978)

The Migrant-Personality nach Boneva und Frieze (2001)

Migranten-Persönlichkeit

Kontrolltheoretischer Ansatz (learned helplessnes, dissonance, loss control) von Masumbuku (1985)

Psychologie der Auswanderung (Lüthke, 1989)

Modell der auswanderungsbereiten Persönlichkeit von Cropley und Lüthke (1994)

Ökologisches Modell der Entwicklungsaufgaben von Ruddat (1994)

im Kontext

sozialer

Integration

und

Systemintegr ation

Akkulturation in

Abhängigkeit

von

Persönlichkeit sprofilen und Dispositionen Adaptation

als

Identitätserha It

Identitätswec hsel und Identitätsgewi $\mathrm{nn}$

Dispositionen und Akkulturation

Extraversion und Sensitivität (Gardner, 1962)

Personal Space (Roger \& Mjoli, 1976)

Locus of Control (Ward \& Kennedey, 1992)

Need for Cognitive Closure (Kosic, 2002)

Basic Social Skills (Hammer, 1987; Argyle, 1969; Furnham, 1979)

Psychological Differentiation (Witkin \& Berry, 1975)

Persönliche Flexibilität, Ambiguitätstoleranz Hardiness, Mastery, Self Efficacy, Self-

Monitoring (vgl. Kim, 2001; Ward, Bochner \& Furnham, 2001)

Big Five (Neuroticsm, Extraversion, Openness, Agreeableness \& Conscientiousness)

(Leong, Ward \& Low, 2000)

Attributionale Komplexität (Stephan \& Stephan, 1992)

Autoritarismus, Rigidität, Ethnozentrismus (Locke \& Feinsod, 1982); Autoritarismus und Dogmatismus (Taft \& Steinkalk, 1985)

Cultural Fit Hypothesis (of Personality and Adaptation) von Searle und Ward (1990;

Ward \& Kennedey, 1993b; Ward \& Chang, 1997)

Identität als orthogonal unabhängige Dimensionen

Bicultural Involvement Model von Szapocznik, Scopetta, Kurtines und Arandale (1978)

Modell der Persistenz ethnischer Identität von Keefe und Padilla (1987)

Orthogonale Kulturelle Identifikationstheorie von Oetting und Beauvais (1990-1991)

Multifaceted Model of Ethnic Identity von Bernal, Knight, Garza, Ocampo und Coxta

(1990; Bernal \& Knight, 1993)

Orientierungs- und Akzeptanz-Typologie nach Montogomery (1992)

Orthogonales Identitätsmodell von Cortés, Rogler und Malgady (1994)

Kategoriale Bikulturalismus- und mehrdimensionale Modelle (Akkulturationsvarianten aus der Kombination der Identifikation mit Herkunfts- und Aufnahmekultur)

Modell der Bikulturellen Identitätsstruktur von Zak (1973)

Orthogonales Modell der Akkulturation (Bikulturelle Identität mit Herkunfts- und/oder

Aufnahmekultur) von Lasry \& Sayegh (1992; Sayegh \& Lasry, 1993; in Anlehnung an Zak, 1973)

Identitätsbasierte Varianten der Akkulturation nach Berry (1974a, 1984a, vgl. auch 1994b, 1997, 2001, 2004)

Chinese American Identity Model (Traditionalismus, Marginalität, Bikulturalismus) von

Sue und Sue (1973)

Modell der zwei Identitäten von Der-Karabetian (1980)

Bikulturelles Identifikationsmodell von Bochner (1982)

Biculturalism-Multiculturalism-Model von Ramirez (1984)

Muster Ethnischer Minderheiten-Identifikation von Hutnik (1986)

Modell der McGill-Gruppe (siehe auch Modelle der Akkulturationsorientierung, unten )

Bicultural Identity Model der nach La Fromboise, Coleman und Gerton (1993)

Differenziertes Modell der Akkulturationsvarianten nach Arends-Tóth und van de

Vijver (2003, 2004) (siehe auch unten)

Cultural Transition Modelle

Theory of Optimal Distinctiveness and Multiple Identities in Cultural Transition von

Brewer (1999)

Socially Constructed World View Model von Chiu und Hong (1999) 
\begin{tabular}{ll}
\hline Modelle der & Mobility Model of Cultural Integration nach Moghaddam et al. (siehe oben Gruppen- \\
Akkultura- & Modelle unter Prozesstheorien) und Modell der McGill-Gruppe
\end{tabular}

Tonsorientie- Theory of Acculturation Tendencies(BAT) von Berry (1980b) (Integration, Assimilation,

rung

Separation, Marginalisierung)

(6.3)

Akkulturationsvarianten nach Berry (1997)

Interactive Acculturation Model (IAM) von Bourhis, Möise, Perreault und Senécal

(1997a/b) (Integration, Assimilation, Separation, Minority: Exclusion/ Individualisation; Majority: Anomy/ Individualisation)

Concordance Model of Acculturation (CMA) von Piontkowski, Rohmann und Florack

(2002)

Relative Acculturation Extended Model (RAEM) nach Navas, Garcia, Sanchez, Rojas, Pumares und Fernandez (2005)

Mutual Acculturation Theory nach Wittig und Molina (2000) und Molina, Wittig und

Giang (2004)

Prozessorientierte Akkulturationsorientierung nach Zick und Six (1995)

Traditionalis- Modernismus versus Traditionalismus These von Berry (1980a) und Dawson, Crano mus, Moder- und Burgoon (1996)

nismis- und Factors of Psychological Adjustment and Sociocultural Adaptation nach Ward und

Adaptations Kennedey, 1994)

modelle Model of Psychological and Sociocultural Adaptation von Leung (2001)

(6.4) Five Level Model of the Acculturation of Native Americans von Garrett und Pichette

(2000) (traditional, marginal, bicultural, assimilated, pan traditional)

Akkulturation als Folge und

Ausdruck generalisierte r

Orientierunge n (Ideologien)

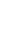




\section{E. Literaturverzeichnis}

Abramson, L.Y., Seligman, M.E.P. \& Teasdale, J.D. (1978). Learned helplessness in humans: Critique and reformulation. Journal of Abnormal psychology, 87, 49-74.

Adamson, F. (2002): Mobilizing for the Transformation of Home: Politicized Identities and Transnational Practices. In: Al-Ali, N., Koser, K. (Hg.): New Approaches to Migration? Transnational Communities and the Transformation of Home, London: Routledge, 155-168.

Ahrenholz, B. (2010) Bedingungen des Zweitspracherwerbs in unterschiedlichen Altersstufen. In: Abteilung Wirstschafts- und Sozialpolitik der Friedrich-Ebert-Stiftung (Hrsg.) (2010) „Sprache ist der Schlüssel“. Bedingungen des Sprachlernens von Menschen mit Migrationshintergrund. Bonn = Wiso Diskurs, bin nicht sicher ob der Titel das zum Titel gehört. http://library.fes.de/pdf-files/wiso/07666.pdf

Al-Ali, N., Koser, K. (Hrsg.)(2002). New Approaches to Migration? Transnational Communities and the Transformation of Home, London: Taylor \& Francis Books.

Al-Barghouti, G. (2001): Erste Erfahrungen aus den Probestandorten. In: Älter werden in Deutschland. Fachtagung zu einer Informationsreihe für ältere Migranten (2001), Hrsg. von der Beauftragten der Bundesregierung für Ausländerfragen, Berlin.

Angel, R.J. Angel, J.L., Venegas, C.D., Bonazzo, C. (2010). Shorter Stay, Longer Life: Age at Migration and Mortality Among the Older Mexican-Origin Population. In: Journal of Aging and Health 22 (7): 914-931, Thousand Oaks: Sage Publications.

Angenendt, S., Kruse, I. (2003). Der schwierige Wandel. Die Gestaltung der deutschen und europäischen Migrationspolitik an der Wende vom 20. zum 21. Jahrhundert. In: Oltmer, J. (Hrsg.). Migration steuern und verwalten. Deutschland vom späten 19. Jahrhundert bis zur Gegenwart (S. 481-497). Göttingen: V\&R unipress.

Arbeiterwohlfahrt - Abschlußbericht zum Projekt „Aufbau einer Altentagesstätte für türkische Seniorinnen und Senioren“. Oktober 1993 bis Oktober 1996

Arbeitsgemeinschaft der Wissenschaftlichen Medizinischen Fachgesellschaften. AWMF online. http://www.awmf.org/uploads/tx_szleitlinien/038-

015_S2_Persoenlichkeitsstoerungen_05-2008_05-2013.pdf, letzter Zugriff: 14.06.2011.

Aristoteles, Peri hermeneias, Erstes Kapitel

Atabay, I. (2010). Ich bin Sohn meiner Mutter, Elterliches Bindungsverhalten und männliche Identitätsentwicklung in türkeistämmigen Familien. Freiburg: Centaurus.

Auernheimer, G. (1988): Der sogenannte Kulturkonflikt. Orientierungsprobleme ausländischer Jugendlicher. Frankfurt a.M., New York: Campus.

Auernheimer, G. (2007). Einführung in die Interkulturelle Pädagogik. Darmstadt: WBG.

Ausländerbeauftragte der Bundesregierung. (2001).

http://www.destatis.de/jetspeed/portal/cms/Sites/destatis/Internet/DE/Content/Publik ationen/Fachveroeffentlichungen/Bevoelkerung/Migration/ntegration/AuslaendBevoe Ikerung2010200107004, property=file.pdf, aufgerufen am 26.05.2011. In: Migrationsbericht der Ausländerbeauftragten im Auftrag der Bundesregierung bezogen von http://www.bmi.bund.de/cae/servlet/contentblob/149602/publicationFile/15184/migrat ionsbericht_2001.pdf, letzter Zugriff: August 2007. 
Bade, K.J. (1994b). Ausländer - Aussiedler - Asyl: eine Bestandsaufnahme. München: Beck.

Bade, K.J. (1996). Transnationale Migration, ethnonatiomale Diskussion und staatliche Migrationspolitik im Deutschland des 19. und 20. Jahrhunderts. In: Bade, K.J. (Hrsg.), Migration - Ethnizität - Konflikt: Systemfragen und Fallstudien (S. 403-430). Osnabrück: Institut für Migrationsforschung und Interkulturelle Studien (IMIS).

Bade, K.J. (Hrsg.) (1994a). Das Manifest der 60: Deutschland und die Einwanderung. München: Beck.

Bade, K.J. (Hrsg.) (1997). Fremde im Land: Zuwanderung und Eingliederung im Raum Niedersachsen seit dem Zweiten Weltkrieg. Osnabrück: Institut für Migrationsforschung und Interkulturelle Studien (IMIS).

Bandura, A. (1977). Self-efficacy: Toward a unifying theory of behavioral change. Psychological Review, 84, 191-215.

Bandura, A. (1981). Soziale Unterstützung und chronische Krankheit. Frankfurt a. M.: Suhrkamp.

Bandura, A. (1998). Health promotion from the perspective of social cognitive theory. Psychology and Health, 13: 623-649.

Batchelder, D. (1977). Developing Cross-Cultural Learning Skills. In Batchelder, D. \& Warner, E.G. (Hrsg.). Beyond Experience - The Experiental Approach to CrossCultural Education (P.148f). Maine: Intercultural Press.

Bauer, J. (2007). Das Gedächntis des Körpers: Wie Beziehungen und Lebensstile unsere Gene steuern. Frankfurt: Piper.

Baykara-Krumme, H., Hoff, A. Die Lebenssituation älterer Ausländerinnen und Ausländer in Deutschland. In: Tesch-Römer, C. et al. $(\mathrm{Hg})$ : Altwerden in Deutschland. Sozialer Wandel und individuelle Entwicklung in der zweiten Lebenshälfte (S. 447f). Wiesbaden 2006.

Beck-Gernsheim, E. (2004). Wir und die Anderen: vom Blick der Deutschen auf Migranten und Minderheiten . Frankfurt am Main: Suhrkamp.

Bericht der Medienkommision im Bundesverein (2001). Nachtlicht - „Ein Koffer gegen Kindesmisshandlung“!? In: prävention - Zeitschrift des Bundesvereins zur Prävention von sexuellem Missbrauch 2001 Jahrgang 4 Heft 1, 19-21.

Berry, J. W . (1974). Psychological aspects of cultural pluralism: Unity and identity reconsidered. Topics in Cultural Learning, 2, 17-22.

Berry, J. W. (1980). Acculturation as varieties of adaptation. In A. M. Padilla (Ed.), Acculturation: Theory, models, and some new findings (pp. 9-25). Boulder, CO: Westview.

Berry, J.W., Phinney, J.S., Sam, D.L. Vedder P. (2006). Immigrant Youth: Acculturation, Identity, and Adaptation. Applied Psychology: An international Review, 55 (3), 303332.

Beutel, M. E., Decker, O., Brähler, E. (2007). Welche Auswirkungen haben Flucht und Vertreibung auf Lebensqualität und Befindlichkeit? Repräsentative Erhebung mit den vor 1946 Geborenen in Deutschland. In: Zeitschrift für Psychosomatische Medizin und Psychotherapie 53, $204-215$. 
Bhabha, H. K. (2000). Die Verortung der Kultur. (Aus dem Englischen übersetzt von: Schiffmann, M. und Freudl, J.) (The location of culture (engl. 1994). Tübingen: Stauffenburg Verlag.

Bohner, G., Siebler, F., González, R., Haye, A., Schmidt, E. A. (2008). Situational flexibility of in-group-related attitudes: A single category IAT study of people with dual national identity. Group Processes and Intergroup Relations, 11, 301-307.

Bommes, M. (1997). Von ıGastarbeitern ‘ zu Einwanderern: Arbeitsmigration in Niedersachsen, in: K.J. Bade (Hg.). Fremde im Land: Zuwanderung und Eingliederung im Raum Niedersachsen seit dem Zweiten Weltkrieg. IMIS-Schriften 1997 Bd. 3, S. 249-322.

Boos-Nünning, U, (1994). Familie, Jugend, Bildungsarbeit. In: Bade, K.J. (Hrsg.), Das Manifest der 60: Deutschland und die Einwanderung. München: Beck.

Boos-Nünning, U. (1998). Arbeiten und Wohnen als Lebensgrundlage. In: Eryılmaz, A. und Jamin, M. (Hrsg.). Fremde Heimat (S. 337-357). Essen: Klartext Verlag.

Bourhis, R. Y., Moise, L. C.,Perreault, S., Senecal, S. (1997). Towards an interactive acculturation model: A social psychological approach. In: International Journal of Psychology, 32 (6): 369-386.

Bower, G. H., Hilgard, E. R. (1983). Theorien des Lernens. Stuttgart: Klett-Cotta Verlag.

Bradley, D.E., Van Willigen, M. (2010). Migration and Psychological Well-Being Among Older Adults: A Growth Curve Analysis Based on Panel Data From the Health and Retirement Study, 1996-2006 In: Journal of Aging and Health 22 (7): 882-913.

Brand, M., Markowitsch, H.J. (2009). Lernen und Gedächtnis aus neurowissenschaftlicher Perspektive, In: Hermann, U. (Hrsg.), Neurodidaktik, Grundlagen und Vorschläge für gehirngerechtes Lernen, 2. Aufl., Beltz: Weinheim/Basel.

Brandrup-Lukanow A. (2000). Reproduktive Gesundheit - die Perspektive der WHO. In: Familienplanung und Lebenslaeufe von Frauen- Kontinuitaeten und Wandel, Dokumentation der Bundeszentrale für gesundheitliche Aufklärung. (BzgA)(2000).

Braun, S., Kling, C., Vey, A.L. (2001). Aktuelle Erprobung des neuen Medienkoffers „Älter werden in Deutschland“ am Beispiel der Kreisverbände des Deutschen Roten Kreuzes in Reutlingen, Stuttgart und Tübingen. In: Älter werden in Deutschland. Fachtagung zu einer Informationsreihe für ältere Migranten, Nr. 11. Berlin/Bonn, S. 68-86.

Brettel, C. (2000). Theorizing Migration in Anthropology: The Social Construction of Networks, Identities, Communities, and Globalscapes. In: Brettel, C.B. und Hollifield, J.F.. Migration Theory: Talking across Disciplines. New York London: Routledge.

Brettel, C.B., Hollifield, J.F. (2000). Migration Theory: Talking across Disciplines. New York, London: Routledge.

Brooker, P. (2003). A Glossary of Cultural Theory. New York :Bloomsbury Academic.

Büchel, F., Pannenberg, M. (2004). Berufliche Weiterbildung in West- und Ostdeutschland. Teilnehmer, Struktur und individueller Ertrag. Zeitschrift für Arbeitsmarkt Forschung, 37 (2), S. 73-126.

Bühler, K. (1978)(Erstausgabe 1934). Sprachtheorie. Die Darstellungsfunktion der Sprache. Frankfurt/Berlin/Wien: Ullstein. 
Bukow, W.-D. (2010). Urbanes Zusammenleben - Zum Umgang mit Migration und Mobilität in europäischen Stadtgesellschaften. Wiesbaden: VS Verlag für Sozialwissenschaften.

Bükrücü, I. (2001). Alte Migrantinnen und Migranten als Kunden der ambulanten Pflege. In: Kaewnetara, E., Uske, H. (Hrsg.): Migration und Alter. Auf dem Weg zu einer kulturkompetenten Altenarbeit. Konzepte - Methoden - Erfahrungen. Duisburg: Unrast-Verlag.

Bundesamt für Migration und Flüchtlinge (2005) Der Einfluss von Zuwanderung auf die deutsche Gesellschaft. Nürnberg: Bundesamt für Migration und Flüchtlinge. http://www.integrationsportal.nrw.de/wissenschaft/Studien/Migrationsberichte_EU_B und_Land/Einfluss_von_Zuwanderung/index.php, letzter Zugriff: 13.03.2011.

Bundesamt für Migration und Flüchtlinge (2010). Fortschritte der Integration: Zur Situation der fünf größten in Deutschland lebenden Ausländergruppen. Nürnberg: Bundesamt für Migration und Flüchtlinge. http://www.integration-in-

deutschland.de/nn_441298/SharedDocs/Anlagen/DE/Migration/Publikationen/Forsc hung/Forschungsberichte/fb8-fortschritte-der-integration.html, letzter Zugriff Mai 2011.

Bundesamt für Migration und Flüchtlinge (BAMF) (2011). Das Bundesamt informiert: Zahlen, Daten und Fakten zu den Integrationskursen. http://www.integration-indeutschland.de/nn_442622/SharedDocs/Pressemitteilungen/DE/DasBAMF/2010/10 0928-0017-pressemitteilung-ik-zahlen.html, letzter Zugriff: 16.06.2011.

Bundesamt für Migration und Flüchtlinge (BAMF) (2010). Präsentationen der Sektionstagung "Viele Welten des Alterns? Ältere Migrantinnen und Migranten im alternden Deutschland" (in Kooperation mit dem DZA und dem BAMF) am 24. und 25. Juni 2010 in Berlin. http://www.sektion-altern.de/de/praes_st_2010.htm, letzter Zugriff: 15.06.2011.

Bundesamt für Migration und Flüchtlinge (BAMF). (2010). Statistik zu den bundesweiten Integrationskurszahlen.

http://www.bamf.de/cln_103/DE/Infothek/Statistiken/InGe/DatenBund/daten-bundnode.html, letzter Zugriff: 15.06.2011.

Bundeszentrale für politische Bildung (2005a). Von der "Gastarbeiter"-Anwerbung zum Zuwanderungsgesetz Migrationsgeschehen und Zuwanderungspolitik in der Bundesrepublik Reißlandt, C.

http://www.bpb.de/themen/6XDUPY,5,0,Von_der_GastarbeiterAnwerbung_zum_Zu wanderungsgesetz.html\#art5, letzter Zugriff: 01.08.2010.

Bundeszentrale für politische Bildung (2005b). Migration in Ost- und Westdeutschland von1955 bis 2004.

http://www.bpb.de/themen/8Q83M7,0,0,Migration_in_Ost_und_Westdeutschland_vo n_1955_bis_2004.html. Letzter Zugriff: 01.08.2010.

Bündnis 90 - Die Grünen Bundestagsfraktion (2008). Erwachsenen-BAföG. Eckpunkte für ein Bundesgesetz zur Förderung der individuellen Fort- und Weiterbildung. http://www.priska-hinz.de/cms/default/dok/270/270278.erwachsenenbafoeg.html, Zugriff am 07.08.2009.

Busch, A., Stenschke, O. (2007). Germanistische Linguistik. Tübingen: Narr.

Calandreau L, Bertin A, Boissy A, Arnould C, Constantin P, Desmedt A, Guémené D, Nowak R, Leterrier C. (2010). Effect of one week of stress on emotional reactivity and learning and memory performances in Japanese quail. In: Journal of Behavioral Brain Research 2011 2; 217(1):104-110. 
Calicoglu, M., Müller F., Wallraff, A. (2001). Neue Wege einer offenen multikulturellen Seniorenarbeit. Das Stadtteilprojekt Bochum Dahlhausen, in: Kaewnetara, E.. Uske, $\mathrm{H}$. (Hrsg.): Migration und Alter. Auf dem Weg zu einer kulturkompetenten Altenarbeit. Konzepte - Methoden - Erfahrungen (S. 89 - 116). Duisburg: UnrastVerlag.

Canning, C. M. (2005). The most American thing in America. Circuit Chautauqua as performance. lowa City: University of lowa Press.

Cannon, W.B. (1915 rev. Auflage 1929). Bodily Changes in Pain, Hunger, Fear and Rage. New York: Appleton.

Cannon, W.B. (1935). Stresses and strains of homeostasis. The American Journal of the Medical Sciences, 189(1): 1-14.

Ceylan, R. (2006). Ethnische Kolonien. Wiesbaden: VS Verlag

Cheka, G., Knipscheer, J., Kleber, R. (2010). The Impact of Forced Migration on Health: A Comparative Study on Posttraumatic Stress Among Internally Displaced and Externally Migrated Kurdish Women. In: Traumatology December 2010 vol. 16 no. 4: 109-116.

Conway, K., Houtenville, A.J. (2001). Elderly migration and state fiscal policy: evidence from the 1990 census migration flows. In: National Tax Journal Vol. 54: 103-124.

Cusworth, R. A. (1995). The Framing of Educational Knowledge through „Show and Tell“ in Elementary Classrooms. Forschungspaper zum „Annual Meeting of the American Educational Research Association“; 18.-22. April 1995.

D’Amato, G. (2005). Vom Ausländer zum Bürger. Der Streit um die politische Integration von Einwanderern in Deutschland, Frankreich und der Schweiz (3. Aufl.). Münster: Lit Verlag.

de Saussure, F. (2001). Grundfragen der allgemeinen Sprachwissenschaft. Berlin/New York: deGryter Studienbuch.

Deutsche Islam Konferenz (DIK). www.deutsche-islam-konferenz.de, letzter Zugriff: 16.06.2011.

Deutscher Bundestag Berlin 2002: 142

Deutscher Bundestag (2002). 14. Wahlperiode, Drucksache 14/9883. Unterrichtung durch die Beauftragte der Bundesregierung für Ausländerfragen - Bericht über die Lage der Ausländer in der Bundesrepublik Deutschland, Berlin.

Deutscher Verein für öffentliche und private Fürsorge e.V. (2010) Empfehlungen des Deutschen Vereins zur besseren Teilhabe älterer Menschen mit Migrationshintergrund Seite 3-4. http://www.familienbotschaftmv.de/fileadmin/Newsletter/DV_33-10_Empfehlungsschreiben.pdf, letzter Zugriff: 15.06.2011.

Deutsches Rotes Kreuz (2010). http://www.aelter-werdenin.de//index.php?option=com_content\&task=view\&id=54\&ltemid=181, letzter Zugriff: 15.06.2011.

Diehl, M., Hay, E. (2010). Reactivity to daily stressors in adulthood: The importance of stressor type in characterizing risk factors. In: Psychology and Aging, Vol 25(1), Mar 2010: 118-131. 
Dohmen, G. (2001). Das informelle Lernen - Die internationale Erschließung einer bisher vernachlässigten Grundform menschlichen Lernens für das lebenslange Lernen aller. Bonn: Bundesministerium für Bildung und Forschung (BMBF) Referat Öffentlichkeitsarbeit.

Dörpinghaus, A., Poenitsch, A., Wigger, L. (2008). Einführung in die Theorie der Bildung. Darmstadt: WBG.

Ebner, G. (2001). Grundlagen transkultureller Begutachtung aus klinischer Sicht Gütekriterien und Ausblick. In: Migrationsspezifische Begutachtung im Spannungsfeld von Medizin, Recht Psychologie und Politik S. 34. Fachtagung Hannover, 2./3. Nov. 2001.

Encarta (2009). Suchbegriff "Antike". Microsoft® Encarta® Online-Enzyklopädie 2009. 1997-2009 Microsoft Corporation. Zugriff am 28.07.2009, von http://de.encarta.msn.com/encyclopedia_721534000/Antike.html.

Eppinger, B., Kray, J. (2010). To choose or to avoid: Age differences in learning from positive and negative feedback. Journal of Cognitive Neuroscience,23: $41-52$.

Erdmann, G., Baumann, S. (1996). Sind psychophysiologische Veränderungen im Paradigma „Öffentliches Sprechen“ Ausdruck emotionaler Belastungen? Zeitschrift für Experimentelle Psychologie, XLIII: 224-255.

Ertl, A. (2001) Kultursensible Pflege - das Nadelöhr zu einer individuellen Pflege für alle (117-137); Anne Dietrich: Die Alten sind doch alle gleich! Oder? Interkulturelle Kompetenz im Gesundheits- und Altenpflegebereich. In: Kaewnetara, E. und Uske, $\mathrm{H}$. (Hrsg.): Migration und Alter. Auf dem Weg zu einer kulturkompetenten Altenarbeit. Konzepte - Methoden - Erfahrungen (S. 124-150). Duisburg: UnrastVerlag.

Eryılmaz, A. (1998). Das Leben im Wohnheim. In: Eryılmaz, A., Jamin,M. (Hrsg.), Fremde Heimat (S. 171-177) und (S. 93-122). Essen: Klartext Verlag.

Esser, H. (1980). Aspekte der Wanderungssoziologie. Assimilation und Integration von Wanderern, ethnischen Gruppen und Minderheiten. Eine handlungstheoretische Analyse. Neuwied/Darmstadt: Hermann Luchterhand Verlag.

Esser, H. (2001). Integration und ethnische Schichtung. Arbeitspapiere - Mannheimer Zentrum für Europäische Sozialforschung / Nr 40. Mannheim.

Europäischer Rat (2000). Schlussfolgerungen des Vorsitzes. Lissabon.

Expertenkommission Finanzierung Lebenslangen Lernens (2004). Schlussbericht der unabhängigen Expertenkommission Finanzierung Lebenslangen Lernens: Der Weg in die Zukunft. Deutscher Bundestag Drucksache 15/3636, 15. Wahlperiode 03. 08. 2004.

Fach, Chr.. (2001). Materialien \& Medien I: Transfer, Methodik und Didaktik der Informationsreihe, in: Älter werden in Deutschland. Fachtagung zu einer Informationsreihe für ältere Migranten, Nr. 11 (S. 35). Berlin/Bonn.

Faure et al. (1972). Learning to be. The world of education today and tomorrow. Paris: UNESCO.

Fauser, M., (2008). Einführung in die Kulturwissenschaften. Darmstadt: WBG.

Flechsig, K.-H., Haller H.-D. (1975). Einführung in didaktisches Handeln. Ein Lernbuch für Einzel- und Gruppenarbeit. Stuttgart. Klett. 
Flechsig, K.-H., Haller H.-D. (2003). Einführung in Didaktisches Handeln Ein Lernbuch für Einzel- und Gruppenarbeit. Stuttgart 1975 online Veröffentlichung gkdm website Vorwort zur Internet-Ausgabe 2003.

Flechsig, K.-H. (1996). Das "Kleine Handbuch Didaktischer Modelle", Eichenzell: Neuland-Verlag für lebendiges Lernen.

Flechsig, K.-H. (2009). Antizipatorisches Lernen mit Planspielen. In: Bosse, D., Posch, P. (Hrsg.), Schule 2020 aus Expertensicht.- Zur Zukunft von Schule, Unterricht und Lehrerbildung.( S. 155-161). Wiesbaden: VS Verlag.

Freire, P. (1973). Pädagogik der Unterdrückten. Bildung als Praxis der Freiheit. Hamburg: Reinbek

Freire, P. (1983). The importance of the act of reading. Journal of Education, v165 n1: 511.

Frese, M., Zapf, D. (1988). Methodological Studies in the Study of Work Stress: Objective vs. Subjective Measurement and the Question of Longditudinal Studies. In: Cooper, C.L., Payne, R. (Hrsg.), Causes, Coping and Consequences of Stress at work (S. 375-411). Chichester: Wiley.

Frese, M. (1989). Theoretical Model of contorl and health. In: Sauter, S.L., Hurrel, J.J., Cooper, C.L. (Hrsg.), Job Control and Worker Health (p 107-127). London: Wiley.

Frewer W., Jäger, K. (2001). Interkulturelle Kompetenz in der Aus- und Weiterbildung. Erfahrungen aus einem transnationalen Projekt im Bereich (Alten-)Pflege. In: Kaewnetara, E., Uske, H. (Hrsg.), Migration und Alter. Auf dem Weg zu einer kulturkompetenten Altenarbeit. Konzepte - Methoden - Erfahrungen (S. 39-55). Duisburg: Unrast-Verlag.

Frölich, M. (2003). Migration als biografische und expressive Ressource - Beiträge zur kulturellen Produktion in der Einwanderungsgesellschaft. In: Arnoldshainer Interkulturelle Diskurse. 3; Wissen und Praxis. 118:S. 45-66).

Fuhrken, S. (2008). Parallelgesellschaften? Zum Begriff und der empirischen Untersuchung in NRW. In: Kissau, K., Kortmann, M., Tietze, K., Cordier, C., Alsünger, H., Musch, E. (Hrsg.), Migration steuern oder verwalten? Deutschland im internationalen Vergleich (S.112-120). Tagungsband der Universität Münster.

Gerlach, C. (2000). Lebenslanges Lernen. Konzepte und Entwicklungen 1972 bis 1997. Kölner Studien zur internationalen Erwachsenenbildung. Beihefte zum Internationalen Jahrbuch der Erwachsenenbildung, Band 12: 162-163. Köln: Böhlau..

Glaser, E., Rendtorff, B. (2006). Menschenbilder und Geschlecht - Bildungs- und Erziehungskonzepte in verschiedenen Lebensphasen. In: Fatke, R., Merkens, H. (Hrsg.). Bildung über die Lebenszeit (S. 173-177). Wiesbaden: VS Verl. für Sozialwiss..

Gogolin, I. (Hrsg.)(2000). Migration, gesellschaftliche Differenzierung und Bildung. Opladen: Leske + Budrich.

Goldberger, L, Breznitz, S (Eds) (1993). The Handbook of Stress, Theoretical and Clinical Aspects. New York: Free Press.

Grieger, D. (2001). Soziodemographische Daten und Fakten zur Situation älterer Migrantinnen und Migranten in der Bundesrepublik Deutschland. In: Beauftragte der Bundesregierung für Ausländerfragen (Hrsg.), Älter werden in Deutschland. Fachtagung zu einer Informationsreihe für ältere Migranten (S. 9-17). Berlin. 
Gujons, H. (1995). Pädagogisches Grundwissen: Überblick - Kompendium - Studienbuch. Bad Heilbrunn: Klinkhardt.

Gül, V, Öner, E, Uyar, K, Kultur und Ethnizität - Ihre Rolle bei der Entstehung psychischer Störungen am Beispiel der türkischen Gemeinschaft in Deutschland, psychoneuro 2008; 34(2): 97-99.

Hackhausen, W. (2001). Psychosoziale Bedingtheit in der Sozialmedizin und Rehabilitation. In: Schröder, H., Hackhausen, W. (Hrsg.), Persönlichkeit und Individualität in der Rehabilitation (S.7-27). Frankfurt/Main: VAS.

Hailbronner, K. (2001). Das neue deutsche Staatsangehörigkeitsgesetz. In: Neue Verwaltungszeitschrift, 20: 1329-1335.

Hämmerer, D., Li, S.-C., Müller, V., Lindenberger, U. (2011). Lifespan differences in electrophysiological correlates of monitoring gains and losses during probabilistic reinforcement learning. Journal of Cognitive Neuroscience, 23: 579-592.

Hermann, U. (Hrsg.) (2009). Neurodidaktik, Grundlagen und Vorschläge für gehirngerechtes Lernen, 2. Aufl., Weinheim/Basel: Beltz.

Hill, J., Röhl, M. (2002). Muss sich unser Gesundheitssystem auf die Betreuung von Migranten einstellen? In: Dettmers, C., Albrecht, N.-J., Weiller, C. (Hrsg.), Gesundheit Migration Krankheit (S. 113-121). Bad Honnef: Hippocampus.

Hogg, M.A., Vaughan G. (2008). Social Psychology. Harlow: Prentice Hall.

Hogg, M.A., Abrams, D. (1988). Social identifications: A social psychology of intergroup relations and group processes. London: Routledge.

Holmes TH, Rahe RH (1967). "The Social Readjustment Rating Scale". J Psychosom Res 11 (2): 213-8.

von Holst, D., Fuchs, E., Stöhr, W. (1983). Physiological changes in male Tupaia belangeri under different types of social stress. In: Dembroski, T.M., Schmidt, T.H., Blumchen, G. (Hrsg.), Biobehavioural Bases of Coronary Heart Disease, Vol 2. (pp382-390). Basel: S Karger.

Hörz, P.F.N. (2002). Der Name der Hose. Levi Strauss und die Geschichte der blauen Beinkleider.

http://www.wienerzeitung.at/Desktopdefault.aspx?TabID=3946\&Alias=wzo\&lexikon= Mode\&letter=M\&cob=4901, Zugriff am 06.08.09.

Huddy, L., Khatib N. (2007). American Patriotism, National Identity, and Political Involvement. American Journal of Political Science, 51(1): 63-77.

Hunger, U., Thränhardt, D. (2004). Migration und Bildungserfolg: Wo stehen wir? In: Bade, K.J., Bommes, M. (Hrsg.), Migration - Integration - Bildung. Grundfragen und Problembereiche (S.179-197). Osnabrück: Institut für Migrationsforschung und Interkulturelle Studien (IMIS).

Hurrelmann, K. (2002). Einführung in die Sozialisationstheorie, 8. Aufl. .Weinheim: Beltz Verlag.

Hüther, G. (2005). Über die Kunst, gute Erfahrungen im Gehirn zu verankern, Wiener Medizinische Wochenschrift, 155/23-24: 537- 538.

Hüther, G. (2009). Metakompetenzen und ICH-Funktionen während der Kindheit, In: Hermann, U. (Hrsg.), Neurodidaktik, Grundlagen und Vorschläge für gehirngerechtes Lernen, 2. Aufl. (S. 99-109). Weinheim/Basel: Beltz. 
Hüther, G., Sachsse, U. (2007). Angst- und stressbedingte Störungen, Auf dem Weg zu einer neurobiologisch fundierten Psychotherapie, Psychotherapeut, 52:166-179.

Irle, E., Lange, C., Sachsse, U., Weniger, G. (2009). Further evidence that post-traumatic stress disorder but not dissociative disorders are related to amygdala and hippocampal size reduction in trauma-exposed individuals. Acta Psychiatrica Scandinavica, 119: 330-331.

Jäkel, J., Leyendecker, B. (2008). Tägliche Stressfaktoren und Lebenszufriedenheit türkischstämmiger Mütter in Deutschland. In: Zeitschrift für Gesundheitspsychologie 1:12-21.

Jamin, M. (1998). Die deutsche Anwerbung: Organisation und Größenordnung. In: Eryılmaz, A., Jamin; M. (Hrsg.), Fremde Heimat (S. 149-170). Essen: Klartext Verlag.

Janke W., Wolffgramm, J. (1995). Biopsychologie von Streß und emotionalen Reaktionen: Ansätze interdisziplinärer Kooperation von Psychologie, Biologie und Medizin. In: Debus, G., Erdmann, G., Kallus, K.W, Biopsychologie von Streß und emotionalen Reaktionen (S. 293-347). Göttingen: Hogrefe.

Jerusalem, M., Schwarzer, R. (1992). Self-efficacy as a Resource Factor in Stress Appraisal Prozesses. In R. Schwarzer (Ed.), Self-efficacy: Thought Control in Action. Washington: Hemisphere Publishing.

Kalter, F, Granato, N. (2004). Sozialer Wandel und strukturelle Assimilation in der Bundesrepublik. Empirische Befunde mit Mikrodaten der amtlichen Statistik. In: Bade, K.J., Bommes, M. (Hrsg.), Migration - Integration - Bildung: Grundfragen und Problembereiche. Osnabrück: Institut für Migrationsforschung und Interkulturelle Studien (IMIS).

Kasl, S.V., Cooper, C. L. (Hrsg.) (1987). Stress and Health: Issues in Research Methodology. Chichester: John Wiley.

Kasl, S. (1996). Influence of the work environment on cardiovascular health: a historical, conceptual and methodological perspective. Journal of Occupational Health Psychology, 1: 42-56.

Keim, S. (2003). "So richtig deutsch wird man nie sein - ": junge Migrantinnen und Migranten in Deutschland : zwischen Integration und Ausgrenzung. Berlin: IKO Verlag.

Kell, A. (1996). Lebenslanges Lernen - aus historischer Sicht. Die Berufsbildende Schule, 48 (2): 48-56.

Keskin, H. (1998). Die Richtung ist entscheidend. In: Eryılmaz, A., Jamin, M. (Hrsg.), Fremde Heimat (S. 19-22). Essen: Klartext Verlag.

Kirschbaum, C., Pirke, K. M., Hellhammer, D. H. (1993). The 'Trier Social Stress Test' --a tool for investigating psychobiological stress responses in a laboratory setting. Neuropsychobiology 28(1-2): 76-81.

Kolb, H. (2004): Einwanderung zwischen wohlverstandenem Eigeninteresse und symbolischer Politik. Das Beispiel der deutschen „Green Card“. Studien zu Migration und Minderheiten 12. Münster: LIT.

Kommission der Europäischen Gemeinschaften (2000). Memorandum über Lebenslanges Lernen. Arbeitsdokument der Kommissionsdienststellen. Brüssel. 
Kulischer, J. (1988). Allgemeine Wirtschaftsgeschichte des Mittelalters und der Neuzeit, Band 1. Oldenbourg: Wissenschaftsverlag.

Kurtoğlu, I. (2008). Und die Türken kommen - nicht!. Göttingen: Selbstverlag.

Landwehr, A. (2007). Wissensgeschichte. In: Rainer Schützeichel (Hg.): Handbuch Wissenssoziologie und Wissensforschung. Konstanz: UVK.

Lazarus, P., Homer, S. L., (1980). Sharing Time in Kindergarten: A Study of the Relationship between Structure and Content. Forschungspaper zum „Annual Meeting of the Rocky Mountain Educational Research Association“; 15.-17. Oktober 1980.

Lazarus, R. S. (1966). Psychological stress and the coping process. New York: McGrawHill.

Lazarus, R. S., Folkman, S. (1984). Stress, appraisal and coping. New York: Springer.

Learnmax (2011). http://www.learnmax.in/Multicultural-Psychology/162.html, letzter Zugriff am 15.06.2011.

Leiprecht, R. und Vogel, D. (2007). Transkulturalität und Transnationalität als Herausforderung für die Gestaltung Sozialer Arbeit und sozialer Dienste vor Ort. In: Homfeldt, H.G., Schröer, W., Schweppe, C. (Hg.). Soziale Arbeit und Transnationalität - Herausforderungen eines spannungsreichen Bezugs, Weinheim

Lengrand, P. (1972). Permanente Erziehung. Eine Einführung. München-Pullach: Dokumentation.

Leyendecker, B. (2003). Frühe Entwicklung im soziokulturellen Kontext (S. 381-431). In: Keller, H. (Hrsg.), Handbuch der Kleinkindforschung. Bern: Huber.

Lindeman, E. C. (1926). The Meaning of Adult Education. New York: New York Republic.

Linehan, M.M. (1993). Cognitive Behavioural Treatment for Borderline Personality Disorder. New York: Guilford Publications

Liotti, M., Panksepp, J. (2004). Imaging Human Emotions And Affective Feelings: Implications For Biological Psychiatry (p. 33-74). In: Panksepp, J. (Hrsg.), Textbook of Biological Psychiatry. Hoboken: Wiley-Liss.

Margolis, M. (1995). Transnationalism and Popular Culture: The Case of Brazilian Immigrants in the United States. Journal of Popular Culture 29: 29-41. Nach Brettel, C. (2000). Theorizing Migration in Anthropology: The Social Construction of Networks, Identities, Communities, and Globalscapes. In: Brettel, C.B. und Hollifield, J.F. (2000). Migration Theory: Talking across Disciplines. New York / London: Routledge.

Marschalck, P. (1997). Bevölkerung und Wanderung im Raum Niedersachsen seit dem Zweiten Weltkrieg. In: K. J. Bade (Hrsg.), Fremde im Land. Zuwanderung und Eingliederung im Raum Niedersachsen seit dem Zweiten Weltkrieg (S. 45-75), Osnabrück: Institut für Migrationsforschung und Interkulturelle Studien (IMIS).

Marschalck, P., Wiedl, KH (Hrsg.) (2001). Migration und Krankheit (= IMIS-Schriften 10), Osnabrück: Universitätsverlag Rasch

Marsick, V. J., Watkins, K. E. (1990). Informal and incidental learning in the workplace. New York: Routledge.

Mattes, M. (2005). „Gastarbeiterinnen“ in der Bundesrepublik: Anwerbepolitik, Migration und Geschlecht in den 50er bis 70er Jahren. Frankfurt: Campus. 
McEwen, B., Mendelson, S. (Eds.) (1993). Effects of stress on the neurochemistry and morphology of the brain: Counterregulation versus damage (2nd ed.). New York: Free Press.

McGrath, J.E. (1970). Major methodological issues. In J.E McGrath (Ed.), Social and psychological factors in stress (pp. 19 -49). New York: Holt, Rineheart \& Winston.

Meier-Braun, K.-H. (1995, 25. August). 40 Jahre „Gastarbeiter“ und Ausländerpolitik in Deutschland. Aus Politik und Zeitgeschichte, S. 14-22.

Meier-Braun, K.-H. (2002). Deutschland, Einwanderungsland. Frankfurt am Main: Suhrkamp.

Meier-Braun, K.H. zit. nach Franz Hamburger Abschied von der interkulturellen Pädagogik: Plädoyer für einen Wandel. (Kopie: Angaben unbekannt).

Merten, R., (2006). Bildung und soziale Ungleichheiten - Sozialpädagogische Perspektiven auf ein unterbelichtetes Verhältnis. In: Fatke, R., Merkens, H. (Hrsg.), Bildung über die Lebenszeit. Wiesbaden: VS Verlag

Müller-Wille, C. (2001). Zur Lebenssituation älterer Migranten - Lebensbiografische und familiendynamische Aspekte. In: Älter werden in Deutschland. Fachtagung zu einer Informationsreihe für ältere Migranten, Nr. 11 (S. 29). Berlin/Bonn.

Mummendey, A., Waldzus, S. (2004). National differences and European plurality: Discrimination or tolerance between European countries. In Herrmann, R.K., Risse, T., Brewer, M.B. (Eds.), Transnational Identities. Becoming European in the EU (pp. 59 - 72). Plymouth: Lanham Rowman \& Littlefield Publishers, Inc.

Nadig M. (2010): Zur Psychodynamik transkultureller Begegnungen, In: Golsabahi S., Küchenhoff B., Heise T. (Hrsg.): Migration und kulturelle Verflechtungen, 3. Kongress des Dachverbandes der transkulturellen Psychiatrie, Psychotherapie und Psychosomatik im deutschsprachigen Raum e.V., (DTPPP) (S. 59-68). Berlin: VWB.

Neupert, S. D., Almeida, D. M., Charles, S. T. (2007). Age differences in reactivity to daily stressors: The role of personal control. In: Journal of Gerontology, Series B: Psychological and Behavioral Sciences, 62: 216-225.

Nokielski, H. (2005). Transnationale Ruhestandsmigration, in: Thomas Drepper, Andreas Göbel, Hans Nokielski (Hrsg.), Sozialer Wandel und kulturelle Innovation: historische und systematische Perspektiven ; Eckart Pankoke zum 65. Geburtstag, Berlin: Duncker \& Humblot.

Nokielski, H.(2005).Transnationale Ruhestandsmigration. In: Drepper, T., Göbel, A., Nokielski, H. (Hrsg.): Sozialer Wandel und kulturelle Innovation: historische und systematische Perspektiven; Eckart Pankoke zum 65. Geburtstag (S. 311-334), Berlin: Duncker \& Humblot.

Nolda, S. (2008). Einführung in die Theorie der Erwachsenenbildung. Darmstadt: WBG.

Nonaka, I., Takuchi, H. (1997). Die Organisation des Willens. Frankfurt /New Yok: Campus Verlag.

O`Reilly, K., Benson, M. (2009). Lifestyle Migration: Escaping to the Good Life? (Tippels 2206: 11 http://www.ashgate.com/pdf/SamplePages/Lifestyle_Migration_Ch1.pdf , Zugriff: Januar 2011. 
Oberndörfer, D. (2001). Dialog der Kulturen oder Parallelgesellschaft - Funktionen und Wirkungen fremdsprachlicher Medienangebote. In: Ausländerbeauftragte der Freien und Hansestadt Hamburg und der HAM (Hrsg.), Medien Migration Integration. Elektronische Massenmedien und die Grenzen kultureller Identität (S.15-25). Berlin: Hamburgische Anstalt für neue Medien.

O'Reilly, K. und Benson, MC.(2009). Lifestyle migration: escaping to the good lie. In Benson, M., O'Reilly, K. (Hrsg.), Lifestyle migration: expectations, aspirations and experiences (pp. 1-14). Farnham: Ashgate.

Özdamar, E. S. (1991) Mutterzunge. Berlin: Rotbuch.

Özkan, I. (2009). Ressourcenorientierte Stabilisierung in der traumazentrierten Behandlung von Migranten. In: Solmaz, G., Stompe, T., Heise, T. (Hg.). Jeder ist weltweit ein Fremder. 2. Kongress des DTPPP in Wien 2008 (S. 207 - 215). Das transkulturelle Psychoforum Bd. 16. Berlin: VWB-Verlag.

Özkan, I. (2011) (IN DRUCK). Transkulturelle Psychotherapie: state of the art und das Besondere daran. In: Heise, T. (2011). Mit Leib und Seele ankommen - Psychische und psychosomatische Krankheiten in unserer Zuwanderungsgesellschaft. 4. Kongress des DTPPP in Düsseldorf 2010. Berlin: VWB Verlag.

Panksepp, J. (Hrsg.) (2004). Textbook of Biological Psychiatry. Hoboken: Wiley-Liss

Panksepp, J. , Moskal, J.R. , Panksepp, J.B., Kroes, R.A. (2002) Comaparative Approaches in Evolutionary Psychology: Molecular Neuroscience Meets the Mind. In: Neuroendocrinology Letters Special Issue Vol. 23: $113 \mathrm{f}$.

Pavkovic, Gari (2001). Sicht- und Erlebnisweisen sowie Bewältigungsstile von Krankheit in verschiedenen Kulturen. In: Migrationsspezifische Begutachtung im Spannungsfeld von Medizin, Recht Psychologie und Politik. Fachtagung Hannover, 2./3. Nov. 2001, S. 37.

Pazarkaya, Y. (1989). Rosen im Frost: Einblicke in die türkische Kultur. Zürich: Unionsverlag.

Phinney, J., Horenczyk, G., Liebkind, K., Vedder, P. (2001). Ethnic identity, immigration, and well-being: An interactional perspective. Journal of Social Issues, 57: 493-510.

Pietät Ernst Bestattungen GbR. (2009). http://www.bestattungenernst.de/cid/index.html?dlr-Page_uni_008/index.html, Zugriff am 15.09.2009

Platon (1982). Der Staat (Originaltitel: Politeia). Ditzingen: Reclam.

Raufelder, D., Mohr, S. (2010). Zur Bedeutung sozio-emotionaler Faktoren im Kontext Schule unter Berücksichtigung neurowissenschaftlicher Aspekte. In: Ittel, A. et al. (Hg.). Jahrbuch Jugendforschung. Wiesbaden: VS.

Redfield R., Linton R., Herskovits, M.J. (1936). Memorandum for the Study of Acculturation. American Anthropologist, Vol. 38, No. 1: 149-152.

Regal, W., Nanut, M. (2005). Vom Seeräuberbein bis zur Kunsthand (Narrentum 20). In: Ärzte Woche 27/2005. Wien: Springer.

Rodewig, K. (2002). Psychosomatische Rehabilitation türkischstämmiger Migranten. In: Dettmers, C., Albrecht, N-J., Weiller,C. (Hrsg.), Gesundheit, Migration, Krankheit, Sozialmedizinische Probleme und Aufgaben in der Nervenheilkunde (S. 204-217). Bad Honnef: Hippocampus. 
Rodewig, K. (2002). Psychosomatische Rehabilitation türkischstämmiger Migranten. In C. Dettmers, N-J. Albrecht und C. Weiller (Hrsg.), Gesundheit, Migration, Krankheit, Sozialmedizinische Probleme und Aufgaben in der Nervenheilkunde (S. 204-217). Bad Honnef: Hippocampus

Rosenthal, G. (2002). Transgenerationale Folgen von Verfolgung und Täterschaft. Familien von Shoah-Überlebenden und von Nazi-Tätern. In: StreeckFischer/Sachsse/Özkan (Hg.), Körper - Seele - Trauma. Biologie, Klinik und Praxis (S. 174-2017). Göttingen: Vandenhoeck und Ruprecht.

Ruhs, D. (2008). Duale Identität: Konsequenzen und Antezedenzien im Kontext gesellschaftlicher Partizipation. Hamburg: Kovač.

Rustemeyer, R. (2007). Einführung in die Unterrichtspsychologie (2. Aufl.). Darmstadt: WBG.

Salman, R. (1995). Hintergründe gelungener Migration. In: Koch E, Özek, M., Pfeiffer, W.M., (Hg.) Psychologie und Pathologie der Migration (S. 90-100). Freiburg im Breisgau: Lambertus- Verlag.

Santel, B., Weber, A. (2000). Migrations- und Ausländerrecht in Deutschland. In: Bade, K.J., Münz, R. (Hrsg.), Migrationsreport 2000 Fakten - Analysen - Perspektiven (S. 109-140). Für den Rat für Migration herausgegeben von Klaus Bade und Rainer Münz. Bundeszentrale für politische Bildung. Bonn.

Sapolsky, R.M. (1994). Warum Zebras keine Migräne kriegen. Wie Streß den Menschen krank macht. Aus dem Amerikanischen von Stein, B. München Zürich: Piper

Schaub, H., Zenke, K. G. (2000). Wörterbuch Pädagogik. München: Deutscher Taschenbuch Verlag.

Schimany, P., Schock, H. (2006). Migration und ethnische Minderheiten. http://www.gesis.org/sowiport/fileadmin/user_upload/soFid/Migration_und_ethnische _Minderheiten/Migration_2006_01.pdf , aufgerufen: Januar 2011.

Schirrmacher, C. (2006). Ist Multi-Kulti am Ende? Keine Alternative zur Wertediskussion. Vortrag im Festsaal des Roten Rathauses, Berlin-Mitte am 15.2.2006. Evangelische Verantwortung, März 2006: 6-15.

Schmeling-Kludas, C., Boll-Klatt, A., Fröschlin, R. (2002). Was lässt türkische Migranten psychosomatisch erkranken? - Rückschlüsse aus einer retrospektiven Aktenanalyse. In: Dettmers, Ch., Albrecht, N.J., Weiller, C. (Hrsg.), Gesundheit, Migration und Krankheit. Sozialmedizinische Probleme und Aufgaben in der Nervenheilkunde (S. 195-203). Bad Honnef: Hippocampus Verlag.

Schulz, M., Stamov- Roßnagel, C. (2010). Informal workplace learning: An exploration of age differences in learning competence. Learning and Instruction, 20: 383-399.

Seeberger, B. (2001). Forschungsarbeiten im Forschungsschwerpunkt: Alter und Islam. (Innerethnische) Hilfs- und Unterstützungssysteme für ältere türkische Migranten im Großraum Nürnberg. Dissertation 2001. Internetquelle: http://www.berndseeberger.de/Alter_und_Islam.htm, Zugriff Januar 2003

Seligman, M. E. P. (1975). Helplessness. On Depression, Development and Death. San Francisco: Freeman and Comp.

Selye, H. (1936). A Syndrome produced by diverse nocuous agents. Nature 138: 32.

Selye H. (1946). The general adaptation syndrome and the diseases of adaptation. Journal of Clinical Endocrinology 6:117-231. 
Selye, H. (1950). The Physiology and Pathology of Exposure to Stress, ACTA. INC. Medical Publishers.

Şen, F. (1998). Die Situation der türkischen Migranten heute. In: Eryılmaz, A., Jamin, M. (Hrsg.), Fremde Heimat (S. 369-390). Essen: Klartext Verlag.

Sendera, A., Sendera M. (2007). Skills-Training bei Borderline- und Posttraumatischer Belastungsstörung. Wien: Springer.

Seneca, epistulae morales ad Lucilium 106, 12.

Shors, TJ. (2006). Stressful experience and learning across the lifespan. In: Annual Review of Psychology. 57: 55-85.

Simpsons, The (1995), Sweet Seymour Skinners Baadasssss Song. Folge 19 Staffel 5, Erstausstrahlungsdatum laut imdb.com 17. Juni 1995, Produktionscode 1F18, http://www.imdb.com/title/tt0777150/; http://www.snpp.com/episodes/1F18.html, letzter Zugriff: 15.12.2010.

Skolimowska J, Wesierska M, Lewandowska M, Szymaszek A, Szelag E. (2011). Divergent effects of age on performance in spatial associative learning and real idiothetic memory in humans. In: Behavioural Brain Research vol.218 no.1: 87-93.

Sluzki, C.E. (2001). Psychologische Phasen der Migration und ihre Auswirkungen. In: Hegemann Th., Salman, R. (Hrsg.), Transkulturelle Psychiatrie, Konzepte für die Arbeit mit Menschen aus anderen Kulturen (S. 101-129). Bonn: Psychiatrie Verlag.

Sonnenberger, K. (2003). Verwaltete Arbeitskraft: die Anwerbung von „Gastarbeiterinnen“ und "Gastarbeitern" durch die Arbeitsverwaltung in den 9150er und 1960 Jahren. In: Oltmer, J. (Hrsg.), Migration steuern und verwalten (S.145-176). Göttingen: V\&R unipress.

Spiegelonline (2006). Der Abstieg der Rütli-Schule.

http://www.spiegel.de/politik/deutschland/0,1518,409011,00.html . Letzter Zugriff: 01.08.2010.

Spiel, C. (2006). Grundkompetenzen für lebenslanges Lernen - eine Herausforderung für Schule und Hochschule? In: Fatke, R., Merkens, H. (Hrsg.), Bildung über die Lebenszeit (S. 85-97). Wiesbaden: VS Verlag.

Statistisches Bundesamt (2009a). Bevölkerung und Erwerbstätigkeit. Ausländische Bevölkerung. Ergebnisse des Ausländerzentralregisters. Fachserie 1 Reihe 2 (Ausgabe vom 24. März 2009). Wiesbaden: Statistisches Bundesamt.

Steinert, J. D. (1995). Migration und Politik: Westdeutschland - Europa - Übersee 19451961. Osnabrück: secolo.

Stock, A. (2007). Vom Musterkoffer bis zum Corporate Design. Wiedereröffnung des Werkbundarchivs Berlin. Entnommen von: http://www.dradio.de/dkultur/sendungen/fazit/641411/. Zugriff am 04.08.2009.

Strübing, J. (2008). Grounded Theory. Zur sozialtheoretischen und epistemologischen Fundierung des Verfahren der empirisch begründeten Theoriebildung. Wiesbaden: VS Verlag für Sozialwissenschaften.

Tacke-Borchert, E. (2001). Begutachtung älterer Migranten mit Arbeitsunfähigkeit beim MDK. In: Migrationsspezifische Begutachtung im Spannungsfeld von Medizin, Recht Psychologie und Politik. (S. 41ff). Fachtagung Hannover, 2./3. Nov. 2001. 
Tajfel, H., Turner, J. C. (1979). An Integrative Theory of Intergroup Conflict. In Austin, W.G., Worchel, S. (Hrsg.), The Social Psychology of Intergroup Relations (p. 33-47). Monterey: Brooks-Cole.

Tajfel, H. (1982). Gruppenkonflikt und Vorurteil. Entstehung und Funktion sozialer Stereotypen. Bern: Hans Huber.

Tepecik, A. (2009). Educational Success and intergenerational Transmission: Educational Advancement among Migrants of Turkish Descent in Germany. In: Bogner, A. und Rosenthal, G. (Hrsg.), Ethnicity, Belonging and Biography (p. 371-387). Berlin/Münster: Lit-Verlag.

Thomas, A. (Hrsg.) (1993b). Kulturvergleichende Psychologie: Eine Einführung. Göttingen: Hogrefe.

Tippelt, R., Kasten, C., Dobischat, R., Federighi, P., Feller, A. (2006). Regionale Netzwerke zur Förderung lebenslangen Lernens - Lernende Regionen. In: Fatke, R., Merkens, H. (Hrsg.), Bildung über die Lebenszeit (S. 279-290). Wiesbaden: VS Verlag für Sozialwissenschaften.

Treibel, A. (1999): Migration in modernen Gesellschaften - Soziale Folgen von Einwanderung, Gastarbeit und Flucht. Weinheim; München, 2. Auflage

Trippmacher, B. (1997)( Vorwort. In: Arbeiterwohlfahrt - Abschlußbericht zum Projekt „Aufbau einer Altentagesstätte für türkische Seniorinnen und Senioren“. Oktober 1993 bis Oktober 1996, S. 1. Ort und Verlag unbekannt.

Trouillet R, Gana K, Lourel M, Fort I. (2009). Predictive value of age for coping: the role of self-efficacy, social support satisfaction and perceived stress. In: Aging and Mental Health, 13(3), May 2009: 357-366.

Tsiakalos, G. (1983). Ausländerfeindlichkeit. Tatsachen und Erklärungsversuche. München: Beck.

Tufan, I. (1999). Über die gesundheitliche Lage der älteren türkischen MigrantInnen in Deutschland In: iza: Zeitschrift für Migration und soziale Arbeit (2):50-53.

Turner, J. C., Oakes, P. J., Haslam, S. A., McGarty, C. (1994). Self and collective: Cognition and social context. Personality and Social Psychology Bulletin, 20: 454463.

Turner, J.C., Hogg, M.A., Oakes, P.J., Reicher, S., Wetherell, M.S. (1987). Rediscovering the social group: A self-categorization theory. Oxford: Basil Blackwell.

van Dijk, R., Wesenbeek, R. (Jahr unbek.). Zehn Handlungspunkte für eine interkulturelle geistige Gesundheitsfürsorge. In: IGCZ Niederland

Vedder, P., Sam, D. L., Liebkind, K. (2007). Adaptation of adolescents with Turkish background in Europe. Applied Developmental Science, 11: 126-136.

Verkuyten, M. (2005). Ethnic group identification, and group evaluations among minority and majority groups: Testing the multiculturalism hypothesis. Journal of Personality and Social Psychology, 88: 121-138.

Verkuyten, M., und Yildiz, A.A. (2007). National (dis)identification, and ethnic and religious identity: A study among Turkish-Dutch Muslims. Personality and Social Psychology Bulletin, 33: 1448-1462.

von Lersner, Ulrike (2011). Bicultural Identity Identification and Mental Health in Adolescent Migrants. European Journal of Psychotraumatology Supplement 1, 2011: 47. 
von Lersner, Ulrike, Vortragsfolie. 12th European Conference on Traumatic Stress, Wien, 05.06.2011

von Wilamowitz-Moellendorff, U. (2001). Türken in Deutschland - Einstellungen zu Staat und Gesellschaft. Arbeitspapier herausgegeben von der Konrad-Adenauer-Stiftung e.V..

von Wilamowitz-Moellendorff, U. (2002). Türken in Deutschland II - Individuelle Perspektiven und Problemlagen. Arbeitspapier herausgegeben von der KonradAdenauer-Stiftung e.V..

Wagner, C. (2001). Paulo Freire (1921 - 1997). Alphabetisierung als Erziehung zur Befreiung. Entwicklung und Zusammenarbeit, 1 (2001): 17-19.

Waller, E., Scheidt, C.E. (2002). Somatoforme Störungen und Bindungstheorie. Psychotherapeut, 47:157-164.

Weaver, G. R. (1998). Understanding and Coping with Cross-cultural Adjustment Stress. In Weaver, G.R. (ed.), Culture, Communication and Conflict: Readings in Intercultural Relations, second edition (pp. 169-189). Needham Heights: Simon \& Schuster Publishing.

Weinold, M. (2010). Spezialprobleme des Übersetzens SP-- - DE/DE-- - SP; Das Anredesystem des Deutschen. http://www.carstensinner.de/Lehre/spanisch/dossiers/Duzen_und_Siezen_im_Deuts chen.pdf, 04.01.2011

Westle, B. (1999). Kollektive Identität im vereinten Deutschland. Nation und Demokratie in der Wahrnehmung der Deutschen. Opladen. Leske \& Budrich.

Wollenschläger, M. (1994). Migrationspolitik und Zuwanderungsrecht, Staatsbürgerschaft und Integration. In: Bade, K.J. (Hrsg.), Das Manifest der 60: Deutschland und die Einwanderung. München: Beck.

Wong P., Wong L. (Hrsg) (2006). Handbook of Multicultural Perspectives on Stress and Coping. New York: Springer.

Wong, R., Gonzalez-Gonzalez, C. (2010). Old-Age Disability and Wealth Among Return Mexican Migrants From the United States. In: Journal of Aging and Health 22 (7): 932-954.

Wulf, C., Zirfaß, J. (2006). Bildung als performativer Prozess - ein neuer Fokus erziehungswissenschaftlicher Forschung. In: Fatke, R., Merkens, H. (Hrsg.), Bildung über die Lebenszeit (S. 291-301). Wiesbaden: VS Verlag für Sozialwissenschaften.

Yano, H. (1998). „Wir sind benötigt, aber nicht erwünscht.“. In: Eryılmaz, A., Jamin, M. (Hrsg.), Fremde Heimat (S. 39-61). Essen: Klartext Verlag.

Yeaxlee, B. A. (1929). Lifelong education. A sketch of the range and significance of the adult education movement. London: Cassell.

Yilmaz, T. (2001) Pflegeversicherungsberatung mit türkischen Seniorinnen und Senioren. Erfolge trotz schwierigem Zugang. Erfahrungen im Seniorenbüro International Essen. In: Kaewnetara, E., Uske, H. (Hrsg.), Migration und Alter. Auf dem Weg zu einer kulturkompetenten Altenarbeit. Konzepte - Methoden - Erfahrungen (S. 1123). Duisburg: Unrast-Verlag.

Zagefka, H., Brown, R. (2002). The relationship between acculturation strategies, relative fit and intergroup relations: immigrant-majority relations in Germany. European Journal of Social Psychology, 32: 171-188. 
Zick, A. (2010). Psychologie der Akkulturation: Neufassung eines Forschungsbereiches. Wiesbaden: VS Verlag.

Zürcher, R. (2007). Informelles Lernen und der Erwerb von Kompetenzen. Theoretische, didaktische und politische Aspekte. Materialien zur Erwachsenenbildung Nr. 2/2007, Herausgegeben von Bundesministerium für Unterricht, Kunst und Kultur, Abteilung Erwachsenenbildung V/8, Wien. 
Erklärung

Erklärung gem. „§ 18 Versicherung“ der Promotionsordnung Promotionsordnung der Sozialwissenschaftlichen Fakultät der Georg-August-Universität Göttingen vom 17.10.2005 mit Änderungen vom 12.02.2007:

Ich versichere, dass ich die eingereichte Dissertation:

„Migrationsstress, Alter und Lernen -

Betrachtungen der Zusammenhänge sowie Implikationen für die Didaktik (am Beispiel in Deutschland lebender, gealterter türkischer Menschen)“

selbständig und ohne unerlaubte Hilfsmittel verfasst habe. 2Anderer als der von mir angegebenen Hilfsmittel und Schriften habe ich mich nicht bedient. 3Alle wörtlich oder sinngemäß den Schriften anderer Autoren entnommenen Stellen habe ich kenntlich gemacht.

Ibrahim Özkan 